\title{
Development and Application of an Analysis Methodology for Interpreting Ambiguous Historical Pressure Data in the WIPP Gas-Generation Experiments
}

prepared by

Engineering Technology Division

Argonne National Laboratory-West 


\section{About Argonne National Laboratory}

Argonne is managed by The University of Chicago for the U.S. Department of Energy under contract W-31-109-Eng-38. The Laboratory's main facility is outside Chicago, at 9700 South Cass Avenue, Argonne, Illinois 60439. For information about Argonne and its pioneering science and technology programs, see www.anl.gov.

\section{Availability of This Report}

This report is available, at no cost, at http://www.osti.gov/bridge. It is also available on paper to U.S. Department of Energy and its contractors, for a processing fee, from:

U.S. Department of Energy

Office of Scientific and Technical Information

P.O. Box 62

Oak Ridge, TN 37831-0062

phone (865) 576-8401

fax (865) 576-5728

reports@adonis.osti.gov

\section{Disclaimer}

This report was prepared as an account of work sponsored by an agency of the United States Government. Neither the United States Government nor any agency thereof, nor The University of Chicago, nor any of their employees or officers, makes any warranty, express or implied, or assumes any legal liability or responsibility for the accuracy, completeness, or usefulness of any information, apparatus, product, or process disclosed, or represents that its use would not infringe privately owned rights. Reference herein to any specific commercial product, process, or service by trade name, trademark, manufacturer, or otherwise, does not necessarily constitute or imply its endorsement, recommendation, or favoring by the United States Government or any agency thereof. The views and opinions of document authors expressed herein do not necessarily state or reflect those of the United States Government or any agency thereof, Argonne National Laboratory, or The University of Chicago. 


\section{Development and Application of an Analysis Methodology for Interpreting Ambiguous Historical Pressure Data in the WIPP Gas-Generation Experiments}

by

F.S.Felicione*

Engineering Technology Division, Argonne National Laboratory-West

*Current Affiliation: Idaho National Laboratory, P.0.Box 1625, Idaho Falls, ID 83415
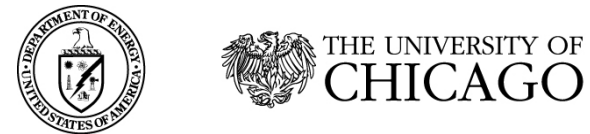


\section{TABLE OF CONTENTS}

Page

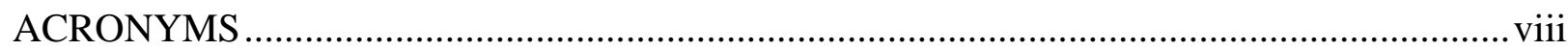

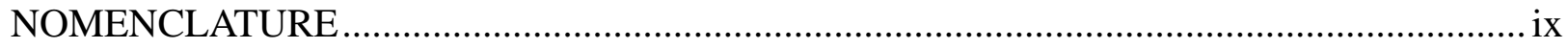

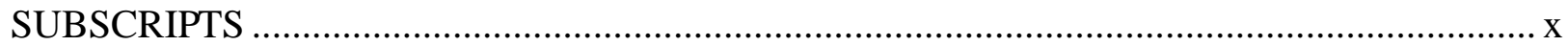

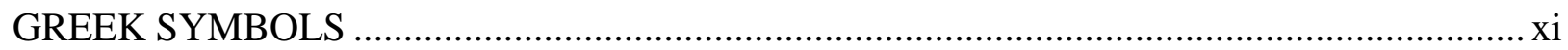

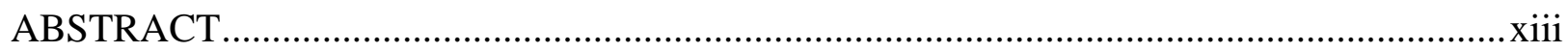

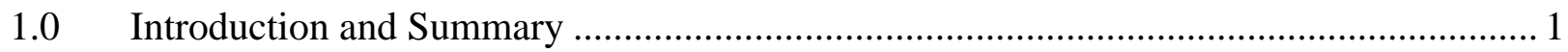

$1.1 \quad$ Gas-Generation Experiments and Task Background ............................................ 1

1.2 Operational Experience with the GGE ……….............................................. 3

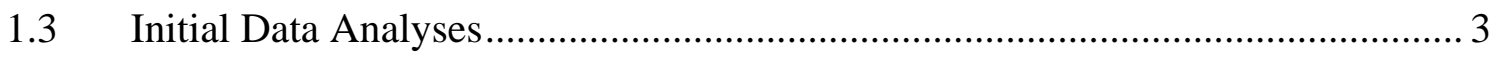

$1.4 \quad$ Approach and Objectives of the Present Investigation ............................................ 5

1.4.1 Validation of the Test Data........................................................................ 5

1.4.2 Recovery of the Archived Pressure Data ..................................................... 6

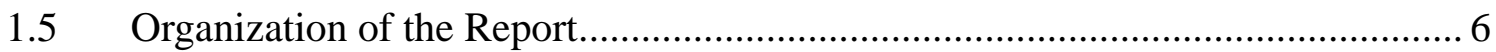

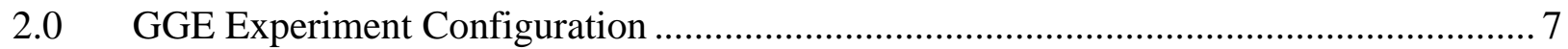

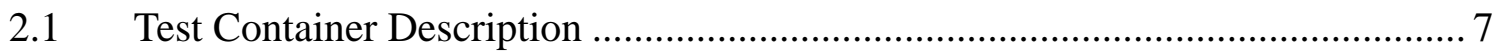

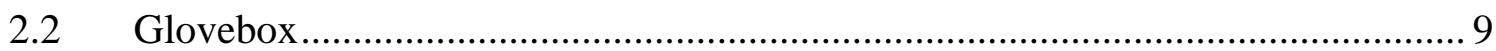

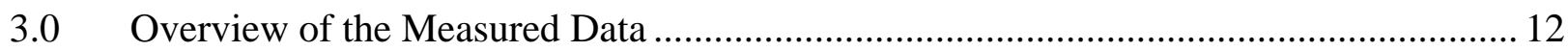

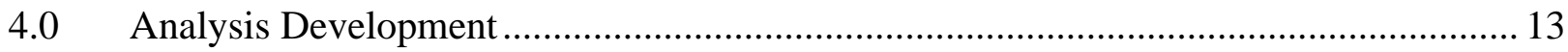

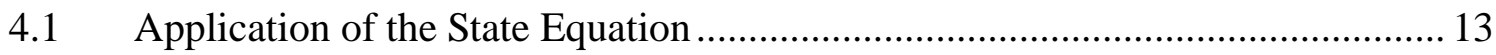

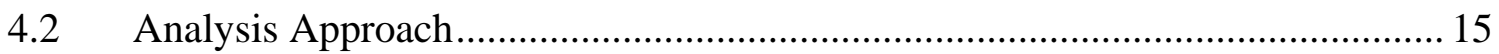

5.0 Headspace-Gas-Mass Considerations ............................................................................ 15

$5.1 \quad$ Headspace-Gas Composition and Volatility ……………………....................... 15

5.2 Sources of Variation in the Headspace-Gas Mass ................................................ 17

5.2.1 Discrete Headspace-Gas-Mass Variation.................................................... 17

5.2.2 Continuous Headspace-Gas-Mass Variation............................................... 19

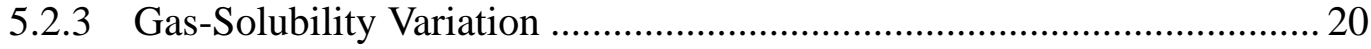

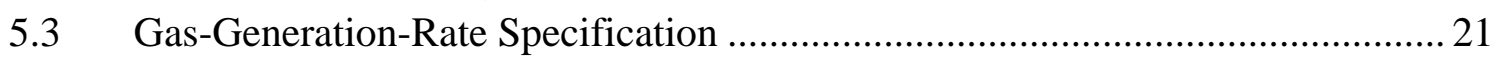

5.3.1 Increase in Headspace-Gas Mass by Gas Generation.................................. 21

5.3.2 Mole Fraction of Generated Gases …………………………………...... 23

5.3.3 Rates of Gas Generation ..................................................................... 23

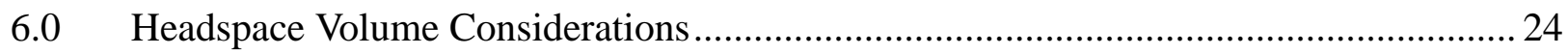

$6.1 \quad$ Headspace Volume Variation ............................................................................ 24

6.1.1 Test-Container Expansion/Contraction .................................................... 25

6.1.2 Expansion/Contraction in the Brine and Waste ......................................... 26

$6.2 \quad$ Headspace Volume Determination................................................................... 26

6.2.1 Headspace-Gas Expansion Measurements .............................................. 27 


\section{TABLE OF CONTENTS (Contd.)}

6.2.2 Adjustment of $\underline{\text { Page }}$

6.2.2 Adjustment of the Headspace Volume Measurements........................... 29

7.0 Headspace-Gas Compressibility ............................................................................. 31

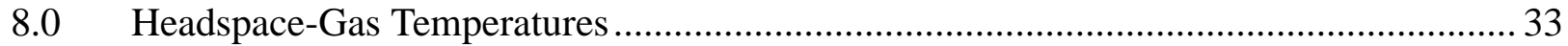

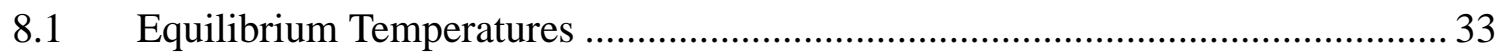

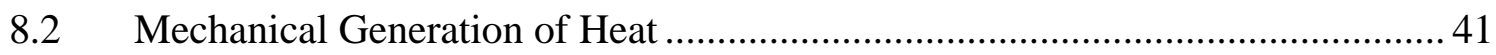

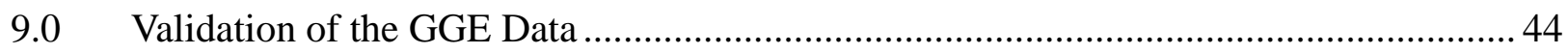

9.1 Computation at a Specific Point ………………............................................. 44

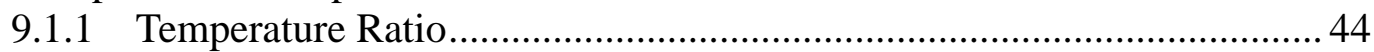

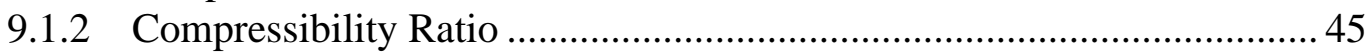

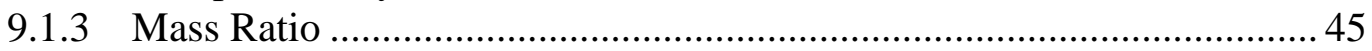

9.1.4 Headspace-Volume Ratio....................................................................... 46

9.1.5 Predicted Pressure ................................................................................... 48

9.1.6 Compensated Pressure ………………………………………………... 49

9.1.7 Mechanical Work ................................................................................... 49

9.2 Adjustment of the Pressures in the Blank, Experiment-Control

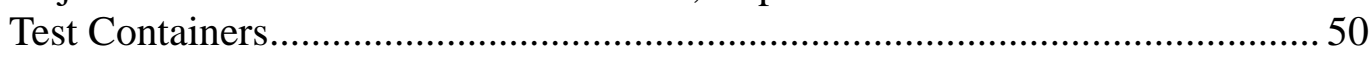

10. Recovery of the Archived Pressure Measurements .........................................................5 55

10.1 Determination of the Brine/Waste Thermal Expansion Coefficient ..................... 55

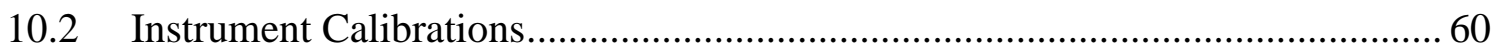

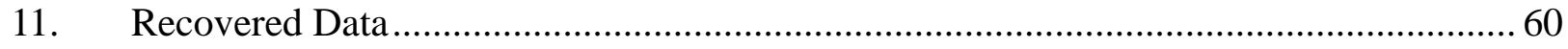

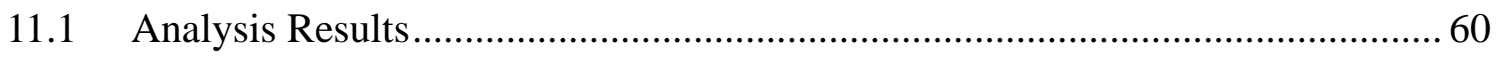

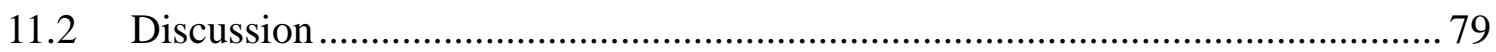

11.2.1 Adjusted Pressure Results......................................................................... 79

11.2.2 Examination for Evidence of Gas Generation ........................................... 82

12.0 Applicability to Other Investigations and Experiment-Design Implications..................... 84

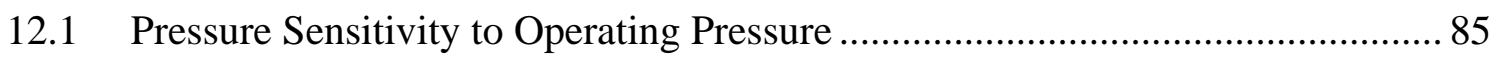

12.2 Pressure Sensitivity to Headspace/Waste Volume Ratio......................................... 85

12.3 Comparison of the GGE to Other Gas-Generation Experiments........................... 85

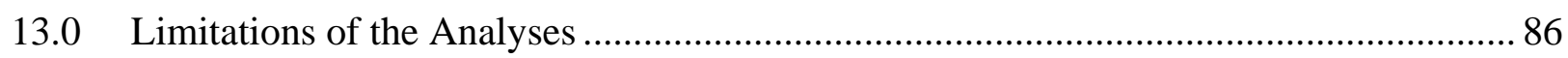

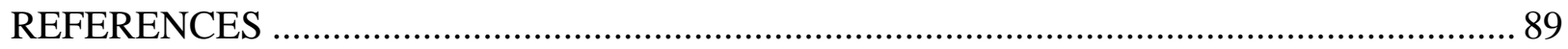

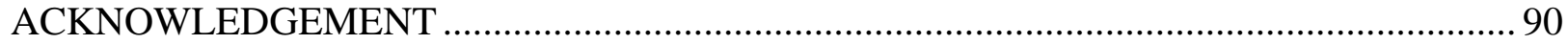

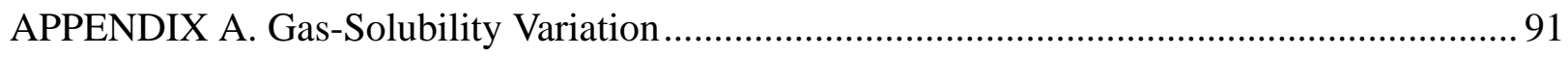

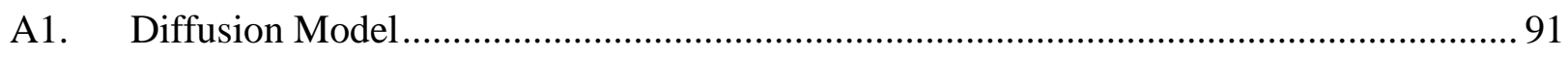

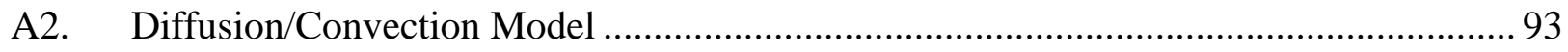

APPENDIX B. Thermal Expansion Experiments …………………………………………........ 99 


\section{TABLE OF CONTENTS (Contd.)}

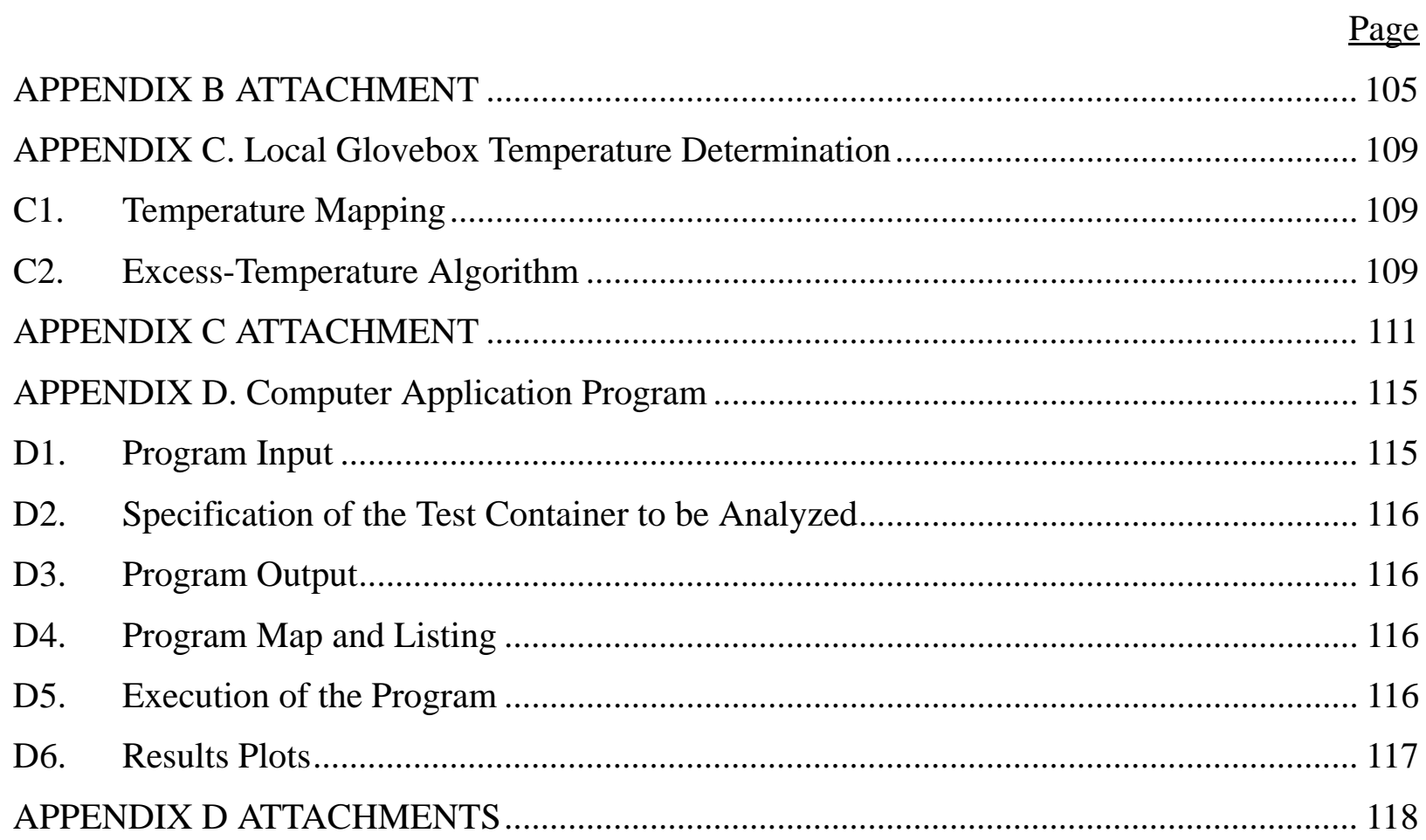




\section{LIST OF FIGURES}

Page

1. Temperature Trace for a Step Increase in the Heater Setpoint ................................................ 4

2. Pressure Trace for a Step Increase in the Heater Setpoint .................................................... 5

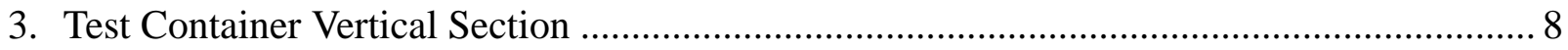

4. Test-Container Shroud and Heater-Band Arrangement......................................................... 9

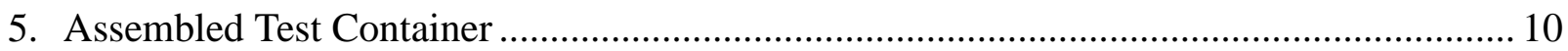

6. Assembled Test Container in Glovebox with Instrumentation Connections......................... 11

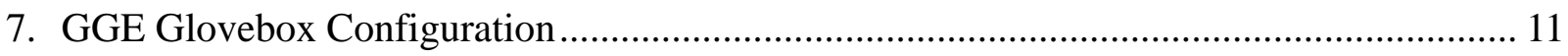

8. Test Container and Glovebox Temperatures for a Typical Test Container ................................ 12

9. Synchronization of Test Container Pressure with Temperature.............................................. 13

10. Representation of Discrete Events at Times Prior To and Beyond the

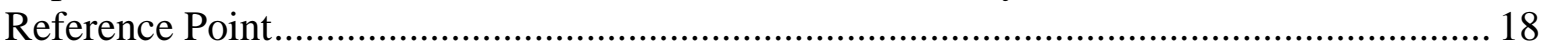

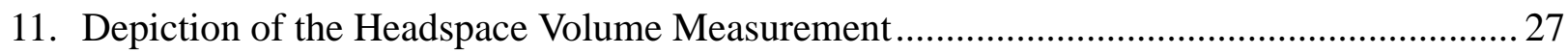

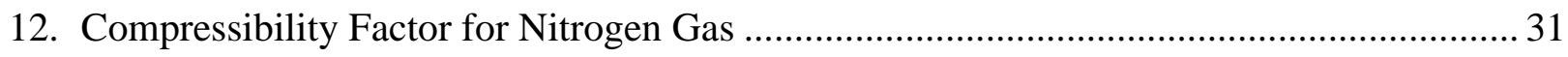

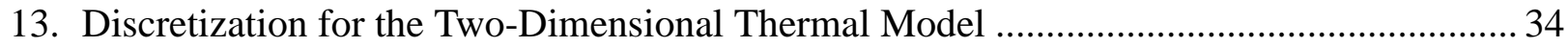

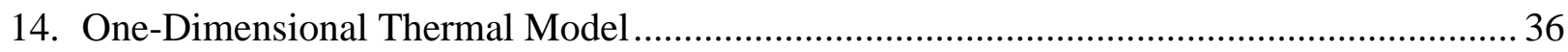

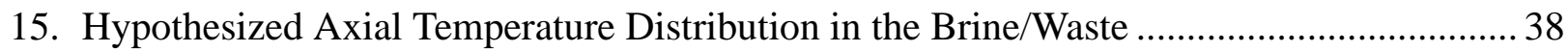

16. Two-Dimensional Effects in the Test-Container Head Region ................................................ 38

17. Mechanical Work of Compression in the Headspace Gas ...................................................... 42

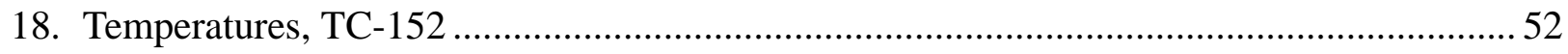

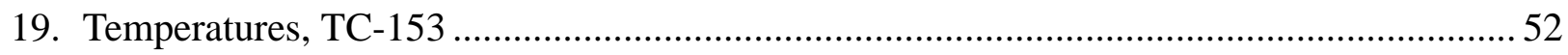

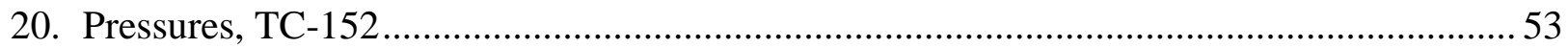

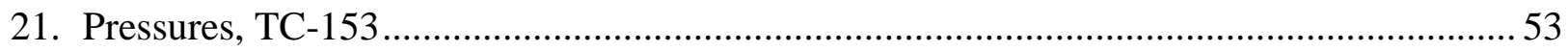

22. Gas Generation in TC-152 ................................................................................... 54

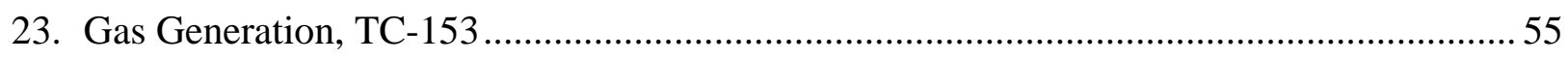

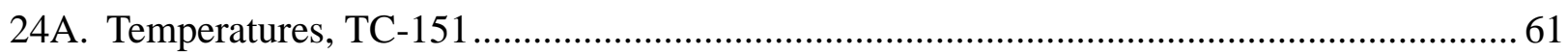

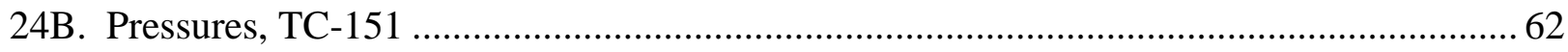

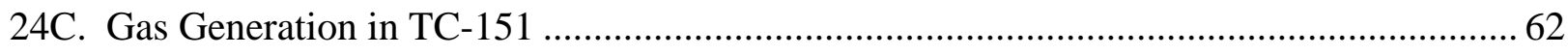

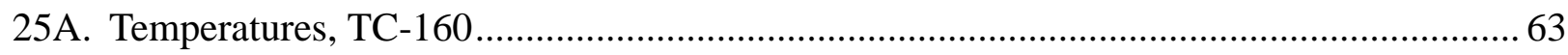

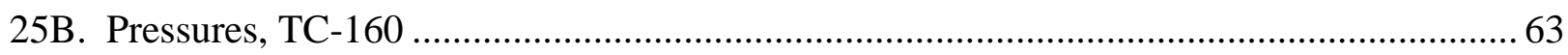

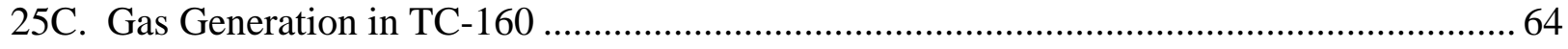

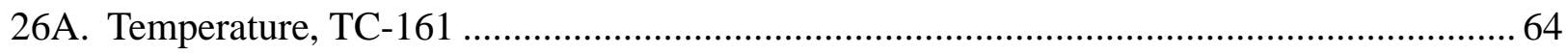

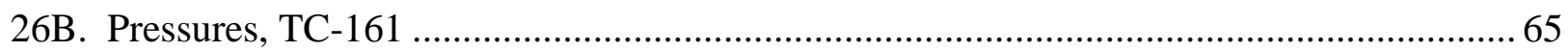

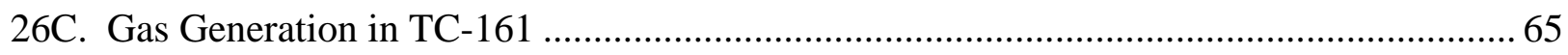

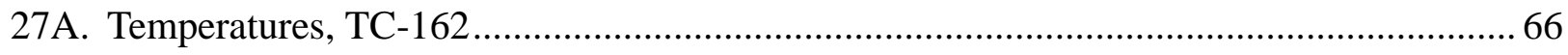

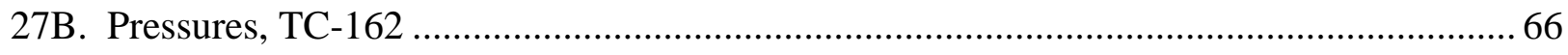

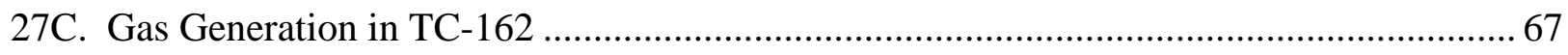

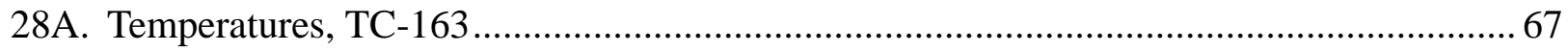

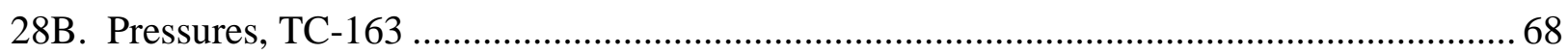




\section{LIST OF FIGURES (Contd.)}

Page

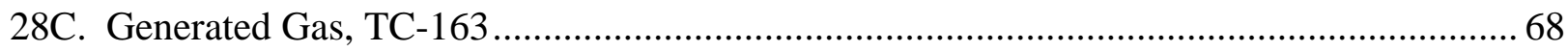

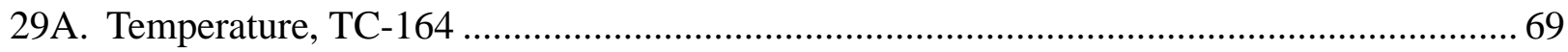

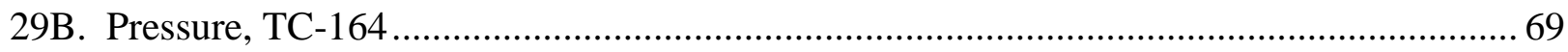

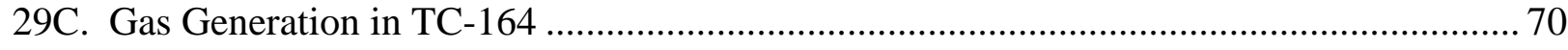

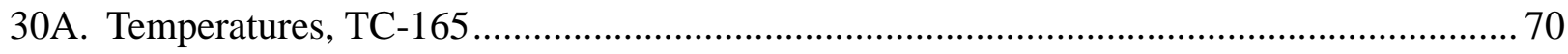

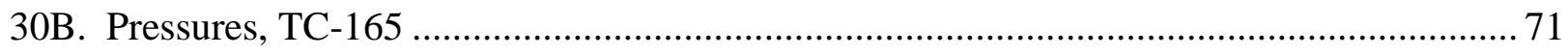

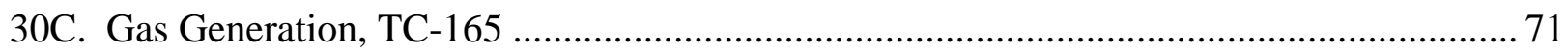

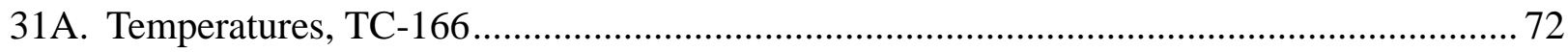

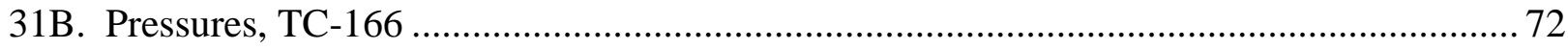

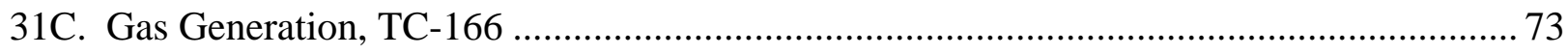

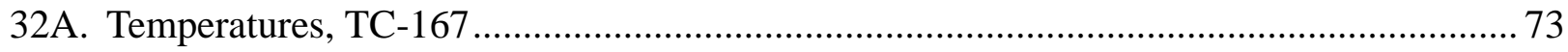

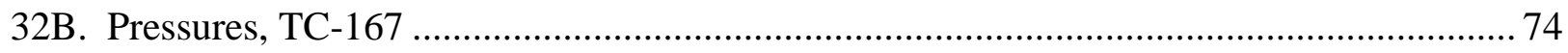

32C. Gas Generation, TC-167 ………………………..................................................... 74

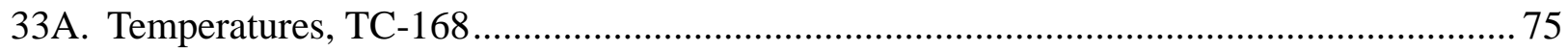

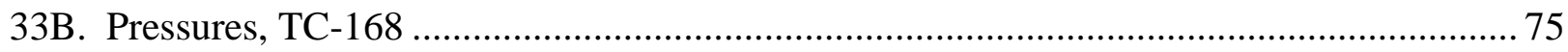

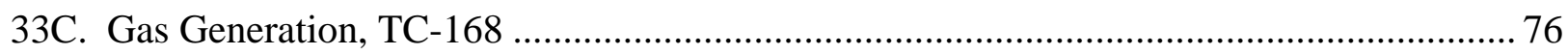

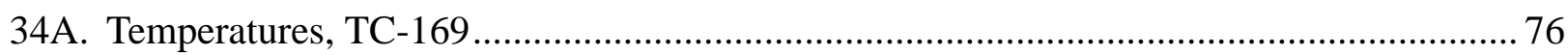

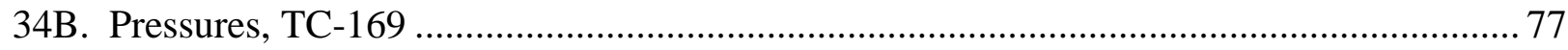

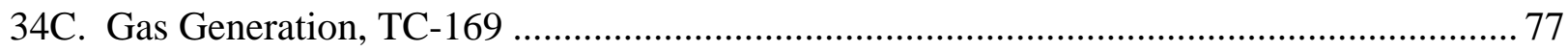

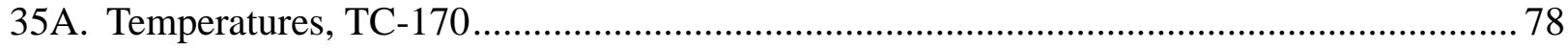

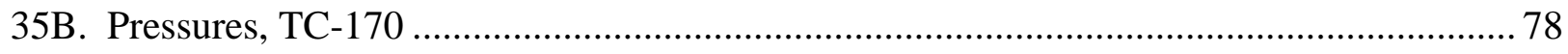

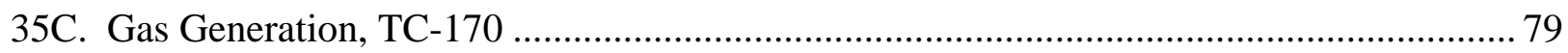

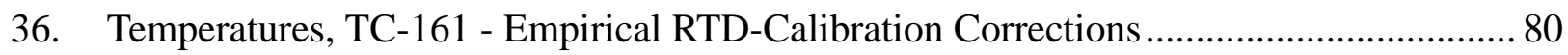

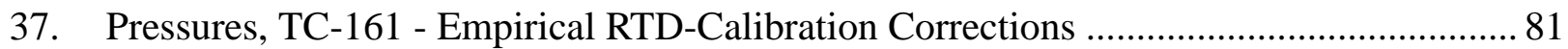

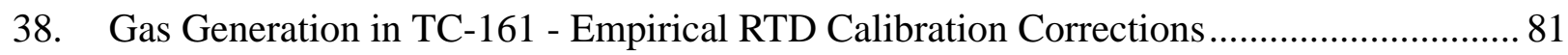

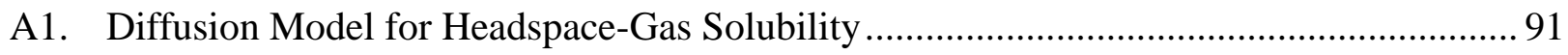

A2. Solution to the Pure Diffusion Model ................................................................................. 93

A3. Heat-Conduction and Convection Model for Determining the Temperature ...................... 94

A4. Enhancement to the Thermal Conductivity to Account for Natural Convection................ 95

A5. Diffusion/Convection Model for Gas Solubility in the Brine ............................................. 96

B1. Experiment Configuration for Determination of the Volumetric, Thermal-Expansion Coefficients for Pure Water and Brine.................................................. 99

B2. Experiment Apparatus for Determination of the Volumetric,

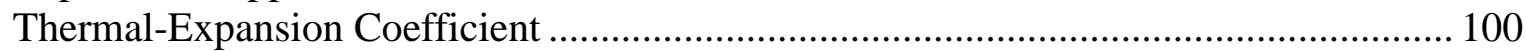

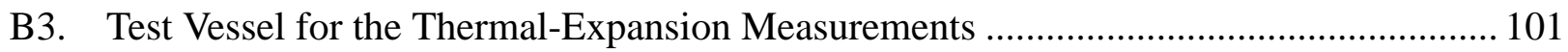

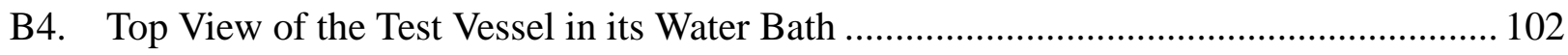

B5. Measured Volumetric Coefficients of Thermal-Expansion for Pure Water ....................... 103

B6. Measured Volumetric Coefficients of Thermal-Expansion for Brine A ........................... 103

C1. Estimate of Local Test Container Temperature Excess...................................................... 110 


\section{LIST OF TABLES}

$\underline{\text { Page }}$

1. Waste Contents Within Each Test Container ................................................................... 1

2. Best Estimate Headspace Volumes at the Reference Conditions .................................... 30

3. Thermo-Physical Properties Used in the Thermal Model ............................................... 40

4. Raw Headspace Volume Measurements for TC-153 .................................................... 46

5. Adjusted Headspace Volume Measurements for TC-153 ............................................... 47

6. Volumetric Coefficients of Thermal Expansion in the Brine/Waste................................... 59

7. Comparison of RTD Calibration Corrections for TC-161: Recorded versus

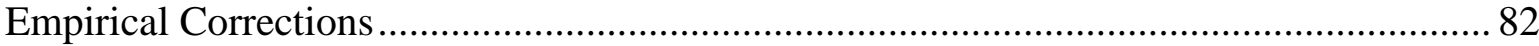

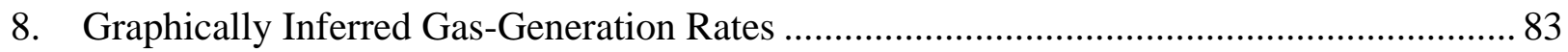




\section{ACRONYMS}

AISI American Iron and Steel Institute

ANL-West Argonne National Laboratory - West

DOE [U.S.] Department of Energy

GGE Gas-Generation Experiments

ID Internal diameter

PID Proportional, integral, and derivitive [controller]

PXD Pressure transducer

RTD Resistance temperature detector

SNL Sandia National Laboratory

TRU Transuranic

UNS Unified Number System

WIPP Waste Isolation Pilot Plant

ZPPR Zero Power Physics Reactor 


\section{NOMENCLATURE}

A Constant defined by Equations 7-2 and 7-3

a $\quad$ Constant defined by Equations 7-2 and 7-3

B Constant defined by Equations 7-2 and 7-3

b Constant defined by Equations 7-2 and 7-3

c Constant defined by Equations 7-2 and 7-3

$\mathrm{c}_{\mathrm{v}} \quad$ Specific heat capacity at constant volume

e $\quad$ Constant defined by Equations 7-2 and 7-3

h Heat-transfer coefficient or conductance value

[MW] Molecular weight

M Mass

N Moles

P Pressure

Q Heat transfer to the headspace gas

q Heat flux

R Gas constant (mass specific)

$\mathrm{R}_{\mathrm{U}} \quad$ Gas constant (mole specific)

s Entropy

T Temperature

t Time

U Internal energy

V Volume

V Specific volume

W Work performed by the headspace gas on the brine/waste

X Mole fraction of dissolved gas when the gas pressure equals one atmosphere 
$\mathrm{x} \quad$ Mole fraction of dissolved gas with any gas pressure

z Compressibility factor

\section{SUBSCRIPTS}

\begin{tabular}{|c|c|}
\hline adj & Adjusted value \\
\hline ave & Arithmetic average \\
\hline C & Container \\
\hline cal & Calibration value \\
\hline calc & Calculated value \\
\hline comp & Compensated value \\
\hline corr & Amount of correction \\
\hline $\mathrm{D}$ & Denotes a discrete event \\
\hline dis & Dissolved quantity \\
\hline GB & Glovebox \\
\hline g & Gas (in the headspace) \\
\hline gen & Result of gas generation \\
\hline $\mathrm{H}$ & High value for $\beta$ determination \\
\hline HS & Headspace \\
\hline $\mathrm{L}$ & Low value for $\beta$ determination \\
\hline 1 & Liquid (brine) \\
\hline $\mathrm{ME}$ & Mechanical energy \\
\hline RTD & Measurement by the resistance temperature detector in the brine or glovebox \\
\hline sampling & Resulting from sample extraction \\
\hline
\end{tabular}




$\begin{array}{ll}\text { sol } & \text { Resulting from solubility variation } \\ \text { s } & \text { Pressure transducer calibration } \\ \text { step } & \text { Resulting from a step change in the test container temperature } \\ \text { v } & \text { Vapor } \\ 0 & \text { Initial condition or reference value } \\ 1 & \text { Region defined in the thermal model } \\ 2 & \text { Region defined in the thermal model } \\ 3 & \text { Region defined in the thermal model } \\ \infty & \text { Region defined in the thermal model }\end{array}$

\section{GREEK SYMBOLS}

$\alpha \quad$ Linear coefficient of thermal expansion

$\beta \quad$ Volumetric coefficient of thermal expansion

$\Delta \quad$ Difference

$\delta \quad$ Small difference

$\gamma \quad$ Ratio of the heat capacity at constant pressure to the heat capacity at constant volume 


\title{
DEVELOPMENT AND APPLICATION OF AN ANALYSIS METHODOLOGY FOR INTERPRETING AMBIGUOUS HISTORICAL PRESSURE DATA IN THE WIPP GAS-GENERATION EXPERIMENTS
}

by

\author{
F. S. Felicione
}

\begin{abstract}
The potential for generation of gases in transuranic (TRU) waste by microbial activity, chemical interactions, corrosion, and radiolysis was addressed in the Argonne National Laboratory-West (ANL-West) Gas-Generation Experiments (GGE). Data was collected over several years by simulating the conditions in the Waste Isolation Pilot Plant (WIPP) after the eventual intrusion of brine into the repository. Fourteen test containers with various actual TRU waste immersed in representative brine were inoculated with WIPP-relevant microbes, pressurized with inert gases, and kept in an inert-atmosphere environment for several years to provide estimates of the gasgeneration rates that will be used in computer models for future WIPP Performance Assessments.
\end{abstract}

Modest temperature variations occurred during the long-term ANL-West experiments. Although the experiment temperatures always remained well within the experiment specifications, the small temperature variation was observed to affect the test container pressure far more than had been anticipated. In fact, the pressure variations were so large, and seemingly erratic, that it was impossible to discern whether the data was even valid and whether the long-term pressure trend was increasing, decreasing, or constant. The result was that no useful estimates of gasgeneration rates could be deduced from the pressure data. Several initial attempts were made to quantify the pressure fluctuations by relating these to the measured temperature variation, but none was successful.

The work reported here carefully analyzed the pressure measurements to determine if these were valid or erroneous data. It was found that a thorough consideration of the physical phenomena that were occurring can, in conjunction with suitable gas laws, account quite accurately for the pressure changes that were observed. Failure of the earlier attempts to validate the data was traced to the omission of several phenomena, the most important being the variation in the headspace volume caused by thermal expansion and contraction within the brine and waste.

A further effort was directed at recovering useful results from the voluminous archived pressure data. An analytic methodology to do this was developed. This methodology was applied to each archived pressure measurement to nullify temperature and other effects to yield an adjusted pressure, from which gas-generation rates could be calculated.

A review of the adjusted-pressure data indicated that generated-gas concentrations among these containers after approximately 3.25 years of test operation ranged from zero to over 17,000 ppm by volume. Four test containers experienced significant gas generation. All test containers that showed evidence of significant gas generation contained carbon-steel in the waste, indicating that corrosion was the predominant source of gas generation. 


\subsection{Introduction and Summary}

The long-term performance of the Waste Isolation Pilot Plant (WIPP) in effectively isolating contaminants from the environment is obviously very important to the success of the repository. Sandia National Laboratory (SNL) has the responsibility to make overall performance predictions. These predictions rest on having a good understanding of the behavior of the waste as it interacts with the repository environment, including the potential for migration of contaminants away from the site. A key parameter in these analyses is the predicted rise in repository pressure that may result from gases that are generated in the waste by microbial activity, radiolysis, corrosion, or other chemical reactions following the eventual intrusion of brine into the depository. The resulting increase in the internal pressure in the depository may exceed the lithostatic pressure exerted by the surrounding salt, affecting the calculated rates of migration of contaminants away from the repository. Assumptions made in the formal Performance Assessment calculations that are part of the WIPP licensing process need to be tested to ensure that conservative values were used. The Argonne National Laboratory-West (ANL-West) Gas Generation Experiment (GGE) Program was established to support this work by obtaining accurate data on the rates at which gases are generated in actual radioactive waste at prototypic pressure and temperature.

\subsection{Gas-Generation Experiments and Task Background}

The GGE was designed to assist SNL in estimating the rates at which gases are produced. The GGE was a very carefully constructed and controlled experiment. Fourteen specially manufactured, corrosion-resistant test containers having a nominal volume of about seven liters each were placed under test. In 12 of these approximately $75 \%$ of the container volume was loosely filled with various actual transuranic (TRU) waste. The other two test containers had no wastes and served as experiment controls. A synthetic brine having the same chemical composition as that in the WIPP vicinity was added, filling approximately $90 \%$ of the test containers' volumes. To include metabolic gas generation, all containers were inoculated with microbes found at WIPP. These test containers were then backfilled with nitrogen gas and pressurized to approximately $14.87 \mathrm{MPa}$ (2150 psia), the lithostatic pressure level expected to eventually occur at WIPP. Table 1 lists the types of waste materials within each of the test containers.

Table 1. Waste Contents Within Each Test Container

\begin{tabular}{|l|c|c|c|c|c|c|}
\hline $\begin{array}{c}\text { Container } \\
\text { Number }\end{array}$ & $\begin{array}{c}\text { Carbon Steel } \\
\text { Surface Area } \\
\left(\mathrm{cm}^{2}\left[\mathrm{in}^{2}\right]\right)\end{array}$ & $\begin{array}{c}\text { Aqueous } \\
\text { Sludge } \\
\text { Mass } \\
(\mathrm{g})\end{array}$ & $\begin{array}{c}\text { Leaded } \\
\text { Rubber Mass } \\
(\mathrm{g})\end{array}$ & $\begin{array}{c}\text { Rubber } \\
\text { (Neoprene) } \\
\text { Mass } \\
(\mathrm{g})\end{array}$ & $\begin{array}{c}\text { Plastic } \\
\text { (Polyethylene) } \\
\text { Mass } \\
(\mathrm{g})\end{array}$ & $\begin{array}{c}\text { Cloth \& Paper } \\
\text { (Cellulose) } \\
\text { Mass } \\
(\mathrm{g})\end{array}$ \\
\hline \hline VSL-151 & $\begin{array}{c}859.29 \\
(133.19)\end{array}$ & & 129.28 & & & \\
\hline VSL-152 & & & & & & \\
\hline VSL-153 & & & & & & \\
\hline
\end{tabular}


Table 1. (Contd.)

\begin{tabular}{|c|c|c|c|c|c|c|}
\hline $\begin{array}{c}\text { Container } \\
\text { Number }\end{array}$ & $\begin{array}{c}\text { Carbon Steel } \\
\text { Surface Area } \\
\left(\mathrm{cm}^{2}\left[\mathrm{in}^{2}\right]\right)\end{array}$ & $\begin{array}{l}\text { Aqueous } \\
\text { Sludge } \\
\text { Mass } \\
\text { (g) }\end{array}$ & $\begin{array}{l}\text { Leaded } \\
\text { Rubber Mass } \\
\text { (g) }\end{array}$ & $\begin{array}{c}\text { Rubber } \\
\text { (Neoprene) } \\
\text { Mass } \\
\text { (g) }\end{array}$ & $\begin{array}{c}\text { Plastic } \\
\text { (Polyethylene) } \\
\text { Mass } \\
\text { (g) }\end{array}$ & $\begin{array}{l}\text { Cloth \& Paper } \\
\text { (Cellulose) } \\
\text { Mass } \\
\text { (g) }\end{array}$ \\
\hline$\overline{\text { VSL-160 }}$ & $\begin{array}{c}926.51 \\
(143.61)\end{array}$ & 269.10 & 124.72 & 44.83 & 91.84 & 344.24 \\
\hline VSL-161 & $\begin{array}{c}904.39 \\
(140.18)\end{array}$ & 273.68 & 125.96 & 43.64 & 86.38 & 344.35 \\
\hline VSL-162 & $\begin{array}{c}929.74 \\
(144.11)\end{array}$ & 267.56 & 123.36 & 45.00 & 86.90 & 343.85 \\
\hline VSL-163 & & 271.62 & 125.52 & & & 345.22 \\
\hline VSL-164 & & 269.87 & 127.41 & & & 343.72 \\
\hline VSL-165 & & 285.10 & & 43.77 & 88.47 & \\
\hline VSL-166 & $\begin{array}{c}902.58 \\
(139.90)\end{array}$ & 275.63 & & & & \\
\hline VSL-167 & $\begin{array}{c}905.35 \\
(140.33)\end{array}$ & & & 42.52 & 88.43 & 345.56 \\
\hline VSL-168 & & & & & & 345.90 \\
\hline VSL-169 & & & & & & 341.83 \\
\hline VSL-170 & & & 123.03 & 43.48 & 88.80 & \\
\hline
\end{tabular}

All of the test containers were fitted with highly sensitive and stable temperature and pressure instrumentation, along with suitable systems for periodically extracting very small samples of the vessel headspace gas for chemical analysis. The test containers were placed in an inert-gas (argon)-filled glovebox having a sophisticated gas-purification system to restrict the level of oxygen present. Each test container was fitted with electric heater belts and had its own proportional, integral, and derivative (PID) electronic controller that controlled the temperature of each test container individually to nominally $30^{\circ} \mathrm{C}$, the temperature expected in the repository. A data-acquisition system acquired temperature and pressure measurements every hour. Regular calibrations were made to the instruments.

Chemical analyses of the gas samples over the testing period were intended to be used to deduce the rates at which gases were being produced and allow the specific gases generated to be identified. Pressure measurements were to provide a backup indication of gross gas generation. Actual testing began in 1996. By the end of the test program in the Fall, 2002, temperature and pressure data had been collected and archived for over six years, totaling in excess of 50,000 data points per container. (The analysis in this report addresses only the initial 3.25 years of operation, i.e., approximately the first 26,000 hourly data points per test container). 


\subsection{Operational Experience with the GGE}

The GGE tests were conducted within a cell that houses the now-shutdown Zero Power Physics Reactor (ZPPR) at ANL-West. Because of the substantial soil overburden on the reactor cell, the temperature within the reactor cell is relatively cool and tends to remain constant. In combination with the temperature control on each test container, there appeared to be no need to regulate the cell temperature in order to meet the temperature specification of $30 \pm 5^{\circ} \mathrm{C}$ that was imposed on the experiment. However, since the test containers can be heated, but have no cooling, the local environment needs to be below the $30^{\circ} \mathrm{C}$ target for the temperature controllers to be active. In fact, the slightly increased temperature within the glovebox that results from the operation of various experiment equipment causes the controllers to become inoperative if the reactor cell temperature exceeds about $28^{\circ} \mathrm{C}$.

The reactor cell is air conditioned, and this system can reduce the ZPPR-cell temperature by about $15^{\circ} \mathrm{C}$. It was found that long spells of hot summertime weather during the second year of test operation gradually increased the ZPPR-cell temperature above $26^{\circ} \mathrm{C}$ and required that the air conditioning be used. Temperatures within the test containers, themselves, were nonetheless maintained within a 2-to- $3^{\circ} \mathrm{C}$ band during all operation of the GGE, in full conformance with the test specifications. Despite the careful design, implementation, and control of the experiments, significant pressure fluctuations were measured. In fact, the fluctuations were so great and so irregular that the validity of the data was questioned, and it was impossible to deduce meaningful pressure trends.

The headspace gas was sampled periodically and analyzed by thermal-conductivity, gas chromatography. Although the gas samples extracted only a few milliliters of headspace gas, pressure decreases of 55-69 kPa (8-10 psi) occurred each time a sample was withdrawn from the small headspace volume $(650-750 \mathrm{ml})$ in each test container. After about one year of operation the puzzling fluctuations in the measured pressure were being recognized. By this time, six or seven headspace-gas samples had been taken from each test container and analyzed. Since each headspace-gas sample degraded the experiment pressure, further sampling was suspended, pending a full explanation of the pressure behavior.

\subsection{Initial Data Analyses}

Comparisons of the headspace pressure and temperature measurements showed a very obvious qualitative correlation in the direction expected: increases or decreases in temperature were consistently accompanied by increases or decreases, respectively, in the measured pressure. Classical gas laws relating pressure and temperature were applied, but these failed to account for even half of the pressure fluctuations measured. As an example, in a particular test container that had been operating at relatively steady conditions the heater set point was deliberately stepped up to a higher setting. The new conditions persisted long enough to allow the test vessel to reach a new steady state, and this caused the temperature to increase in the test container from about $29.6^{\circ} \mathrm{C}$ to $39.8^{\circ} \mathrm{C}$. The event was duly recorded by the data-acquisition system (Fig. 1). A simple ideal-gas pressure-temperature analysis would predict that at the new temperature the pressure should rise from the initial value of 14.615 MPa (2119.7 psia) to a new pressure of 


$$
P=P_{0} \frac{T}{T_{0}}=14.615 M P a \frac{39.8+273.15^{\circ} \mathrm{C}}{29.6+273.15^{\circ} \mathrm{C}}=15.107 \mathrm{MPa}(2191.1 \mathrm{pisa})
$$

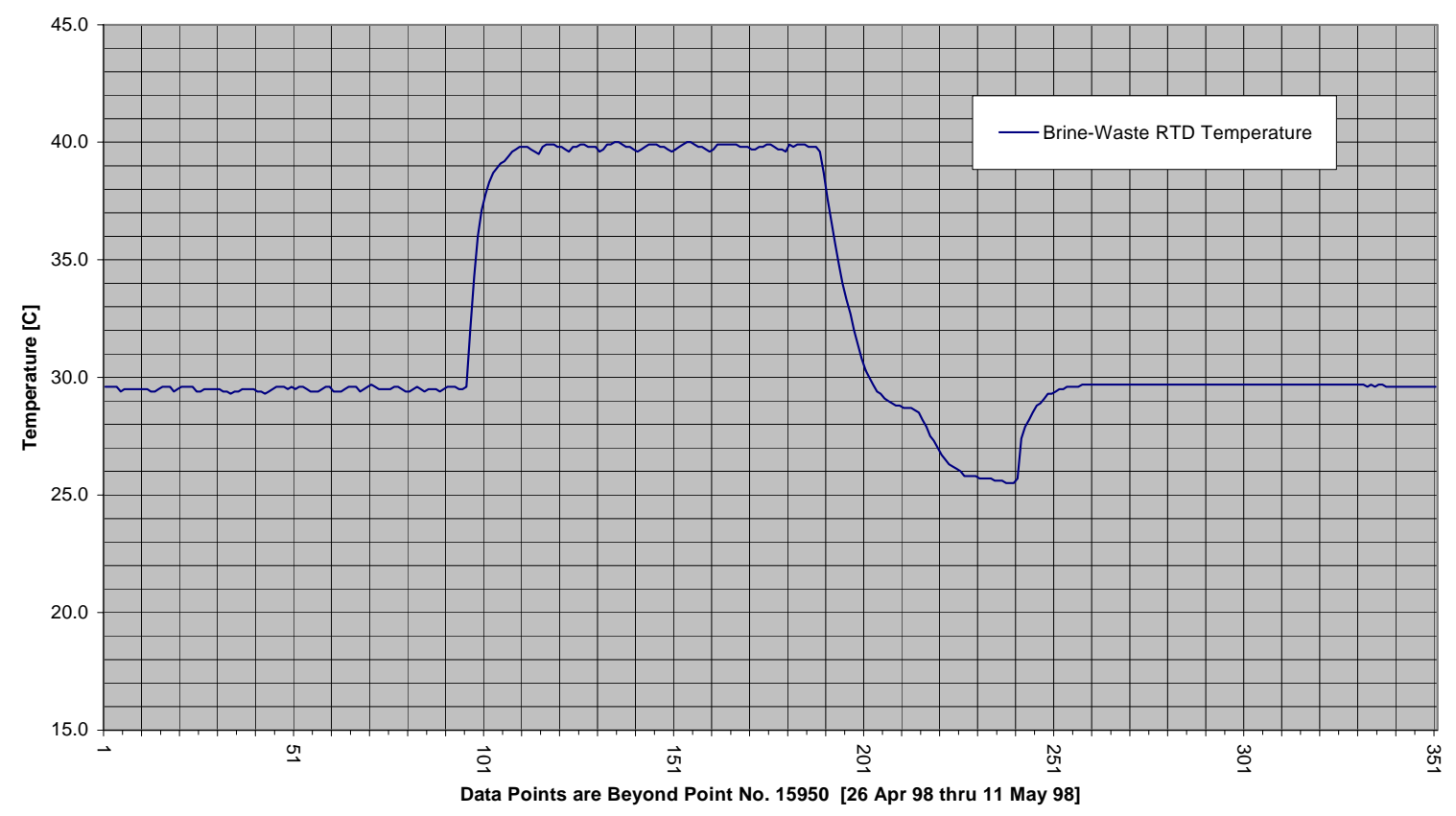

Fig. 1. Temperature Trace for a Step Increase in the Heater Setpoint (TC-153)

That is, a pressure rise of about $492.0 \mathrm{kPa}(71.4 \mathrm{psi})$. In fact, the measured pressure rise was actually about $1048.0 \mathrm{kPa}$ (152.0 psi), to $15.663 \mathrm{MPa}$ (2271.7 psia), as shown in Fig. 2, i.e., more than twice as high as the simple pressure-temperature relationship predicts. Application of the pressure-temperature relation to other data points proved equally unable to account for the measured pressure response.

These initial efforts failed to satisfactorily explain, much less quantify, the pressure behavior. Further reviews included attempts to apply empirical correction factors to the data, but with only marginal success, even for the blank, experiment-control containers. Worse still, these empirical approaches emphasized the inability to explain if, and how, the erratic pressure was related to any fundamental phenomenon, calling into question the overall validity of the data. Since the extensive data that had been collected appeared to be of little usefulness, the program sponsor initiated discussions concerning the early termination of the program. Termination of the program would deny the sponsor an important confirmation of assumptions used in the WIPP performance assessment models, as well as deriving no benefit from the considerable investment that had already been made to implement the GGE program. Obviously, early program termination would also eliminate a significant source of on-going funding to ANL-West. The need for a detailed understanding of the pressure fluctuations was clear, and this prompted the rigorous investigation reported here. 


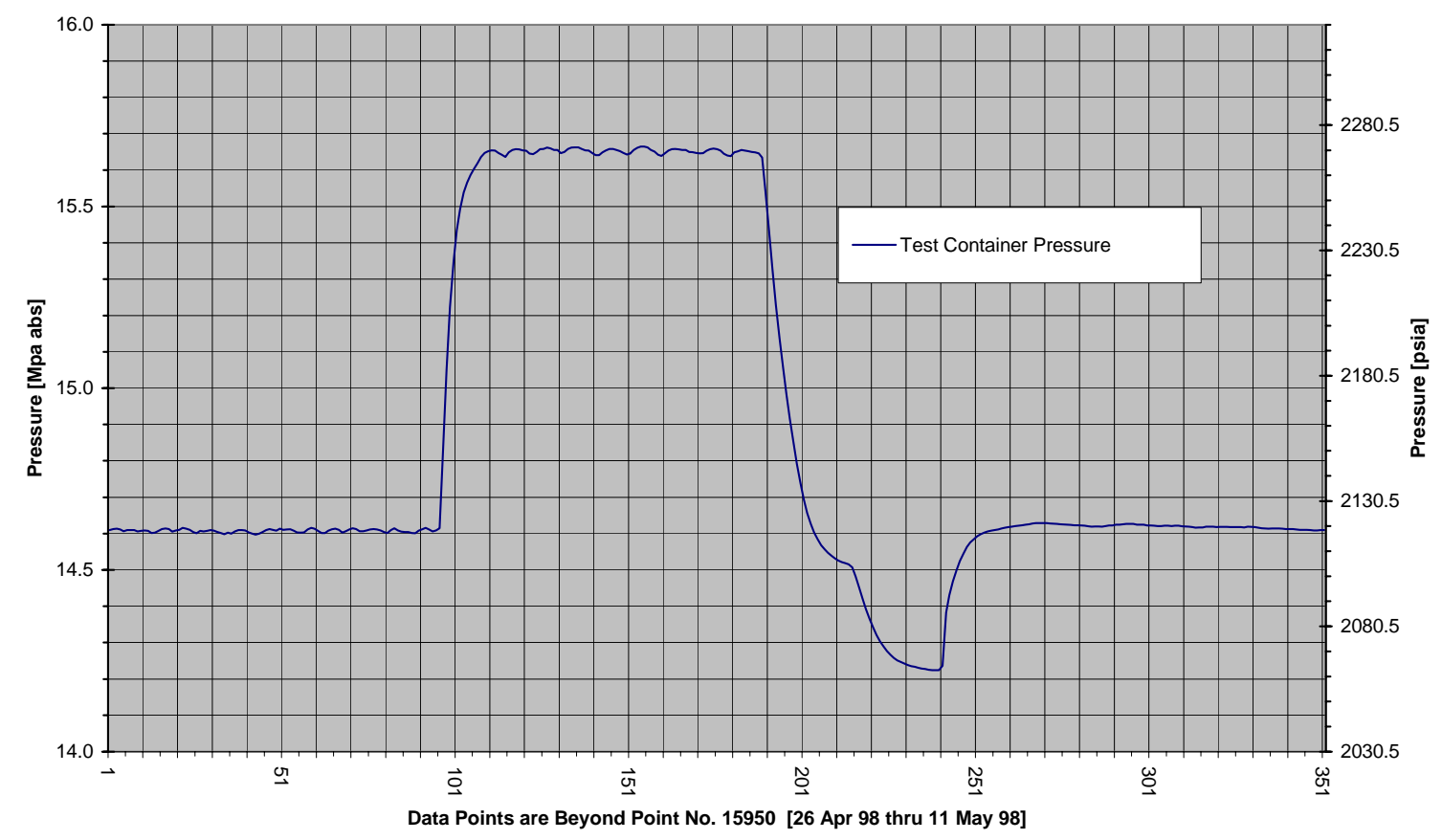

Fig. 2. Pressure Trace for a Step Increase in the Heater Setpoint (TC-153)

\subsection{Approach and Objectives of the Present Investigation}

\subsubsection{Validation of the Test Data}

The present investigation carefully examined the experiment equipment and its operation. A gaslaw analysis was made that included all of the potential contributors to pressure variation. The temperature distributions within the test containers (and, in particular, in the headspace), and temperature variations within the ZPPR cell and within the glovebox were considered. Thermal expansions and contractions of the test vessels, the brine, and the waste materials were evaluated. The vapor pressure (volatility) of the brine constituents, the solubility of the headspace gases in the brine and the accompanying variation in headspace-gas mass, and the compressibility of the brine and the waste were all examined. Corrections to account for changes and errors introduced during calibration of the temperature and pressure transducers were also developed and included, and the reductions in the headspace-gas mass from sample extractions were rigorously included.

Most of these phenomena could be analytically included using parameters available in the literature. A separate experimental apparatus was assembled and used to measure the thermalexpansion characteristics of the liquid brine. Using these parameters, the full analytic treatment was then applied to specific data collected from the experiment-control containers (those that contained brine and microbes, but no waste) to test the methodology. The measured pressure histories for these waste-free containers were as erratic as any, but the pressures that should prevail in the absence of variations in the temperature and other influences, should, of course, be constant. 
The full analysis was found to very accurately predict the effects of temperature variation on the pressure. This success with several data points clearly established the general validity of the data. The major shortcoming in the earlier analyses was traced to the constant headspace volume assumption, i.e., the omission of the effects of thermal expansion/contraction in the brine and waste. This was an unexpected and insidious phenomenon: the small temperature variations that occurred, generally just a few degrees Celsius, affect the volume of the brine/waste only very little. But volumetric changes in the brine/waste are directly impressed on the headspace volume. An increase or decrease in the brine and waste volume causes an equal decrease or increase, respectively, in the headspace-gas volume. Since the brine/waste matrix volume is much greater than the headspace volume, the relative volumetric variation in the headspace is amplified by more than an order of magnitude, as compared to that which occurs in the brine/waste. In fact, the brine/waste expansion/contraction phenomenon was found to be the predominant effect on test-container pressure, exceeding even the direct effect from variation of the headspace-gas temperature, itself.

\subsubsection{Recovery of the Archived Pressure Data}

Once it had been demonstrated that the causes of the pressure behavior were fully explainable and quantifiable, a method to nullify these effects in the several years of archived data for the waste-bearing containers was investigated. A key parameter to the methodology was the unknown sensitivity of the brine/waste volume to temperature changes. The method pursued involved deliberately introducing temperature steps in each test container and measuring the corresponding pressure response. This technique allowed the indirect determination of the thermal expansion-contraction characteristics of the brine and waste materials together, a parameter not otherwise known. This obviated the need to physically duplicate the brine/waste and measure the thermal-expansion characteristics in a separate experiment, as had been done for the brine alone, described in the previous section.

With the thermal-expansion characteristics for each waste container known, the full analytic methodology was programmed for computer application and then applied to all of the archived data for each test container. This produced a derived set of pressure-trend data from which the effects of variations in temperature, headspace-gas-mass variation from gas sampling and gas solubility variation, and instrument-calibration errors had been purged. These "adjusted" pressure trends made it possible to deduce the gas-generation rates.

\subsection{Organization of the Report}

This report describes the analytic investigation of the erratic pressure variation observed. Section 2.0 describes the GGE experiments, the hardware being used, and the general operation. Section 3.0 presents an overview of the data that had been collected. The analysis methodology is outlined in Section 4.0. Section 5.0 discusses the variability of the headspace-gas mass, and Section 6.0 discusses the headspace volume. The thermodynamic parameters for compressibility and temperature are developed in Section 7.0. Temperature distributions are discussed in Section 8.0. These effects are consolidated and a demonstration of the validity of the GGE data is presented in Section 9.0. Section 10.0 describes how the archived data was reconstructed, and Section 11.0 discusses the results and interprets these for evidence of gas generation. Section 12.0 discusses the applicability of the analytic adjustment of pressures to other 
experiment programs. Finally, a discussion of the limitations of the analysis is provided in Section 13.0. Back-up materials are provided in several appendices.

The work reported here was largely developed in the latter half of 1998 through Spring 1999, i.e., covering about the first 3.25 years of GGE operation. The calculation was then programmed for computation on a desktop computer. The methodology was outlined and the results were reported to the sponsor as they were developed. This served to resolve the question of data validity and provided the basis for continuation of the program. Complete documentation of the work by this report, along with minor refinements to the analyses, were conducted on and off at a relatively low-priority level from that time through 2002.

\subsection{GGE Experiment Configuration}

\subsection{Test Container Description}

Figure 3 was extracted from ANL Drawing No. W0421-0125-ED, Sheet 1 and shows a vertical cross section of the test containers. The test containers were designed for a maximum safe working pressure of $19.995 \mathrm{MPa}$ gage (2900 psig) at $30^{\circ} \mathrm{C}$. To minimize gas generation from corrosion of the test containers themselves (gas generation only from interactions among the brine and waste materials are the object of the tests) the bodies, top head, and all miscellaneous parts and fittings within the containers were fabricated from Hastelloy-C276 (Unified Material Number System (UNS) N10276). The diametrically split clamping ring, which has no contact with the brine/waste, was made from American Iron and Steel Institute (AISI) 4140 (UNS G41400) chromium-molybdenum steel. All penetrations into the vessel were through the top head. The numerous penetrations served several purposes: brine addition, sparging, headspace gas access, two thermowells for temperature-sensing instruments (resistance temperature detector [RTD]), and a fitting to connect to a rupture disk assembly for over-pressure protection. Twenty compression bolts clamp the head to the vessel body. A diamond-cross-section, Hastelloy-C276 metal gasket seals the head to the body. A perforated Hastelloy-C276 plate mounted on 50-mm-long standoffs hangs from the bottom of the vessel head to ensure that the waste materials remained fully submerged in the brine and cannot float into the headspace volume.

Wastes were loaded into the test containers in a separate area. The containers were transferred into the argon-filled GGE glovebox (described in the next section) where the inoculated brine was carefully added. The lids were fastened to the containers, and the containers were then purged for approximately $24 \mathrm{~h}$ with a 99\% nitrogen $\left(N_{2}\right)$, 1\% helium $(\mathrm{He})$ mixture injected through the sparging manifold at the bottom of the container to displace the argon. Following the purge, the containers were pressurized with the $N_{2} / \mathrm{He}$ mixture to $17.237 \mathrm{MPa}$ (2500 psia) and checked for leakage using commercial helium leak-detection equipment. After passing the leak check, the pressure was reduced to the WIPP lithostatic pressure of $14.824 \mathrm{MPa}$ (2150 psia), and the test period commenced. 


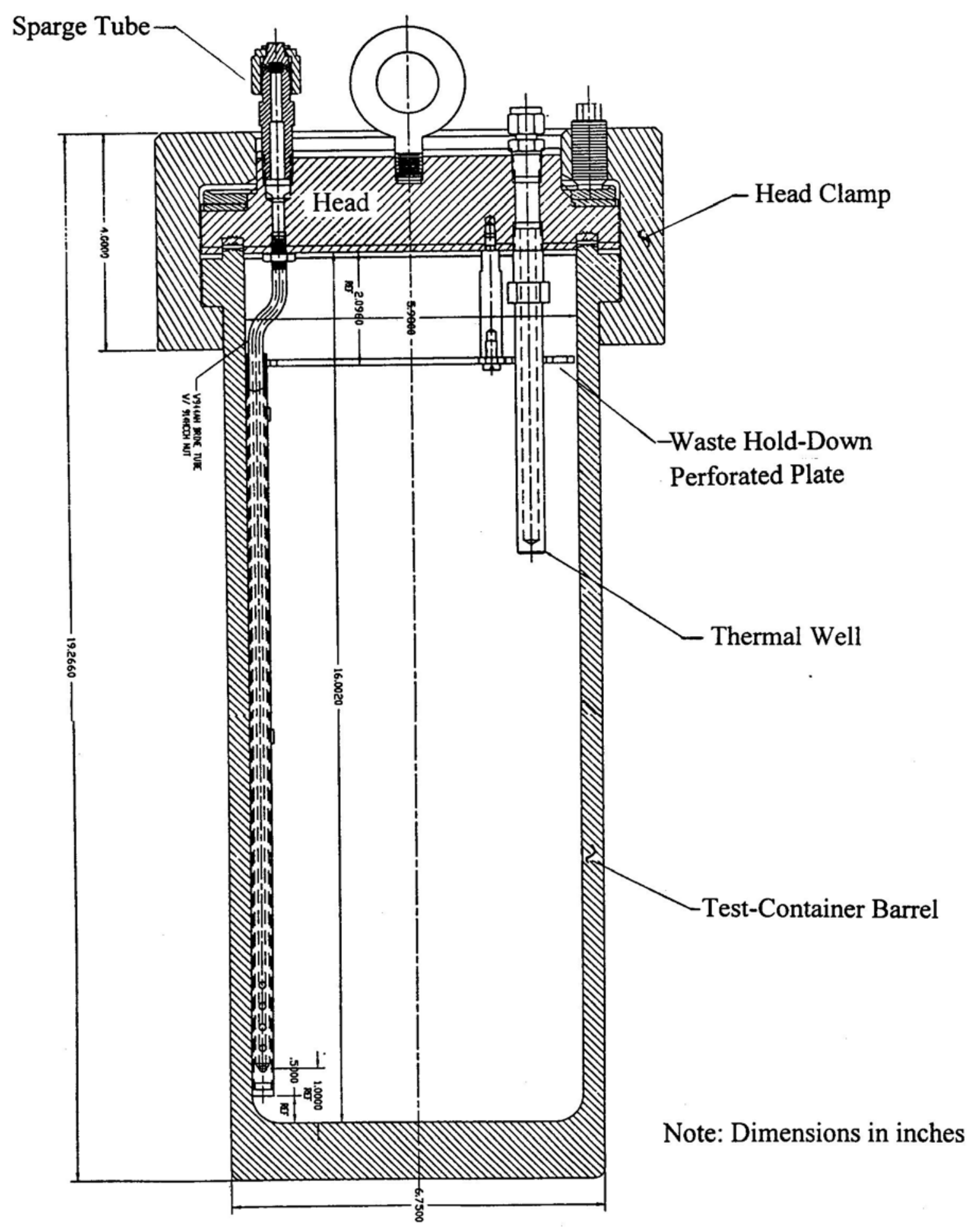

Fig. 3. Test Container Vertical Section 


\subsection{Glovebox}

The test containers were placed inside a large, controlled-atmosphere glovebox. Each test container's clamp ring rests on the upper end of a vertical, double-walled, cylindrical shroud that surrounds the test container. This arrangement keeps the underside of each test container approximately $15 \mathrm{~mm}$ ( $0.6 \mathrm{in}$.) above the floor of the glovebox. Three electric heater belts were mounted within the annulus of the double-walled shroud and wrapped tightly around the inner wall.

Figure 4 shows the heater configuration prior to installation of the outer wall of the concentric shroud. One heater belt provides ample power; the two additional belts were installed spares. The lowest belt was used initially for all test containers, and no failures were experienced.

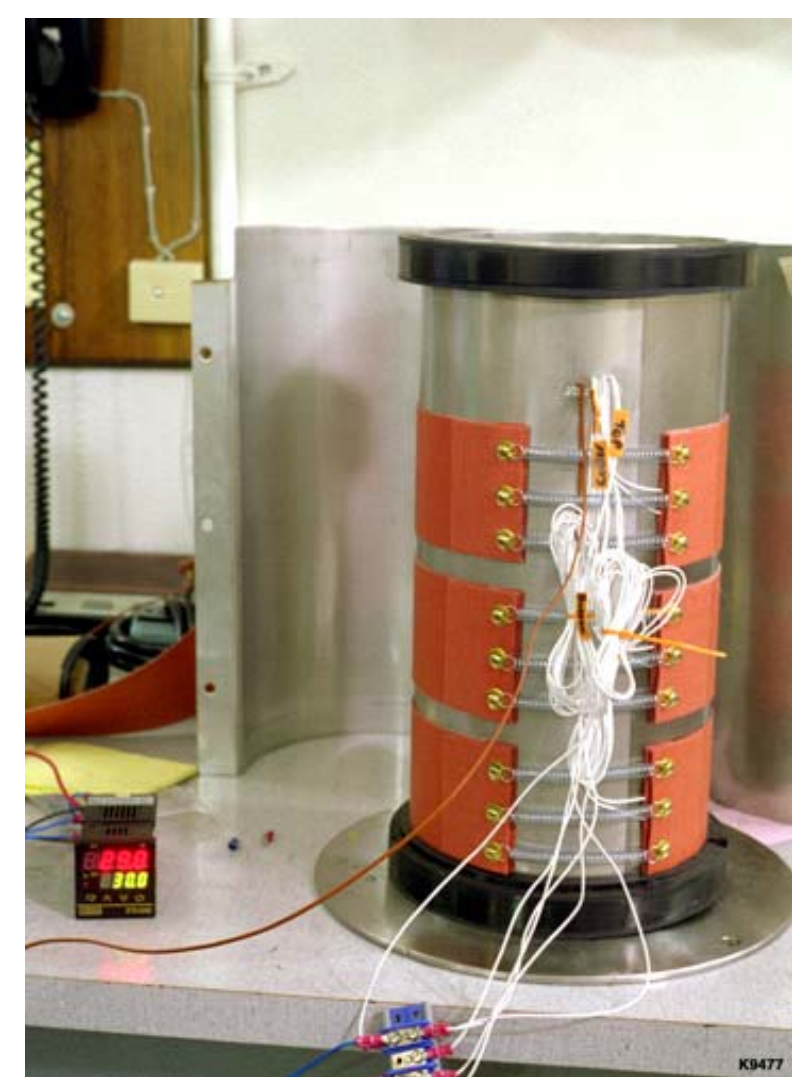

Fig. 4. Test-Container Shroud and Heater-Band Arrangement

When fully assembled, the test containers were as depicted in Fig. 5. The pressure is measured by an extremely accurate and stable pressure transducer mounted on a 3-mm-ID standoff tube above the container. The entire instrumentation connections are shown in Fig. 6.

The glovebox is shown in Fig. 7, extracted from ANL-West Drawing No. W0421-0030-ED. The test containers were surrounded by a false floor that had loose-fitting holes through which each test container assembly was placed. When in place, approximately the upper one-third of the test containers protruded above the false floor. Gas blowers circulated purified argon gas through the 
glovebox. The gas was circulated through two activated beds, alternately regenerated, to remove oxygen and other impurities. The temperature within the glovebox was measured by an RTD that resided in a pocket located underneath the false floor as shown in Fig. 7.

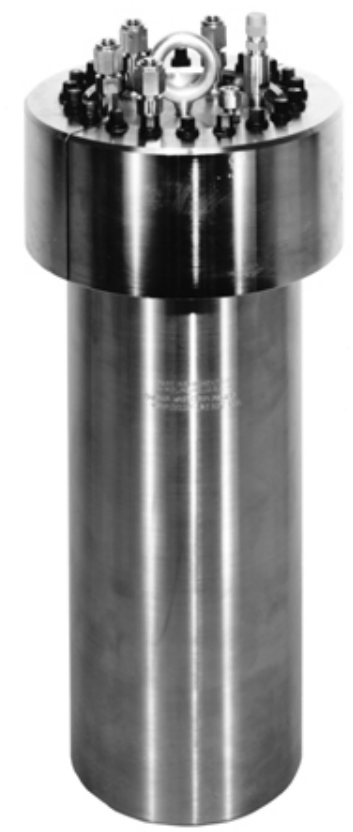

Fig. 5. Assembled Test Container 


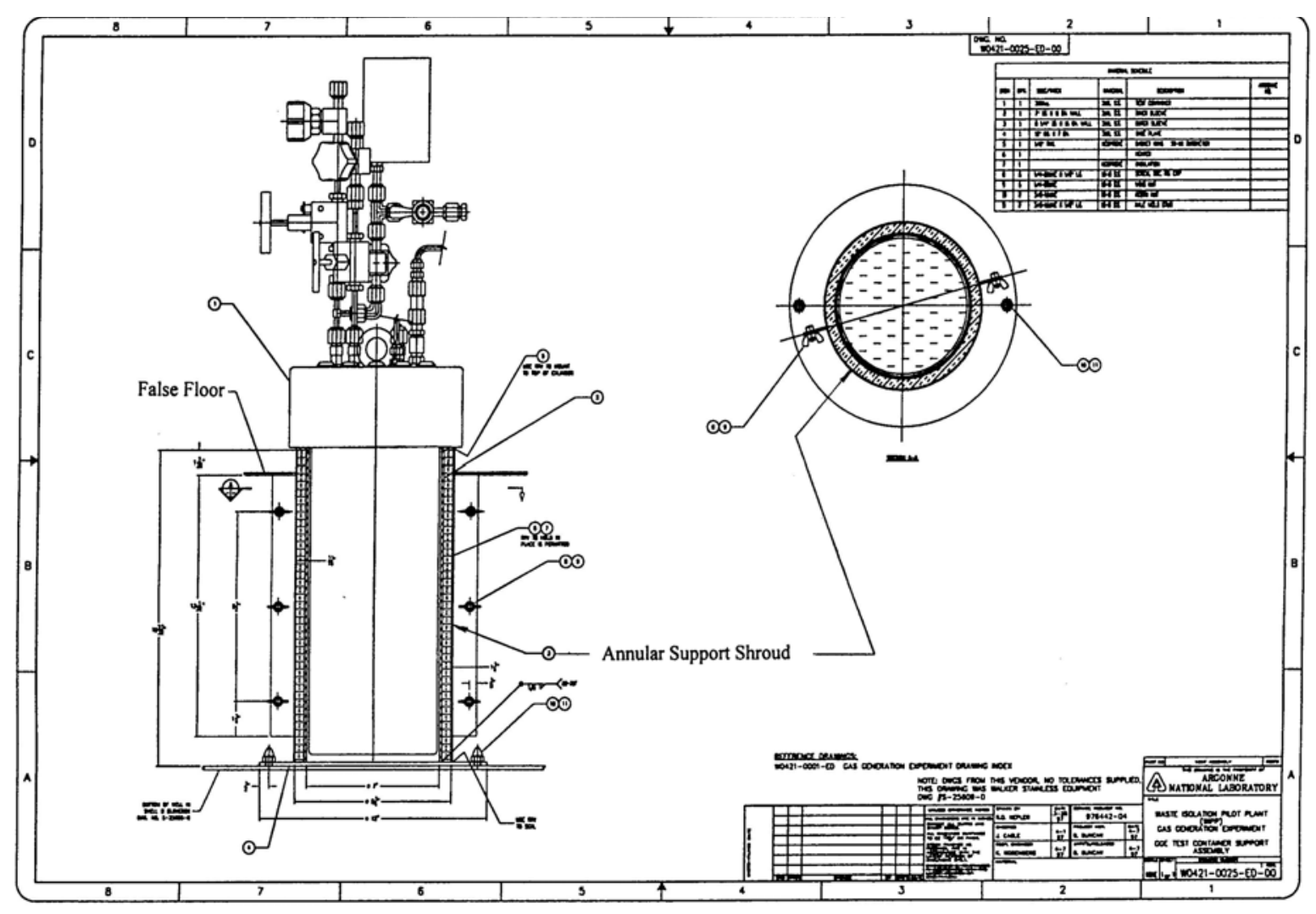

Fig. 6. Assembled Test Container in Glovebox with Instrumentation Connections
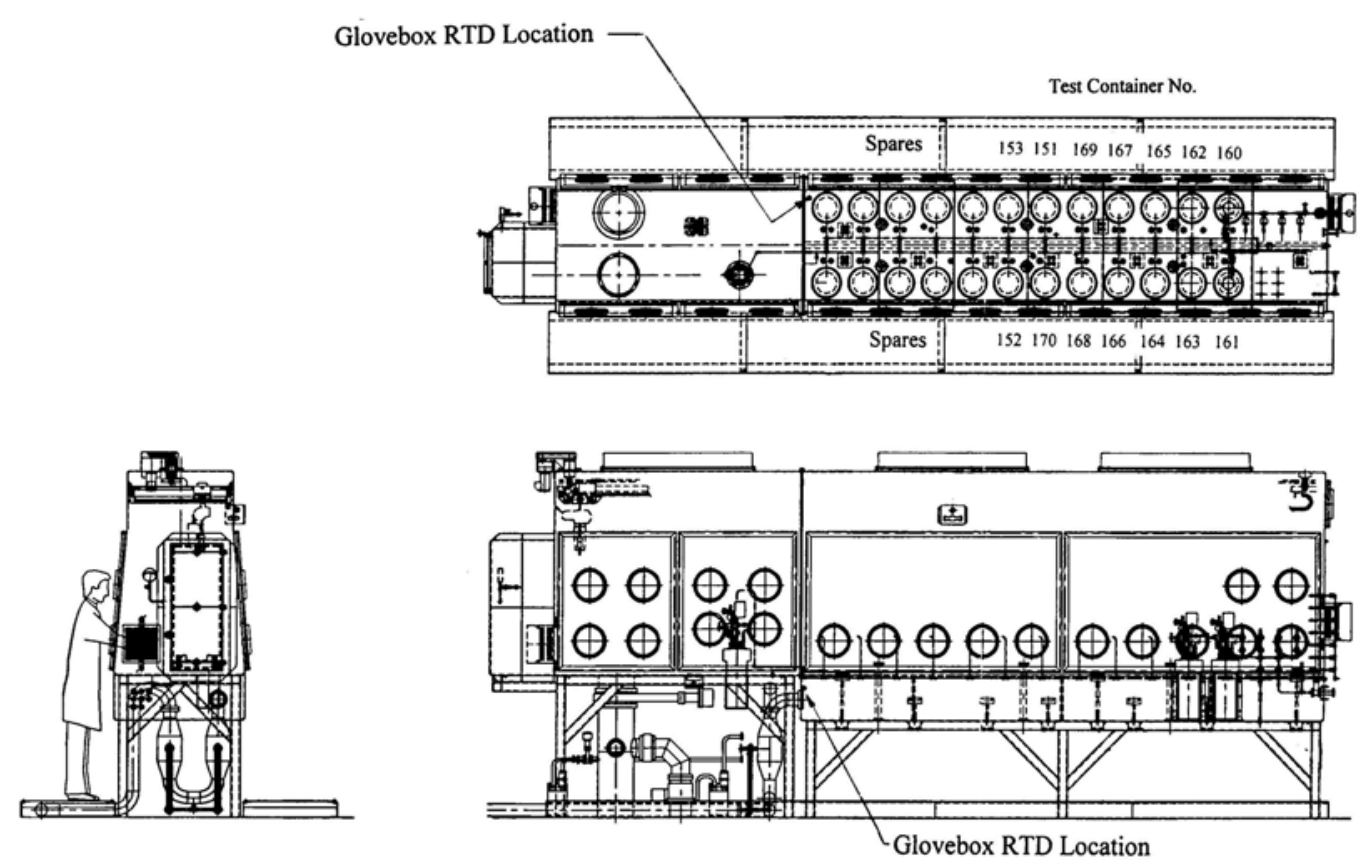

Fig. 7. GGE Glovebox Configuration 


\subsection{Overview of the Measured Data}

Examination of the data from a typical GGE test container is helpful in understanding the problem that was addressed. The temperature measured by the test-container RTD located in the thermowell shown in Fig. 3 is shown in Fig. 8. The deliberate increase in the test container temperature to about $39.8^{\circ} \mathrm{C}$, as was alluded to in Section 1.3 and Fig. 1 and Fig. 2, can be seen in Fig. 8 at about Data Point No. 16000. Figure 8 also shows the trace of the temperature measured by the glovebox RTD (see Fig. 7 for the glovebox RTD location). The ZPPR cell air conditioning was turned off at the beginning of the GGE tests, and the rise in the facility and glovebox temperatures are evident. After about 16 months and an unexpectedly hot summer, the facility air conditioning was turned back on (Point No. 2), and its effect in rapidly lowering the ZPPR cell temperature and the glovebox temperature is clearly evident. Prior to the activation of the cell air conditioning, the temperature of the glovebox and test container are clearly synchronized, time wise. Furthermore, it is evident that between Points No. 1 and No. 2 the glovebox temperature had risen so high that the test-container temperature was merely following that of the glovebox, i.e., the test-container's heater controller was no longer active. Following the activation of the ZPPR cell air conditioning at Point No. 2, the lowered glovebox temperature allowed the heaters to regain control of the test-container temperature. Several other activations/deactivations of the ZPPR cell air conditioning are also shown on Fig. 8. Data from this one test container is characteristic of all fourteen GGE test containers.

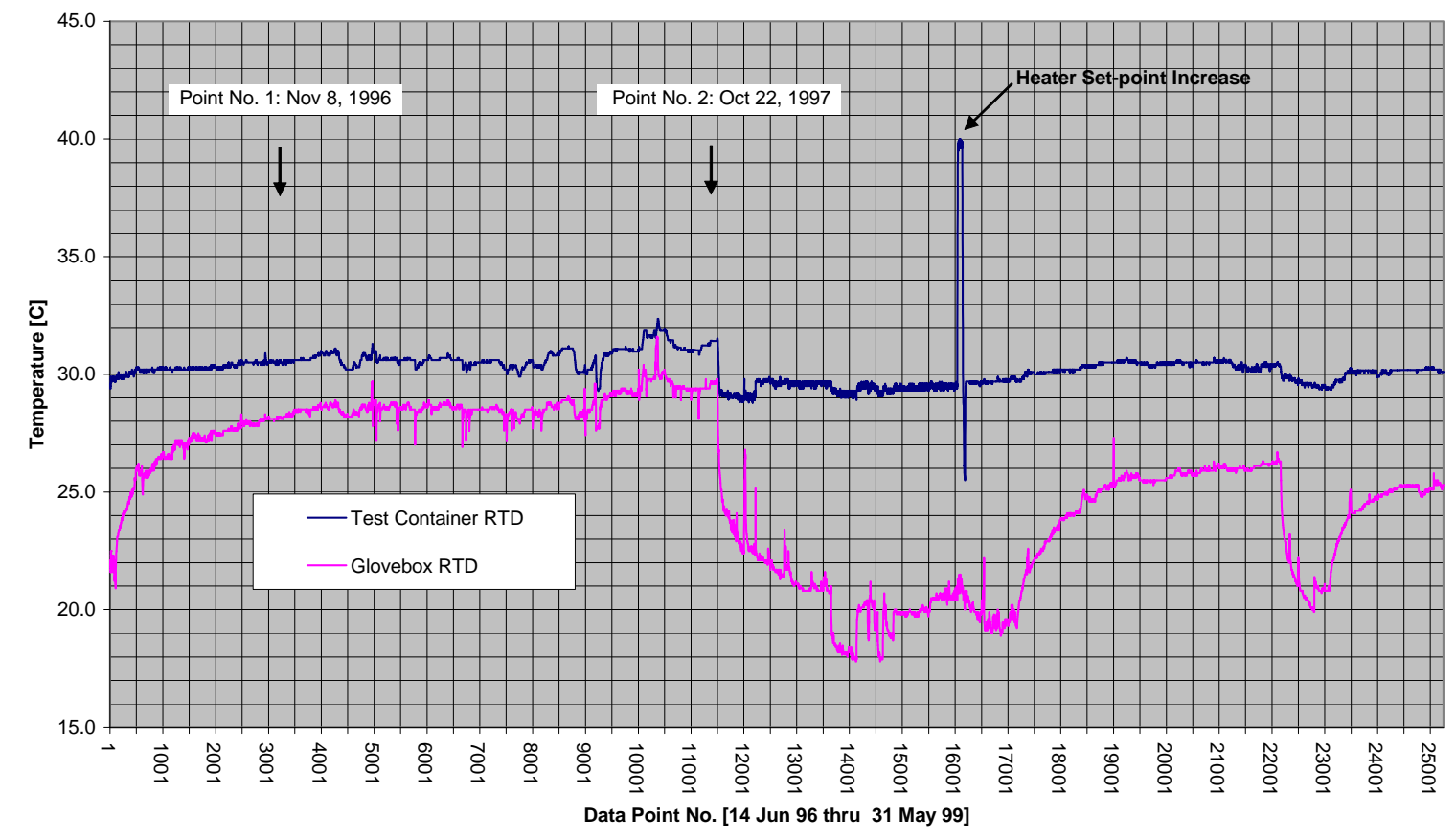

Fig. 8. Test Container and Glovebox Temperatures for a Typical Test Container (TC-153)

Figure 9 shows the temperature trace from this test container's RTD superimposed on a common abscissa with the pressure trace from the same test container. It can be seen from this plot, as well as in Fig. 1 and Fig. 2, that there is certainly a qualitative relationship between the pressure and 
the temperature. Determination of the quantitative relationship and the cancellation of any temperature and headspace-gas-mass effects were the objectives of the analysis methodology described in the next several sections of this report.

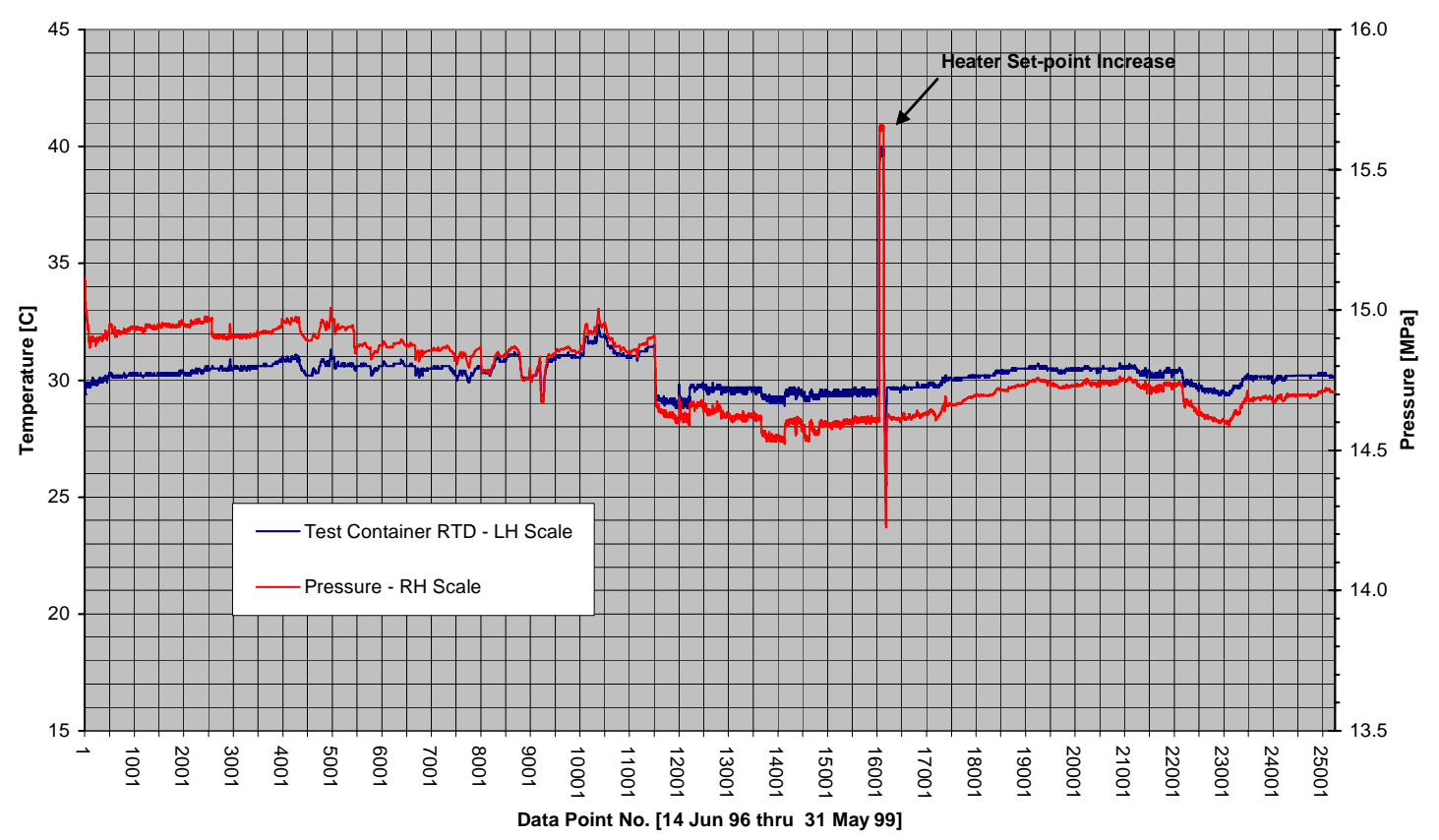

Fig. 9. Synchronization of Test Container Pressure with Temperature (TC-153)

\subsection{Analysis Development}

\subsection{Application of the State Equation}

The GGE test containers were backfilled with nitrogen. A small quantity of helium was added for use as a tracer during leak testing. At normal atmospheric pressure, these gases behave very nearly like thermally perfect gases. However, at increasingly higher pressures, a small deviation occurs. This can be accounted for by the inclusion of a correction, or "compressibility", factor, $z$, so that an equation of state can be expressed in a form similar to that of a perfect gas as

$$
P V_{H S}=z M R T=z N R_{U} T
$$

$P$ is the pressure, $V_{\mathrm{HS}}$ is the headspace volume, $M$ is the headspace-gas mass, and $T$ is the absolute temperature. The second form of the equation states the quantity of headspace gas in "moles", $N$, where the number of moles is the mass divided by the molecular mass (or molecular "weight” [MW]):

$$
N=\frac{M}{[M W]}
$$

Expressing the state equation in moles is more useful, since the corresponding gas-constant, $R_{\mathrm{U}}$, is independent of the particular gas species. The mass-specific gas constants for the generated 
gases are markedly different from that for the $N_{2}$ bulk fill gas. The predominant generated gas expected is hydrogen. Hydrogen is generated from metal corrosion as well as from the radiolysis of organic waste matter and water. Other potentially generated gases, such as $\mathrm{CO}_{2}$ and $\mathrm{H}_{2} \mathrm{~S}$ from microbial metabolism, ultimately attack the abundant carbon steel or aluminum wastes, so they too eventually cause the formation of $\mathrm{H}_{2}$. Since the molecular weight of $\mathrm{H}_{2}$ is so low, approximating its mass-specific gas constant by that for $N_{2}$ would not be acceptable, requiring the use of an averaged value, continually changing as the gas composition changed. Although the headspace gas will be quantified in moles and written as $N$ throughout this report, the familiar term "mass" is used when referring to the quantity of headspace-gas matter.

The state equation allows any one parameter to be determined when the others are known. Another way to invoke the state equation is to write Eqn. (2) for one set of conditions and divide that equation by the same equation written for a second set of conditions. This allows, for example, the pressure to be expressed in terms of a known pressure. Given a set of arbitrarily selected reference conditions for which the parameter values are known, denoted by a zero subscript, the pressure at another set of conditions (those without subscripts) is

$$
P=P_{0}\left(\frac{Z}{Z_{0}}\right)\left(\frac{N}{N_{0}}\right)\left(\frac{V_{0}}{V}\right)\left(\frac{T}{T_{0}}\right)
$$

Evaluation of the right-hand side of Eqn. (4) will predict the pressure at any point when the reference pressure, $P_{0}$, and the ratios of the four other thermodynamic parameters have been accurately specified. In particular, for each GGE test container, a point-by-point evaluation of Eqn. (4) with the corresponding parameter ratios will predict the actual pressure history relative to some reference pressure. The pressure behavior determined this way can be compared to the pressure history recorded by the data-acquisition system. A suitable match validates the data; a mis-match would indicate that either the data is invalid, the theory is incorrect or incomplete, or that the parameters have not been properly specified.

An equivalent way to apply the methodology is to rearrange Eqn. (4) to give the reference pressure in terms of the measured pressure as

$$
P_{0}=P\left(\frac{Z_{0}}{Z}\right)\left(\frac{N_{0}}{N}\right)\left(\frac{V}{V_{0}}\right)\left(\frac{T_{0}}{T}\right)
$$

With this formulation, when the measured pressure at any data point, $P$, and the other parameters are substituted into Eqn. (5) the selected reference pressure, $P_{0}$, should be found. The error between the calculated estimate and the actual (constant) reference pressure is an indicator of the accuracy of the methodology. This is the form used in this report and will be developed further in subsequent sections. 


\subsection{Analysis Approach}

Each of the terms in Eqn. (4) or (5) refers to properties of the headspace gas, and subsequent reference to these terms in this report have a $g$ (gas) subscript appended to these terms to differentiate these from the corresponding parameters in the brine/waste wherever ambiguity may otherwise result (e.g., temperature). The value of each of these terms at any point in time depends on the transient response of the test containers to variations in their surroundings, such as temperature changes in the ZPPR cell, in the glovebox, or heater activation. When any such change occurs, the test containers will begin to respond, gradually adjusting to whatever the new conditions and the physics of the system dictate. However, since the time frames of these external variations are rather long, on the order of several hours, the lag between these external "forcing functions" and the test container response should be small. For this reason, all parts of the test containers are presumed to be in constant and complete thermal equilibrium with their surroundings at all time. This assumption permits the response of the headspace gas to be analyzed as a succession of steady states, instead of calculating the actual transient response. That is, for a set of external forcing functions, the parameters in the headspace gas are presumed to be fixed and can be calculated as if the external conditions at any given instant had prevailed indefinitely. The genesis of the new state is simply discarded. Consistent with this approach, only the comparison of the external conditions to those at some arbitrarily chosen, fixed "reference" time need be known to determine the current state. This is an important characteristic of the present analysis. It will be shown, however, that this simplification does not impose serious limitations for the current application.

Having greatly simplified the problem by adopting a quasi-steady approach, the resolution of the GGE pressure behavior is reduced to an evaluation of Eqn. (5) for each data point for each of the test containers. The process is straightforward and ostensibly quite simple, but requires meticulous attention to the physics to properly account for all of the important phenomena that are occurring. The challenge in performing the analysis stems from the fact that, aside from the pressure, none of the parameters in Eqn. (5) was directly measured. Each ratio must be deduced from the only measurements that were made: the headspace total pressure and the brine/waste and glovebox temperatures. Accordingly, the majority of this report concerns the development of suitable expressions for the mass of headspace gas, $N$, the headspace volume, $V_{\mathrm{HS}}$, the headspace-gas compressibility, $z$, and the headspace-gas temperature, $T_{\mathrm{g}}$. Several appendices provide supporting materials.

\subsection{Headspace-Gas-Mass Considerations}

The quantity of gas within the headspace plays a significant role in the analyses. Expressions for this parameter are developed in this section.

\subsection{Headspace-Gas Composition and Volatility}

The headspace in each test container was backfilled with gas having a volumetric composition of approximately 99\% nitrogen and 1\% helium. Traces of other gases, principally argon, remained from handling the open test containers and gas-sampling operation in the argon-filled glovebox despite extensive purging with the nitrogen/helium fill gas after the containers were sealed. Equations (4) and (5) are applicable to each of the gas species present in the headspace when $z$ 
and $N$ refer only to that particular specie, and $P$ is the partial pressure of that specie. The measured pressure is the sum of all partial pressures. Inclusion of the actual initial gas composition is straightforward, but yields results that are virtually indistinguishable from those found by approximating all of the headspace gas simply as nitrogen. Since the additional calculational complexity from treating gas species separately serves no useful purpose, the headspace gas was subsequently approximated as 100\% nitrogen, and properties and parameters for the headspace gas are those for nitrogen in the remainder of this report.

In addition to gas species, there will be some vapor present in the headspace from the vaporization/condensation of the brine. The full pressure is the sum of the partial pressures of all of the gases, both condensable and non-condensable (non-condensable refers to the behavior at room-like temperatures). The subscript $g$ refers to the gas (approximated here as pure nitrogen), and the subscript $v$ refers to the condensable vapor, i.e.,

$$
P=P_{g}+P_{v}
$$

For modest rates of temperature change the headspace will continually be saturated with vapor at the partial pressure corresponding to the local vapor pressure. Water vapor is the only appreciable condensing species at room-like temperatures, so the partial pressure of the vapor, $P_{\mathrm{v}}$, can be found immediately for a given temperature from saturation data for water, viz., the "steam tables" [1]. Since the partial pressure of the gas specie is equal to the measured pressure minus the partial pressure of the vapor, Eqn. (4) can be applied directly to the non-condensing gas species (nitrogen), as

$$
P_{g}=P-P_{v}=\left(P-P_{v}\right)_{0}\left(\frac{z}{Z_{0}}\right)\left(\frac{N}{N_{0}}\right)\left(\frac{V_{H S_{0}}}{V_{H S}}\right)\left(\frac{T_{g}}{T_{g_{0}}}\right)
$$

The volume in Eqn. (7) is the total headspace volume, and the gas and vapor in the headspace are assumed to be in equilibrium at a common temperature. Similarly, Eqn. (5) can be written

$$
P_{0}-P_{v_{0}}=\left(P-P_{v}\right)\left(\frac{Z_{0}}{Z}\right)\left(\frac{N_{0}}{N}\right)\left(\frac{V_{H S}}{V_{H S}}\right)\left(\frac{T_{g_{0}}}{T_{g}}\right)
$$

The actual headspace gas mass at any point in time probably differs from that at the reference point. The totality of the increase in the headspace-gas mass relative to the mass at the reference point is written as $\Delta N$. Therefore, the headspace-gas mass at any specific point being analyzed is

$$
\begin{aligned}
N= & N_{0}+\left(N-N_{0}\right) \\
= & N_{0}+\Delta N
\end{aligned}
$$

The mass ratio in Eqn. (8) can then be written as

$$
\frac{N_{0}}{N}=\frac{N_{0}}{N_{0}+\Delta N}
$$


Once $\Delta N$ has been determined, this ratio can be calculated. The remainder of this section considers the various phenomena that produce variation, $\Delta N$, in the headspace-gas mass.

\subsection{Sources of Variation in the Headspace-Gas Mass}

The mass of gas within the headspace can vary for several reasons. There are two types of variation that the test containers are subjected to that are discrete, i.e., occurring only at specific times: (1) at each headspace-gas-sampling a small quantity of gas is physically extracted from the headspace for analysis; and (2) because of the particular technique required, the periodic calibration of the pressure transducer (PXD) introduces a potential for inadvertently altering slightly (either increasing or decreasing) the headspace-gas mass. A less obvious mass change may occur at any time as a result of variation in the quantity of headspace gases dissolved in the brine. Solubility of a given gas in the brine depends on the pressure of the gas species in the headspace and the brine temperature. Solubility variation acts like a source or sink of gas to the headspace. Finally, of course, there may have been gases generated within the brine as a result of microbial metabolism, radiolysis, or corrosion. Solubility variation and gas generation are continuous changes, or at least piece-wise continuous over short segments of time.

The total change in headspace mass can be resolved into its contributors, per the above discussion:

$$
\Delta N=\Delta N_{\text {sampling }}+\Delta N_{p x d}+\Delta N_{\text {sol }}+\Delta N_{g e n}
$$

Each of these terms represents the total increase (algebraically summed) with time in the headspace-gas mass as a result of sample extractions, PXD calibrations, solubility variation, and gas generation, respectively, between the current point and the reference point. With this expression, Eqn. (8) becomes

$$
P_{0}-P_{v_{0}}=\left(P-P_{v}\right)\left(\frac{z_{0}}{z}\right)\left(\frac{N_{0}}{N_{0}+\Delta N_{\text {sampling }}+\Delta N_{p x d}+\Delta N_{\text {sol }}+\Delta N_{g e n}}\right)\left(\frac{V_{H S}}{V_{H S}}\right)\left(\frac{T_{g_{0}}}{T_{g}}\right)
$$

Each of the contributors to the mass increase, except for the gas-generation, can be evaluated from conditions measured in the experiment. Expressions for the difference (increase) in headspace gas mass between a given point and the reference point for each of the non-gasgeneration components are developed in the following sections.

\subsubsection{Discrete Headspace-Gas-Mass Variation}

The two discrete-change components of $\Delta N$, i.e., from gas sampling and the PXD calibrations, can be written, for brevity, as a single term, $\Delta N_{\mathrm{D}}$, where by definition,

$$
\Delta N_{D}=\Delta N_{\text {sampling }}+\Delta N_{P X D}
$$

Gas sampling and PXD calibrations occur only at specific discrete points, where a discrete change of magnitude $\delta N_{\mathrm{D}-\mathrm{i}}$ is experienced. This term is algebraic: always negative for a sampling 
event, and either positive or negative for a PXD-calibration event. Consider the relationship between $\Delta N$ and $\delta N$ for some hypothetical sequence of headspace-gas-mass changes that result from any discrete phenomenon. For example, between Point- $A$ and the reference point in Fig. 10 there are two mass additions (events 1 and 2) and two mass extractions (events 3 and 4), having arbitrary magnitude. Similar headspace-gas-mass-change events occur at points beyond the reference point to some Point- $B$. Starting from Point- $A$, it is easily seen that

$$
N_{A}+\delta N_{4}+\delta N_{3}+\delta N_{2}+\delta N_{1}=N_{0}
$$

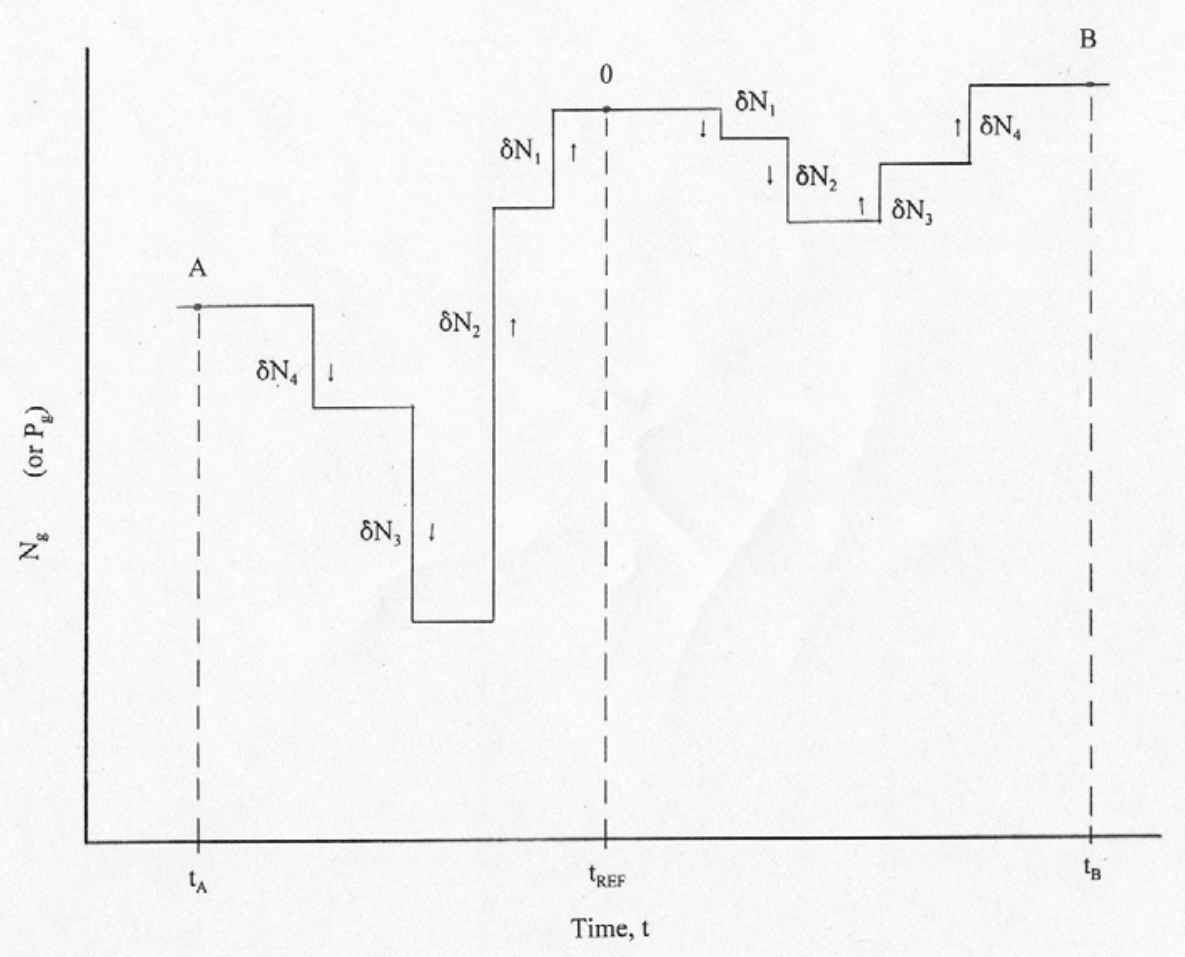

Fig. 10. Representation of Discrete Events at Times Prior To and Beyond the Reference Point

The quantities $\delta N_{4}$ and $\delta N_{3}$ are decreases (negative); $\delta N_{2}$ and $\delta N_{1}$ are increases (positive). Comparing Eqn. (14) to Eqn. (9), at points prior in time to the reference point, the difference between headspace-gas mass at a given point and that at the reference point is

$$
\Delta N=N_{A}-N_{0}=-\Sigma \delta N_{i}
$$

Similarly, beyond the reference point to Point- $B$ an analogous development shows

$$
N_{0}+\delta N_{1}+\delta N_{2}+\delta N_{3}+\delta N_{4}=N_{B}
$$

In this case, 


$$
\Delta N=N_{B}-N_{0}=+\Sigma \delta N_{i}
$$

Generalizing this to the case where there are a total of $J$ discrete events that occur between some point and the reference point,

$$
\Delta N_{D}=\mp \sum_{i=1}^{J} \delta N_{D_{i}}
$$

For points prior in time to the reference point the negative sign is to be used; the positive sign applies to points beyond the reference point.

The discrete events occur nearly isothermally, and typically involve only very small pressure changes, on the order of $55-69 \mathrm{kPa}$ (8-10 psi), a variation of less than $0.5 \%$ of the $14.824-\mathrm{MPa}$ (2150-psia pressure). The variation in compressibility is even smaller (on the order of $0.03 \%$ ). The mass change over the $i^{\text {th }}$ such discrete event can therefore be found by expressing the mass terms with the equation of state, Eqn. (2), and differentiating, giving

$$
\delta N_{D_{i}}=\frac{V_{H S} \delta P_{g_{i}}}{z R_{U} T_{g}}-\frac{P_{g} V_{H S} \delta z}{z^{2} R_{U} T_{g}} \approx \frac{V_{H S} \delta P_{g_{i}}}{z R_{U} T_{g}}
$$

Pressures and temperatures were recorded during the discrete events. Use of these pressures in Eqn. (19) allows the determination of the mass change at each discrete event once $V_{\mathrm{HS}}$ is known. The headspace volume that pertains to each discrete event for use in Eqn. (19) is discussed in Section 6.0.

\subsubsection{Continuous Headspace-Gas-Mass Variation}

Variation in the headspace-gas mass as a result of continuous (subscript $C$ ), or piece-wise continuous, changes are not intrinsically different than discrete changes. In the most general case, over some specific time interval, $\delta t_{\mathrm{i}}$, a change in the headspace-gas mass occurs of magnitude $\delta N_{\mathrm{C}-\mathrm{i}}$, where

$$
\delta N_{C_{i}}=\int_{\delta t_{i}} d N_{C}=\int_{\delta t_{i}} \frac{d N_{C}}{d t} d t=\int_{\delta t_{i}} \dot{N} d t
$$

If there are $K$ intervals over which continuous changes occur, then by analogy to the preceding section

$$
\Delta N_{C}=\mp \sum_{i=1}^{K} \delta N_{C_{i}}
$$


As was the case for the discrete variation, the negative sign is used for intervals prior to the reference point and the positive sign is used beyond the reference point.

The values of $J$ and $K$ in Eqn. (18) and Eqn. (21) refer only to the number of headspace-gas mass-change events that actually fall between the calculational point being considered and the reference point. Therefore, the values for each of $J$ and $K$ are unique to each point, and those values at one calculational point will likely differ from those at other points.

\subsubsection{Gas-Solubility Variation}

By definition, at any gas pressure the mole fraction of a dissolved gas (subscript dis) in a liquid (subscript $l$ ) is

$$
x=\frac{N_{d i s}}{N_{l}+N_{d i s}}
$$

The increase in headspace-gas mass equals the decrease, i.e., the negative of the increase, in the mass of the dissolved gas. Thus, the solubility-variation contribution to the overall gas-mass variation in the headspace is given by the negative of the difference between the dissolved gas mass at the current calculational point and the dissolved-gas mass at the reference point. Using this,

$$
\Delta N_{\text {sol }}=-\Delta N_{d i s}=-\left(N_{d i s}-N_{d i s_{0}}\right)=N_{d i s_{0}}-N_{d i s}
$$

The dissolved gas quantity can be computed from Eqn. (22), so that

$$
\Delta N_{\text {sol }}=N_{l}\left(\frac{x_{0}}{1-x_{0}}-\frac{x}{1-x}\right)
$$

The equilibrium quantity of gas dissolved can be determined using solubility relationships found in the literature. For a given gas/solvent combination, Reference 3 gives the mole fraction of gas dissolved in the brine, $X_{\text {dis }}$, when the partial pressure of that gas above the liquid surface is one atmosphere as

$$
\log _{e} X_{d i s}=A+\frac{B}{T^{\prime}}+C \log _{e} T^{\prime}
$$

The term $T^{\prime}$ is a non-dimensional temperature defined by $T^{\prime}=T / 100 \mathrm{~K}$, and $A, B$, and $C$ are gas/ solvent-specific constants. For nitrogen gas in water, the constants $A, B$, and $C$ are -67.3877 , 86.3213, and 24.7981, respectively (3). Nitrogen is the predominant gas in the headspace. Although there are much more soluble gases that may be present as a result of gas generation, e.g., $\mathrm{CO}_{2}$ or $\mathrm{H}_{2} \mathrm{~S}$, these would be expected to be present in such limited quantities that even 
significant variation in their solubilities would have only a minor effect on the total headspacegas mass. These values were presumed to pertain to solutions of nitrogen in brine, as well. The mole fraction of gas dissolved at the actual conditions is very nearly proportional to the actual partial pressure of the gas (Henry's Law). Therefore, the mole fraction of gas dissolved at the prevailing pressure is

$$
x=X_{\text {dis }} P_{g} \quad \text { where }\left[P_{g}\right]=\text { atm }
$$

Reference 7 cites some slight variation in this proportionality, but the variation is sufficiently small to ignore here. Equations (25) and (26) can be substituted into Eqn. (24) to calculate the variation in the mass of gas dissolved in the brine at the actual pressure.

The solubility relations presented above refer to equilibrium conditions. Like the transient thermal response discussed in Section 4.2, an accurate determination of the quantity of gas dissolved in the brine at any time requires a consideration of the mass-transfer dynamics. The present quasi-steady analysis cannot account for transient effects: a choice must be made to either omit the solubility effects altogether (appropriate for very sluggish mass-transfer dynamics) or include the solubility at the equilibrium values (appropriate for very rapidly equilibrating dynamics).

Mass-transfer dynamics are examined in Appendix A. It is demonstrated there that changes in the quantity of gas dissolved in a quiescent liquid occur very slowly for nitrogen, because of its low mass-diffusion coefficient. Furthermore, the measured variations in temperature and pressure during the GGE program tended to be erratic (non-uniform, certainly, but roughly alternately increasing and decreasing relatively rapidly). Such oscillations tend to limit the penetration of any transported quantity. This effect is similar to the approximately constant soil temperature that persists just a few millimeters below the earth's surface despite significant diurnal variation in the surface temperature. As it turns out, inclusion of the solubility contributions in the calculation program even at the equilibrium values yields results that are virtually indistinguishable from those for which solubility effects were omitted, indicating that the effect is second order, at best, insofar as compensation for the variable external effects are concerned. Solubility effects may be significant if there were ample quantities of other, very soluble, gases, such as $\mathrm{CO}_{2}$ or $\mathrm{H}_{2} \mathrm{~S}$, and the long-term effects of solubility on the WIPP depository performance may well be important. These issues were not considered in the present work.

Although unimportant for this situation, the solubility term is included in the remaining development in this report for completeness.

\subsection{Gas-Generation-Rate Specification}

\subsubsection{Increase in Headspace-Gas Mass by Gas Generation}

The gas-generation term in Eqn. (12) can be solved for explicitly to give 


$$
\Delta N_{g e n}=\frac{P-P_{v}}{P_{0}-P_{v_{0}}}\left(\frac{Z_{0}}{Z}\right) N_{0}\left(\frac{V_{H S}}{V_{H S}}\right)\left(\frac{T_{g_{0}}}{T_{g}}\right)-\left(N_{0}+\Delta N_{D}+\Delta N_{\text {sol }}\right)
$$

This equation then gives the quantity of gas generated between the present calculation point and the reference point once the thermodynamic parameters have been specified. However, the first objective of this work was to demonstrate the validity of the data by examining the "blank", experiment-control test containers (Test Containers No. 152 and 153) for which there could be no gas generation. To do this an alternate form for gas generation is more useful, as developed below.

For the blank test containers, it would be expected that the pressure would be constant after the effects of temperature and headspace-gas-mass variation have been properly nullified. Examination of the two non-waste-bearing containers also serves to find out if the measured pressure, with all of its observed variation, could have been adequately predicted by the present analytical methodology. For this reason, Eqn. (12) is re-written ignoring gas generation, i.e., variations in the headspace-gas mass are assumed to result only from non-gas-generation phenomena. The pressure computed with the specific presumption of no gas generation is designated as simply the "adjusted" pressure, i.e.,

$$
P_{a d j}-P_{v_{0}}=\left(P-P_{v}\right)\left(\frac{Z_{0}}{Z}\right)\left(\frac{N_{0}}{N_{0}+\Delta N_{D}+\Delta N_{s o l}}\right)\left(\frac{V_{H S}}{V_{H S}}\right)\left(\frac{T_{g_{0}}}{T_{g}}\right)
$$

A match of the adjusted pressure at all points to the reference pressure validates the data by demonstrating that the seemingly erratic variation in the measured pressure was, in fact, the expected thermodynamic consequences of temperature and headspace-gas-mass variation. This can be seen clearly by comparing equations (12) and (28).

An adjusted pressure per Eqn. (28) can be computed for all of the test containers, of course, including those bearing potentially gas-generating waste. The motive is that since the adjusted pressure accounts for all of the thermodynamic phenomena that are occurring except gas generation, any non-constant behavior would identify the occurrence of gas generation. Having chosen to compute $P_{\text {adj }}$, as defined by Eqn. (28), it can be used to specify the mass of gases generated as an alternate to Eqn. (27). Dividing Eqn. (28) by Eqn. (12) eliminates the thermodynamic terms, including the measured pressure at the given point, giving

$$
\frac{P_{a d j}-P_{v_{0}}}{P_{0}-P_{v_{0}}}=\frac{N_{0}+\Delta N_{D}+\Delta N_{\text {sol }}+\Delta N_{g e n}}{N_{0}+\Delta N_{D}+\Delta N_{\text {sol }}}
$$

This can be rearranged to give the quantity of gas generated explicitly as

$$
\Delta N_{g e n}=\frac{P_{a d j}-P_{0}}{P_{0}-P_{v_{0}}}\left(N_{0}+\Delta N_{D}+\Delta N_{s o l}\right)
$$


Before concluding the discussion of headspace-gas mass, two other useful representations of the generated-gas mass are developed in the following two sections.

\subsubsection{Mole Fraction of Generated Gases}

Measurements of headspace-gas composition for low concentrations are usually reported as volume fractions of each specie, e.g., as parts per million by volume, $\mathrm{ppm} / \mathrm{V}$. The mole fraction is equivalent to a volume fraction for an ideal gas. In the present case, the increase in the mole fraction of the generated gas, $\Delta X_{\text {gen }}$, at any time relative to that value at the reference point is

$$
\Delta X_{g e n}=\frac{\Delta N_{g e n}}{N}=\frac{\Delta N_{g e n}}{N_{0}+\Delta N_{D}+\Delta N_{\text {sol }}+\Delta N_{g e n}}
$$

Substituting Eqn. (29) into this equation gives

$$
\Delta X_{g e n}=\frac{P_{a d j}-P_{0}}{P_{a d j}-P_{v_{0}}}
$$

Equation (32) shows the usefulness of $P_{\text {adj }}$ in identifying and quantifying gas generation, i.e., $\mathrm{P}_{\mathrm{adj}}-\mathrm{P}_{0}$ non-zero indicates gas generation.

The increased mole fraction of gas generated between any two points, e.g., Point $a$ and Point $b$, is equal to the mole fraction increase at a Point $b$ vis-a-vis the reference value minus the molefraction increase at Point $a$ relative to the same reference value, as demonstrated below:

$$
\begin{aligned}
\delta X_{g e n}=X_{g e n-b}-X_{g e n-a} & =X_{g e n-b}-X_{g e n-0}+X_{g e n-0}-X_{g e n-a} \\
& =\Delta X_{g e n-b}+\left(-\Delta X_{g e n-a}\right) \\
& =\Delta X_{g e n-b}-\Delta X_{g e n-a}
\end{aligned}
$$

The term $\delta X_{\text {gen }}$ would be positive for positive gas generation, and negative for negative gasgeneration. Although negative gas generation, as such, is purely hypothetical, it would be analogous to absorption or dissolution of gas into the brine.

\subsubsection{Rates of Gas Generation}

The rate of gas generation is also typically of interest. This is simply the slope of the gasgeneration rate, and can be readily inferred from a plot of $\Delta X_{\text {gen }}$, per Eqn. (33). The gasgeneration rate should be calculated as an average over a sufficiently large time interval, $\delta t$, so that point-to-point variation in the gas-generation data is adequately filtered. This time interval can be related to time intervals with respect to the reference point by a development analogous to that for Eqn. (33), giving

$$
\delta t=\Delta t_{b}-\Delta t_{a}
$$


The average rate of change of the mole fraction of generated gas in the headspace over the time interval is, therefore,

$$
\dot{X}_{g e n}=\frac{\delta X_{g e n}}{\delta t}=\frac{\Delta X_{g_{e n}}-\Delta X_{g_{e n}}}{\Delta t_{b}-\Delta t_{a}}
$$

This can be evaluated from Eqn. (32).

\subsection{Headspace Volume Considerations}

The volume of the test container headspace, $V_{\mathrm{HS}}$, will be shown to play a very important role in the solution. Its value is not as obvious as it may first appear, as demonstrated in the development in this section.

\subsection{Headspace Volume Variation}

Consistent with the deviation-from-a-reference-point approach being used in this analysis, the headspace volume at some given condition is related to the headspace volume at the reference conditions (zero subscript) by

$$
V_{H S}=V_{H S_{0}}+\Delta V_{H S}
$$

The headspace volume is the volume remaining in the container (subscript $c$ ) after the volume occupied by the brine/waste ("liquid", subscript $l$ ) has been subtracted:

$$
V_{H S}=V_{C}-V_{l}
$$

Differentiating Eqn. (37) and expressing the differential over a small but finite interval gives the increase in headspace volume as directly related to the increases in volumes of the test container and the brine/waste:

$$
\delta V_{H S}=\delta V_{C}-\delta V_{l}
$$

The test container undergoes expansion and contraction as the temperature and pressure change, as do the volumes of brine and each waste constituent. These effects are impressed on the headspace-gas volume, and act in opposite directions. For example, when the temperature rises the test container expands and the internal volume increases slightly, acting to increase the headspace volume. But the simultaneous thermal expansion of the brine/waste acts to decrease the headspace volume. Development of these two competing effects are presented in the following two sections. These seemingly minute effects, induced by very small temperature and pressure changes, will be found to have the most significant consequences for the pressure behavior. 


\subsubsection{Test-Container Expansion/Contraction}

At conditions different from those at the reference conditions the test-container volume is

$$
V_{C}=V_{C_{0}}+\Delta V_{C}
$$

The test container volume depends on its temperature and the differential pressure loading across its boundaries. The latter effect results from variation in the vessel strain caused by changes in the internal pressure relative to the nearly constant glovebox pressure. However, given the extreme stoutness and strength of the containers, the vessel-strain variations can be quickly shown to be fully negligible. Therefore, only the expansion/contraction from thermal effects needs to be considered.

Let the container internal free volume at some known temperature be $V_{\mathrm{C}}$, and let $\alpha$ denote the coefficient of linear thermal expansion of the material of construction. The volume of a container of any shape is proportional to a linear dimension cubed. Invoking this relationship, it can be easily shown that, regardless of the geometric shape of the container, a uniform, infinitesimal temperature change of amount $d T$ from the given state causes a corresponding volume change, $d V_{C}$, of amount

$$
d V_{C}=3 \alpha V_{C} d T
$$

This form can be used directly for small temperature changes, $\delta T$, to give $\delta V_{C}$. Since $\alpha$, itself, frequently is temperature dependent, the average value of $\alpha$ over the given temperature interval is taken for larger temperature changes. Upon integrating Eqn. (40), the test-container volume increase is found to be

$$
\delta V_{C}=V_{C_{0}}\left(e^{3 \bar{\alpha} \delta T_{R T D}}-1\right)
$$

If necessary, this expression can be adapted to the case where the temperature change over the container boundaries is non-uniform by integrating Eqn. (41) over the entire vessel. However, as is discussed later concerning the thermal model, the assumption of uniform temperatures in the vessel barrel at the temperature of the RTD immersed in the brine/waste is quite adequate, allowing Eqn. (41), written relative to the reference point, to be used directly in Eqn. (39).

The free volume of the empty test containers was calculated from dimensional data shown on the design drawings. The volume was calculated to be $7272 \mathrm{ml}$ at the nominal temperature that prevailed during manufacture, assumed to be normal room conditions. Any error in determining the vessel's reference volume has only a small effect on the pressure calculation. Since the experiments were carried out at very near room temperature, the reference volume was therefore simply taken to be $7272 \mathrm{ml}$ at whatever particular reference conditions were selected. 


\subsubsection{Expansion/Contraction in the Brine and Waste}

Volumetric changes in the brine/waste result from both temperature and pressure changes, just as was the case for the test container. An argument similar to that made for the vessel strain was made for the brine/waste as well, viz., that pressure effects are negligible - the brine/waste is assumed to be fully incompressible. This, however, may not actually be true. The brine volume can certainly be shown to be negligibly affected by modest pressure changes, but the response of the waste materials themselves, is less certain. Waste materials may include some relatively compliant constituents, such as rubber, that have relatively low moduli of elasticity. Or, worse still, there may be some trapped gas bubbles among the waste, particularly among the debristypes of waste. Since there was no way to accurately assess this, only the thermally induced expansion/contraction of the brine/waste was accounted for in the methodology.

Defining a volumetric coefficient of thermal expansion for the brine/waste combination as $\beta$, the change in volume of the brine/waste due to thermal expansion is, by definition,

$$
d V_{l}=\beta V_{l} d T_{l}
$$

Similar to the test container thermal expansion, where temperature changes from the reference value are small, this form can be used directly to compute the brine/waste volume change (increase), $\delta V_{l}$. For larger temperature intervals, the change should be computed by integrating Eqn. (42) over the interval and using the average value of $\beta$ over the interval, resulting in

$$
\delta V_{l}=V_{l_{0}}\left(e^{\bar{\beta} \delta T_{R T D}}-1\right)
$$

The similarity between Equations (41) and (43) is apparent. However, it should be appreciated that the coefficient of linear expansion for the container, $\alpha$, pertained to a unique, solid, engineering material and can be readily found in the literature. In the present case, $\beta$ refers to the volumetric expansion of a composite concoction of brine and waste materials. There are no tabulations for this parameter; its value must be determined experimentally, as is discussed later in Sec. 10.1.

Writing Equations (38), (41), and (43) relative to the reference point and substituting these into Eqn. (36) gives the headspace volume at the current point as

$$
V_{H S}=V_{H S_{0}}+\Delta V_{H S}=V_{C_{0}} e^{3 \bar{\alpha} \Delta T_{R T D}}-V_{l_{0}} e^{\bar{\beta} \Delta T_{R T D}}
$$

\subsection{Headspace Volume Determination}

The headspace volume at the reference conditions, $V_{\mathrm{HS}-0}$, was not known. This warrants an explanation. The initial brine/waste volume was not measured prior to loading the test containers, 
so the headspace volume could not simply be determined by subtracting the brine/waste volume from the container free volume via Eqn. (37). Although the masses of waste and brine added to each container were carefully recorded, the densities of the waste materials were not known exactly, and certainly not when submerged in the brine and at operating conditions. The headspace volume was found by gas-expansion measurements, as described in the next section.

\subsubsection{Headspace-Gas Expansion Measurements}

The GGE project determined the headspace volume indirectly by carefully measuring the pressure both before and after an expansion of the headspace gas by appending to the headspace a very small, accurately known volume used to extract a headspace-gas sample. Figure 11 depicts the experimental configuration for determining the headspace volume.

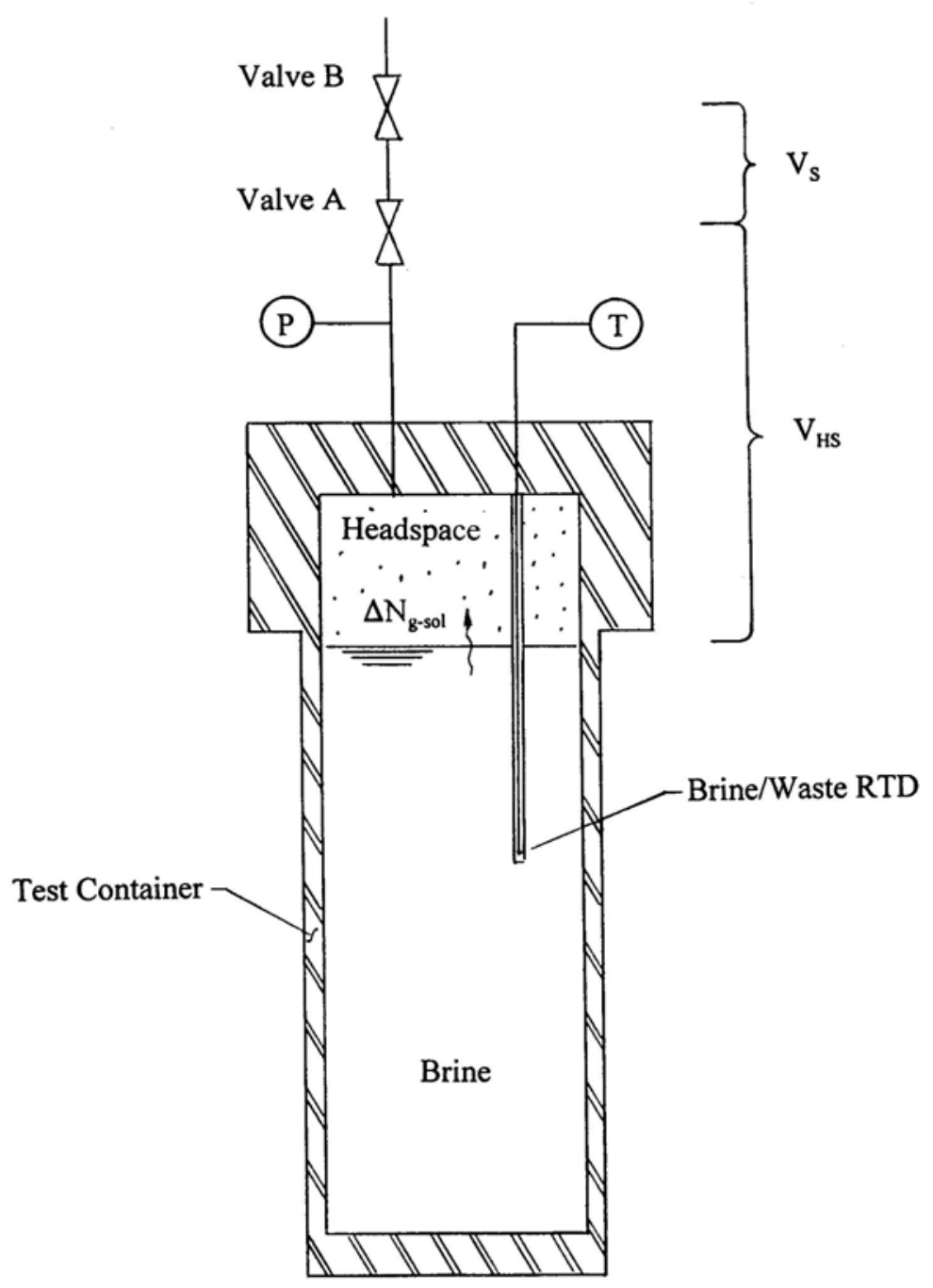

Fig. 11. Depiction of the Headspace Volume Measurement

The mass of gas in the headspace after the gas expansion would be affected if there were a change in the amount of gas dissolved in the brine, and this would influence the gas pressure. 
Solubility effects can be accounted for by recognizing that the total mass of gas within the test container (i.e., the sum of gas in the headspace plus that dissolved in the brine) is constant during the short time span of the sampling event, during which there would be negligible gas generation. Therefore, the mass of gas within the headspace after the expansion must equal that present prior to the expansion plus the change that occurred only as a result of solubility variation. Letting subscripts 1 and 2, respectively, represent the headspace conditions before and after the expansion of the gas across Valve $A$ (Fig. 11) into the known sample volume, $V_{\mathrm{s}}$, the final headspace-gas mass is

$$
N_{2}=N_{1}+\delta N_{\text {sol }}
$$

During the brief time required for the expansion, there are no appreciable gases generated. The gas volume after the expansion is just $V_{\mathrm{HS}}+V_{\mathrm{S}}$. Invoking Eqn. (6) with Eqn. (2), as written for the conditions both before and after the expansion, and combining the equations with Eqn. (45) gives the headspace volume deduced from the pressure measurements as

$$
V_{H S}=\frac{V_{s} \frac{P_{2}-P_{v_{2}}}{z_{2} T_{g_{2}}}-R_{U} \delta N_{s o l}}{\frac{P_{1}-P_{v_{1}}}{z_{1} T_{g_{1}}}-\frac{P_{2}-P_{v_{2}}}{z_{2} T_{g_{2}}}}
$$

Analogous to Eqn. (24), solubility variation over the expansion would cause an equilibrium headspace-gas-mass change of amount

$$
\delta N_{\text {sol }}=N_{l}\left(\frac{x_{1}}{1-x_{1}}-\frac{x_{2}}{1-x_{2}}\right)
$$

Equation (47) can be substituted into Eqn. (46). However, as was noted in Section 5.2.3, the slow dynamics of the gas transport into and out of the brine cause the actual mass change over the short time frame during which the sampling operation occurs to be far less than this equilibrium quantity, so the term in Eqn. (46) containing $\delta N_{\text {sol }}$ can be safely omitted.

The quantities $P_{1}$ and $P_{2}$ in Eqn. (46) are the pressures measured before and after the gas expansion, respectively. $P_{\mathrm{v} 1}$ and $P_{\mathrm{v} 2}$ and the temperatures $T_{g 1}$ and $T_{g 2}$ must be deduced from the brine and glovebox temperature measurements. Normally, this expansion is done slowly and isothermally, allowing the temperatures to remain constant. The sample volume, $V_{\mathrm{s}}$, used in the GGE was determined in a similar way by expanding a gas into a calibrated volume, finding a volume of $3.05 \mathrm{ml}(8)$. 


\subsubsection{Adjustment of the Headspace Volume Measurements}

The headspace volume was measured as described in the preceding section each time a headspace-gas sample was drawn. Typically 5 to 8 such sampling events had occurred for each test container before gas sampling was suspended rather early in the experiment program. Each of these sampling measurements provides a calculated value for the headspace volume at the conditions for which the measurement was made. Per the development cited earlier, Eqns. (36) through (44), the headspace volume depends on the temperatures of the brine/waste and the test container. So unless these temperatures were identical for all sampling events, the headspace volume measurements will be slightly different. To compare these measurements, the measured volumes need to first be adjusted to common conditions. Consistent with the present development where all measurements are corrected back to a specific reference, the headspace volume measurements were all adjusted to the selected reference conditions.

Any difference in the headspace-gas mass between the time when the sampling occurred and the selected reference point (plus possibly some minor difference in the compressibility factor, $z$, between the two points), will effect the pressure, but not the headspace volume. So, only the $\Delta V_{\mathrm{C}}$ and $\Delta V_{1}$ terms need to be considered, and the relation in Eqn. (36) still holds. Eqn. (44) gives the headspace volume at the sampling point in terms of the unknown volume, $V_{1-0}$. Let $V_{\mathrm{HS}-\mathrm{j}}$ denote the raw headspace volume determined for the $j$ th sample as calculated from Eqn. (46). The adjustment of that headspace volume to the corresponding volume that would be found at the reference conditions is as follows. From Eqn. (44), replace $V_{\mathrm{l}-0}$ by it equivalent,

$$
V_{I_{0}}=V_{C_{0}}-V_{H S_{0}}
$$

Then substitute $V_{\mathrm{HS}}$ as found from Eqn. (44) into

$$
V_{H S_{0_{j}}}=V_{H S_{j}}-\Delta V_{H S_{j}}
$$

This yields

$$
V_{H S_{0_{j}}}=\frac{V_{H S_{j}}+V_{C_{0}}\left(e^{\bar{\beta} \Delta T_{R T D_{j}}}-e^{3 \bar{\alpha} \Delta T_{R T D_{j}}}\right)}{e^{\bar{\beta} \Delta T_{R T D_{j}}}}
$$

Equation (50) can be evaluated immediately when the coefficient $\beta$ is known. For the case of pure brine, a separate experiment was performed to determine the value of $\beta$. The experiments are discussed in Appendix B. In the general brine/waste case, however, $\beta$ is unknown. Its determination is discussed in Section 10.1. It will be shown in Section 10.1 that $\beta$, itself, depends on the headspace volume at the reference conditions. Therefore, a simultaneous solution with Eqn. (50) will be found necessary to determine the adjusted headspace volume, $\mathrm{V}_{\mathrm{HS}-0 \mathrm{j}}$, for each measurement. 
Ideally, all of the headspace volume measurements should yield precisely the same value after having been adjusted to common conditions (such as at the reference point). However, the individual headspace volume calculations are based on field measurements, so some variation among the measurements must be expected, and this variation carries over to the adjusted values. The $j$ subscript on the adjusted headspace volume in Eqn. (50) emphasizes that this result pertains specifically to the $j^{\text {th }}$ field measurement. An arithmetic average over all of the $n$ adjusted volume measurements will yield the best estimate of the true mean volume at the reference conditions:

$$
V_{H S_{0}}=\frac{1}{n} \sum_{j=1}^{n} V_{H S_{0_{j}}}
$$

The headspace volumes determined from Eqn. (50) are listed in Table 2, along with their corresponding reference-point temperatures. The headspace volume for each test container allows the corresponding brine/waste volume, $V_{1-0}$, to be found from Eqn. (48). Then $V_{\mathrm{HS}}$ can be calculated from Eqn. (44), allowing the ratio $V_{\mathrm{HS}-0} / V_{\mathrm{HS}}$ to be evaluated.

Table 2. Best Estimate Headspace Volumes at the Reference Conditions

\begin{tabular}{|l|c|c|}
\hline $\begin{array}{c}\text { Test } \\
\text { Container } \\
\text { No. }\end{array}$ & $\begin{array}{c}\text { Reference Temperature } \\
(\mathrm{C})\end{array}$ & $\begin{array}{c}\text { Headspace Volume } \\
(\mathrm{ml})\end{array}$ \\
\hline \hline TC-151 & 31.1 & 631 \\
\hline TC-152 & 31.0 & 733 \\
\hline TC-153 & 31.0 & 727 \\
\hline TC-160 & 31.1 & 1080 \\
\hline TC-161 & 31.3 & 905 \\
\hline TC-161e ${ }^{\text {a }}$ & 31.2 & 905 \\
\hline TC-162 & 30.7 & 910 \\
\hline TC-163 & 31.0 & 945 \\
\hline TC-164 & 30.8 & 923 \\
\hline TC-165 & 30.7 & 807 \\
\hline TC-166 & 31.1 & 765 \\
\hline TC-167 & 31.3 & 911 \\
\hline TC-168 & 31.3 & 912 \\
\hline TC-169 & 30.2 & 849 \\
\hline TC-170 & 31.1 & 743 \\
\hline
\end{tabular}

a. TC-161e uses empirical calibration constants for TC-161. 


\subsection{Headspace-Gas Compressibility}

For a truly thermally perfect gas, $z$ implicitly has a constant value equal to unity under all conditions. However, for real gases the compressibility factor, itself, is dependent on temperature and pressure; thus, the apparent simplicity of writing a state equation like Eqn. (2) that includes such a factor is misleading. The compressibility factor can take on values greater or less than unity, depending on specific conditions. The usefulness of specifying the state equation in terms of $z$ lies in the fact that for many applications only the initial and end states are needed. For other processes over a small range, it may be acceptable to approximate $z$ as a constant. In both of these cases, the compressibility factor can be read directly from published tables or plots.

Finding the ratio $z_{0} / z$ is, in principle, straightforward. The factors can be read directly for a given temperature and pressure from charts like that shown in Fig. 12 for nitrogen, the predominant gas in the GGE containers. However, for the small changes in temperature and pressure that occurred in the GGE, the scale of the compressibility plot is much too small to determine the ratio accurately. Furthermore, for analysis usage, it is preferable to have a mathematical representation so that $z$ can be programmed and calculated. This can be done by using one of the many equations of state that have been proposed to describe the behavior of real gases.

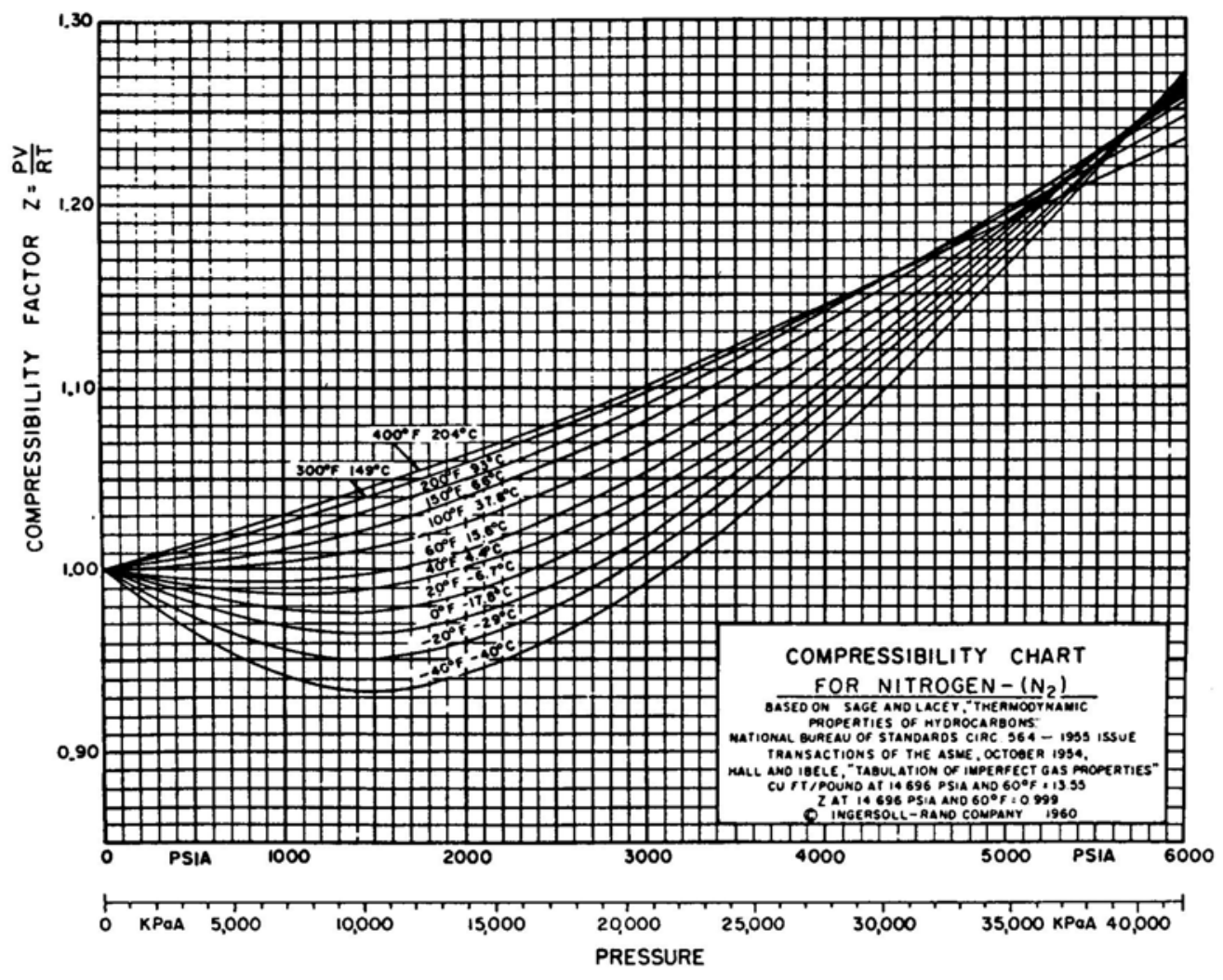

Fig. 12. Compressibility Factor for Nitrogen Gas 
The Beattie-Bridgeman equation of state has been found to produce results in excellent agreement to experimental measurements for many gases over a wide pressure range, including those of interest to the GGE. This equation of state was selected for this analysis. The relation is

$$
P_{g}=\frac{\left[R_{u} T_{g}(1-e)\left(v_{g}+B\right)-A\right]}{v_{g}^{2}}
$$

where $R_{\mathrm{U}}$ is the universal gas constant and $v_{g}$ is the mole-specific volume (2). The constants $A$, $B$, and $e$ are defined in terms of various constants specific to a given gas and other thermodynamic parameters:

$$
\begin{gathered}
A=A_{0}\left(1-a / v_{g}\right) \\
B=B_{0}\left(1-b / v_{g}\right) \\
e=\frac{c}{v_{g} T_{g}^{3}}
\end{gathered}
$$

These are units-dependent equations, formulated for units of

$$
\begin{aligned}
& {\left[P_{g}\right]=\text { atm }} \\
& {\left[T_{g}\right]=\mathrm{K}} \\
& {\left[v_{g}\right]=\text { liter/g-mole }}
\end{aligned}
$$

In these units the universal gas constant, $R_{\mathrm{U}}$, is $0.08206 \mathrm{~atm}-\mathrm{liter} / \mathrm{g}-\mathrm{mole} / \mathrm{K}$. For nitrogen, the specific constants needed are (2)

$$
\begin{gathered}
A_{0}=1.345 \\
a=0.02617 \\
B_{0}=0.05046 \\
b=-6.910 E-03 \\
c=42.00 E+03
\end{gathered}
$$

The Beattie-Bridgeman equation, however, is awkward to use because it is transcendental. An iterative solution for specified pressure and temperature was performed by evaluating the righthand side of Eqn. (52) with Eqn. (53) and Eqn. (54), using assumed values of the mole-specific volume, $v$, until a match to the left-hand side was achieved. An algorithm for rapidly performing this trial solution was developed and included in the computer-calculation program discussed later.

By combining the result with the gas law, Eqn.(2), an analytical expression for the compressibility factor becomes 


$$
Z=\frac{(1-e)\left(v_{g}+B\right)-\frac{A}{R_{U} T_{g}}}{v_{g}}
$$

Evaluating Eqn. (55) at the reference conditions and at the conditions of each data point under consideration allows determination of the ratio $z / z_{0}$. Compressibility factors calculated with this equation for nitrogen show very good agreement to the experimental data shown in Fig. 12 up to about 3000 psia, well in excess of the pressure range applicable to the GGE.

\subsection{Headspace-Gas Temperatures}

The final term needed for the evaluation of the adjusted pressure, Eqn. (28), and the gas generation rate, Eqn. (35), is the ratio of the temperature in the headspace gas at the current point to the headspace-gas temperature at the reference point. This ratio is developed in this section.

\subsection{Equilibrium Temperatures}

The temperatures in Eqn. (2) refer, of course, to the that of the headspace gas. However, not only is the temperature in the headspace non-uniform, there was no instrumentation in the GGE experiments to measure any headspace temperature. The test-container temperature was measured by an RTD placed in a thermowell immersed in the brine, as shown in Fig. 3. Examination of the vessel geometry and the typical temperature traces for test container and glovebox RTD's shown in Fig. 8 suggest that the temperature in the headspace gas is not necessarily accurately represented by the thermowell-RTD measurement: the top region of the vessel is surrounded by the cooler glovebox environment that will certainly cause the headspace gas to be somewhat cooler than the brine/waste. Thermal conditions within the test container were therefore modeled to better estimate the true headspace-gas temperature from the limited temperature data.

Two steady-state, finite-difference thermal models were developed. The first was a twodimensional, axi-symmetric model whose node structure is shown in Fig. 13. The model represented the geometry of the actual vessel quite well, requiring only some small adjustments to smear certain features in the top head and miscellaneous fittings such as the brine-filler tube, etc. to an approximate equivalent in rotationally symmetric geometry. When suitable relations for the thermal conductance in the various regions and at the interfaces were applied, the model could be executed with varying electric power at the heating band location until the calculated temperature at the thermowell location matched the actual RTD measurement. A specified heat source was imposed on nodes $(\mathrm{i}, \mathrm{k})=(10,3),(10,4)$, and $(10,5)$ in the discretized model shown in Fig. 13, since only the lowest heating band was energized. The heater power was proportioned among these nodes in relation to the fractional coverage by the heater band on each node. The temperature distribution throughout the entire test vessel would then be known, including, in particular, the average temperature in the headspace gas. 


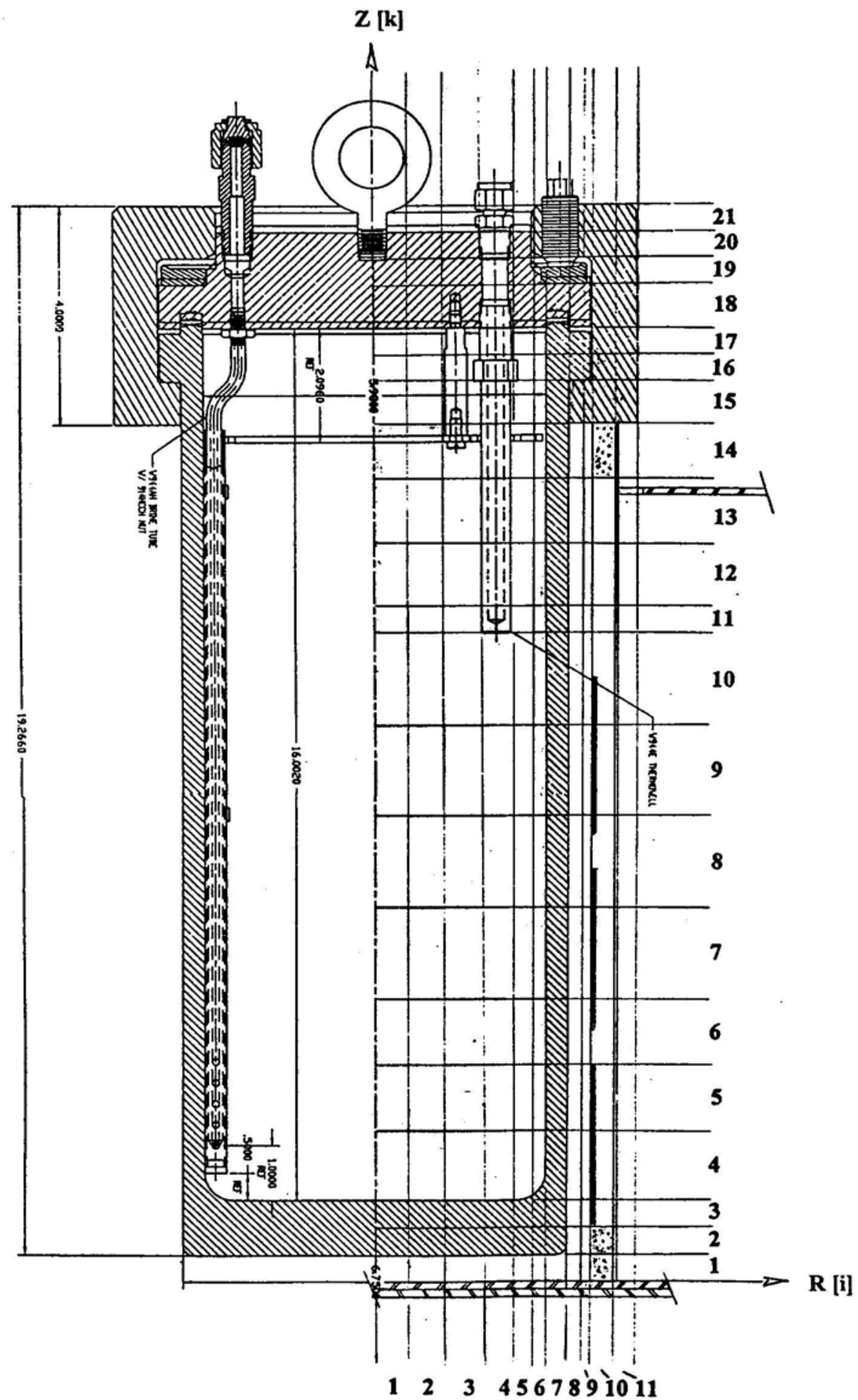

Fig. 13. Discretization for the Two-Dimensional Thermal Model 
Finding the match of the calculated RTD-node temperature to the measured value is simpler than it may appear if the heat transfer in the brine and headspace results only from conduction. First, a normalized, non-dimensional temperature is defined with the bottom wall of the test vessel set to temperature unity, and the glovebox environment set to temperature zero. Execution of the thermal model on this basis and with an assumed value for the heater power will yield a corresponding temperature distribution throughout the model. Because of the linearity of the conduction equation, all of the temperatures may be scaled to find the desired values by using a scale factor equal to the ratio of the target RTD node temperature to the temperature calculated at the node. Only when there are temperature-dependent properties and/or inter-node conductances, or non-linear convective heat-transfer correlations will a scaling approach like this be compromised, but even in that case, the scale factor can be used to guide the second guess for the heater input that will usually yield very good results.

Natural convection in the brine picks up heat from the lower end of the test containers (where the heater band is located) and transports the heat to the brine surface. Upon giving up the heat, the brine is somewhat cooled, increasing its density and causing it to sink back to the bottom of the test container to begin another cycle. This phenomenon creates vertical, recirculating cells of fluid movement. The geometric structure of these cells might range from a single core of warm fluid surrounded by a counter-flowing "jacket" of the cooler fluid (or vice versa) to, more probably, multiple, parallel micro cells of warmed and cooled counter-flowing fluids. A full twodimensional treatment requires that the convection in this region, and within the headspace region, be modeled two dimensionally. This is a difficult calculation to do accurately, and, in any event, a full two-dimensional analysis will be quite time-consuming to run (the archived data examined for each of the 14 test container over the initial 3.25 years of testing consisted of over 26,000 data points, each point of which requires the thermal model to be executed). Some simplification was therefore investigated to ease this computational burden. This simplification is discussed below.

Gross radial temperature variation results only from heat losses from the sides of the test container. Since the body of the test container is surrounded by a thick gap of confined argon gas, which has a relatively low thermal-conductivity coefficient, radial heat losses in that region should be modest. A much simpler, one-dimensional (axial) thermal model was therefore subsequently developed.

The one-dimensional model is depicted in Fig. 14. This is a simple series heat-transfer model, based on the presumption of only second-order temperature variation radially over the body of the test container. Some adjustments, described shortly, were made to account for twodimensional effects in the upper region. In this model, all heat is transferred from the lower region of the brine/waste to the container head and then out to the glovebox environment through the top of the vessel head. The mathematic representation is quite simple. The heat flow rate, $q$, is determined from the series resistances, the inverse of the conductances, $h$, between the hot and cold temperatures. With reference to Fig. 14, 
$T_{\infty}$

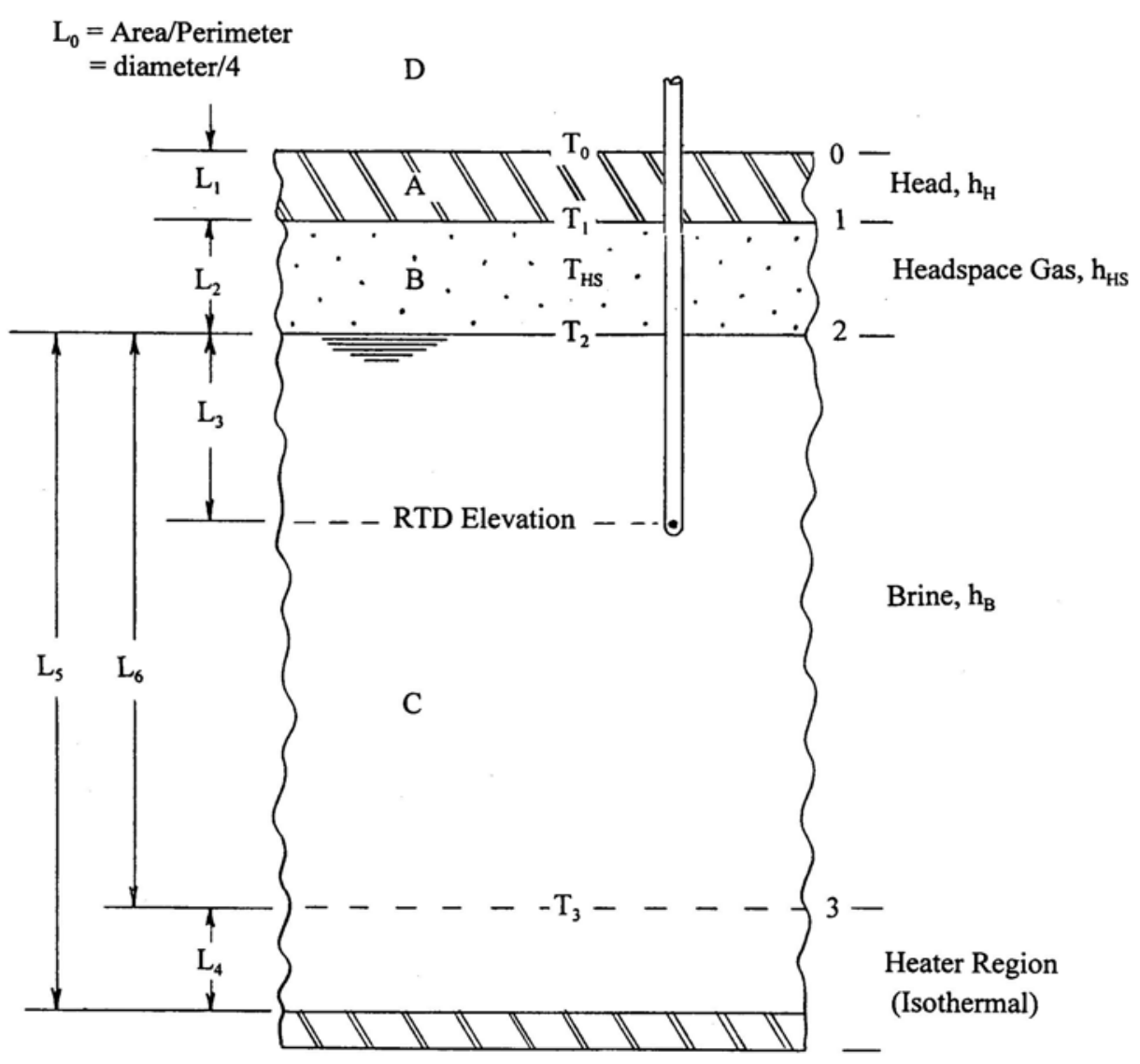

Fig. 14. One-Dimensional Thermal Model

$$
\begin{aligned}
& T_{0}=\frac{q}{h_{\infty}}+T_{\infty} \\
& T_{1}=\frac{q}{h_{H}}+T_{0} \\
& T_{2}=\frac{q}{h_{H S}}+T_{1} \\
& T_{3}=\frac{q}{h_{B}}+T_{2}
\end{aligned}
$$

Summing these equations gives the uniform heat flow rate as

$$
q=\frac{T_{3}-T_{\infty}}{\frac{1}{h_{B}}+\frac{1}{h_{H S}}+\frac{1}{h_{H}}+\frac{1}{h_{\infty}}}
$$


The four conductance parameters ( $h$ 's) can be estimated from various correlations in the literature and are functions of material properties, which are possibly temperature dependent. The five unknown quantities are $q, T_{0}, T_{1}, T_{2}$, and $T_{3}$. The five relations given in Eqn. (56) and (57) are not linearly independent, however, since Eqn. (57) is just the sum of the other four equations. Another relationship is needed. This involves the temperature as measured by the brine RTD, per the argument given below.

The rate of heat transport within the brine/waste by natural convection is much greater than that by thermal conduction. This is borne out by the high Rayleigh Number, indicative of the ratio of these two heat-transport mechanisms, that exists in this region. Since natural convection dominates heat transport in the brine/waste, and since the radial heat transport in this region has been argued above to be relatively low, the bulk temperature in this recirculating region must be nearly isothermal, and thus well approximated by the brine/waste RTD measurement. A sketch of the probably axial temperature distribution in the brine/waste is shown in Fig. 15. It is also noted that in this one-dimensional model the heated and cooled surface areas of the brine/waste are both approximately equal to the test container interior cross-sectional area. Furthermore, the heat transfer at the lower brine region and heat transfer at the upper brine surface both involve higher temperatures underneath the interface of interest, so the heat-transfer coefficients should be comparable. Therefore, the temperature drops in the brine/waste at the lower and upper regions in the brine must be comparable. This argument is equivalent to forcing the bulk brine/waste temperature to be equal to the RTD measurement and at the arithmetic average temperature. With reference to the region and interface numbering shown in Fig. 14, $T_{3}$ (or $T_{2}$ ) can therefore be expressed in terms of the measured RTD temperature, $T_{\mathrm{RTD}}$, from

$$
T_{R T D}=\frac{T_{2}+T_{3}}{2}
$$

Only in the upper head and clamp region do multi-dimensional effects become significant. There, as depicted in Fig. 16, heat from the headspace is transported to the glovebox environment from both the upper and the cylindrical sides of the massive head and clamp assembly. The larger cross-sectional area and outer surface area contribute to a reduced resistance to heat flow from inside to the glovebox interior compared to the one-dimensional model. To account for this, two methods were used to determine an effective conductance, $h_{\mathrm{H}}$, which, when used in the onedimensional model, would yield approximately the same heat transfer rate as would occur in the actual geometry with the appropriate transport properties. 


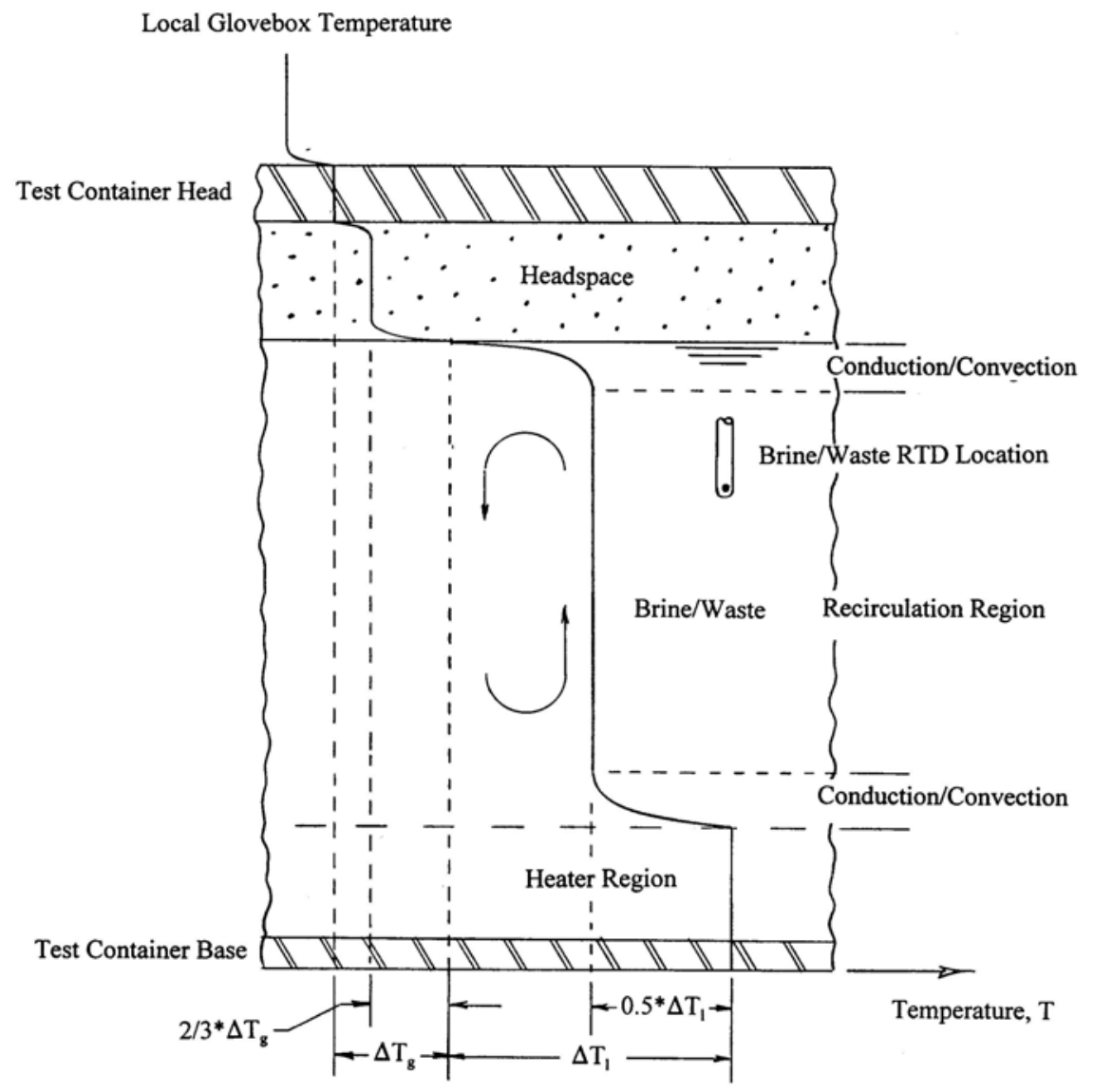

Fig. 15. Hypothesized Axial Temperature Distribution in the Brine/Waste

T.

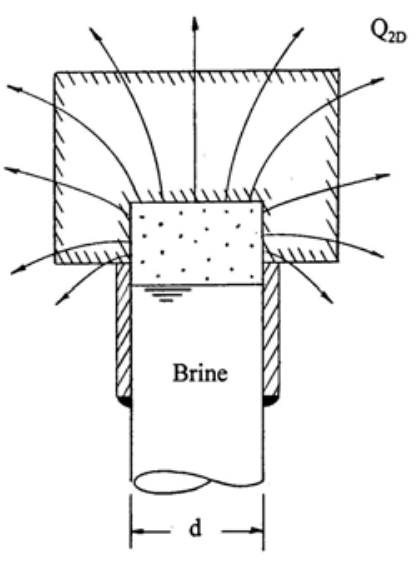

Actual Two-Dimensional Heat Flow
T.

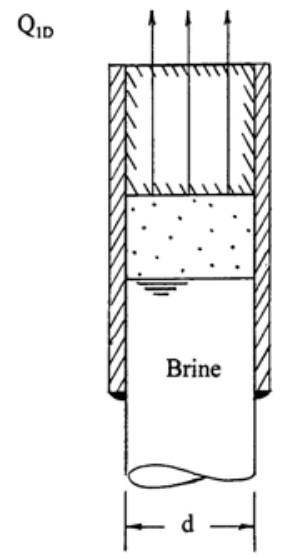

Modeled One-Dimensional Heat Flow

Fig. 16. Two-Dimensional Effects in the Test-Container Head Region 
One method was to model the upper head and clamp regions carefully using the two-dimensional model previously described and impose a constant temperature on the inside surfaces of the vessel head. The heat flow rate to the fixed-temperature environment could then be equated to the heat-flow rate from the one-dimensional model having the same temperature beneath the head to determine an effective conductance value. This effect can be expressed as a multiplier on the normal transport properties of the head material. Instead of a constant factor, the factor varied somewhat depending on the temperature level because of the temperature dependency of the natural convection at the outer surface. These calculations found a typical multiplier factor of about 4.35, but are not exact because of the impressed uniform temperature assumption on the inner surface.

A second method involved computing an effective cross-sectional area for heat transport through the head. The second method requires only a knowledge of the geometry. The inner and outer surface areas of the head were simply averaged, and this value was divided by the onedimensional cross-sectional area. This method found a multiplication factor of about 5.1. This calculation does not accurately account for the contact conduction resistances between head and clamp nor the actual head orientation insofar as surface conductances are concerned. As a result of these comparisons, the heat-transport coefficient for the head and the outer surface in the onedimensional model were simply augmented by a factor of 5.0, and this factor was used consistently in all applications of the one-dimensional model.

A high Rayliegh number was also found to exist within the headspace-gas region. Again, this indicates that heat transport by conduction through the headspace gas is low compared to that transported by natural convection. So, by argument similar to that given above concerning heat transport in the brine/waste, the bulk gas temperature in the headspace must also be nearly uniform. Heat-transfer coefficients should be comparable over all of the boundaries of the headspace. However, the cylindrical surface around the headspace provides an additional coolsurface area for heat transfer that, together with the area provided by the underside of the head, is larger than the hot surface area (the surface of the brine). So although the isothermal assumption in the headspace remains valid, the mean headspace-gas temperature favors the cooler temperature of the underside of the container head closure instead of the average of the temperatures on the underside of the container head and on the brine surface. Ratioing the surface areas bounding the headspace cavity in the two-dimensional and one-dimensional models, the mean temperature is biased toward the temperature on the underside of the head by about 2:1. For example, if the brine surface were at $30^{\circ} \mathrm{C}$ and the underside of the test container head were at $15^{\circ} \mathrm{C}$, the bulk headspace-gas temperature must be approximately $20^{\circ} \mathrm{C}$. Mathematically, this relation is just

$$
T_{H S}=T_{1}+\frac{\left(T_{2}-T_{1}\right)}{3}
$$

Once the various heat-conductance coefficients have been evaluated the interface temperatures in the model, i.e., $T_{0}, T_{1}$, and $T_{2}$, can be quickly found from Eqn. (56)-(58), allowing $T_{\mathrm{HS}}$ to be determined from Eqn. (59). It is noted, however, that three of the four conductance parameters involve natural convection. These are rather sensitive functions of temperature, requiring 
iterative solutions to ensure that they are based on temperatures that are consistent with the final computed temperature distribution. Therefore, while conceptually simple, the actual solution of even the one-dimensional model becomes somewhat tedious. A comprehensive FORTRAN program was written and used. This program evaluated the conductance parameters using an assumed temperature distribution and then iterated the solution until satisfactory convergence was obtained. The entire thermal model program was then embedded as a subroutine in the overall analysis calculation program.

Table 3 lists the thermophysical properties and parameters selected for this application.

Table 3. Thermo-Physical Properties Used in the Thermal Model

\begin{tabular}{|l|c|c|c|c|c|}
\hline & $\begin{array}{c}\text { Temperature } \\
{[\mathrm{C}]}\end{array}$ & $\begin{array}{c}\text { Thermal } \\
\text { Conductivity } \\
{[\mathrm{W} / \mathrm{m} / \mathrm{C}]}\end{array}$ & $\begin{array}{c}\text { Kinematic } \\
\text { Viscosity } \\
{\left[\mathrm{m}^{2} / \mathrm{s}^{2}\right]}\end{array}$ & $\begin{array}{c}\text { Constant-Pressure } \\
\text { Specific Heat } \\
\text { Capacity } \\
{[\mathrm{J} / \mathrm{kg} / \mathrm{C}]}\end{array}$ & $\begin{array}{c}\text { Mass Density } \\
{\left[\mathrm{kg} / \mathrm{m}^{3}\right]}\end{array}$ \\
\hline \hline Glovebox Gas (Argon) & -23.16 & 0.0152 & $1.20 \mathrm{E}-05$ & 520 & 1.630 \\
& 26.84 & 0.0177 & $1.69 \mathrm{E}-05$ & 520 & 1.358 \\
& 126.84 & 0.0223 & $2.84 \mathrm{E}-05$ & 520 & 1.019 \\
\hline Test-Container Head & 25 & 12.6 & -- & -- & - \\
(Hastelloy-C) & & & & & 200.4 \\
\hline Headspace Gas & -23.16 & 0.0222 & $7.78 \mathrm{E}-08$ & 1038 & 167.0 \\
(Nitrogen) & 26.84 & 0.0260 & $1.08 \mathrm{E}-07$ & 1041 & 124.9 \\
& 126.84 & 0.0325 & $1.79 \mathrm{E}-07$ & 1046 & 1234 \\
\hline Brine/Waste (Brine/ & 0 & 0.501 & $1.44 \mathrm{E}-06$ & 3399 & 1222 \\
Water) & 20 & 0.597 & $8.16 \mathrm{E}-07$ & 3393 & 1210 \\
& 40 & 0.628 & $5.36 \mathrm{E}-07$ & 3401 & 3408 \\
\hline
\end{tabular}

As a final note, the thermal model is based on knowledge of the bulk temperature within the glovebox, $T_{\infty}$, in the immediate vicinity of the test container head, i.e., the local glovebox temperature. The test-container heaters hold the brine/waste temperature above that of the ZPPR Cell unless the ZPPR Cell were to be hotter than the heater setpoint, which never occurred. Therefore, the local bulk temperature within the glovebox is always somewhere between the temperature of the brine/waste and that of the ZPPR Cell. This temperature difference was termed the "excess” temperature, i.e., excess over the glovebox RTD measurement.

There was, in fact, no actual measurement of the local glovebox temperature. This is because of the poor choice for placement of the glovebox RTD, which was directly adjacent to the glovebox wall and underneath the subfloor (see position in Fig. 7). This placement causes the instrument to actually measure some intermediate temperature between that of the bulk glovebox interior and the ZPPR cell. To take at least some account for this measurement error, an algorithm, based on some rather crude temperature mapping within the glovebox, was included in the thermal model to estimate the local glovebox temperature, $T_{\infty}$. The algorithm used was a $2^{\text {nd }}$-order polynomial fit to the measurements of the excess temperature versus that measured by the glovebox RTD. The equation fit was generated using the Microsoft EXCEL-97 program. For glovebox RTD 
temperature, $T_{\mathrm{GB}}$, measurements in Celsius, the excess temperature was estimated from the relation

$$
\delta T_{e X}=0.0215 T_{G B}^{2}-1.2975 T_{G B}+21.503
$$

The local glovebox temperature is then just the sum of $T_{\mathrm{GB}}$ and $\delta T_{\mathrm{ex}}$. The temperature-mapping data from which this algorithm was deduced is described in Appendix C.

Equation (59) provides the headspace-gas temperature for each set of RTD and glovebox temperatures once the thermal model has been executed. Calculation of the thermal model at the reference conditions and at each data point enables the desired headspace-gas-temperature ratio, $T_{g} / T_{g 0}$, to be computed.

\subsection{Mechanical Generation of Heat}

It may be noted that the preceding analysis has made no consideration of the thermodynamic effects on temperature in the headspace gas that result from compression or expansion. This is worth further consideration, because to the headspace gas the thermal expansion or contraction of the brine/waste, however small the magnitude of the volume change may be, seems like a piston performing mechanical work (algebraically) on the gas. Conservation of energy considerations from elementary thermodynamics show that this process should affect the gas pressure and temperature, even in the absence of any heat transfer between the gas and its surroundings. Figure 17 depicts this process. 

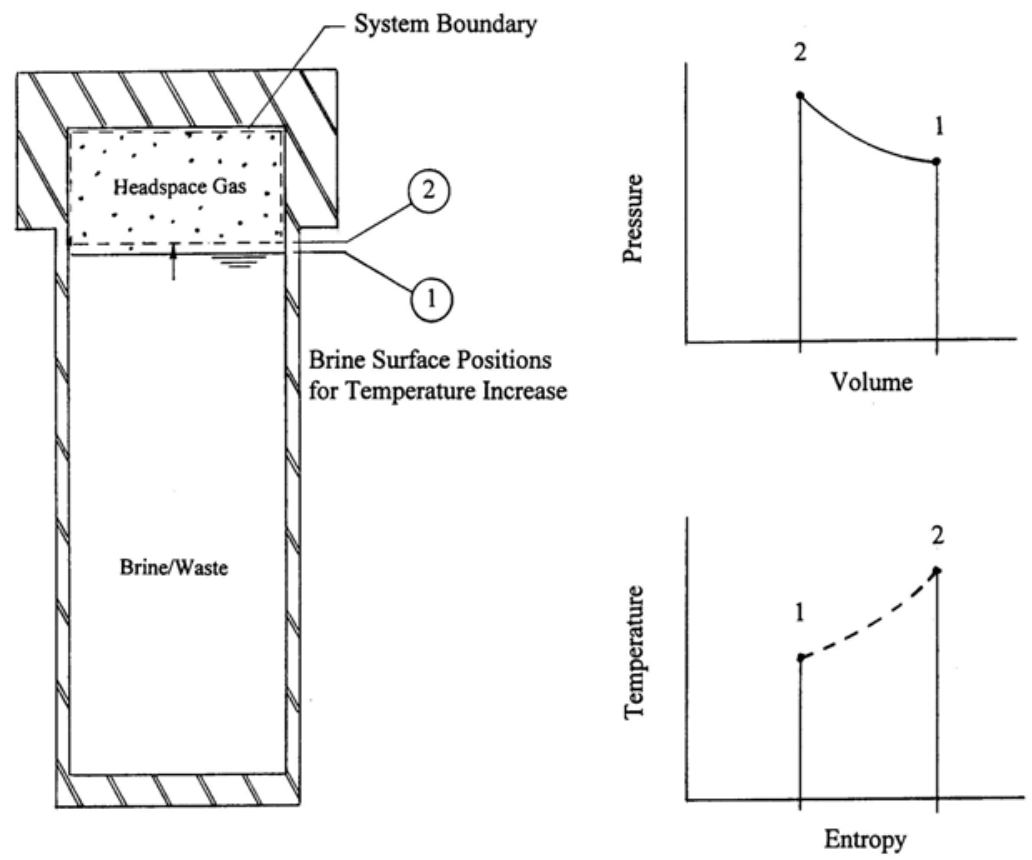

Fig. 17. Mechanical Work of Compression (or Expansion) in the Headspace Gas

This work process would, indeed, cause temperature changes in the gas and be important in a transient treatment of the problem. But the quasi-steady analysis presented here is fully indifferent to the particular process(es) by which the new thermodynamic states are achieved. In other words, the new temperature of the gas will eventually return to precisely that temperature dictated by equilibrium considerations between the gas and its surroundings, no matter what other processes may have taken place.

Even though mechanical work considerations have no role in the present, quasi-steady-state, analysis, a better understanding of the test container's temperature response can be gained by exploring this further. The process considered is a simple compression of the headspace gas by the expanding brine/waste (caused by an increase in the brine/waste temperature). A " 1 st Law", or conservation of energy, statement for the process for the "closed" system (i.e., no mass flows crossing the boundary) shown in Fig. 17 is

$$
d U=d Q-d W
$$

The terms on the right-hand side of this equation are not properties of the thermodynamic state, but, instead, are process dependent. The quantity $W$ is the work performed by the system (the headspace gas, itself, as depicted in Fig. 17), $Q$ is the heat transfer to the system across the system boundary, and $U$ is the internal energy. The change in the internal energy in the gas as the "piston" moves from position 1 to position 2 is

$$
\Delta U=Q_{1-2}-W_{1-2}
$$


The heat transfer, $Q_{1-2}$, must be determined from a thermal analysis as the gas goes from equilibrium state 1 to the new equilibrium state 2 . The work performed by the system (headspace gas) on the piston (brine/waste), $W_{1-2}$, for a reversible (no parasitic losses such as turbulence) moving-boundary process in a closed system is just

$$
W_{1-2}=\int_{1}^{2} P d V
$$

The internal energy of an ideal gas is a function only of its temperature. This fact with the definition of the specific heat capacity at constant volume, $c_{\mathrm{V}}$, relates the change in internal energy over the process to the change in the temperature as

$$
\Delta U=M_{g} c_{V} \Delta T
$$

This equation can also be written in terms of moles, but the form above is more conventional. The term $c_{\mathrm{V}}$ is the average value over the temperature range of interest, and, for nitrogen at room temperature, has a value of about 7.08E-3 atm-litre/g/K.

Combining Equations (62) through (64) gives the temperature increase in the gas as

$$
\Delta T=\frac{1}{M_{g} c_{V}}\left(Q_{1-2}-\int_{1}^{2} P d V\right)
$$

If the headspace gas were perfectly thermally insulated from its surroundings, i.e., insulated from the headspace walls and from the piston (brine/waste), there would be no heat transfer, so $Q_{1-2}$ would be zero. Eqn. (64) indicates that there would nevertheless be a temperature change in the gas as a result of the mechanical work, as was stated at the beginning of this section. Furthermore, since $P$ is positive and $d V$ for the system (gas) is negative for the compression process being considered, the change in the temperature would be positive, i.e., the gas temperature would increase, as expected. An expanding brine/waste scenario implies that the brine temperature is rising, so it would follow that heat is probably also being transferred to the gas, i.e., $Q_{1-2}$ would be positive. This is a rate-limited process, but does act to increase the value of $\Delta T$, and accounts for the increasing entropy depicted in Fig. 17.

In the actual case there will always be heat transfer of whatever magnitude and direction is needed to achieve thermal equilibrium between the gas and its surroundings (the test container head and the brine/waste). Whether the mechanical work causes the gas temperature to momentarily under-shoot, over-shoot, or just equal the new equilibrium value will depend on the values of the specific parameters, such as $c_{\mathrm{V}}, V_{\mathrm{HS}}, \beta$, etc. It will be demonstrated in Section 9.1.7 that the parameters relevant to GGE cause gas temperature changes that result from mechanical work to be non-negligible and to somewhat under-shoot the equilibrium value. This means that the role of mechanical work in GGE is to assist in the rapid attainment of the equilibrium temperatures, shortening the transient period required for heat transfer with the surroundings to 
achieve the new equilibrium state. This adds further confidence to the appropriateness of the quasi-steady analysis presented in this report.

\subsection{Validation of the GGE Data}

To demonstrate both the validity of the GGE data and the utility of the analytic methodology, the example that was discussed in Section 1.3 and shown in Fig. 1 and Fig. 2 is considered once again. The full temperature traces measured for this test container (TC-153) were shown in Fig. 8. At about data point no. 16000 the heater set point on this container was deliberately raised, producing the sharp temperature rise shown (this is the temperature rise that was shown on a greatly expanded time scale in Fig. 1.) Within about $12 \mathrm{~h}$ the RTD temperature within this test container increased $10.2^{\circ} \mathrm{C}$, from $29.6^{\circ} \mathrm{C}$ to $39.8^{\circ} \mathrm{C}$. There was only a small effect on the glovebox temperature from the increased heat load in the glovebox, as seen in the same figure. The pressure within the test container (see Fig. 2 and Fig. 9) increased during this event from 14.615 MPa (2119.7 psia) to 15.663 MPa (2271.7 psia) according to the data records. Some time after reaching the high temperature the heater was then reset (evidently, somewhat lower than the original setting) and the temperature (and pressure) dropped to below their initial levels, as evident in Fig. 1 and Fig. 8. Further manipulation of the heater controller eventually reestablished the original conditions.

Attention here is focused only on the initial rise in the test-container temperature from $29.6^{\circ} \mathrm{C}$ to $39.8^{\circ} \mathrm{C}$. Recall that the earlier consideration of only the direct temperature effect for this case (see Section 1.3) failed badly to predict the actual pressure rise. A viable explanation of the physical phenomena must be able to accurately predict the accompanying pressure rise induced by this simple step in the temperature. This temperature change was much larger than the temperature variations that occurred during the normal GGE operation. If the methodology presented here correctly handles this relatively large-scale event, it can be expected to perform well over all of the data. The calculation below uses the methods described in the preceding sections to predict the pressure rise.

\subsection{Computation at a Specific Point}

For the present example, select the reference point to be those conditions that existed at the initiation of the temperature excursion: test-container RTD temperature of $29.6^{\circ} \mathrm{C}$, glovebox temperature of $20.6^{\circ} \mathrm{C}$, and pressure of $14.615 \mathrm{MPa}(2119.7 \mathrm{psia})$.

\subsubsection{Temperature Ratio}

The local temperature within the glovebox in the vicinity of the test container was estimated by the algorithm in Eqn. (60) to be $3.90^{\circ} \mathrm{C}$ in excess of the temperature measured by the glovebox RTD at the cited reference point. At these conditions, the one-dimensional thermal model estimates a mean headspace-gas temperature of $28.29^{\circ} \mathrm{C}$. The vapor pressure (of water) at this gas temperature is $3.847 \mathrm{kPa}(0.558 \mathrm{psia})$. At the conditions that occur at the peak of the temperature rise, the test-container RTD measured $39.8^{\circ} \mathrm{C}$, the glovebox RTD measured $20.9^{\circ} \mathrm{C}$, and the local temperature excess over the glovebox RTD measurement was estimated to be $3.76^{\circ} \mathrm{C}$. At these conditions the mean headspace-gas temperature was found from the one- 
dimensional thermal model (Section 8.1 ) to be $36.30^{\circ} \mathrm{C}$. The vapor pressure for water at this gas temperature is $6.047 \mathrm{kPa}(0.877 \mathrm{psia})$.

The ratio of temperatures within the headspace was therefore estimated to be

$$
\frac{T_{g}}{T_{g 0}}=\frac{36.30+273.15 K}{28.29+273.15 K}=1.02658
$$

It is recognized that the overall accuracy of the methodology certainly does not warrant six significant digits. These are shown in this section only to highlight the variation.

\subsubsection{Compressibility Ratio}

The Beattie-Bridgeman equation, Equations (52) through (55), with the mean gas temperature and pressure at the reference conditions, gives $z_{0}=1.02382$. The final pressure for this calculation is unknown, so a guess is required to calculate $z=z\left(P, T_{\mathrm{g}}\right)$. After a new predicted pressure has been found, the process can be iterated by successive substitution, using that pressure to find an improved value for $z$, computing an improved pressure, etc. The convergence requires a few iterations, but is straightforward. To expedite this example, simply use the final measured pressure of $15.663 \mathrm{MPa}(2271.7 \mathrm{psia})$ to compute the compressibility factor, giving $z=1.03438$. So the compressibility ratio is found to be

$$
\frac{Z}{z_{0}}=\frac{1.03438}{1.02382}=1.01032
$$

\subsubsection{Mass Ratio}

From Eqn. (10), the ratio of headspace-gas mass is

$$
\frac{N}{N_{0}}=\frac{N_{0}+\Delta N}{N_{0}}
$$

During this brief temperature excursion there were no samples withdrawn, nor were any calibrations made to the pressure transducer. The event is of sufficiently short duration that the quantity of any gases that may have been generated is fully negligible. Therefore, the headspacegas mass could only have changed if the quantity of gas dissolved in the brine changed as a result of solubility variation from the new conditions. The increased brine temperature will lower the equilibrium concentration of dissolved gas, although the accompanying increase in pressure will tend to counteract this to some degree. Equations (22) through (26) provide the basis for evaluating the equilibrium gas-solubility effect. Eqn. (26) implicitly includes the quantity (moles) of liquid, so an iterative solution with the headspace volume determination is required to accurately quantify the mass ratio. A few iterations yields the solution.

Were there no variation in the gas solubility, the term $\Delta N$ would be identically zero, so the mass ratio before and after the step temperature increase would simply be unity. In accordance with the 
discussion in Section 5.2.3, variation in the headspace-gas mass as a result of any solubility variation can be ignored. So, for this example, the headspace-gas mass ratio in this example simply has a value of unity:

$$
\frac{N}{N_{0}}=1
$$

\subsubsection{Headspace-Volume Ratio}

Table 4 shows the temperature and pressure measurements taken during each of the four headspace-gas-sample measurements for this test container, along with the measured glovebox temperatures. For these measurements the temperatures were constant throughout the sampling event. The shaded portion of the table gives some quantities derived from these measurements. The equation number, or other reference that is the source of the calculation, is shown in parentheses for each column. These include the bulk headspace-gas temperature, as calculated for each sample event from the one-dimensional thermal model, the vapor pressure of water, calculated at the bulk headspace-gas temperature, and the compressibility factors for the initial and final conditions during the gas expansion. The headspace volumes at the prevailing measurement conditions are shown in the last column (see Sections 6.2.1 and 6.2.2).

Table 4. Raw Headspace Volume Measurements for TC-153 ${ }^{\mathrm{a}}$

\begin{tabular}{|c|c|c|c|c|c|c|c|c|c|c|}
\hline No. ${ }^{b}$ & $\begin{array}{c}P_{1} \\
{[\mathrm{MPa}]} \\
\text { (psia) }\end{array}$ & $\begin{array}{c}P_{2} \\
{[\mathrm{MPa}]} \\
\text { (psia) }\end{array}$ & $\begin{array}{c}T_{\mathrm{RTD}} \\
{[\mathrm{C}]}\end{array}$ & $\begin{array}{l}T_{\mathrm{GB}} \\
{[\mathrm{C}]}\end{array}$ & $\begin{array}{c}T_{\mathrm{EX}}{ }^{\mathrm{c}} \\
{[\mathrm{C}]} \\
(60)\end{array}$ & $\begin{array}{c}T_{\mathrm{HS}}{ }^{\mathrm{d}} \\
{[\mathrm{C}]} \\
(59)\end{array}$ & $\begin{array}{c}P_{\mathrm{v}}{ }^{\mathrm{e}} \\
{[\mathrm{kPa}]} \\
\text { (psia) } \\
\text { (Ref. 1) }\end{array}$ & $\begin{array}{c}z_{1} \\
(55)\end{array}$ & $\begin{array}{c}z_{2} \\
(55)\end{array}$ & $\begin{array}{l}V_{\mathrm{HS}} \\
{[\mathrm{ml}]} \\
(46)\end{array}$ \\
\hline-02 & $\begin{array}{r}15.100 \\
(2190.1)\end{array}$ & $\begin{array}{r}15.033 \\
(2180.4)\end{array}$ & 29.80 & 22.1 & 3.27 & 28.66 & $\begin{array}{c}3.930 \\
(0.570)\end{array}$ & "1.026620 & 1.026275 & 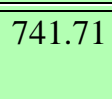 \\
\hline-03 & $\begin{array}{r}14.973 \\
(2171.7)\end{array}$ & $\begin{array}{r}14.905 \\
(2161.8)\end{array}$ & 30.55 & 27.9 & 2.08 & 30.39 & $\begin{array}{c}4.344 \\
(0.630)\end{array}$ & 1.027114 & 1.026764 & 719.57 \\
\hline-04 & $\begin{array}{r}14.927 \\
(2165.0)\end{array}$ & $\begin{array}{r}14.860 \\
(2155.3)\end{array}$ & 30.60 & 28.6 & 2.05 & 30.60 & $\begin{array}{c}4.392 \\
(0.637)\end{array}$ & 1.027017 & 1.026675 & 731.83 \\
\hline-05 & $\begin{array}{r}14.863 \\
(2155.7)\end{array}$ & $\begin{array}{r}14.795 \\
(2145.9)\end{array}$ & 30.40 & 27.7 & 2.09 & 30.23 & $\begin{array}{c}4.302 \\
(0.624)\end{array}$ & 1.026444 & 1.026101 & 720.73 \\
\hline
\end{tabular}

a. Columns shown unshaded are measurements; those shaded are calculated values, with the source (equation number or reference) shown in parentheses.

b. Measurement No. -01 was made at low pressure and therefore not included in table.

c. $T_{\mathrm{EX}}$ is an estimate of the local environment temperature within the glovebox above that measured by the glovebox RTD (see Appendix D).

d. Bulk headspace-gas temperature calculated from the one-dimensional thermal model.

e. Vapor pressure of water calculated at the temperature of the headspace gas. 
For the temperature step from $29.6^{\circ} \mathrm{C}$ to $39.8^{\circ} \mathrm{C}$ the average expansion coefficient, $\bar{\beta}$, for the brine is $0.383 \mathrm{e}-3^{\circ} \mathrm{C}^{-1}$ (Fig. B6). Table 5 gives the brine expansion coefficient (from Fig. B6) and the headspace volumes adjusted to the reference conditions, calculated as shown in Eqn. (50). These are averaged, per Eqn. (51), to yield the best-estimate value of $730.27 \mathrm{ml}$ at the reference conditions. The container volume is $7272 \mathrm{ml}$ (Section 6.1.1). Therefore, the brine volume at the reference conditions, $V_{l_{0}}$, is $7272-730.27 \mathrm{ml}$ or $6542 \mathrm{ml}$.

The linear expansion coefficient, $\mu$, for the container has a value of $11.3 e-6 \mathrm{C}^{-1}$ (Ref. 10). At the new conditions following the step temperature increase, Eqn. (41) gives the test container volume increase as

$$
\begin{aligned}
\Delta V_{C} & =V_{C_{0}}\left(e^{3 \bar{\propto} \Delta T_{R T D}}-1\right) \\
& =7272 m l\left(e^{3\left(11.3 E-6^{\circ} C^{-1}\right)\left(39.8-29.6^{\circ} C\right)}-1\right) \\
& =2.52 m l
\end{aligned}
$$

Table 5. Adjusted Headspace Volume Measurements for TC-153

\begin{tabular}{|l|c|c|c|c|c|}
\hline $\begin{array}{c}\text { Measurement } \\
\text { No. }\end{array}$ & $\begin{array}{c}V_{\mathrm{HS}-\mathrm{j}} \\
{[\mathrm{ml}]} \\
(46)\end{array}$ & $\begin{array}{c}D T_{\text {RTD-j }} \\
{[\mathrm{C}]}\end{array}$ & $\begin{array}{c}\mathrm{T}_{\text {RTD-j }} \text { ave } \\
{[\mathrm{C}]}\end{array}$ & $\begin{array}{c}\beta_{\text {ave }} \\
{\left[\mathrm{C}^{-1}\right]} \\
(\text { Fig. B6) }\end{array}$ & $\begin{array}{c}V_{\mathrm{HS}-0 \mathrm{j}} \\
{[\mathrm{ml}]} \\
(50)\end{array}$ \\
\hline \hline-02 & 741.71 & 0.20 & 29.70 & $0.374 \mathrm{E}-3$ & 742.20 \\
\hline-03 & 719.57 & 0.95 & 30.08 & $0.375 \mathrm{E}-3$ & 721.90 \\
\hline-04 & 731.83 & 1.00 & 30.10 & $0.375 \mathrm{E}-3$ & 734.28 \\
\hline-05 & 720.73 & 0.80 & 30.00 & $0.375 \mathrm{E}-3$ & 722.69 \\
\hline Sum & -- & -- & & -- & 2921.08 \\
\hline Ave. $\left(V_{\mathrm{HS}^{-}}\right)$ & -- & -- & & -- & 730.27 \\
\hline
\end{tabular}

The brine/waste volume increase, from Eqn. (43), is

$$
\begin{aligned}
\Delta V_{l} & =V_{l_{0}}\left(e^{\bar{\beta} \Delta T_{R T D}}-1\right) \\
& =6542 \mathrm{ml}\left(e^{0.383 E-3^{\circ} C^{-1}\left(39.8-29.6^{\circ} \mathrm{C}\right)}-1\right) \\
& =25.61 \mathrm{ml}
\end{aligned}
$$


So the headspace volume increase (Eqn. (38) is

$$
\Delta V_{H S}=\Delta V_{C}-\Delta V_{l}=2.52-25.6=-23.09 \mathrm{ml}
$$

The headspace volume ratio then becomes

$$
\frac{V_{H S_{0}}}{V_{H S}}=\frac{V_{H S_{0}}}{V_{H S_{0}}+\Delta V_{H S}}=\frac{730.27 \mathrm{ml}}{730.27 \mathrm{ml}-23.09 \mathrm{ml}}=1.03265
$$

\subsubsection{Predicted Pressure}

With the ratios determined in the preceding sections, the predicted total pressure following the temperature step change can be immediately computed from Eqn. (7):

$$
\begin{aligned}
P-P_{v} & =\left(P-P_{v}\right)\left(\frac{Z}{z_{0}}\right)\left(\frac{N}{N_{0}}\right)\left(\frac{V_{H S_{0}}}{V_{H S}}\right) \frac{T_{g}}{T_{g_{0}}} \\
P-6.047 \mathrm{kPa} & =(14.615 \mathrm{MPa}-3.847 \mathrm{kPa})(1.01032)(1.0)(1.03265)(1.02658) \\
& =14.611 \mathrm{MPa} \cdot 1.07104 \\
& =15.649 \mathrm{MPa} \quad(2269.7 \mathrm{psia})
\end{aligned}
$$

So the predicted total pressure, i.e., the gas pressure plus vapor pressure, is

$$
P=15.649 \mathrm{MPa}+6.047 \mathrm{kPa}=15.655 \mathrm{MPa}(2270.6 \mathrm{psia})
$$

This is quite close to the measured value of $15.663 \mathrm{MPa}$ (2271.7 psia).

The relative contribution of each of the thermodynamic terms to the pressure rise can be quickly assessed for this example by examining the pressure calculation. Each of the four thermodynamic ratios has a value of unity or exceeds unity. The excesses, summed, exceed unity by a total of 0.06955 . The headspace volume ratio is seen to be the biggest contributor to the pressure rise. This term contributes $0.03265 / 0.06955$, or about $47 \%$ of the total excess, substantially greater than the $0.02658 / 0.06955$, or $38 \%$, contribution from the headspace-gas temperature. The compressibility variation contributes the remaining $15 \%$. Recall that the volume ratio was the consequence of the thermal expansion of the brine/waste, lessened somewhat by the simultaneous thermal expansion of the test vessel, Eqn. (38). Little wonder then, that the earlier analysis of the data that considered only the direct temperature effect (even when aided by the erroneous assumption that the headspace-gas temperatures were the same as those measured by the test-container RTD) had failed so badly to predict the system response.

This computation demonstrates the validity of the GGE data. The remainder of this chapter further clarifies the calculation methodology. 


\subsubsection{Compensated Pressure}

The treatment shown in the preceding section is a predictive form of the calculation: the original pressure and the variations of temperature and headspace-gas mass were used to predict the new pressure following some change. An alternate, but fully equivalent, point of view is to start with the actual final pressure measured and then purge that value of the effects of the temperature and/ or headspace-gas-mass variations. With this approach, these effects will have been factored out, yielding an estimate of the original pressure. This approach is referred to in this report as the compensated-pressure form of the methodology. The compensated value provides an estimate of the reference pressure when there is no gas generation. As before, terms with zero subscript refer to the reference conditions, others refer to the new conditions following the temperature step. From Eqn. (8) and the values obtained in Section 9.1.5, the compensated pressure for the example becomes

$$
\begin{aligned}
P_{\text {comp }}-P_{v_{0}} & =\left(P-P_{v}\right)\left(\frac{Z_{0}}{Z}\right)\left(\frac{N_{0}}{N}\right)\left(\frac{V_{H S}}{V_{H S_{0}}}\right)\left(\frac{T_{g_{0}}}{T_{g}}\right) \\
P_{\text {comp }}-3.847 k \dot{P} a= & (15.663 M P a-6.047 \mathrm{kPa}) \frac{1}{1.01032} \frac{1}{1.0} \frac{1}{1.03265} \frac{1}{1.02658} \\
& =(15.657 \mathrm{MPa} a) \frac{1}{1.07104} \\
& =14.618 \mathrm{MPa}(2120.2 \mathrm{psia})
\end{aligned}
$$

So the compensated total pressure is found to be

$$
P_{\text {comp }}=14.622 \mathrm{MPa}(2120.7 \text { psia })
$$

As expected, this compares favorably to the actual pre-step (in this case the reference value) pressure of 14.615 MPa (2119.7 psia).

The above form of the example demonstrates the capability of the analytical methodology to reasonably accurately recover the original pressure of $14.615 \mathrm{MPa}$ (2119.7 psia) after compensating the measured pressure for, i.e., nullifying, the effects of temperature variation. Comparing the $P_{\text {comp }}$ expression above to the adjusted pressure $P_{\text {adj }}$ defined in Eqn. (28), it is seen that $P_{\text {adj }}$ and $P_{\text {comp }}$ are exactly the same.

\subsubsection{Mechanical Work}

The role of mechanical-energy conversion on the headspace-gas temperature was discussed in Section 8.2, and can be demonstrated by this example. The headspace volume change was calculated in Section 9.1.4 to be $-23.09 \mathrm{ml}$. With the cross-sectional area of the test container, $181.1 \mathrm{~cm}^{2}$, the displacement of the brine surface resulting from the brine expansion would have been only $1.3 \mathrm{~mm}$. However, a force of approximately 274,680 N (61,750 lbf) must be exerted to displace the brine/waste surface against the high gas pressure that prevails. 
For illustration purposes, the integral in Eqn. (65) is simply approximated by using the average pressure, and the headspace-gas mass is written using the state equation. Then the temperature rise, $D T_{\mathrm{ME}}$, that results from the conversion of mechanical energy (alone) to internal energy for an adiabatic process is

$$
\Delta T_{M E}=-\frac{1}{M_{g} c_{v}} \int_{1}^{2} P d V \approx-\frac{z_{0} R_{U} T_{g_{0}}}{P_{g_{0}} V_{H S_{0}} c_{v}[M W]} P_{a v e} \Delta V_{H S}
$$

The values for each of these terms from the previous example are substituted and the thermodynamic properties appropriate to nitrogen at room temperature are used:

$$
\begin{aligned}
z_{0} & =1.0238 \\
T_{g_{0}} & =28.29+273.15=301.44 \mathrm{~K} \\
\Delta V_{H S} & =-23.09 \mathrm{ml} \\
V_{H S} & =730.07 \mathrm{ml} \\
P_{\text {ave }} & =0.5(14.615+15.663) \mathrm{MPa}=15.139 \mathrm{MPa} \quad \text { (2195.7psia }) \\
R_{U} & =0.08206 \mathrm{~atm}-\text { litre } / \mathrm{g}-\mathrm{mole} / \mathrm{K} \\
C_{v} & =7.08 \mathrm{E}-3 \mathrm{~atm}-\text { litre } / \mathrm{g} / \mathrm{K} \\
{[M W] } & =28 \mathrm{~g} /(\mathrm{g} \text {-mole })
\end{aligned}
$$

Therefore, the mechanical work performed in compressing the headspace gas is found to raise the gas temperature approximately $4.13^{\circ} \mathrm{C}$. This calculation over-estimates $D T_{\mathrm{ME}}$ somewhat, because the average pressure in Eqn. (66) was calculated using the final pressure, not just that portion of the pressure increase that resulted from the mechanical compression. The actual value can be found from the thermodynamic relations for the isentropic compression implied in this example. For such a process in an ideal gas, $P V^{\mathrm{g}}$ is a constant. For the same $D V_{\mathrm{HS}}$, this shows that the mechanical compression, alone, causes the pressure to increase to $15.301 \mathrm{MPa}$ (2219.2 psia). The temperature rise from the mechanical work is found to be about $4.08^{\circ} \mathrm{C}$.

This is the temperature rise that would occur if the $23.09 \mathrm{ml}$ compression of the headspace gas had occurred adiabatically. Heat transfer from the brine/waste to the headspace gas and then out to the glovebox environment provides the further temperature increase to the final equilibrium temperature of $36.30^{\circ} \mathrm{C}$, i.e., a total temperature rise of $36.30-28.29^{\circ} \mathrm{C}=8.01^{\circ} \mathrm{C}$.

\subsection{Adjustment of the Pressures in the Blank, Experiment-Control Test Containers}

The adjusted pressure was computed for each data point for the two non-waste-bearing test containers, No. 152 and 153. The calculations required and made at each data point are the same as that shown in the example in Section 9.1. These are relatively simple calculations, but the plethora of data points and the several iteration routines that are required dictated the use of the 
computer program. The final evolution of the special program developed, GGE7b1, is described in Appendix D. Temperature and pressure measurements were taken hourly, yielding approximately 26,000 data points for each test container over the more than three years of data considered in this report. Each of these data points were processed to provide the results presented here.

The computation was begun by appending to each point in the data file an estimate of the local glovebox temperature per the algorithm described in Eqn. (60). The FORTRAN computer program reads this data file, and, using the methods described in Sections 5.0 through 8.0 of this report, determines the thermodynamic-parameter ratios needed to evaluate the adjusted pressure from Eqn. (28). The adjusted pressures are written to an unformatted-text output file, which, for convenience, is subsequently inserted into a Microsoft EXCEL program. The change in mole fraction of the generated gases relative to the reference point, $d X_{\text {gen }}$, is calculated from Eqn. (33) and added to the spreadsheet. Finally, the adjusted pressures and mole fractions are plotted using the spreadsheet plotting utility.

Figure 18 and Fig. 19 show the temperatures for the two blank test containers, TC-152 and TC-153. The points at which headspace-gas samples were taken are marked on the figures, as are the points at which RTD calibration checks were made. Figure 20 and Fig. 21 show the corresponding pressure data. Superimposed on the measured pressure plots are the adjusted pressures as computed by the GGE7b1 FORTRAN program. These are relative to the arbitrarily selected reference point identified in the title line on each plot. The points at which headspacegas samples were extracted and the pressure-instrument calibrations made are shown on the pressure plots. The drop in pressure of approximately 55-70 kPa (8-10 psi) that occurred each time a headspace gas sample was drawn is evident in the pressure data record. 


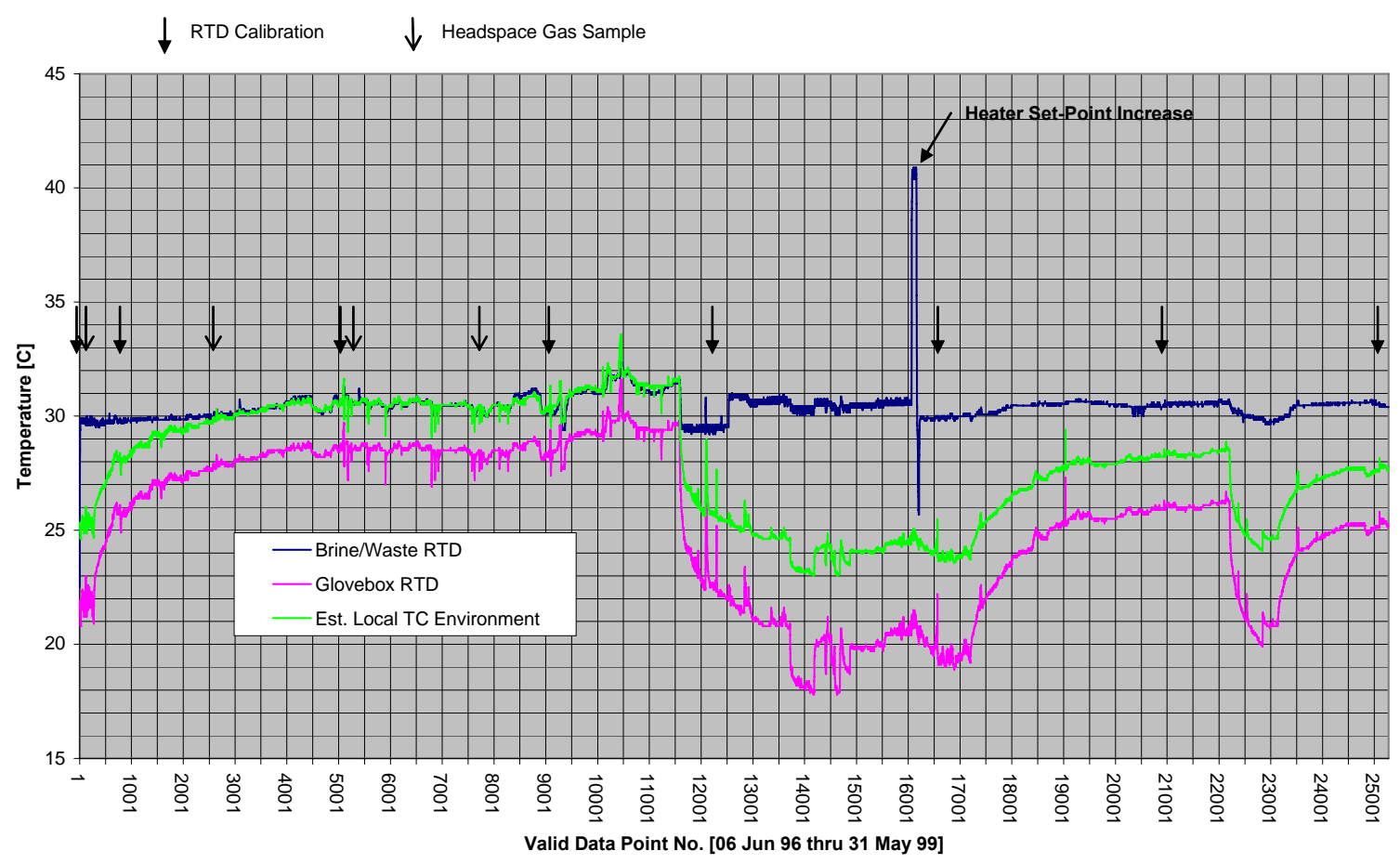

Fig. 18. Temperatures, TC-152

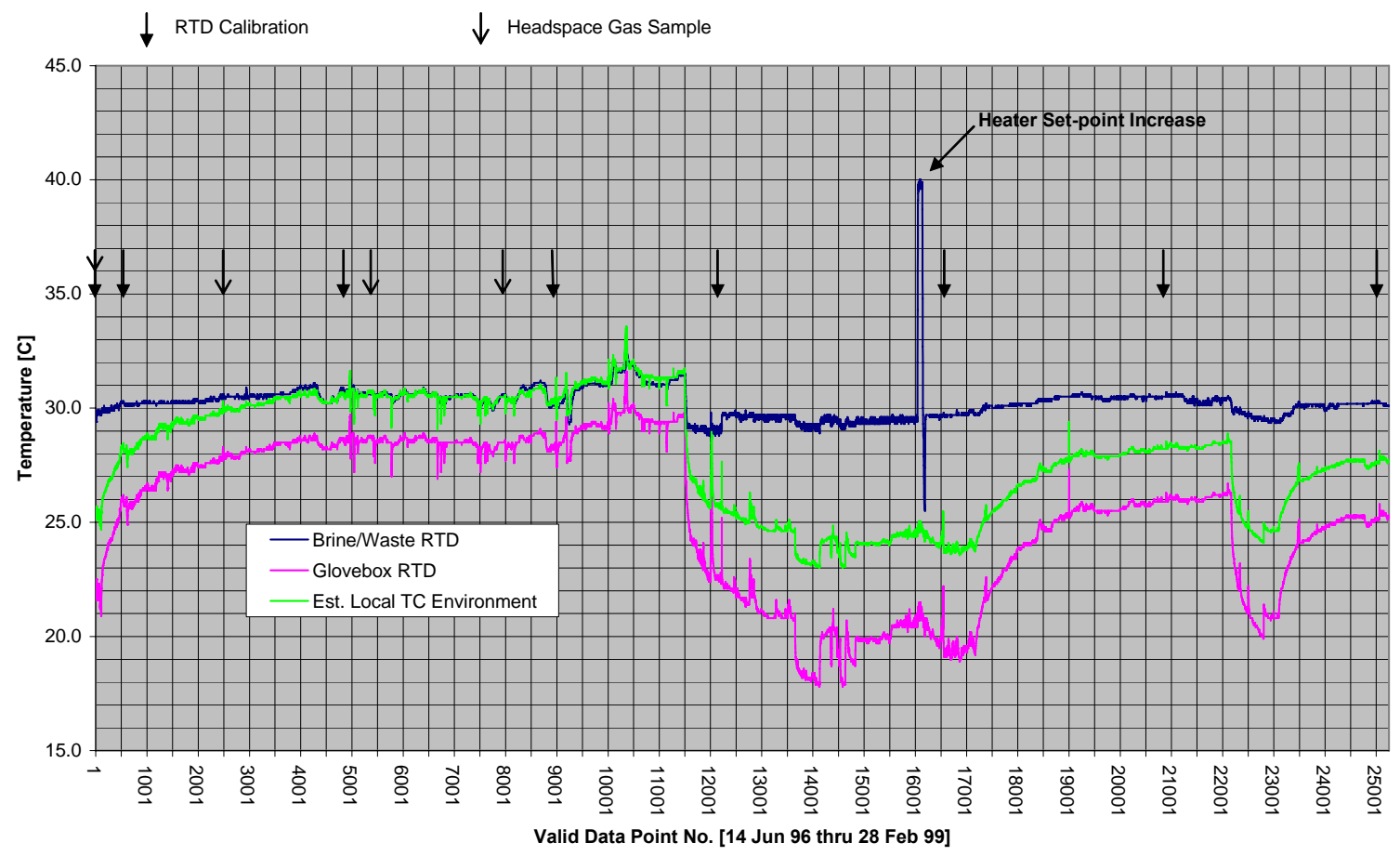

Fig. 19. Temperatures, TC-153 


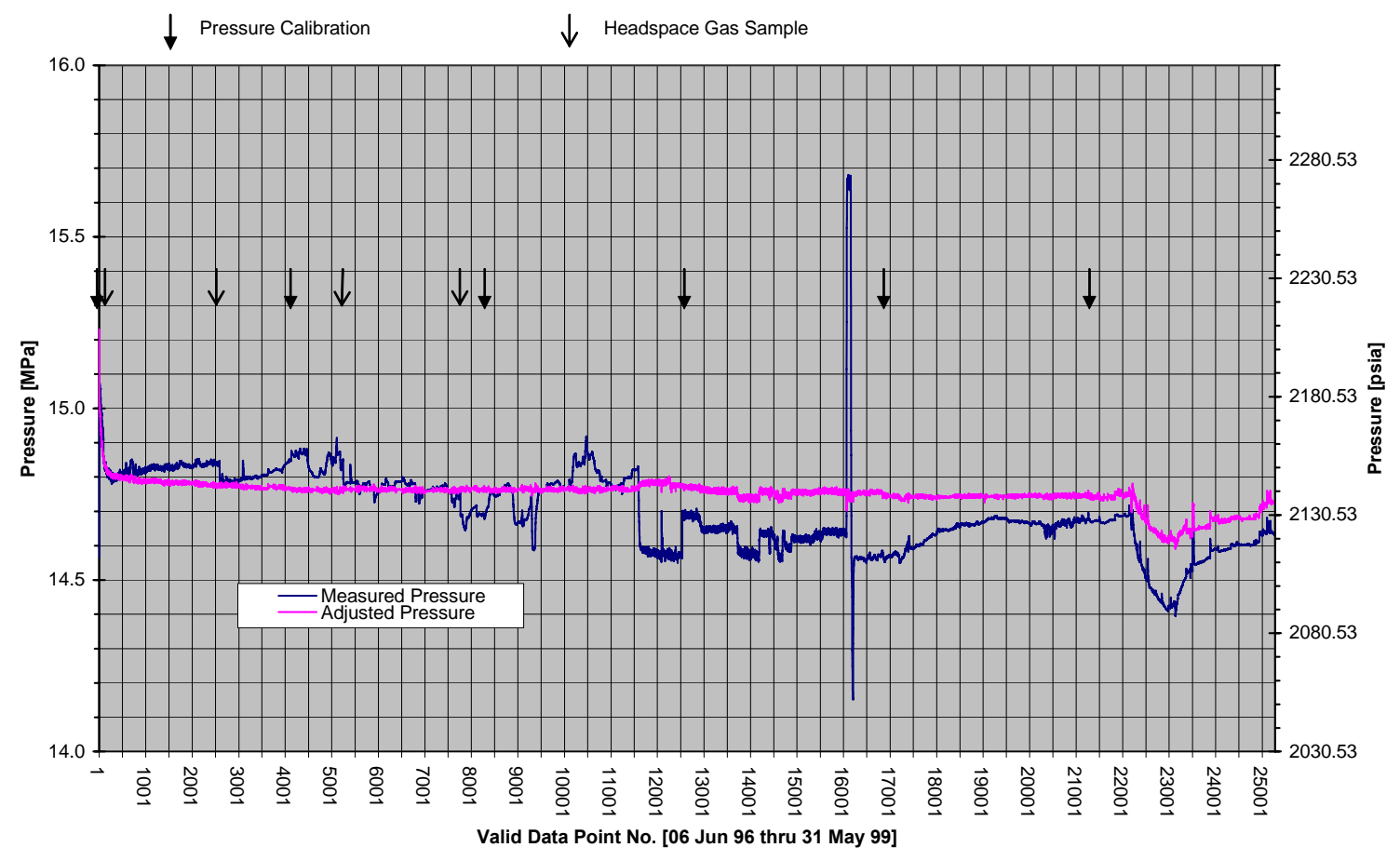

Fig. 20. Pressures, TC-152 (Ref. Pt. 10001)

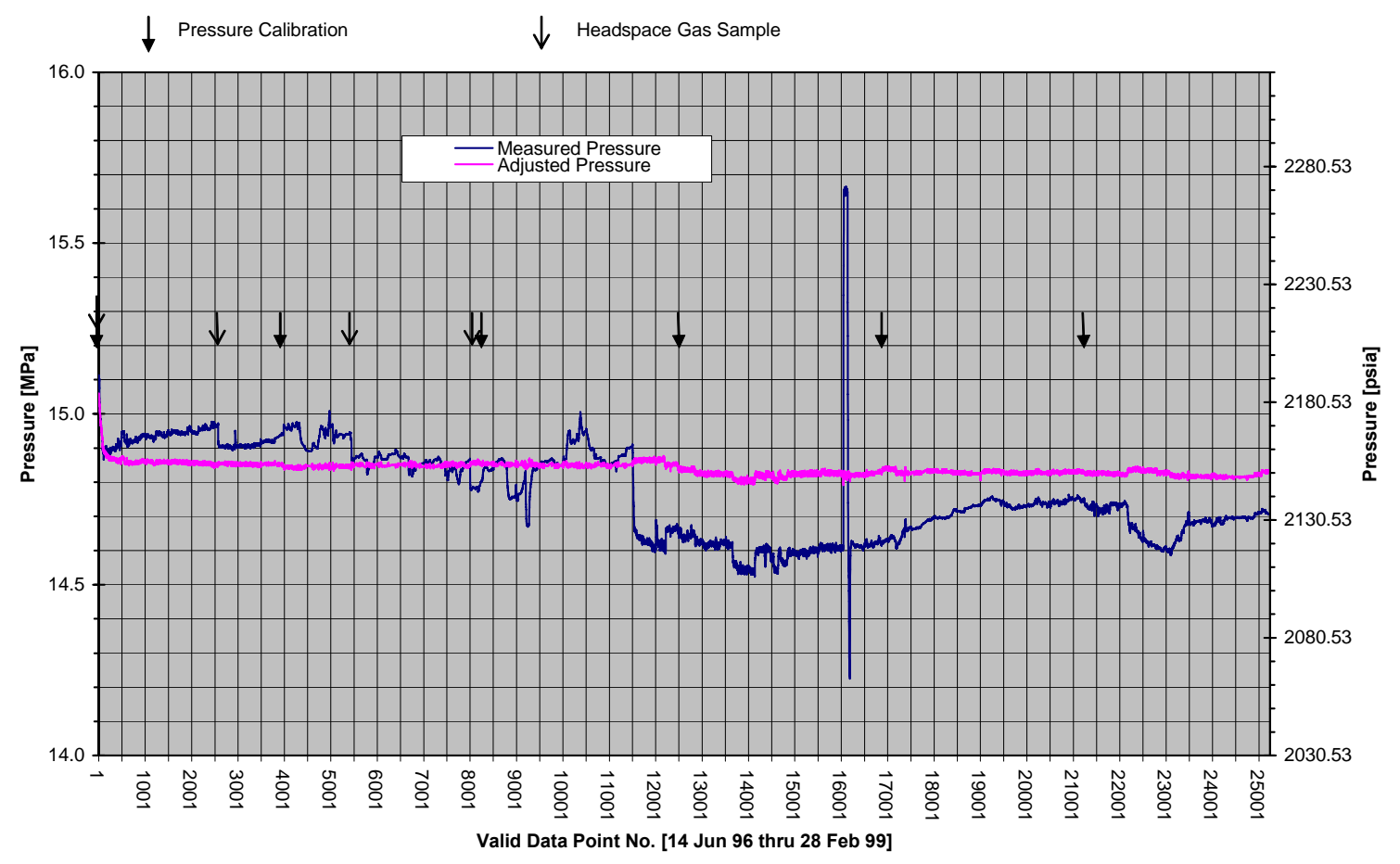

Fig. 21. Pressures, TC-153 (Ref. Pt. 10001) 
The computed adjusted-pressures for both blank test containers show an approximately constant value, as anticipated, and the pressure rise that occurred at the temperature spike addressed earlier in this section is duplicated by the program. The variation from a constant value is modest, and this variation is indicative of the uncertainty that must be accepted when using the analysis methodology on field data. Considering the plethora of uncertainties and assumptions imbedded in the data-recovery methodology, the results are satisfactory. Imperfections inherent in the methodology are discussed in Section 13.0. Notwithstanding these inherent imperfections, the adjusted pressures for these two test containers are the actual pressures that would have prevailed had there been no temperature variation in the headspace gas nor any changes to the headspacegas masses from sampling or PXD calibrations. The adjusted pressure plots reveal the pressure variation that remains after analytical compensation for the headspace temperature and mass changes.

The mole fractions of generated gases over the experiment are depicted in Fig. 22 and Fig. 23. The mole fraction traces also, of course, reflect the imperfections in the analysis and any discrepancies that are imbedded in the data, but it is quite clear that there are no perceptible indications of long-term gas-generation trends.

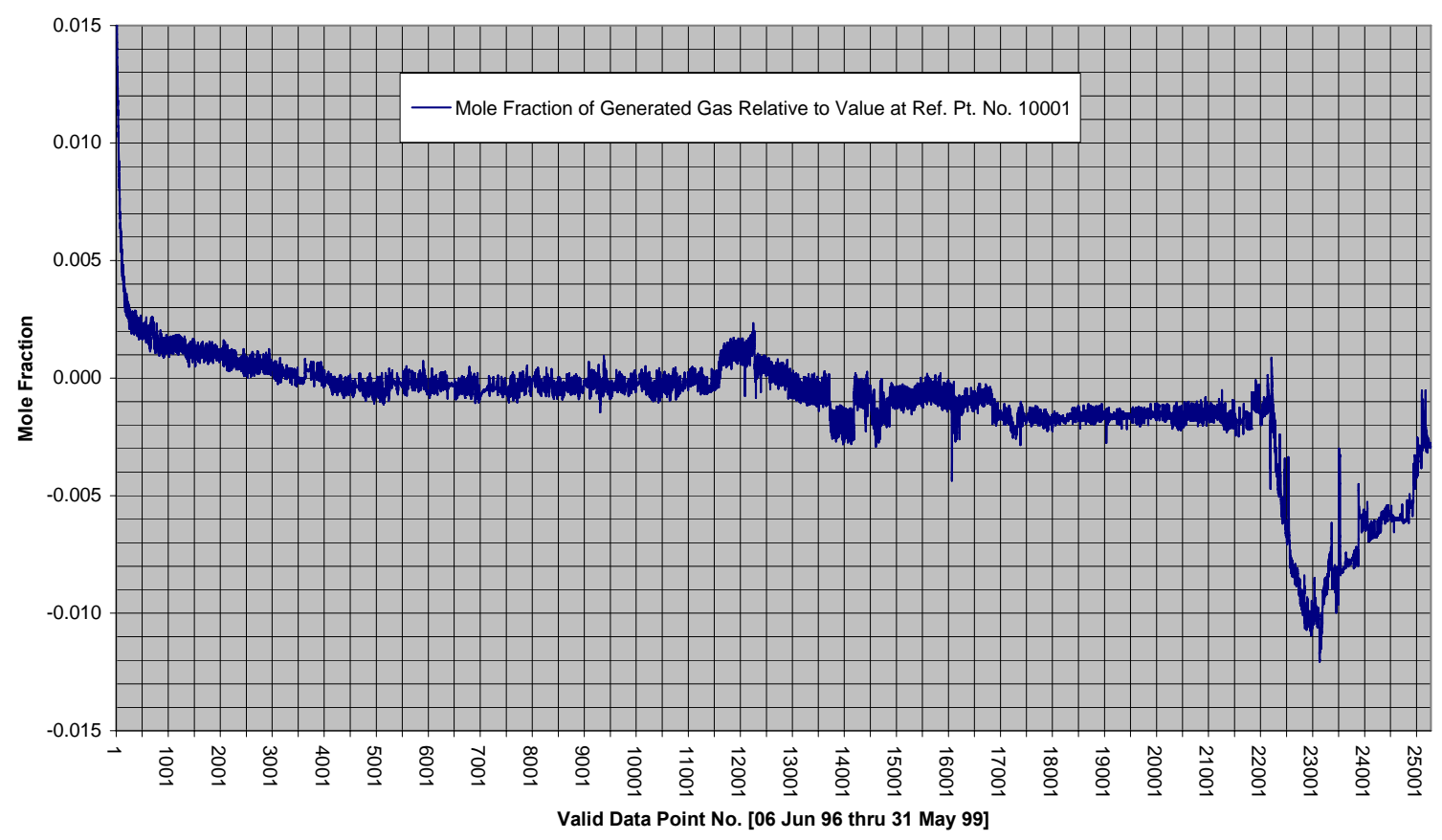

Fig. 22. Gas Generation in TC-152 


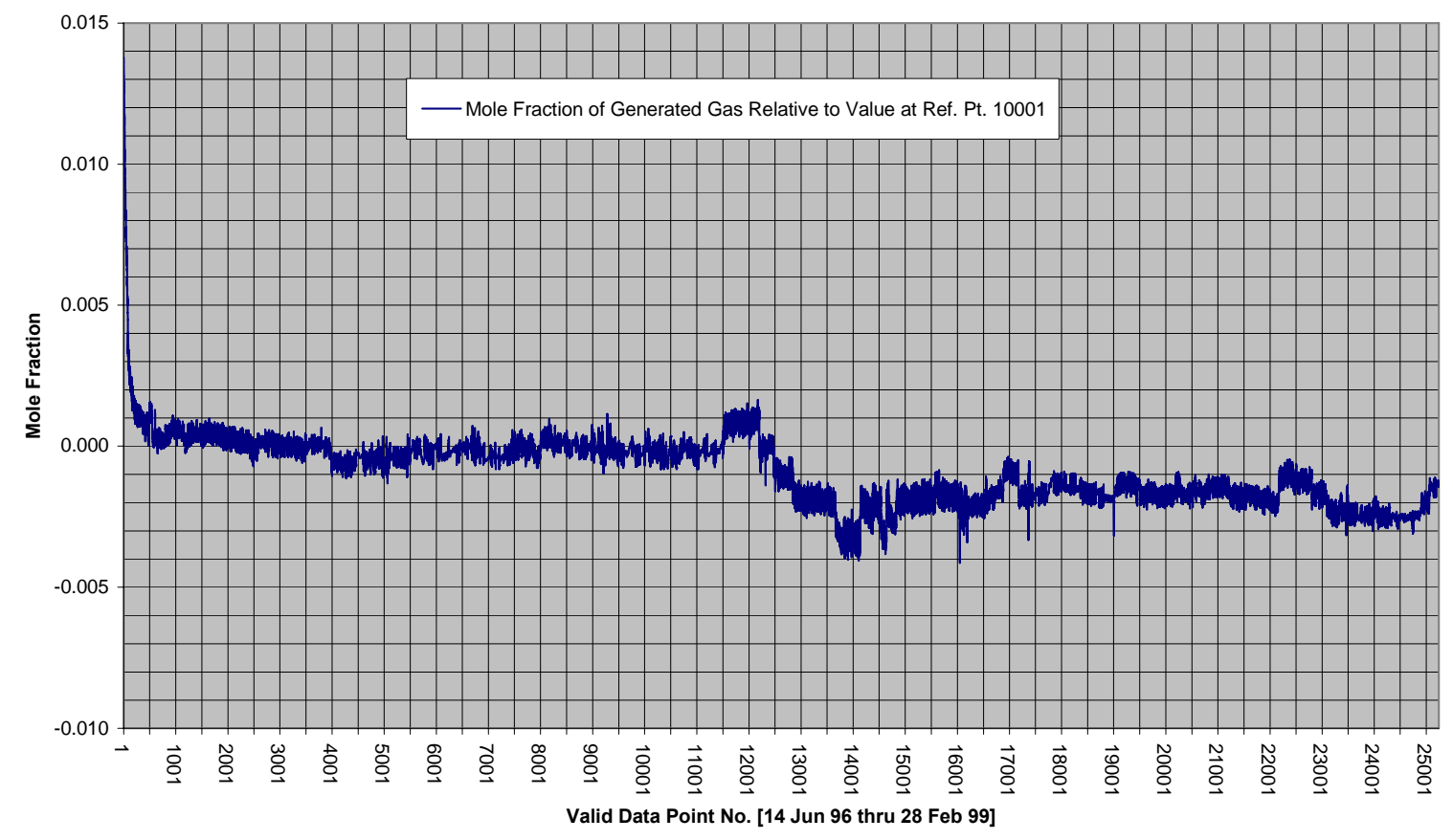

Fig. 23. Gas Generation, TC-153

\section{Recovery of the Archived Pressure Measurements}

The previous section established both the validity of the experimental data as well as the usefulness of the analyses. Attention is now turned to recovering the extensive data that was collected during the first 3.25 years of the experiment. This requires a slight modification to the methodology. The key new features are described in this section.

\subsection{Determination of the Brine/Waste Thermal Expansion Coefficient}

The calculations in Section 9.0 were made using a value for the brine expansion coefficient that was determined from dedicated measurements made on the brine in a separate experiment (Appendix B). Analogous measurements were not made, and are probably not possible to make with any reasonable accuracy, for the various brine/waste combinations. To do so would require duplicating the brine/waste in the off-line experiments. Not only would this be costly and time consuming, there is little likelihood that the physical arrangement of these mixtures could be duplicated to provide meaningful data. For example, bubbles trapped within the waste matrix play a profound role in the overall "elasticity" of the material, and duplication of the bubble quantity and distribution that may be in the actual test containers would be impossible.

A different approach was needed, and the one devised was, in fact, much simpler. A step temperature change was deliberately introduced into each test container's heater controller. This was limited to one or two test containers at a time, so as to not significantly affect the overall glovebox heat load. The system was not further disturbed for the 3-4 days required to ensure the establishment of a new equilibrium operating point. The test-container temperature and corresponding pressure response were then duly measured and recorded. This provided data 
similar to the incidental temperature spike that was addressed in the data-validation example in Section 9.1. Based on the experience found in the validation examples, an accurate value for the brine/waste expansion coefficient, $\beta$, would be expected to yield an accurate prediction of the adjusted pressure response. Now, instead of applying the analyses to predict the pressure response, the measured response, itself, was used to back the brine/waste expansion coefficient out of the analyses. That is, the predicted pressure for the induced temperature spike was forced to match the measured response, allowing the value of $\beta$ to be determined from the analyses. Once this parameter was obtained from the measured pressure response to the induced temperature step, it could then be used with the entire set of archived data for that test container to calculate the adjusted pressures at all other data points.

The analytical development for this approach is as follows. Let subscript " 1 " refer to the conditions just prior to the induced temperature step, and subscript " 2 " refer to the new equilibrium conditions following the step. Equations (36) through (38) relate the headspace volume following the step to that just before the temperature step as

$$
V_{H S_{2}}=V_{H S_{1}}+\delta V_{C_{\text {step }}}-\delta V_{l_{\text {step }}}
$$

Similar to Eqn. (41) and (43), expressions for $d V_{\mathrm{C} \text {-step }}$ and $d V_{\text {l-step}}$, respectively, can be derived for the conditions of the step change in the headspace volume.

From Eqn. (41),

$$
\delta V_{C_{\text {step }}}=V_{C_{1}}\left(e^{3 \bar{\alpha}_{\text {step }} \delta T_{\text {step }}}-1\right)
$$

The subscript "step" emphasizes that the associated terms refer specifically to the changes that occur between the pre- and post-induced-step conditions, i.e., over the transition from state 1 to state 2. For example, this report generally uses $\mathrm{D} T_{\mathrm{RTD}}$ to refer to the difference between the container RTD temperature (i.e., brine/waste temperature) at the current point minus the RTD temperature at the reference point. When the lower-case delta symbol (d) is used it refers simply to some change. For the specific case where the variable is subscripted step, it refers to the step conditions (the value after the step minus the value just prior to the step. Were the reference point always taken at the pre-step conditions, there would obviously be no difference in the nomenclature; however, it is preferred to keep this development general, allowing the reference point for the overall calculation to be arbitrarily selected independently of the induced step measurements.

The container volume at the pre-step conditions can be related back to the corresponding volume at the overall reference point by using Equations (39) and (41) to obtain

$$
V_{C_{1}}=V_{C_{0}} e^{3 \bar{\alpha}_{1-0} \Delta T_{1-0}}
$$


The subscript here emphasizes that the terms refer to the interval or difference from the pre-step conditions to the overall reference point. This relation can be substituted into Eqn. (68) to find the change in container volume when referenced to the volume at the overall reference point.

Similarly for the brine/waste, from Eqn. (43), $d V_{l \text {-step }}$ becomes

$$
\begin{aligned}
\delta V_{l_{\text {step }}} & =V_{l_{1}}\left(e^{\bar{\beta}_{\text {step }} \delta T_{\text {step }}}-1\right) \\
& =\left(V_{C_{1}}-V_{H S_{1}}\right)\left(e^{\bar{\beta}_{\text {step }} \delta T_{\text {step }}}-1\right)
\end{aligned}
$$

The headspace volumes at the conditions both before and following the step can be written using the equation of state:

$$
V_{H S}=\frac{z N R_{U} T_{g}}{P_{g}}
$$

Upon combining Equations (67) through (71) and rearranging, the average value of the expansion coefficient over the step, $\beta_{\text {step }}$, is found to be

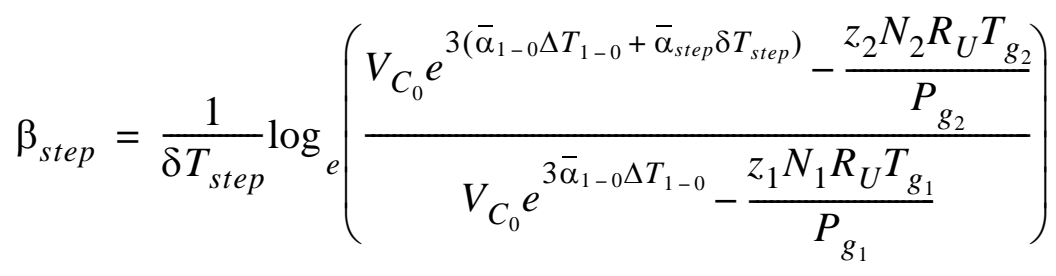

There is a temperature dependency for the $§$ term. This is evident from Fig. B6 in Appendix B for tests conducted on pure brine, as well as for pure water shown in Fig. B5; hence, the inclusion of the average value in Eqn. (70) (note that Eqn. (68) has also been developed for the general case of a temperature-dependent $a$, although a constant value of $11.3 \mathrm{e}-6 \mathrm{C}^{-1}$ was used in all of the calculations in this report). For this reason, two separate step temperature changes were actually induced in each test container: a "high-temperature" step, obtained by increasing the testcontainer-heater set point well above the normal operating temperature; and a "low-temperature" step, obtained by reducing the heater set point to below the normal-operating level. The maximum permissible temperature was $40^{\circ} \mathrm{C}$ to avoid harming the inoculated microbes (15). An equation like that above was written for both the high-temperature and low-temperature steps. In the high-temperature-step equation, the average value of $\beta$ over the temperature step is denoted as $\beta_{\mathrm{H}}$, while for the low-temperature step the average value is denoted $\beta_{\mathrm{L}}$. Solutions to these equations yield two fixed values for $\beta$ that cover the temperature range of interest. Having determined these two values, the actual temperature-dependent value for $\beta$ at any temperature, $\mathrm{T}_{\mathrm{RTD}}$, inbetween was expressed as a linear function of $\beta_{\mathrm{H}}$ and $\beta_{\mathrm{L}}$, 


$$
\beta=\beta_{L}+\left(\beta_{H}-\beta_{L}\right) \frac{T_{R T D}-T_{R T D_{L}}}{T_{R T D_{H}}-T_{R T D_{L}}}
$$

Equation (72) cannot be solved explicitly, however, because the derived headspace-gas masses in the equation, $N_{1}$ and $N_{2}$, are, themselves, functions of $\beta$. This is shown in the following development.

The mass at state 2 is related to the mass at state 1 by

$$
N_{2}=N_{1}+\delta N_{\text {step }}
$$

where, as discussed in Section 5.2,

$$
\delta N_{\text {step }}=\delta N_{\text {sampling }}+\delta N_{p x d}+\delta N_{\text {sol }}+\delta N_{\text {gen }}
$$

There are no gas samples or pressure-transducer calibration events that occur between the current point and the reference point. And since the time frame is so short,

$$
\delta N_{\text {step }}=\delta N_{\text {solubility }}+\delta N_{\text {generation }} \approx 0
$$

The headspace-gas mass remains approximately constant during the step event, and the value can be related back to that at the overall reference point by using

$$
N_{2}=N_{1}=N_{0}+\Delta N_{1-0}
$$

As before, in Eqns. (18) and (21), the change in mass relative to that at the reference point can be written as

$$
\Delta N_{1-0}=\mp\left(\sum_{i=1}^{J} \delta N_{D_{i}}+\sum_{i=1}^{K} \delta N_{C_{i}}\right)
$$

Calculations of the various $d N$ terms were discussed in Section 5.0. The headspace-gas mass at the reference point must be computed from the equation of state,

$$
N_{0}=\frac{P_{g_{o}} V_{H S_{o}}}{z_{0} R_{U} T_{g_{0}}}
$$

Alas, it is recalled from Section 6.2.2 that the calculated headspace volume at the reference point, i.e., $\quad V_{\mathrm{HS}-0}$, is the average of the adjusted measurements made during sampling. These adjustments, per Eqn. (50), clearly depend on the value of $\beta$.

Given the transcendental character of Eqn. (72), the solution was obtained by iteration, using successive substitution. A mean headspace volume at the reference conditions, $V_{\mathrm{HS}-0}$, was first 
guessed and used in Eqn. (79). This allowed the headspace-gas mass at the step conditions to be found from Equations (77) and (78). Then the values for $\beta_{\mathrm{H}}$ and $\beta_{\mathrm{L}}$ were computed from the step relation, Eqn. (72). With these two values and Eqn. (73), the appropriate values of $\beta$ for each of the sample measurements could be computed allowing the raw headspace-volume measurements to be adjusted to the reference conditions per Eqn. (50). Then a new average headspace volume at the reference conditions was computed, Eqn. (51), and substituted back into Eqn. (79) to improve the headspace-gas mass value. The process was repeated until the reference headspace volume converged per some pre-selected convergence criterion.

Even with an extremely poor starting guess for the reference headspace volume, the solution was found to converge rapidly. For example, a relative error of less than one part in 10,000 could be obtained in just 4 or 5 iterations. The iteration process was built into the overall analysis program and was performed automatically. Once $\beta_{\mathrm{H}}$ and $\beta_{\mathrm{L}}$ were determined, the value of $\beta$ at the required temperature could be computed from Eqn. (73) for use in the analysis of each data point.

This process was performed for each test container, yielding a temperature-dependent equation for $\beta$ that was unique to each container, i.e., unique to the brine/waste mixture within each test container. The expansion coefficients so determined are listed in Table 6.

Table 6. Volumetric Coefficients of Thermal Expansion in the Brine/Waste

\begin{tabular}{|l|c|c|c|c|}
\hline $\begin{array}{c}\text { Test Container } \\
\text { No. }\end{array}$ & $\begin{array}{c}\mathrm{T}_{\text {RTD-L }} \\
{[\mathrm{C}]}\end{array}$ & $\begin{array}{c}\text { Beta-L } \\
{\left[\mathrm{C}^{-1}\right]}\end{array}$ & $\begin{array}{c}\mathrm{T}_{\text {RTD-H }} \\
{[\mathrm{C}]}\end{array}$ & $\begin{array}{c}\text { Beta-H } \\
{\left[\mathrm{C}^{-1}\right]}\end{array}$ \\
\hline \hline TC-151 & 27.3 & $1.98 \mathrm{E}-4$ & 33.8 & $2.35 \mathrm{E}-4$ \\
\hline TC-152 & 28.1 & $3.71 \mathrm{E}-4$ & 35.6 & $3.74 \mathrm{E}-4$ \\
\hline TC-153 & 27.7 & $3.62 \mathrm{E}-4$ & 34.7 & $3.67 \mathrm{E}-4$ \\
\hline TC-160 & 27.1 & $3.68 \mathrm{E}-4$ & 33.6 & $3.51 \mathrm{E}-4$ \\
\hline TC-161 & 27.2 & $3.69 \mathrm{E}-4$ & 32.9 & $3.75 \mathrm{E}-4$ \\
\hline TC-161e $^{\text {a }}$ & 27.2 & $3.70 \mathrm{E}-4$ & 33.0 & $3.77 \mathrm{E}-4$ \\
\hline TC-162 & 28.2 & $3.88 \mathrm{E}-4$ & 33.8 & $3.88 \mathrm{E}-4$ \\
\hline TC-163 & 27.9 & $4.03 \mathrm{E}-4$ & 33.9 & $3.83 \mathrm{E}-4$ \\
\hline TC-164 & 27.3 & $3.94 \mathrm{E}-4$ & 33.9 & $3.82 \mathrm{E}-4$ \\
\hline TC-165 & 27.7 & $3.64 \mathrm{E}-4$ & 32.6 & $3.83 \mathrm{E}-4$ \\
\hline TC-166 & 27.2 & $3.43 \mathrm{E}-4$ & 32.9 & $3.39 \mathrm{E}-4$ \\
\hline TC-167 & 28.6 & $3.46 \mathrm{E}-4$ & 33.1 & $3.58 \mathrm{E}-4$ \\
\hline TC-168 & 27.5 & $3.57 \mathrm{E}-4$ & 33.3 & $3.75 \mathrm{E}-4$ \\
\hline TC-169 & 27.8 & $3.67 \mathrm{E}-4$ & 33.9 & $3.81 \mathrm{E}-4$ \\
\hline TC-170 & 30.6 & $3.28 \mathrm{E}-4$ & 32.8 & $3.57 \mathrm{E}-4$ \\
\hline
\end{tabular}

a. TC-161e uses empirical calibration constants for TC-161, per discussion in Section 11.2.1. 


\subsection{Instrument Calibrations}

Regular calibration checks were made to the instruments and recorded as part of the GGE program. When calibration errors in excess of a specified threshold were detected, the instrument was adjusted. The analyses reported here accounted for all calibration corrections that had been made by assuming these to have been the result of a steady, uniform deterioration of the accuracy since the previous calibration check.

Consider an instrument that was perfectly calibrated when checked at time $t_{1}$, and some time later, say at time $t_{2}$, a new calibration check found that the instrument was out of calibration by an amount $d_{c a l}$ (whether the adjustment was actually made to the instrument channel, or not). Each data point collected with this instrument between $t_{1}$ and $t_{2}$ was corrected in the analysis methodology on the basis of an assumed uniform degradation over the interval. For example, the data in this time interval at arbitrary time $t$ was linearly corrected by an amount

$$
\delta_{\text {correction }}=\delta_{\text {cal }} \frac{t-t_{1}}{t_{2}-t_{1}}
$$

Instrument adjustments were made when the calibration error exceeded a pre-selected threshold. The calibration check was then repeated. If the calibration check showed the instrument to be operating within the allowable bounds (either the initial calibration check or that made following any adjustment) no further adjustment was made. Any residual error, however, was noted in the operation logs. These residuals then were applied to data acquired during the next interval prior to the next calibration check.

The corrections were algebraically added to the data point prior to processing the point with the analysis program. This type of data-correction was implemented for both temperature and pressure data. The calibration corrections were included in all of the adjusted pressure calculations shown in this report, including those in Section 9.2, although in only one case, discussed in Section 11.2.1, was it ever a significant factor in any of the results.

\section{Recovered Data}

Recovery of the GGE data for a given test container consisted of computing the adjusted pressure, Eqn. (28), from measurements of the pressure, brine/waste RTD temperature, and glovebox temperature that were recorded at each data point. The adjusted pressure was then used to compute the change in the mole fraction of generated gases, via Eqn. (32). These results are presented pictorially and discussed in this section.

\subsection{Analysis Results}

Figure 24-Fig. 35 show the temperatures, adjusted pressures, and mole fractions of generated gases for each of the twelve waste-bearing test containers in the GGE program. Three plots are shown for each test container. Figure numbers with a $A$ suffix are plots of temperatures. The corresponding plot for pressure carries the same figure number, but with a $B$ suffix; and the mole 
fraction of generated gases for the same test vessel has a $C$ suffix. Figures 24-Fig. 35 correspond to Test Container No. 151 and 160 through 170, respectively. Similar results for Test Containers No. 152 and 153 were presented in Fig. 18-Fig. 23 and discussed in Section 9.2. Every valid data point has been treated in each of these plots. No filtering has been applied to either the data or to the adjusted pressures.

The temperature plots, Fig. 18 and Fig. 19, and Fig. 24 through Fig. 35, suffix A, show the measurements made by resistance temperature detectors in the test container and in the glovebox. A third, intermediate, temperature trace labeled "Estimated Local TC Environment" is the estimate of the glovebox temperature in the immediate vicinity of the test container, as was discussed in Section 8.1. These plots clearly show when the test container temperatures are being dictated by the local glovebox temperature. Headspace-gas sampling points and instrument calibrations are marked on these temperature plots. It is also clear that the algorithm, Eqn. (60), used to estimate the local test-container environment is better suited to some of the test containers than to others. For example, the estimated local environment for TC-152 (Fig. 18) tracks the brine/waste temperature very well, whereas the estimated local environment for TC-161 (Fig. 26A) doesn't fit quite as well. The fit of the algorithm to the other test containers lay inbetween these two. This probably results from the influence of other nearby equipment and/ or flow patterns both within and outside of the glovebox.

The adjusted pressures, calculated per the analysis developed in this report, are superimposed on the pressure-data (suffix $B$ ) plots. Calculations for the 3.25 year period from the beginning of the tests through the end of May 1999 are provided. These figures also show the points at which the pressure instrumentation was calibrated and when headspace-gas samples were taken.

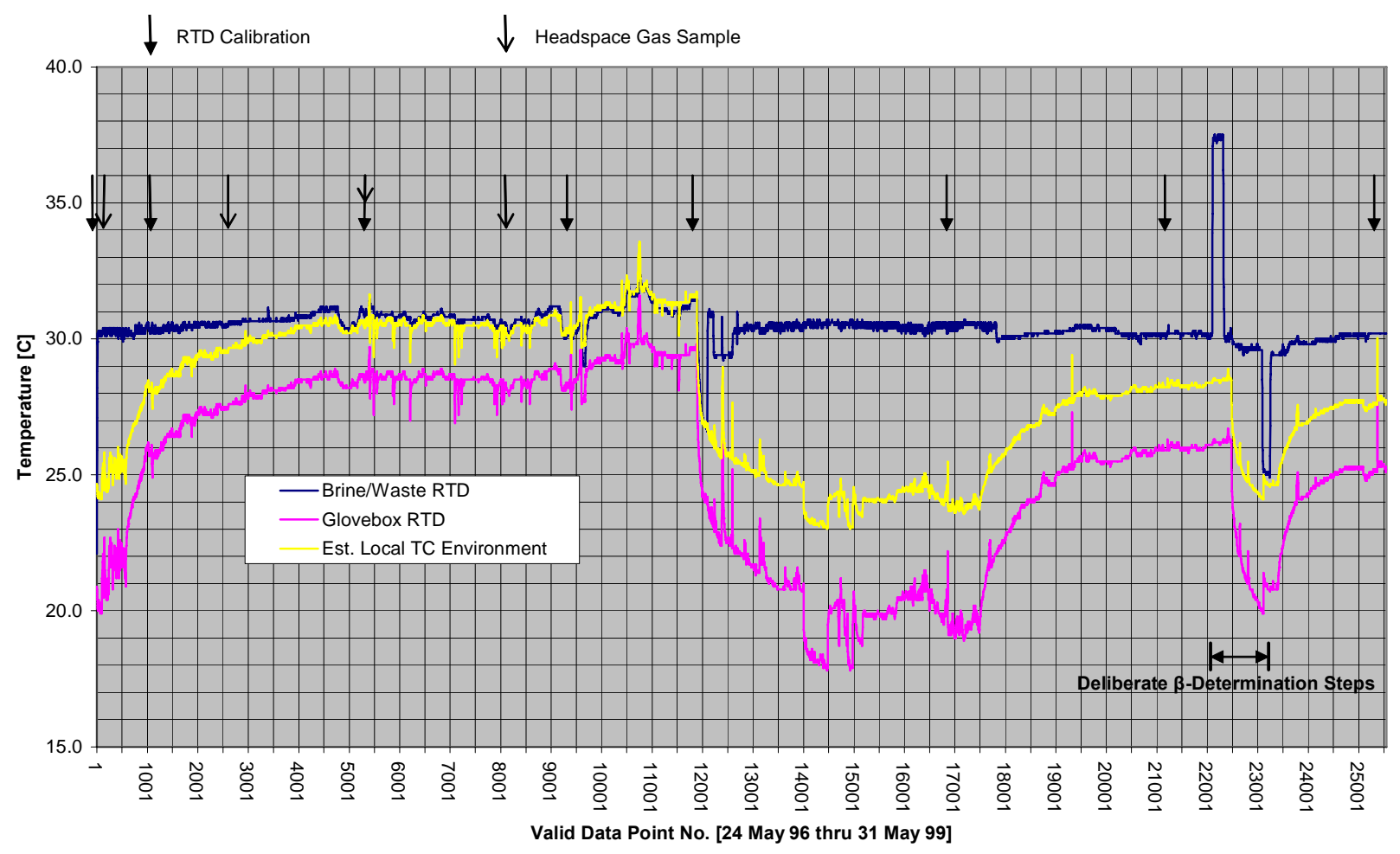

Fig. 24A. Temperatures, TC-151 


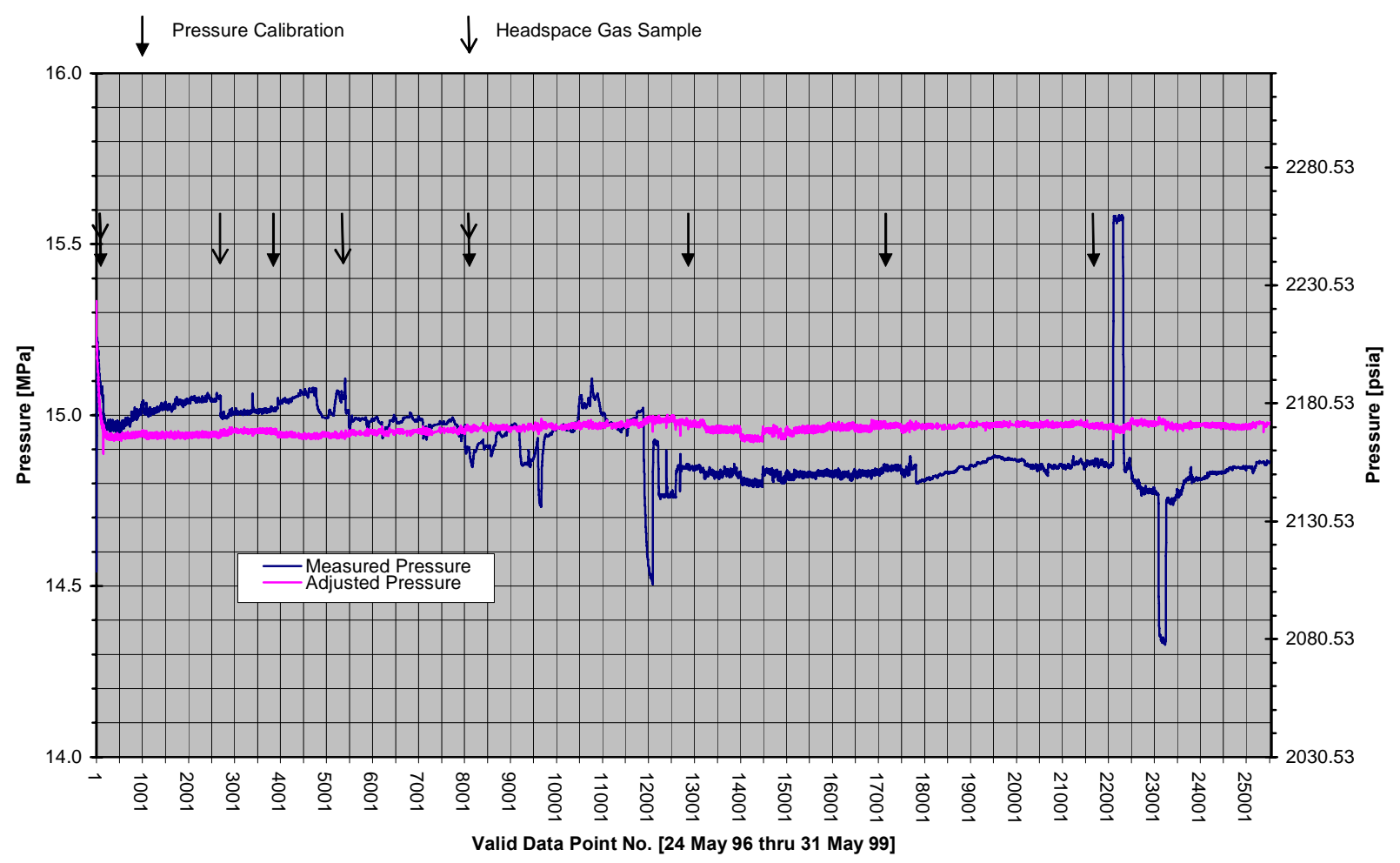

Fig. 24B. Pressures, TC-151 (Ref. Pt. 10001)

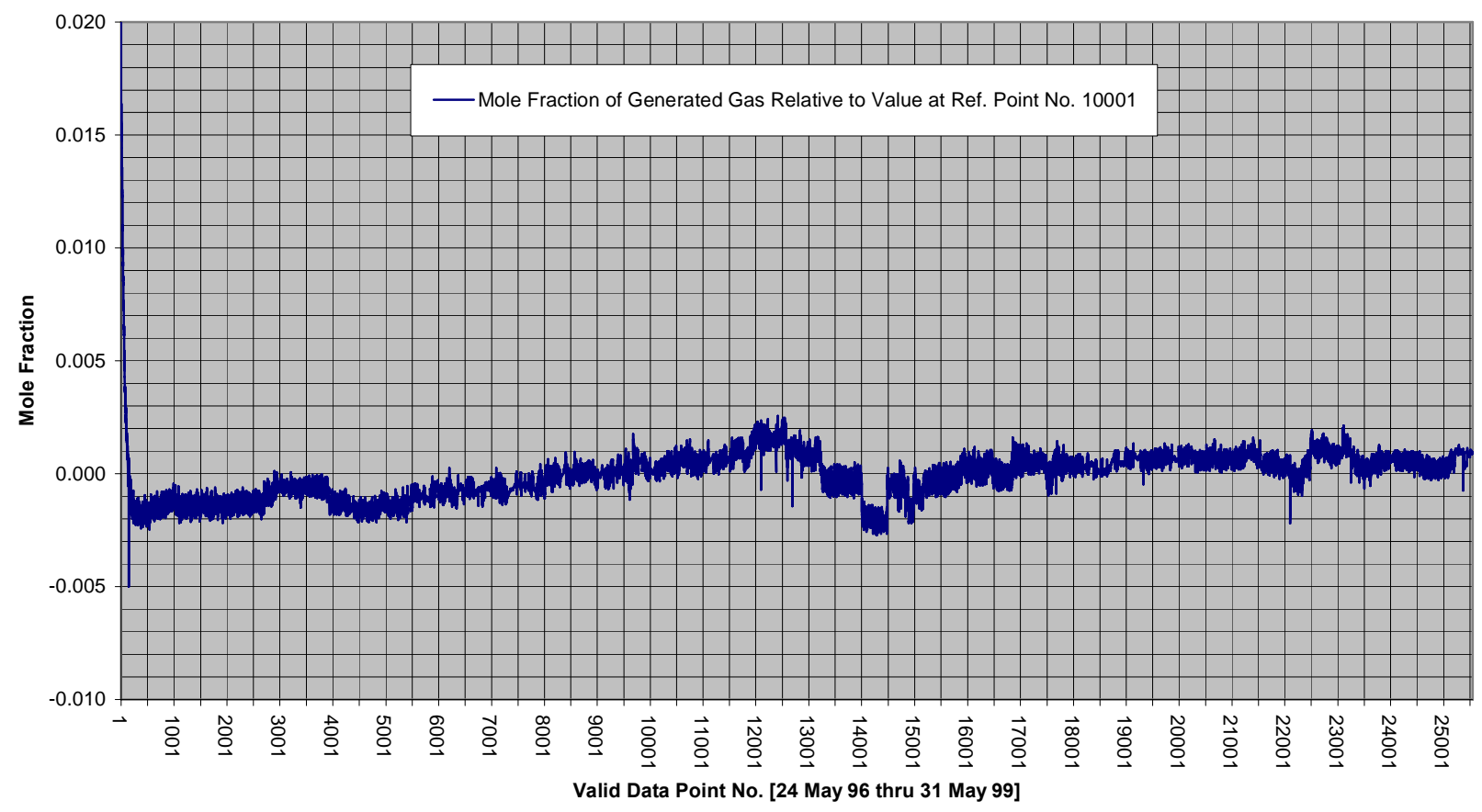

Fig. 24C. Gas Generation in TC-151 


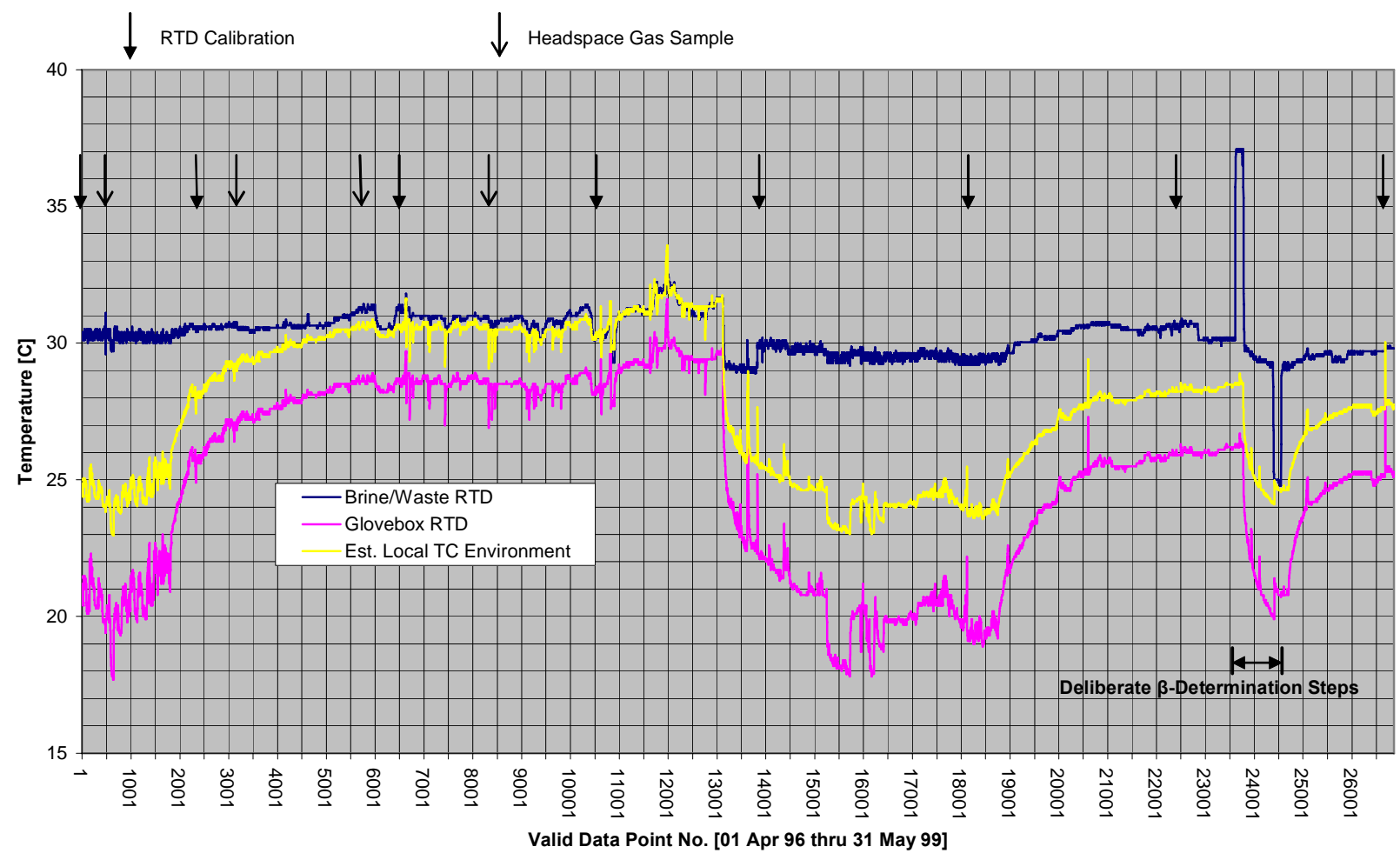

Fig. 25A. Temperatures, TC-160

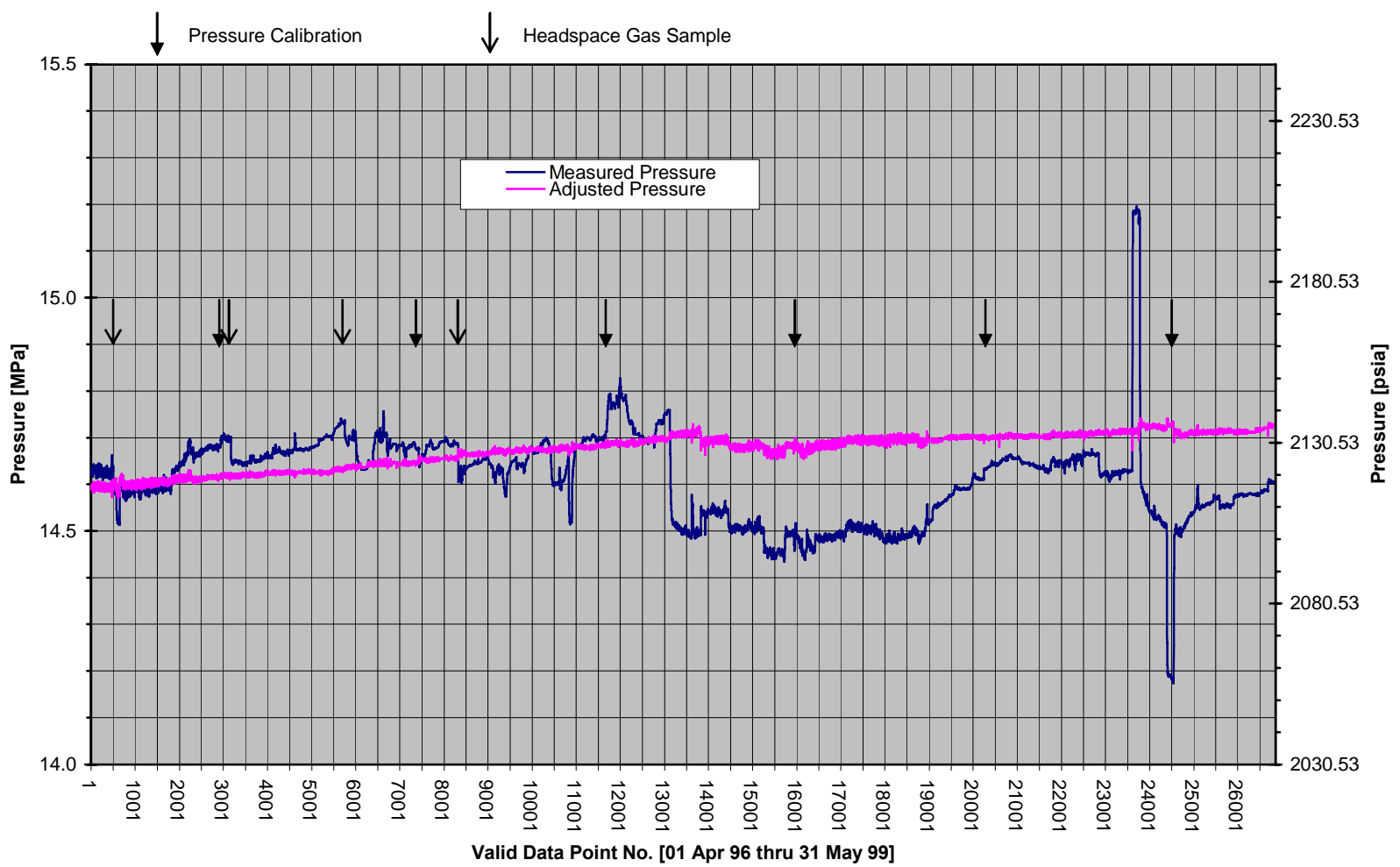

Fig. 25B. Pressures, TC-160 (Ref. Pt. 10001) 


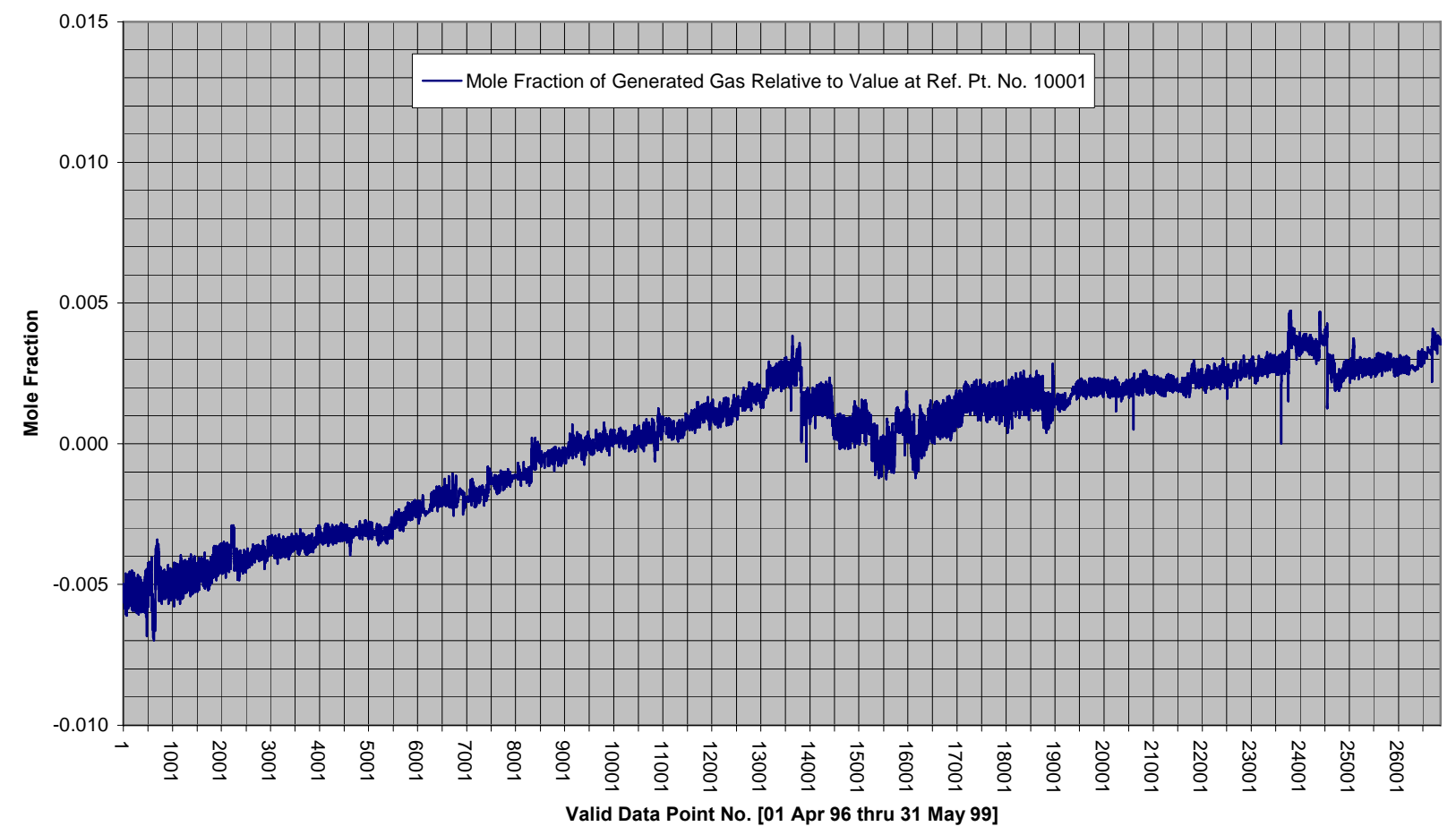

Fig. 25C. Gas Generation in TC-160

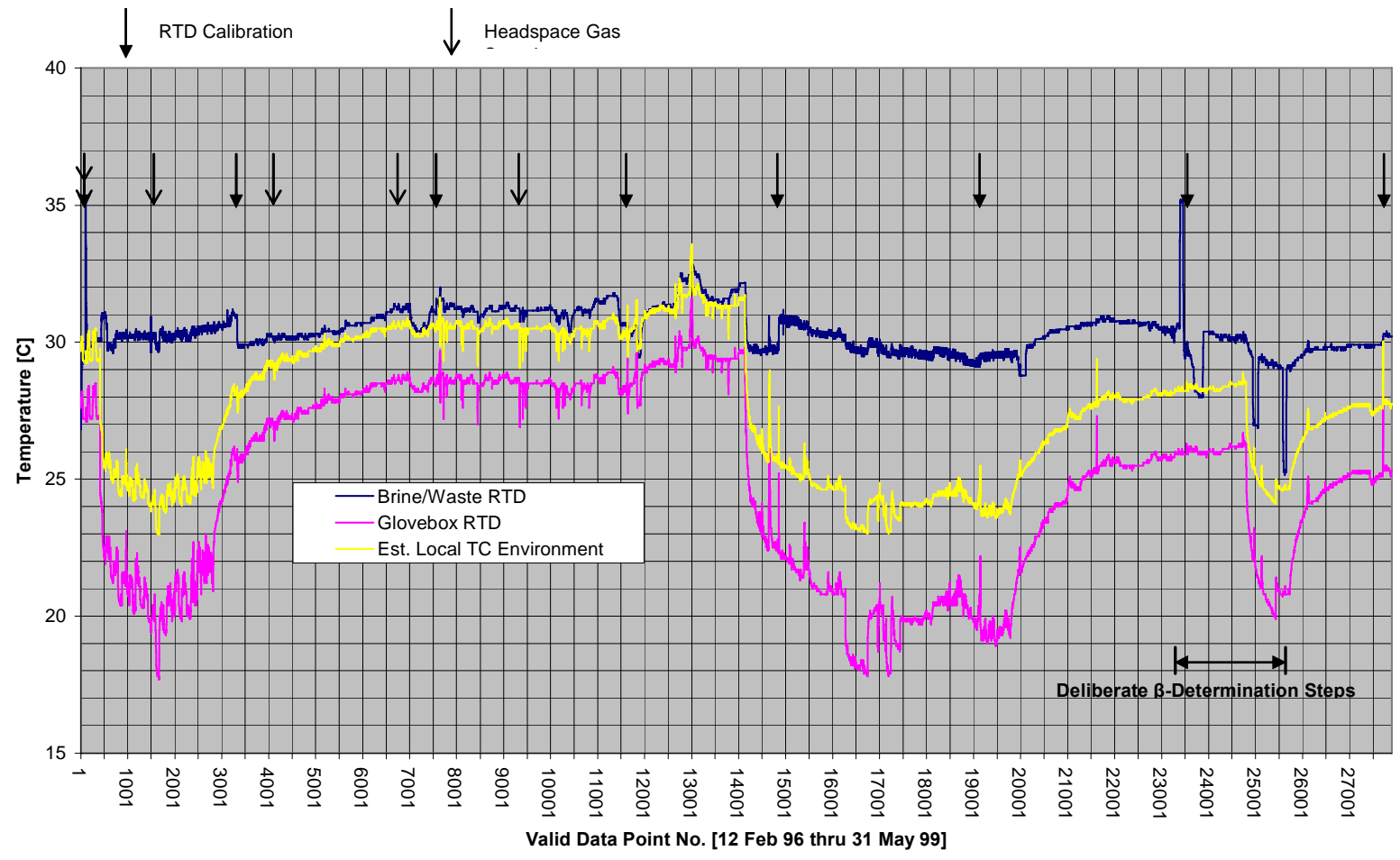

Fig. 26A. Temperature, TC-161 


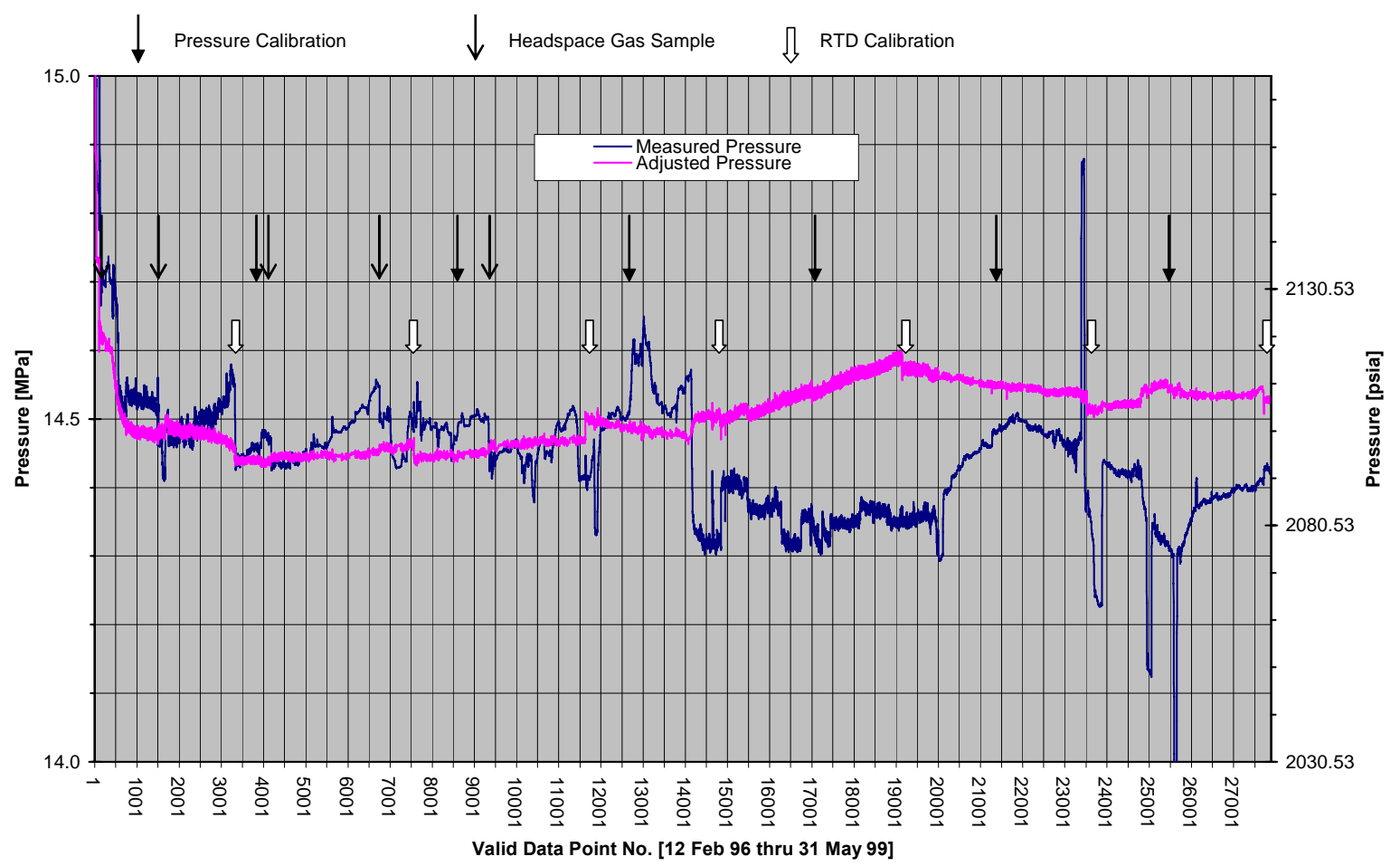

Fig. 26B. Pressures, TC-161 (Ref. Point No. 10001)

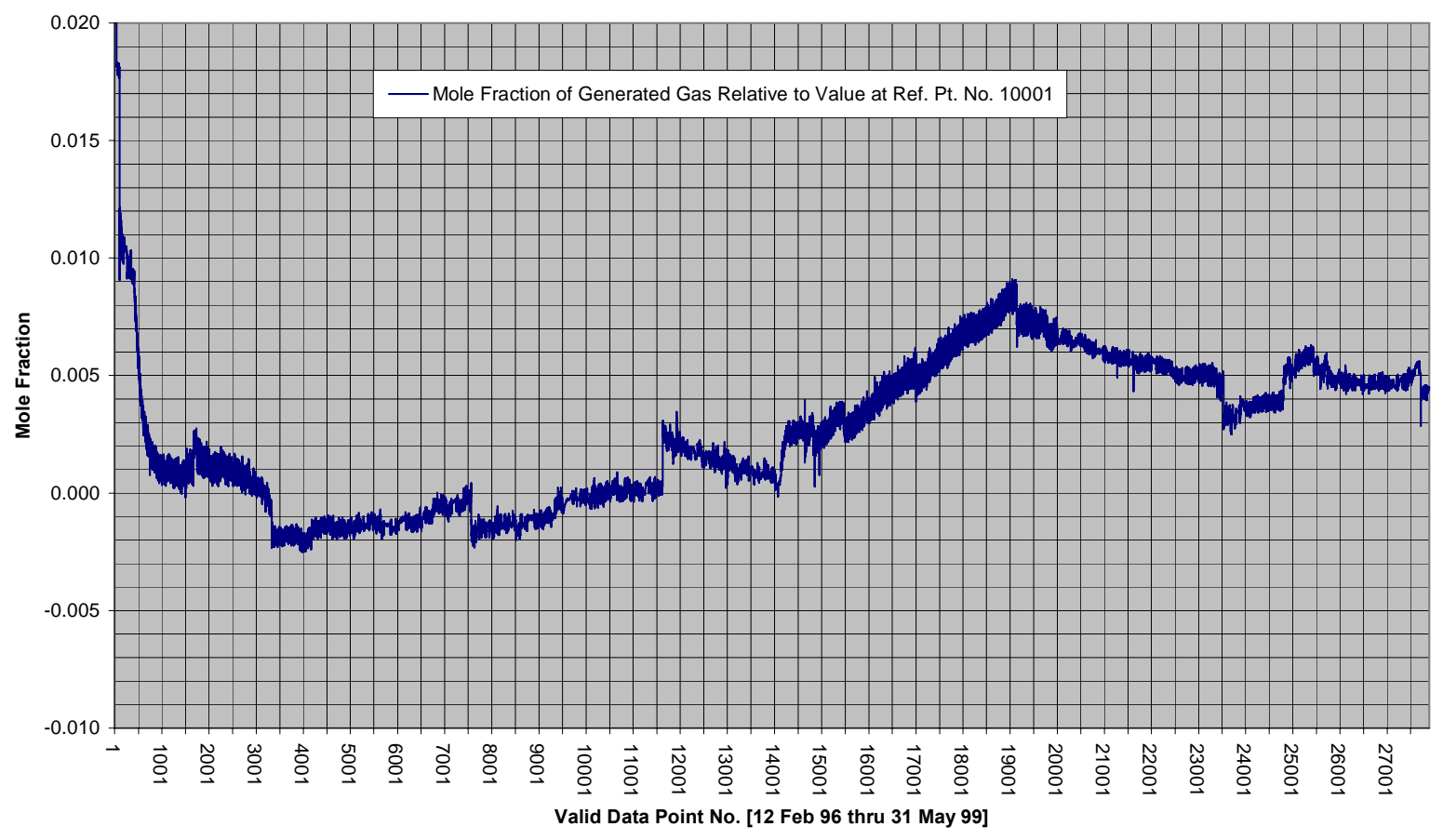

Fig. 26C. Gas Generation in TC-161 


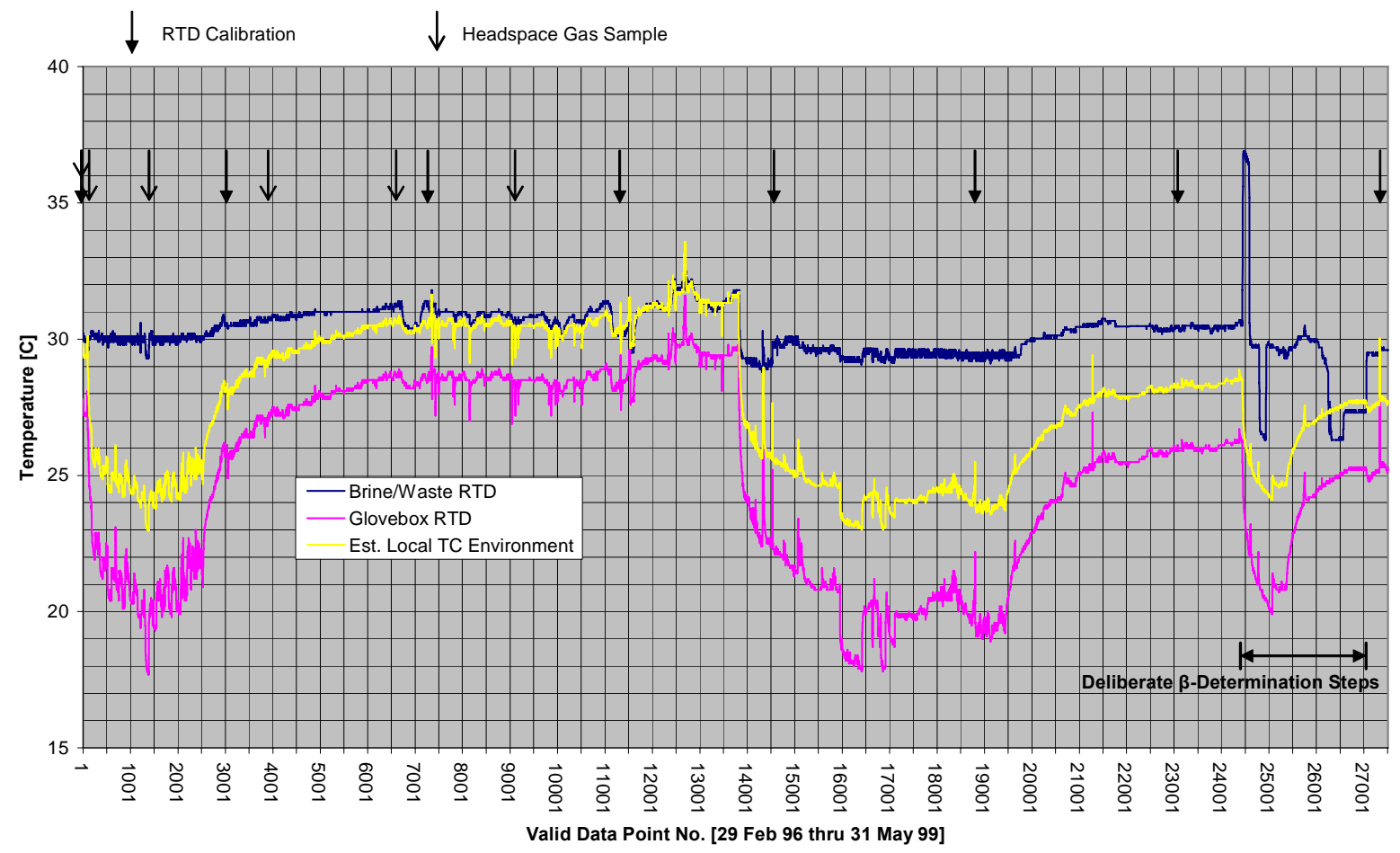

Fig. 27A. Temperatures, TC-162

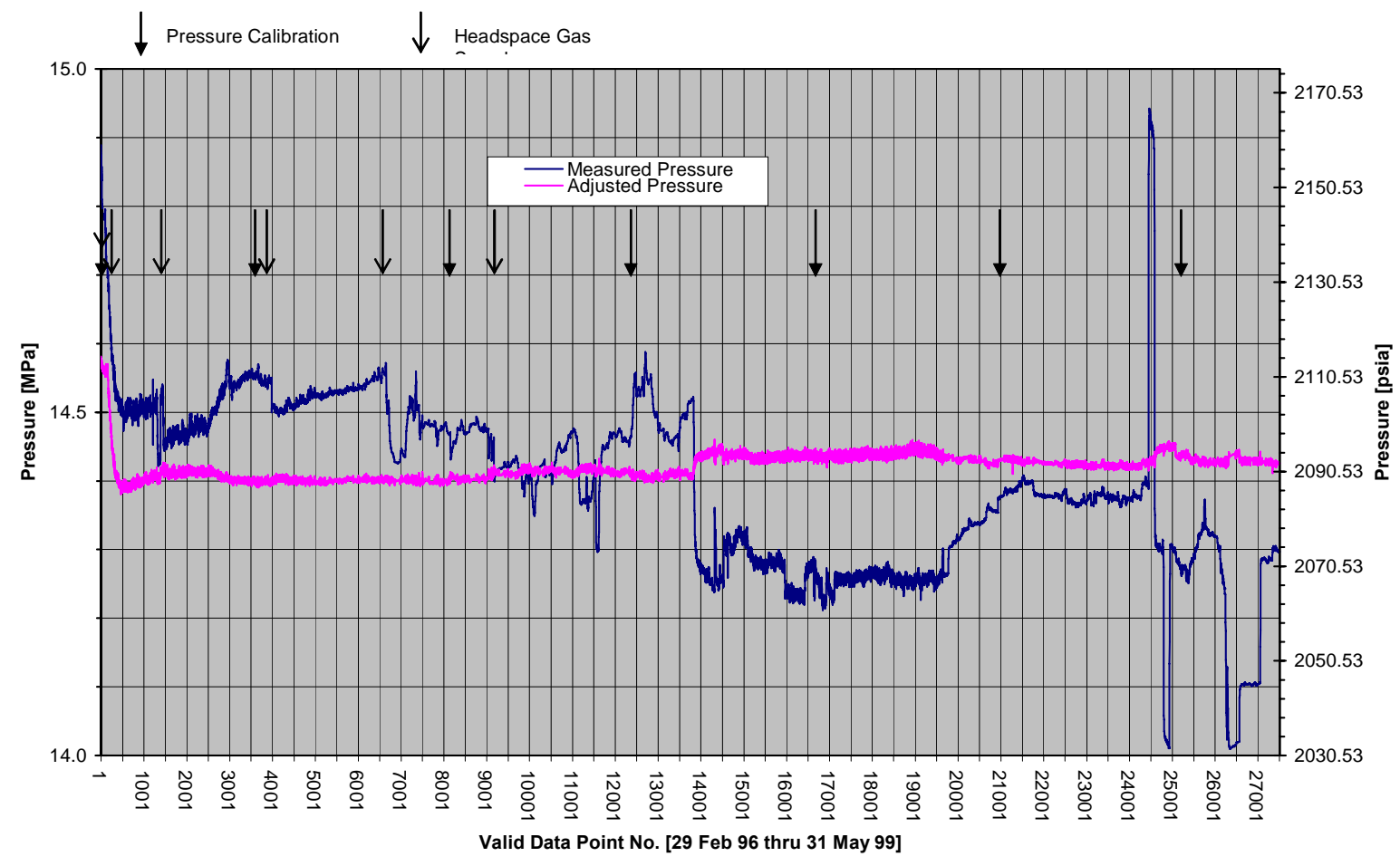

Fig. 27B. Pressures, TC-162 (Ref. Point No. 10001) 


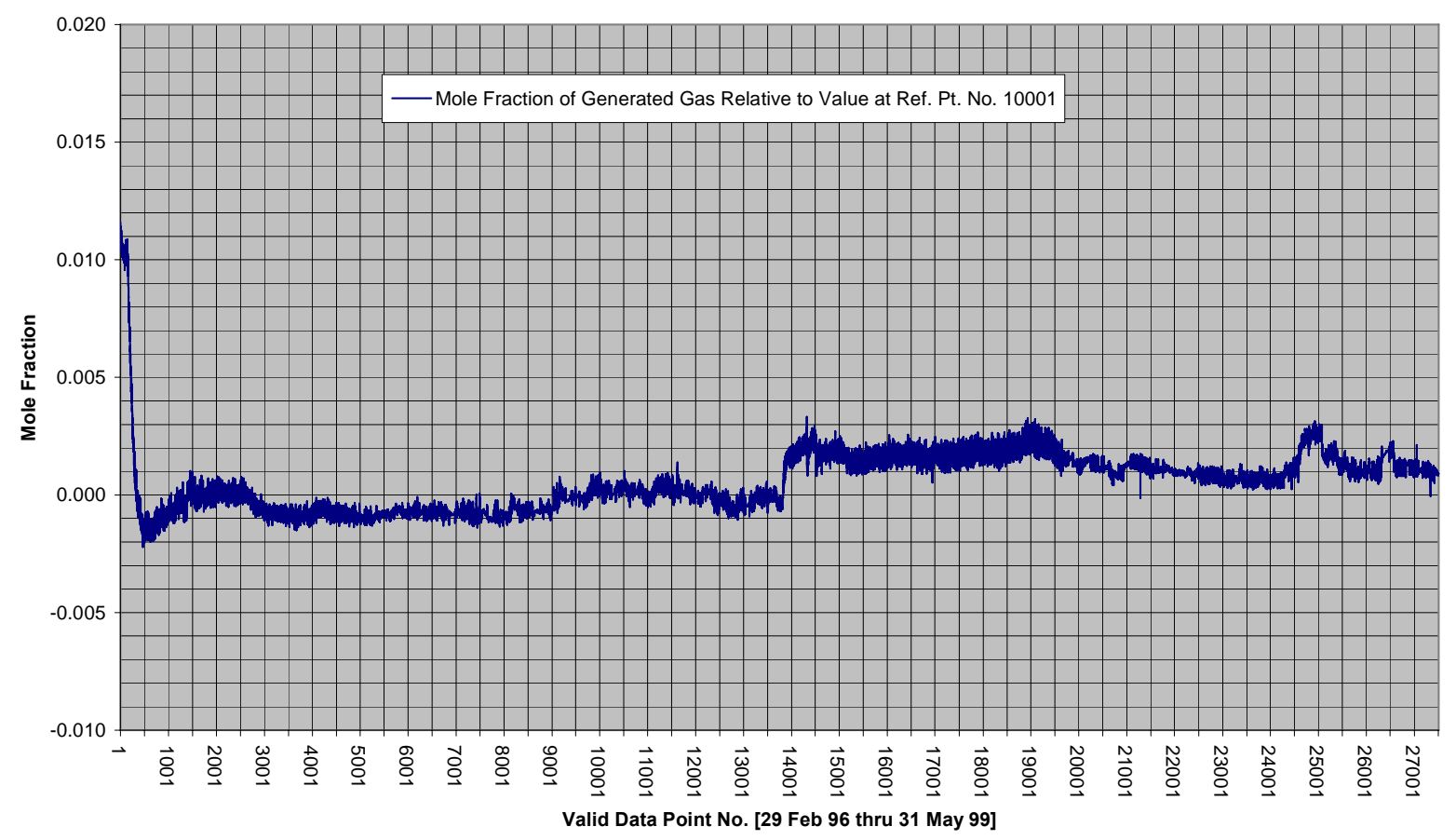

Fig. 27C. Gas Generation in TC-162

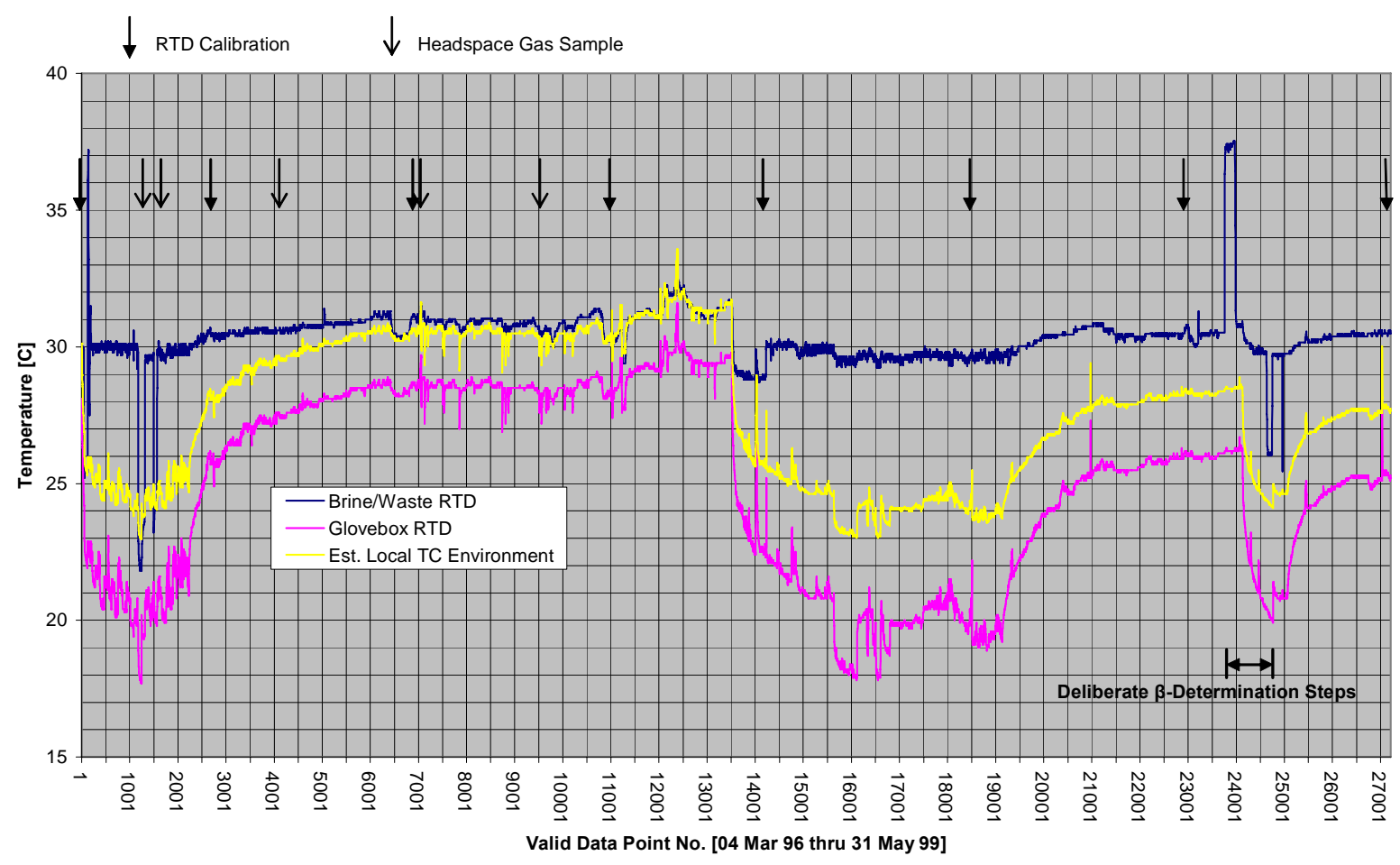

Fig. 28A. Temperatures, TC-163 


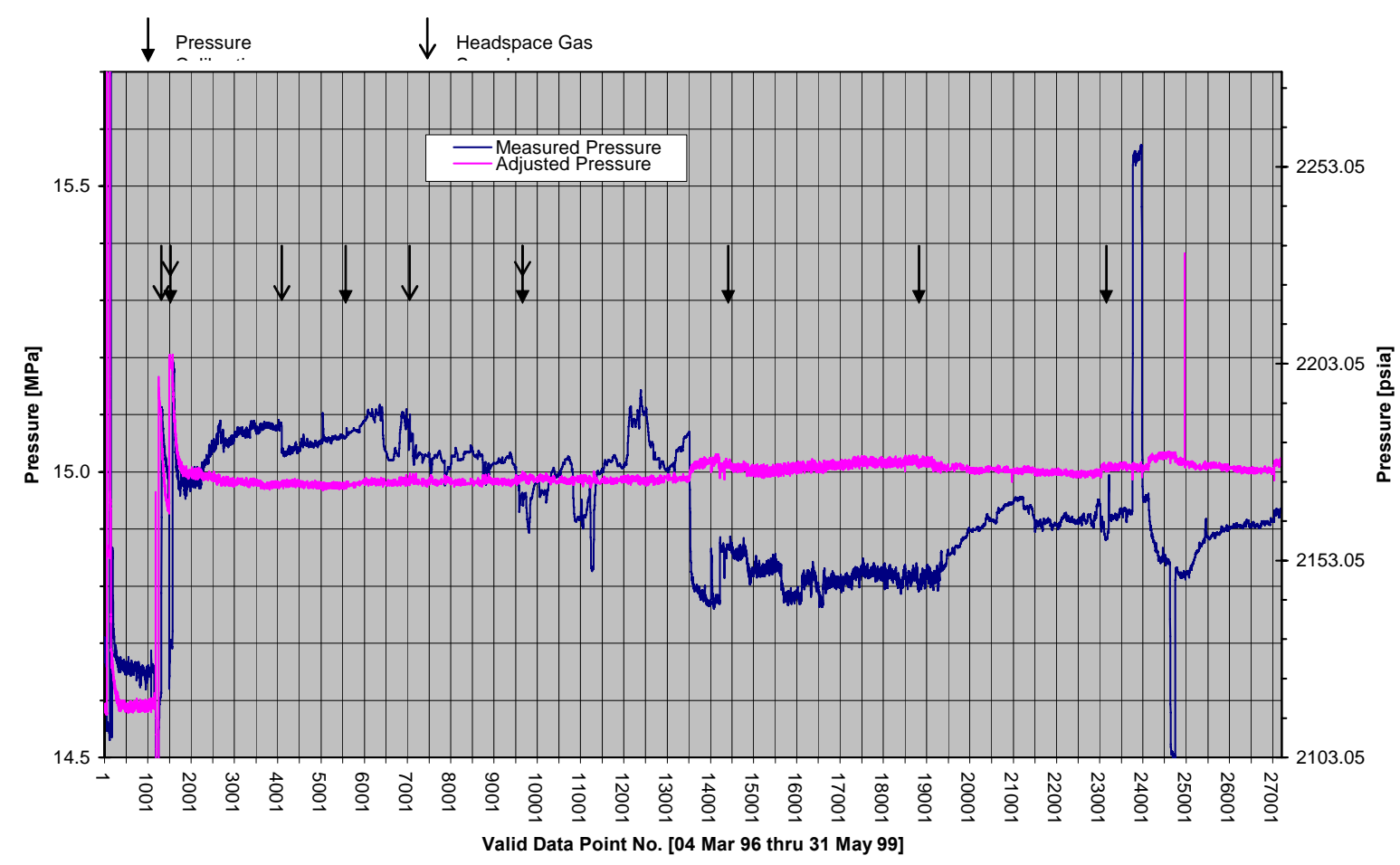

Fig. 28B. Pressures, TC-163 (Ref. Point No. 10001)

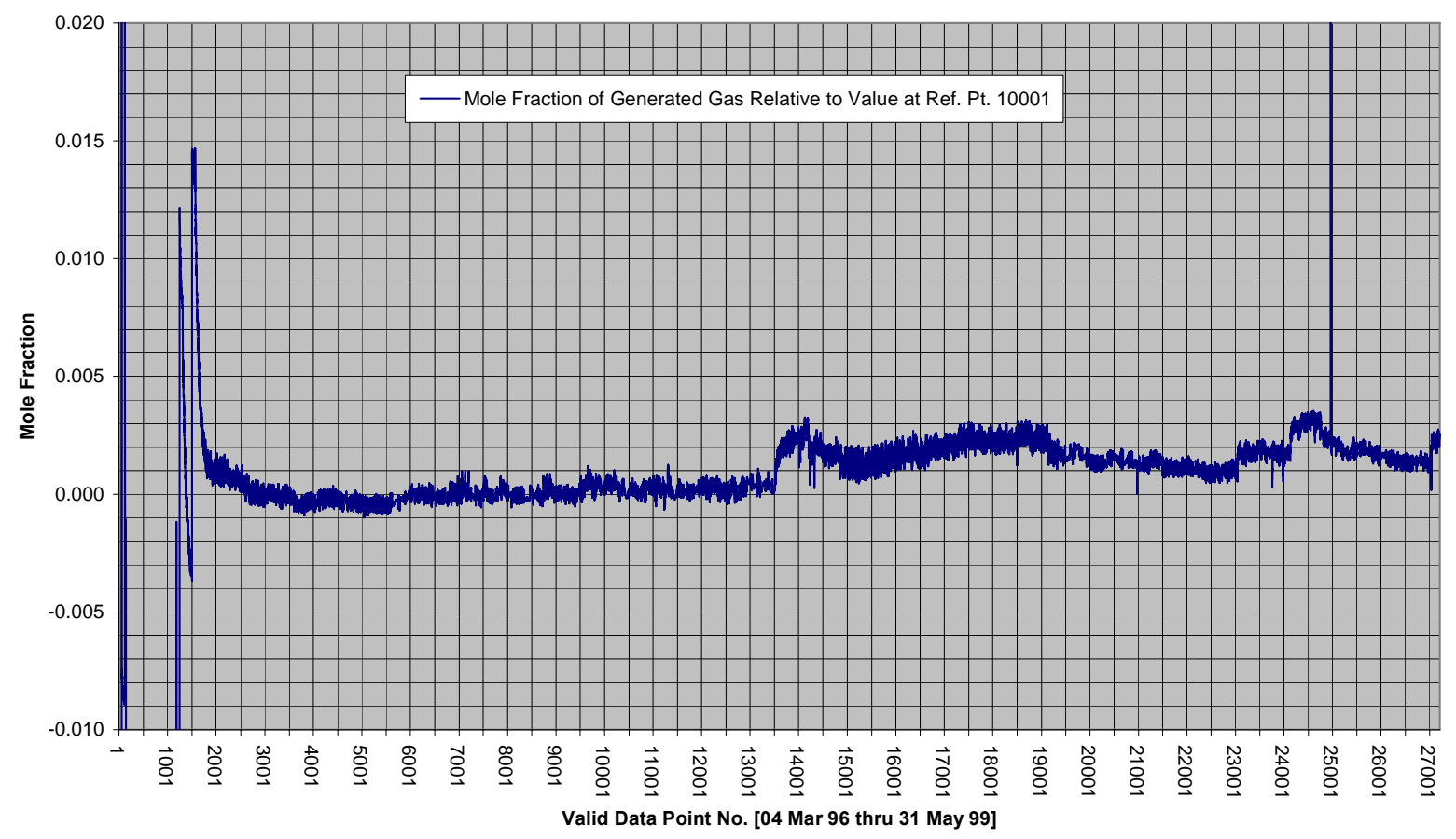

Fig. 28C. Generated Gas, TC-163 


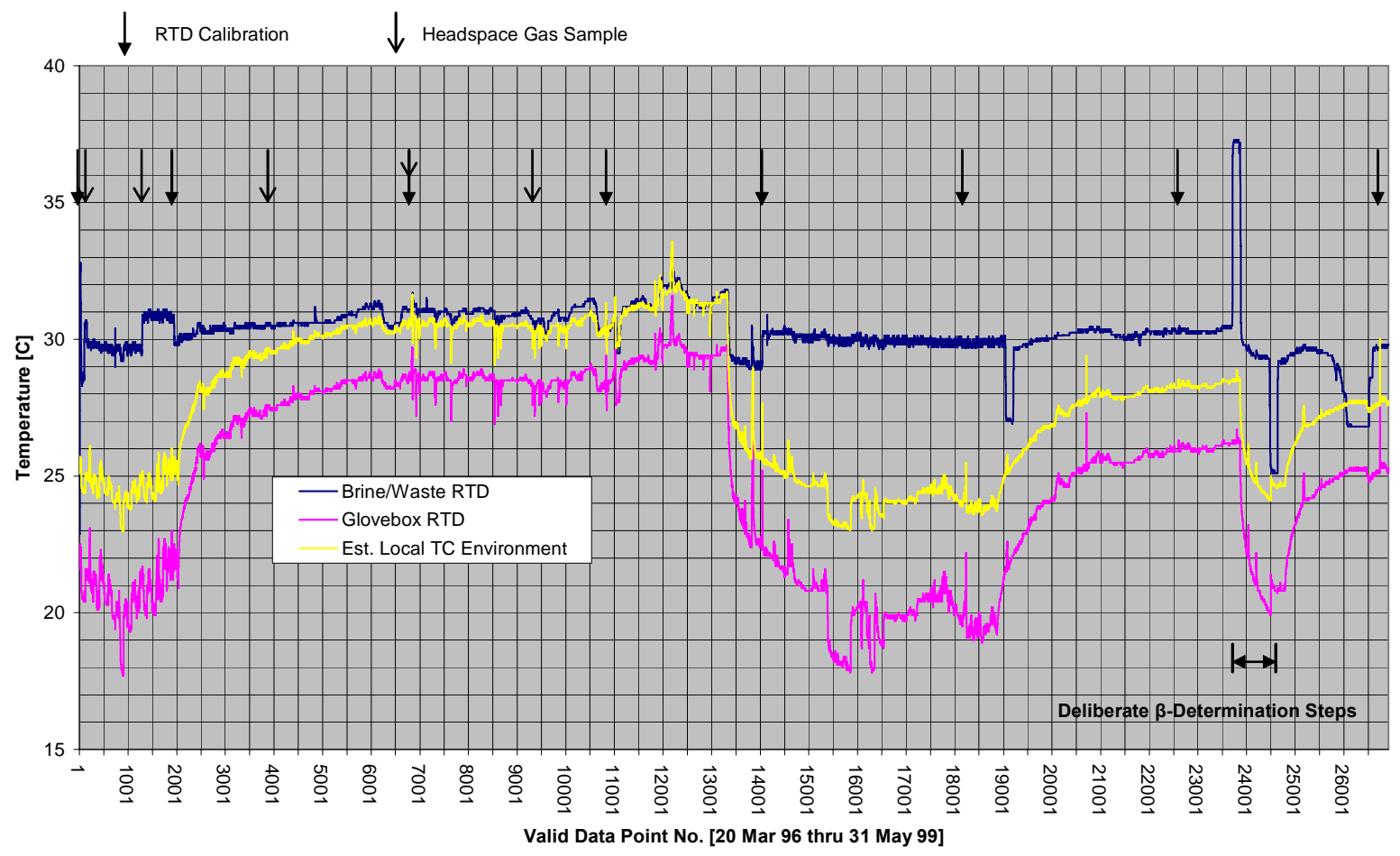

Fig. 29A. Temperature, TC-164

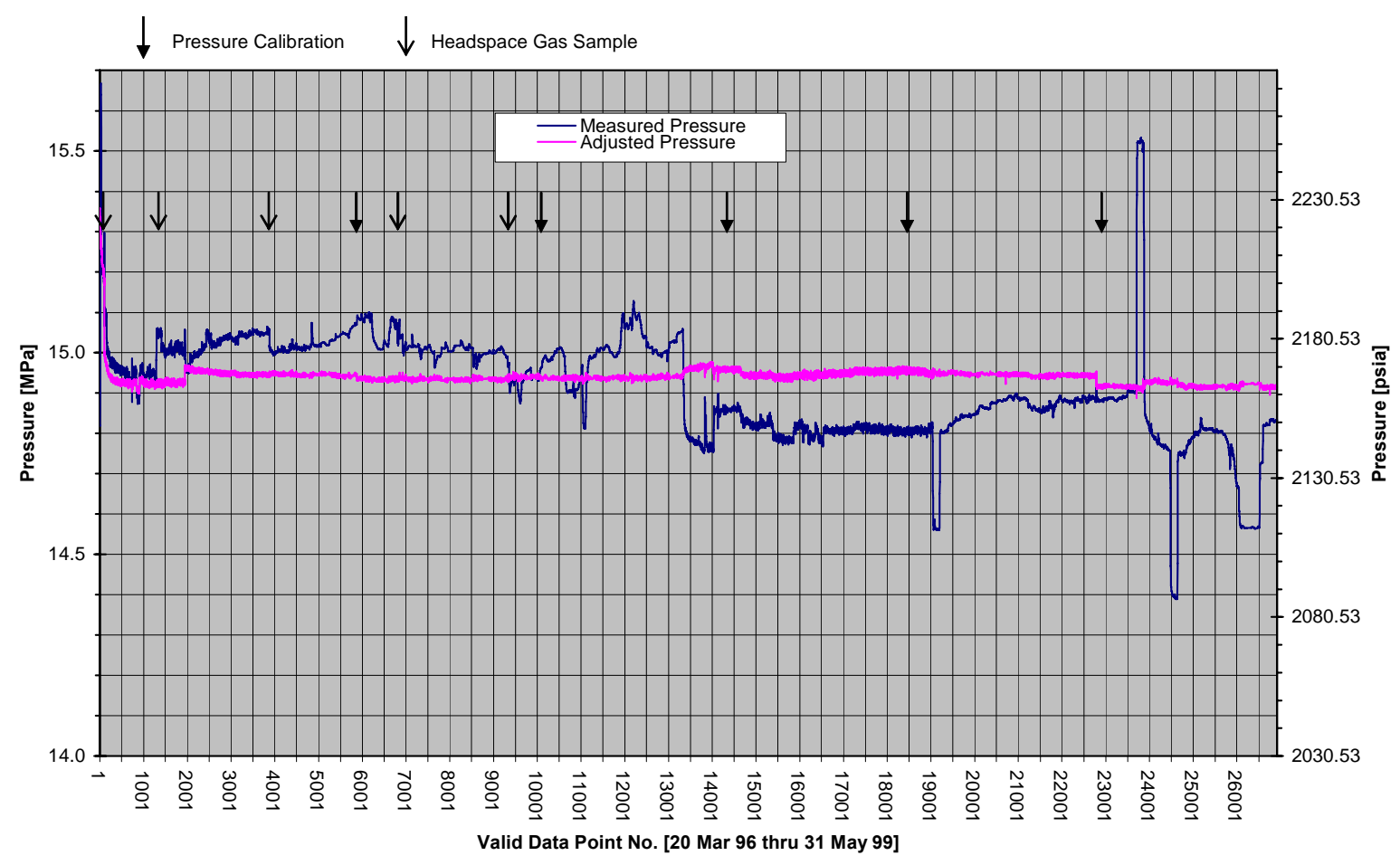

Fig. 29B. Pressure, TC-164 (Ref. Point No. 10001) 


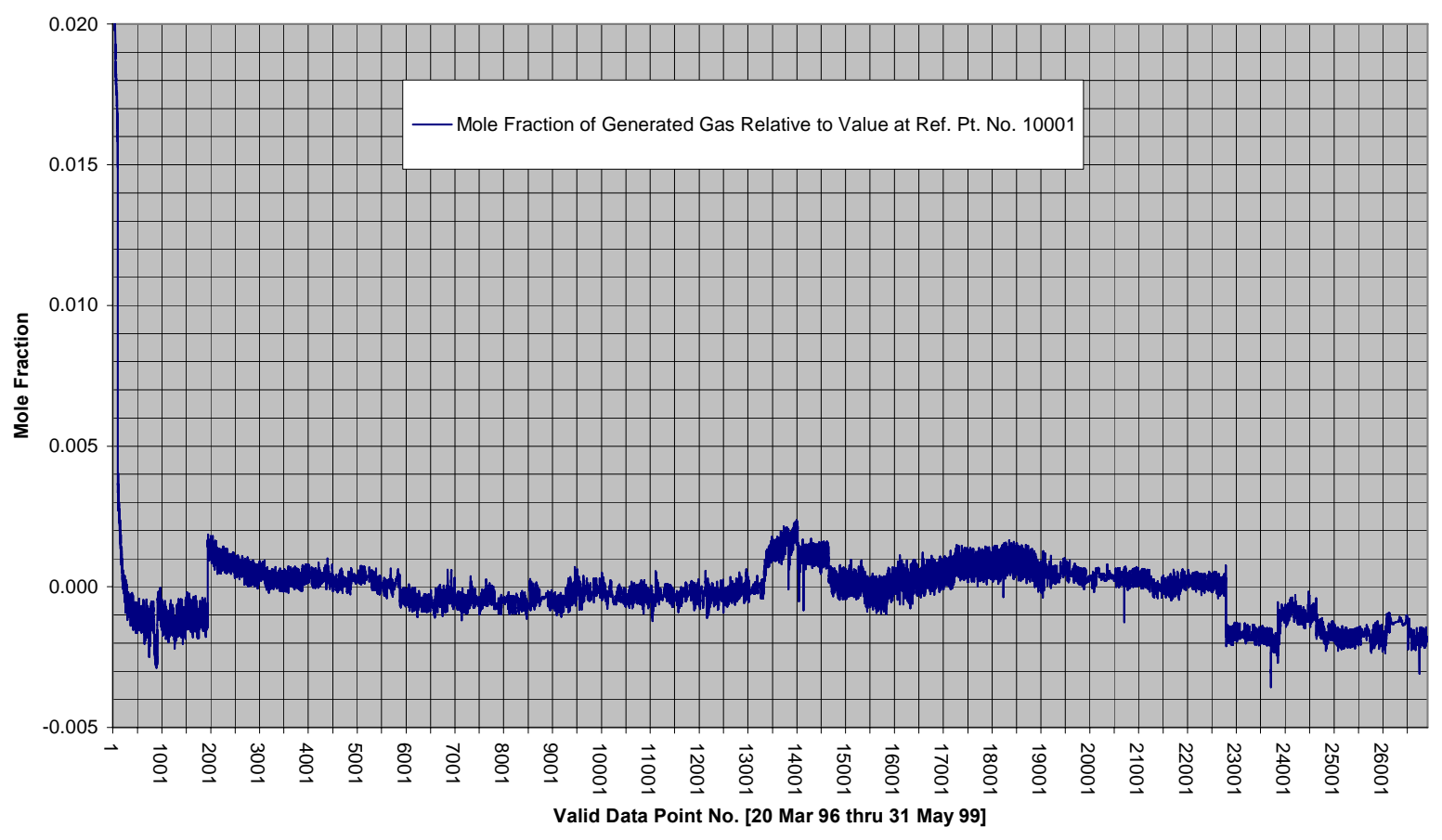

Fig. 29C. Gas Generation in TC-164

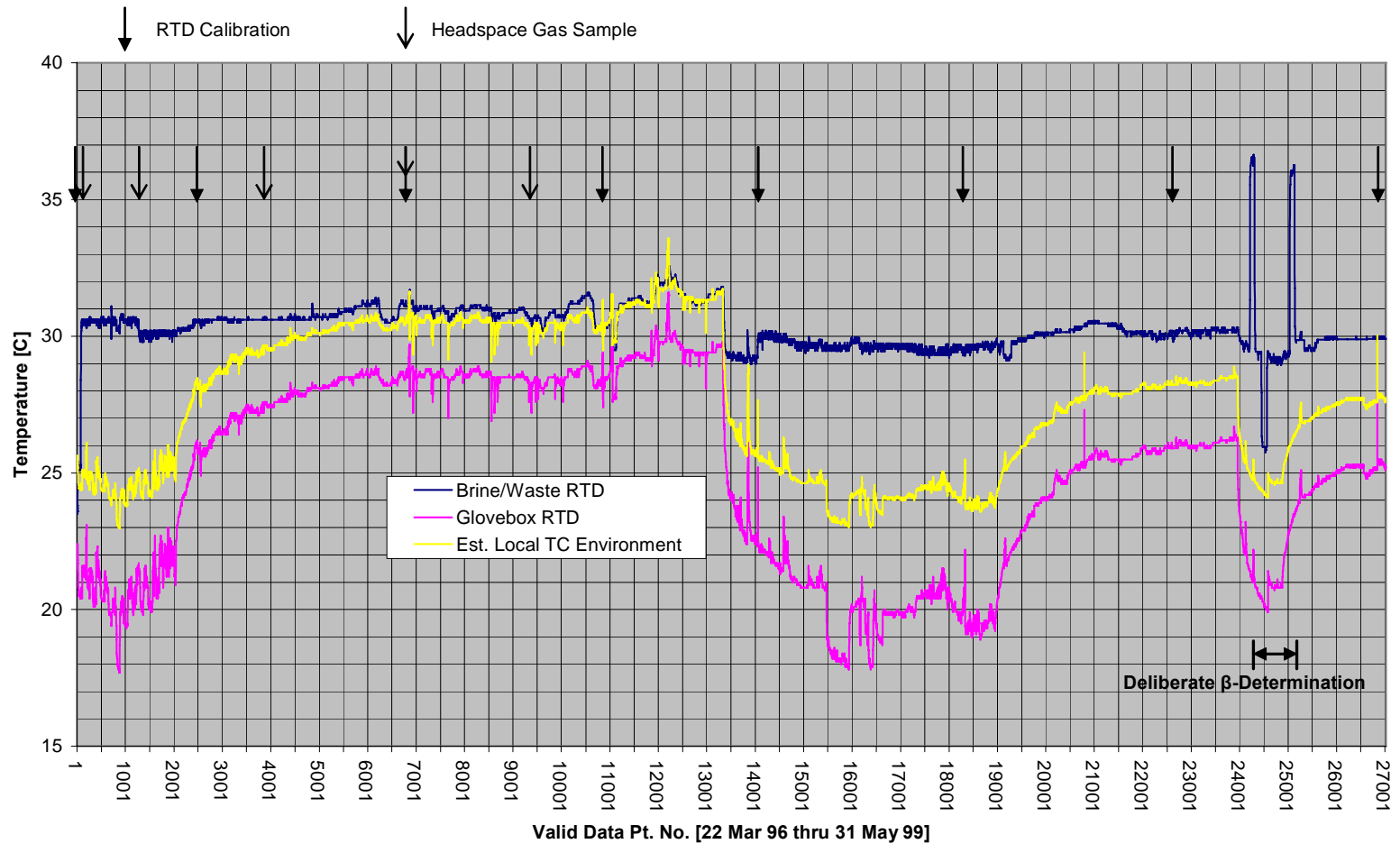

Fig. 30A. Temperatures, TC-165 


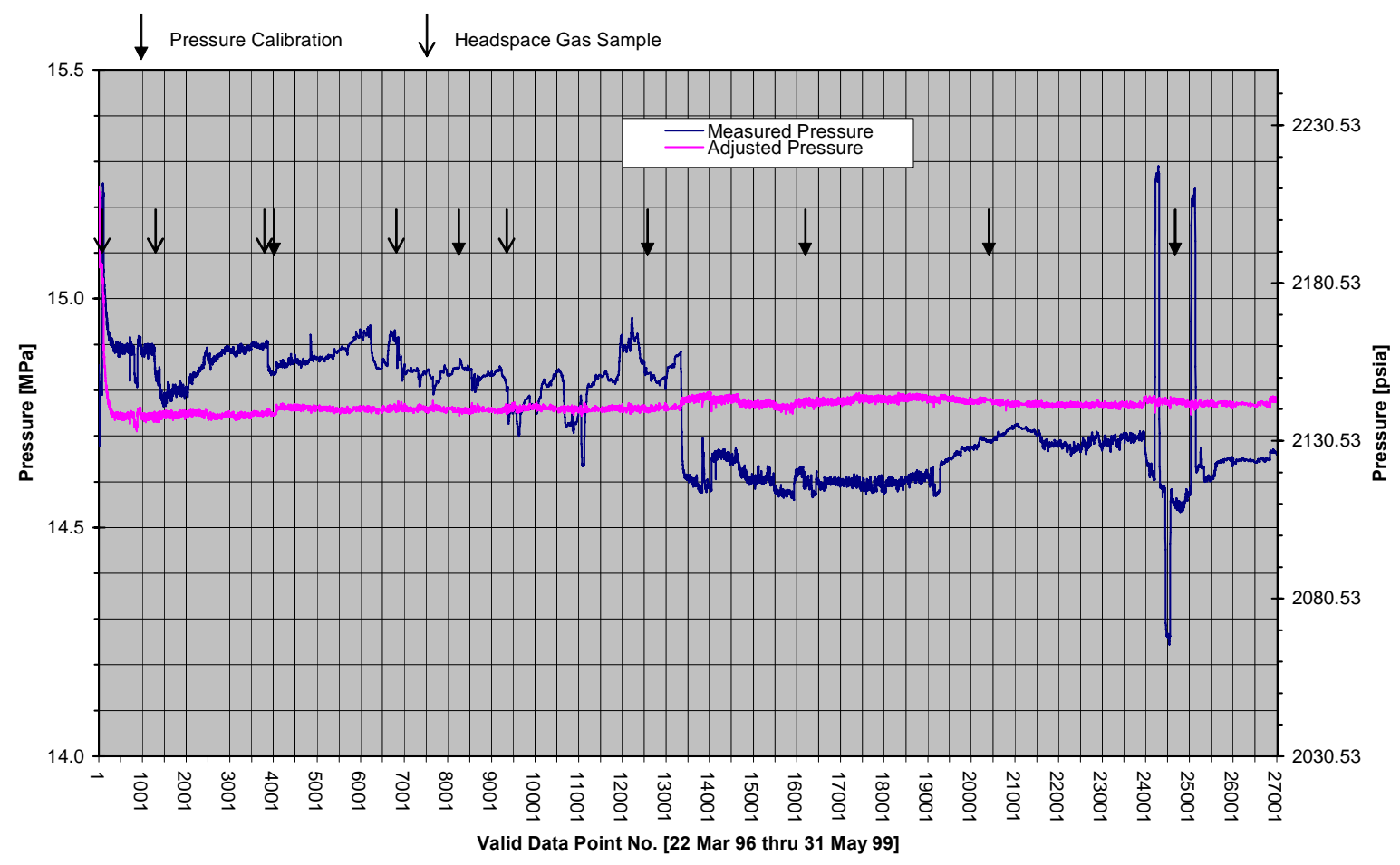

Fig. 30B. Pressures, TC-165 (Ref. Point No. 10001)

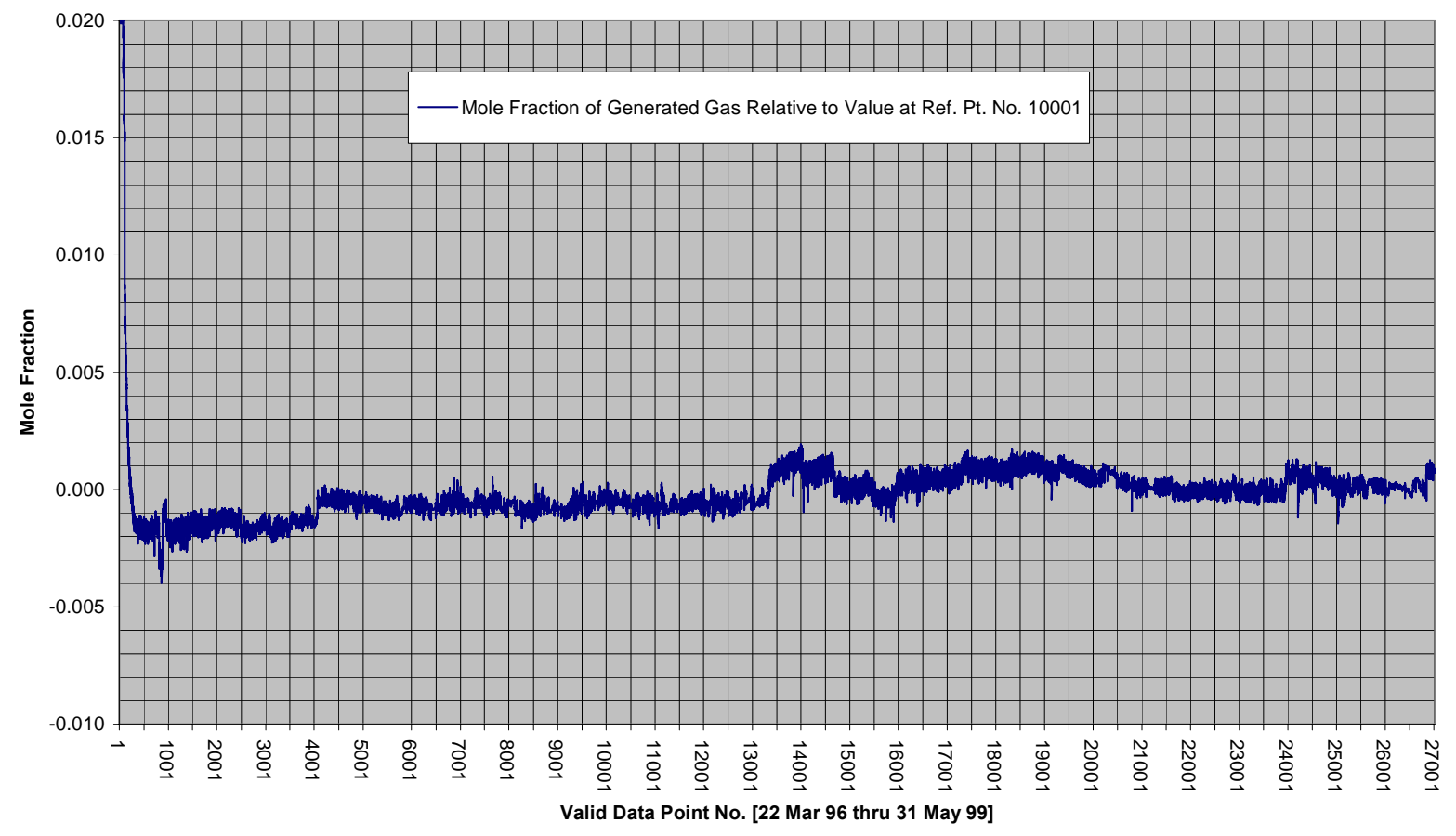

Fig. 30C. Gas Generation, TC-165 


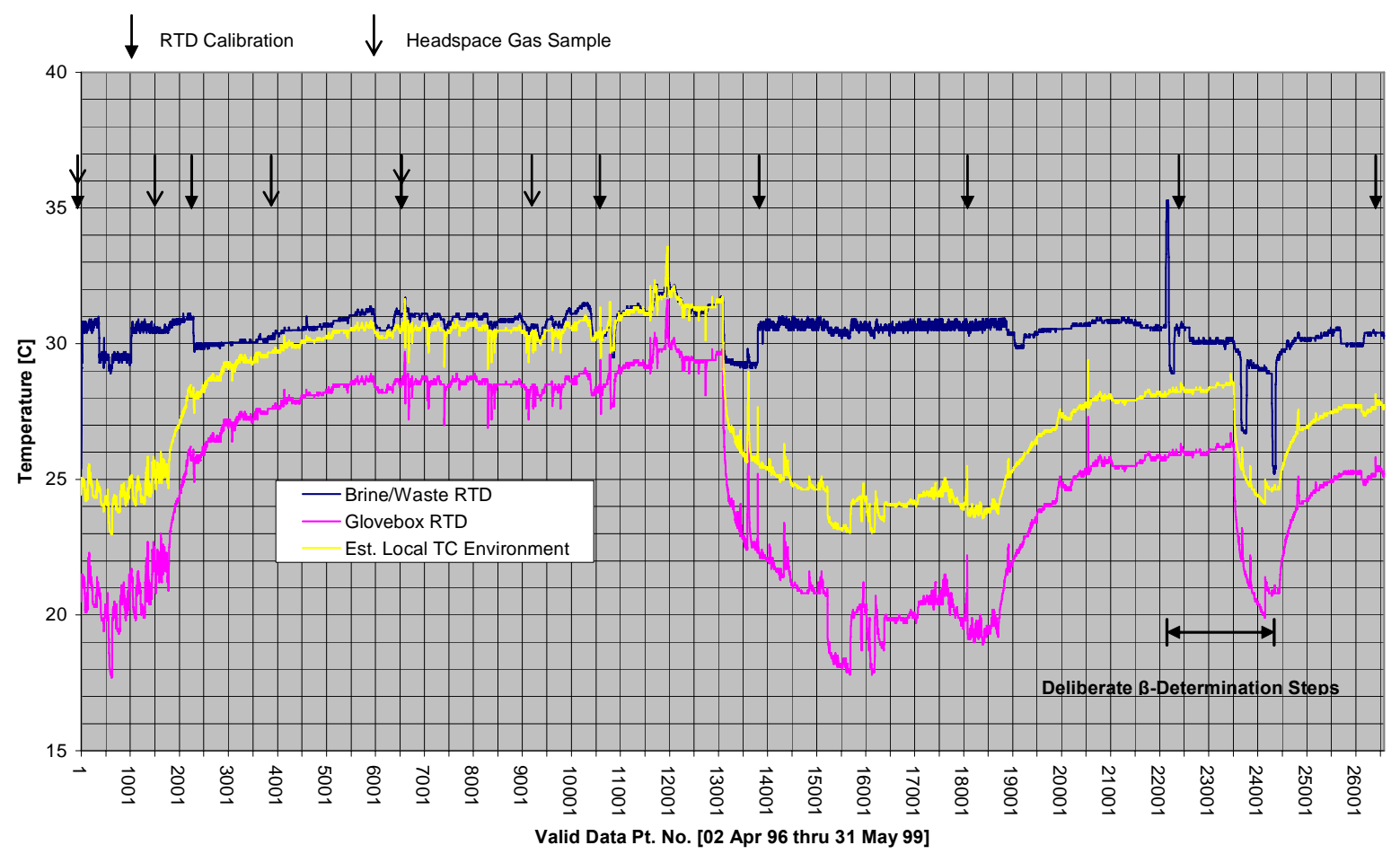

Fig. 31A. Temperatures, TC-166

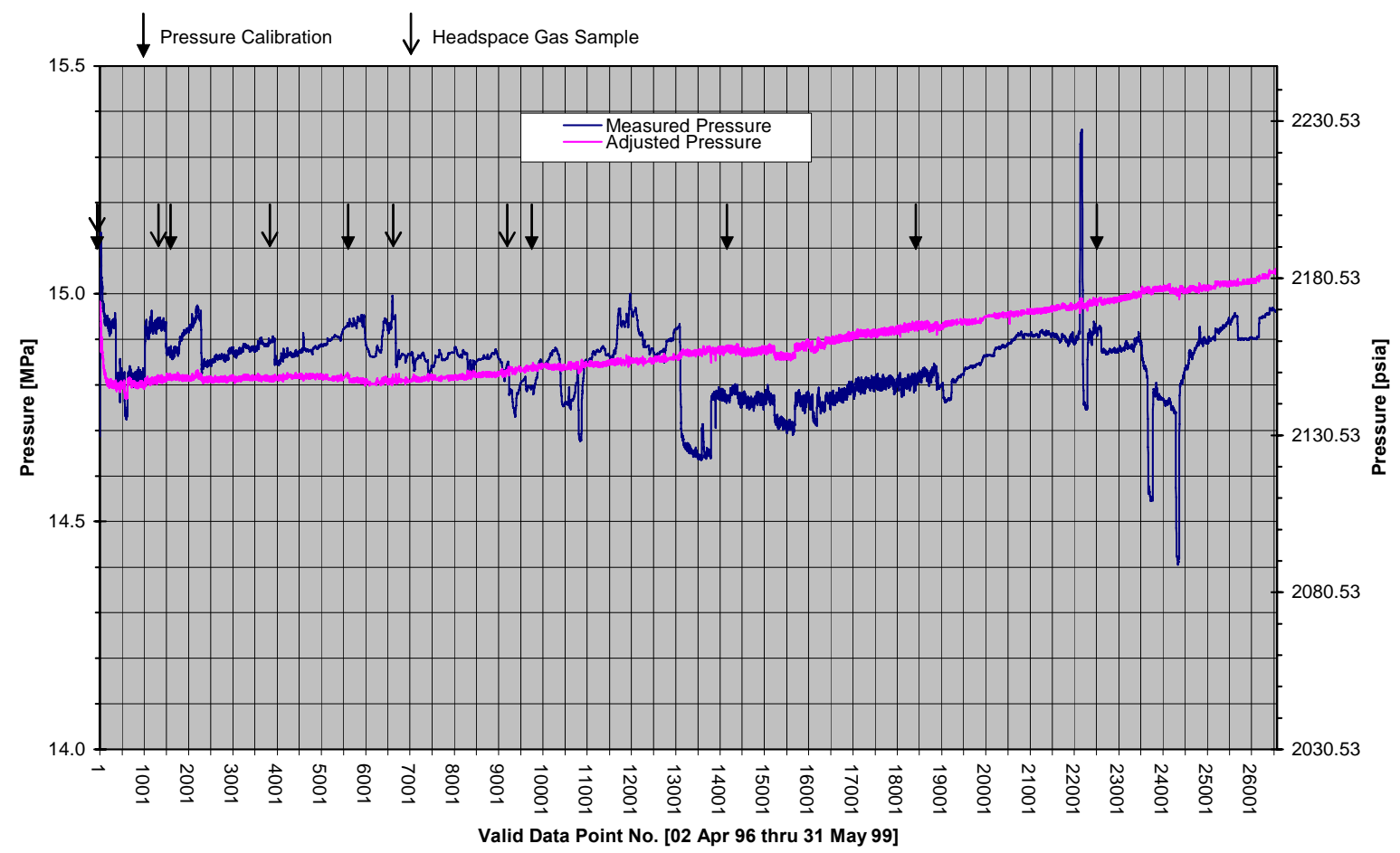

Fig. 31B. Pressures, TC-166 (Ref. Point No. 10001) 


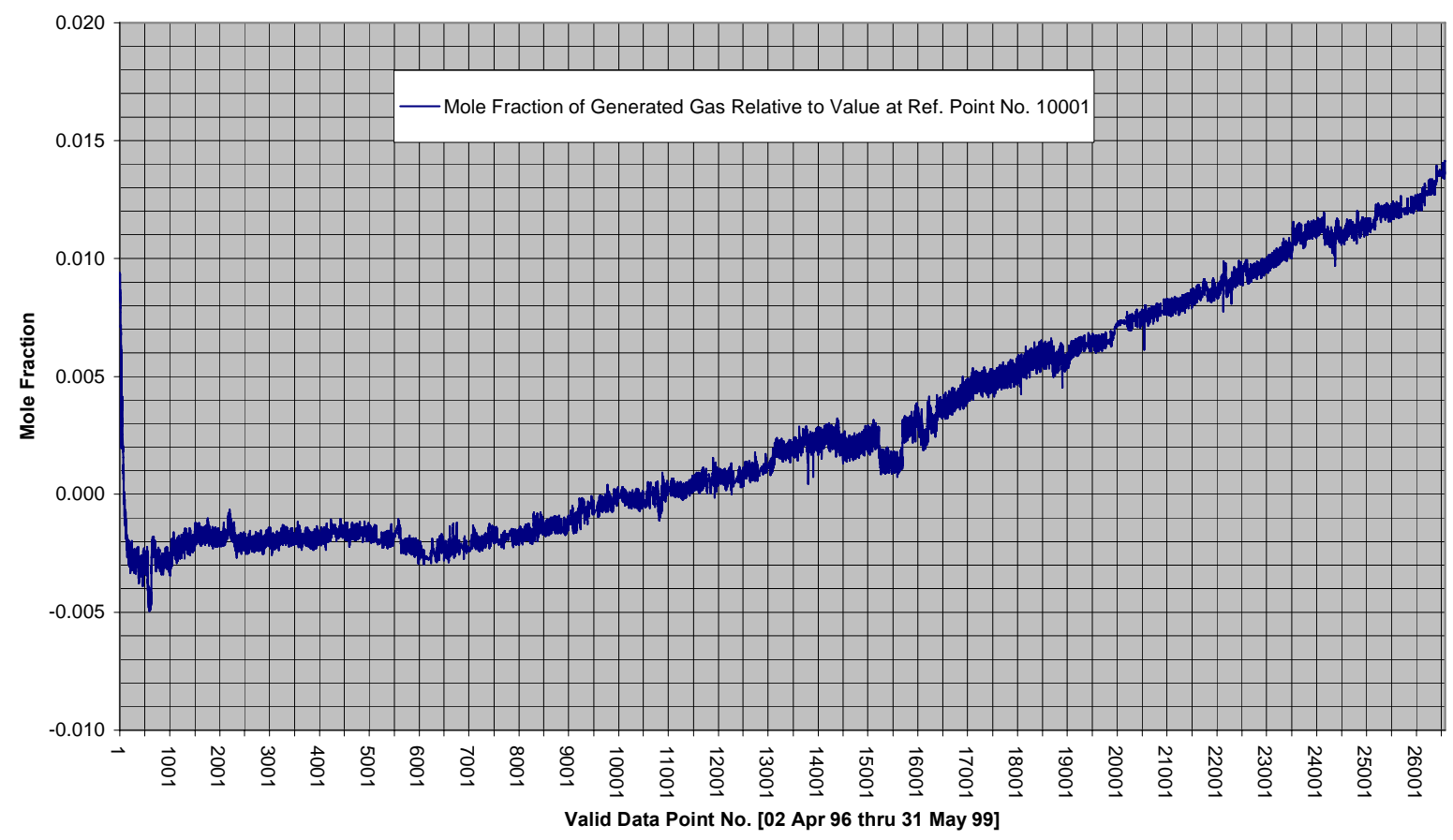

Fig. 31C. Gas Generation, TC-166

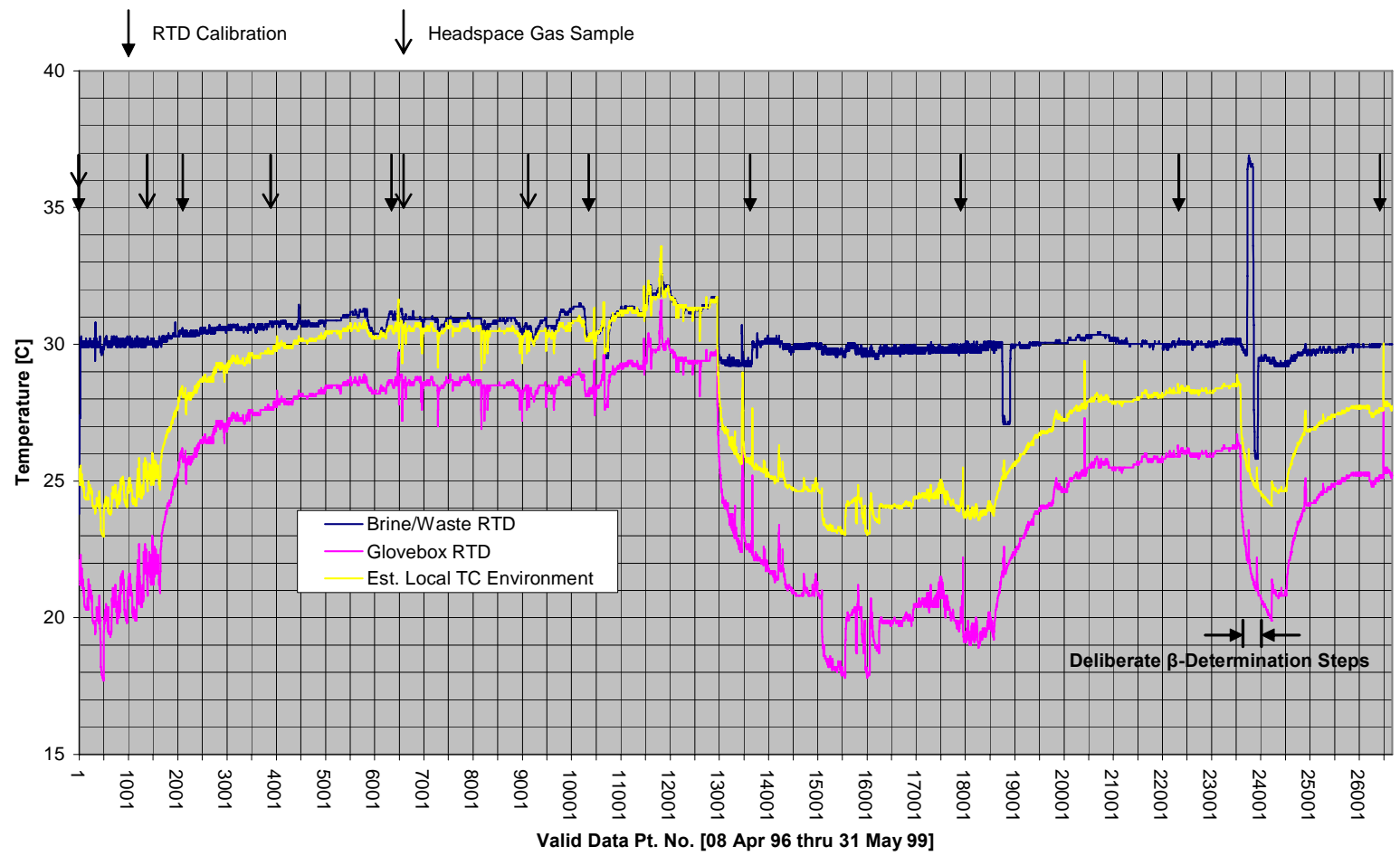

Fig. 32A. Temperatures, TC-167 


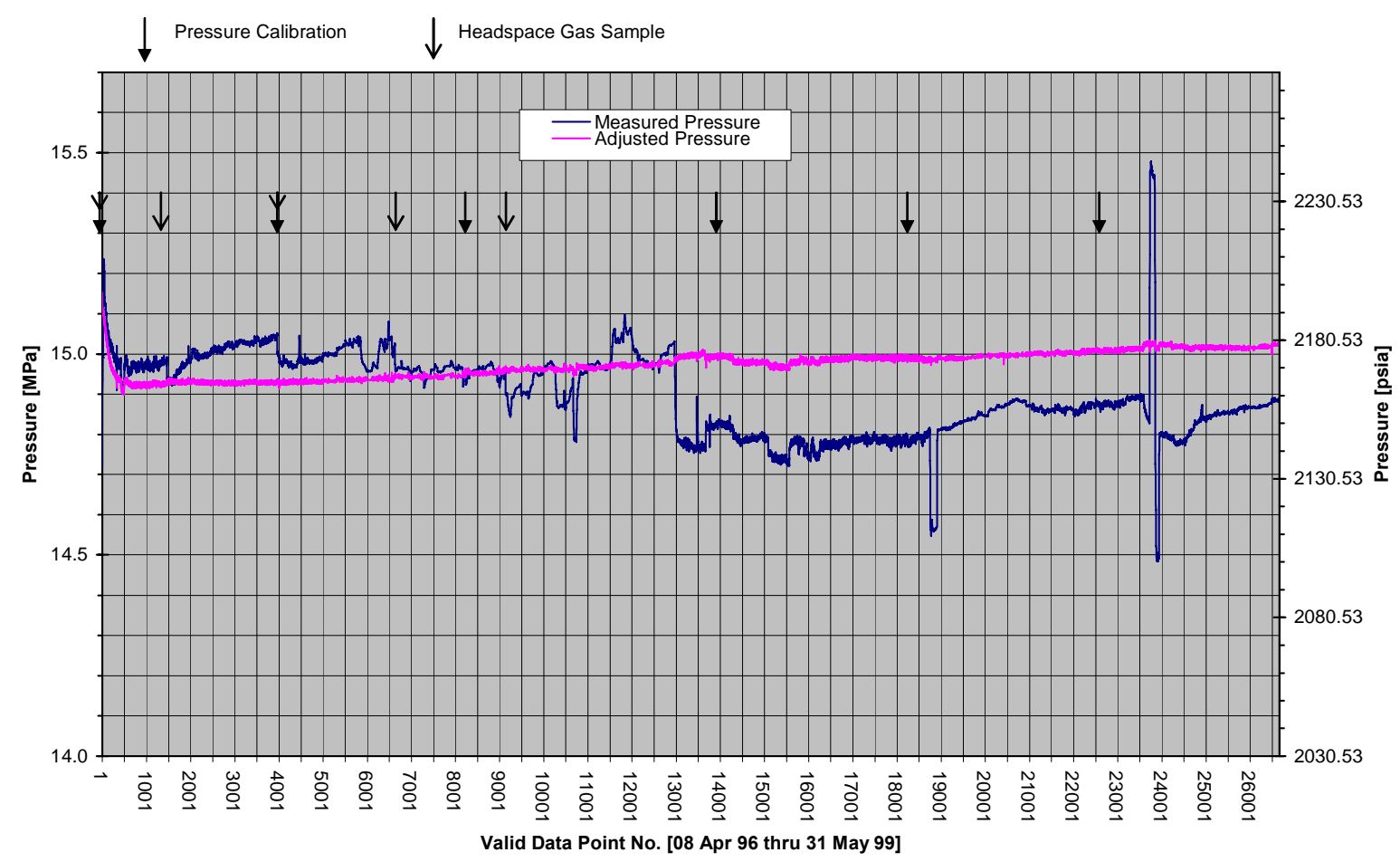

Fig. 32B. Pressures, TC-167 (Ref. Point No. 10001)

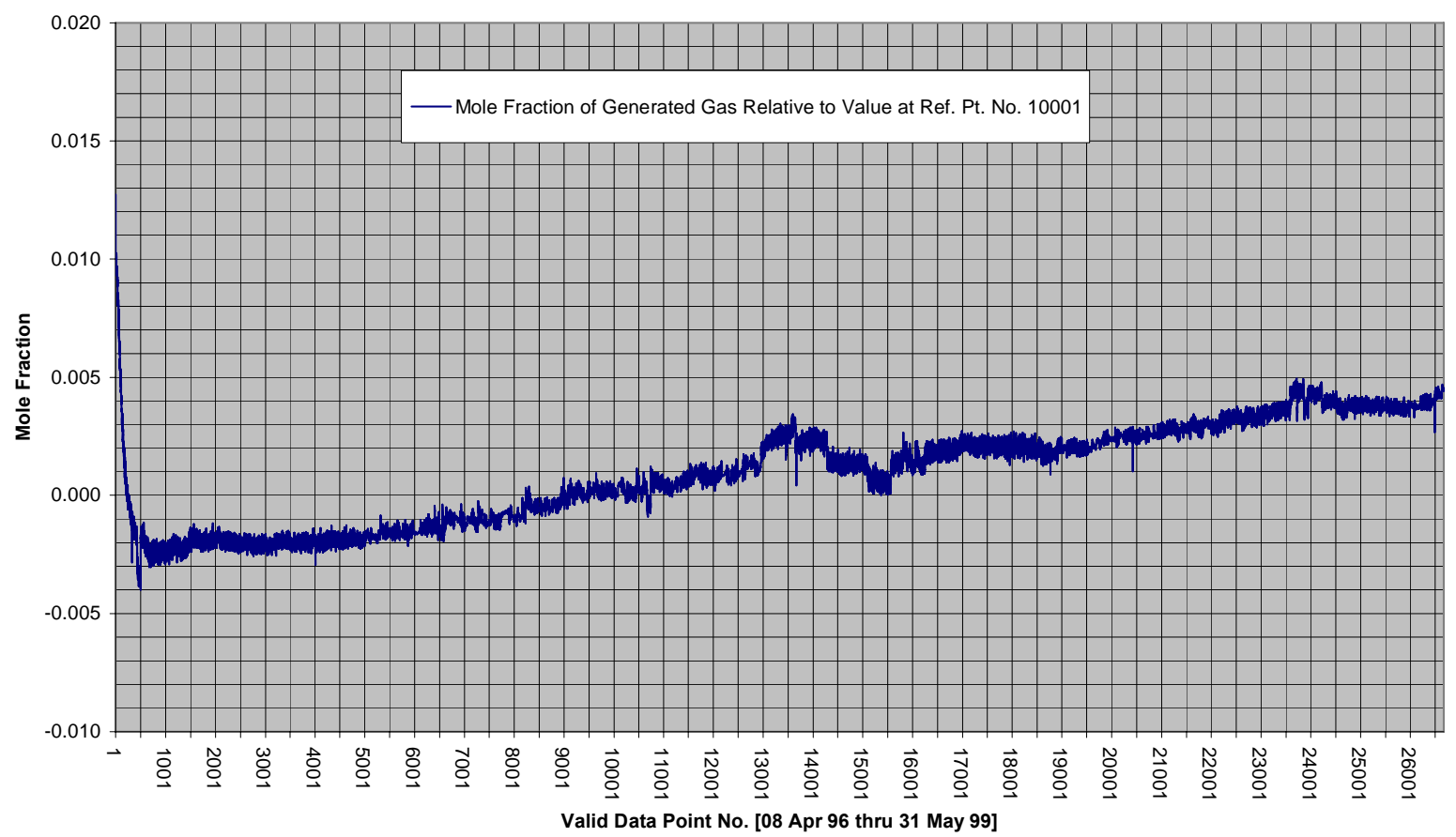

Fig. 32C. Gas Generation, TC-167 


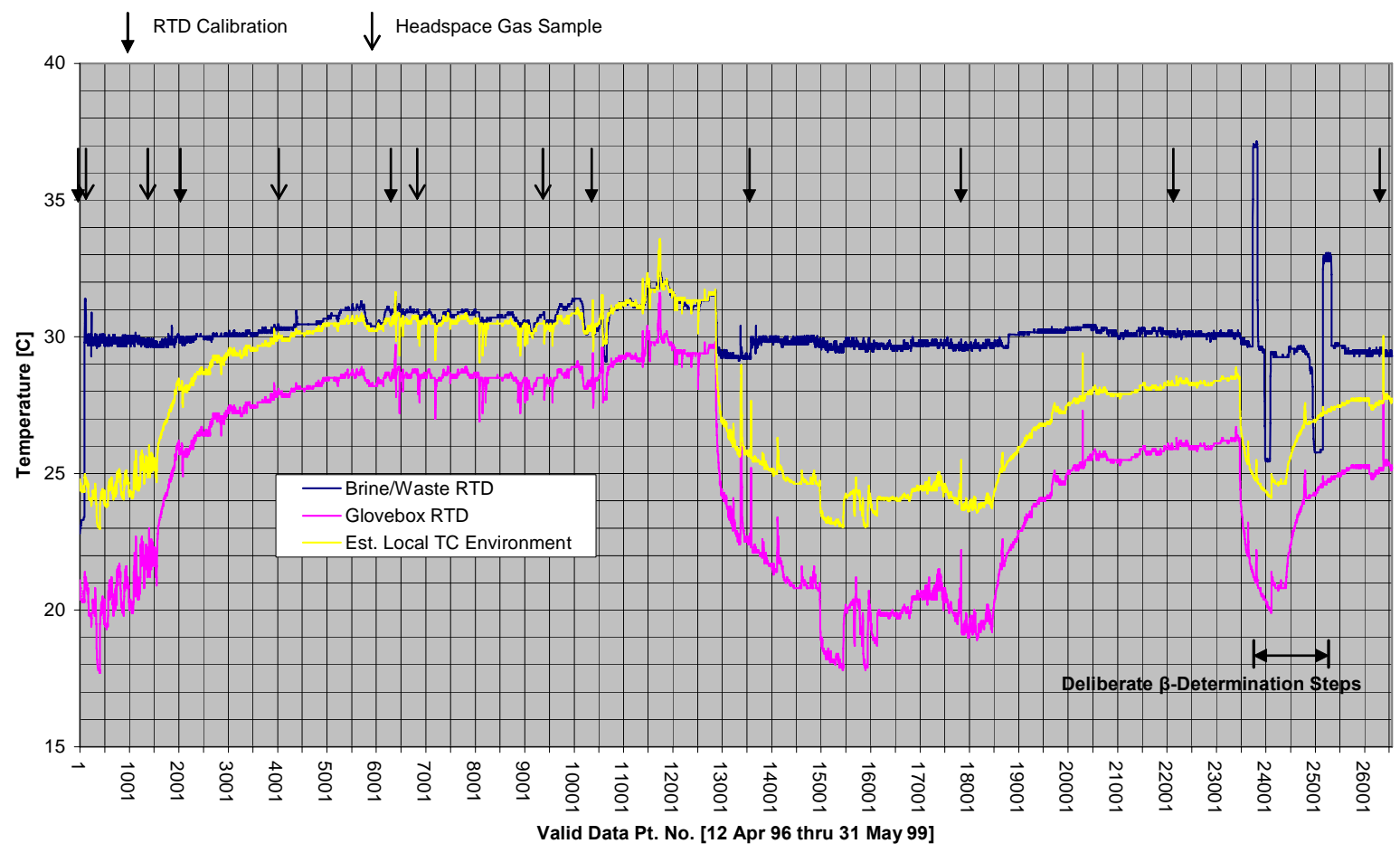

Fig. 33A. Temperatures, TC-168

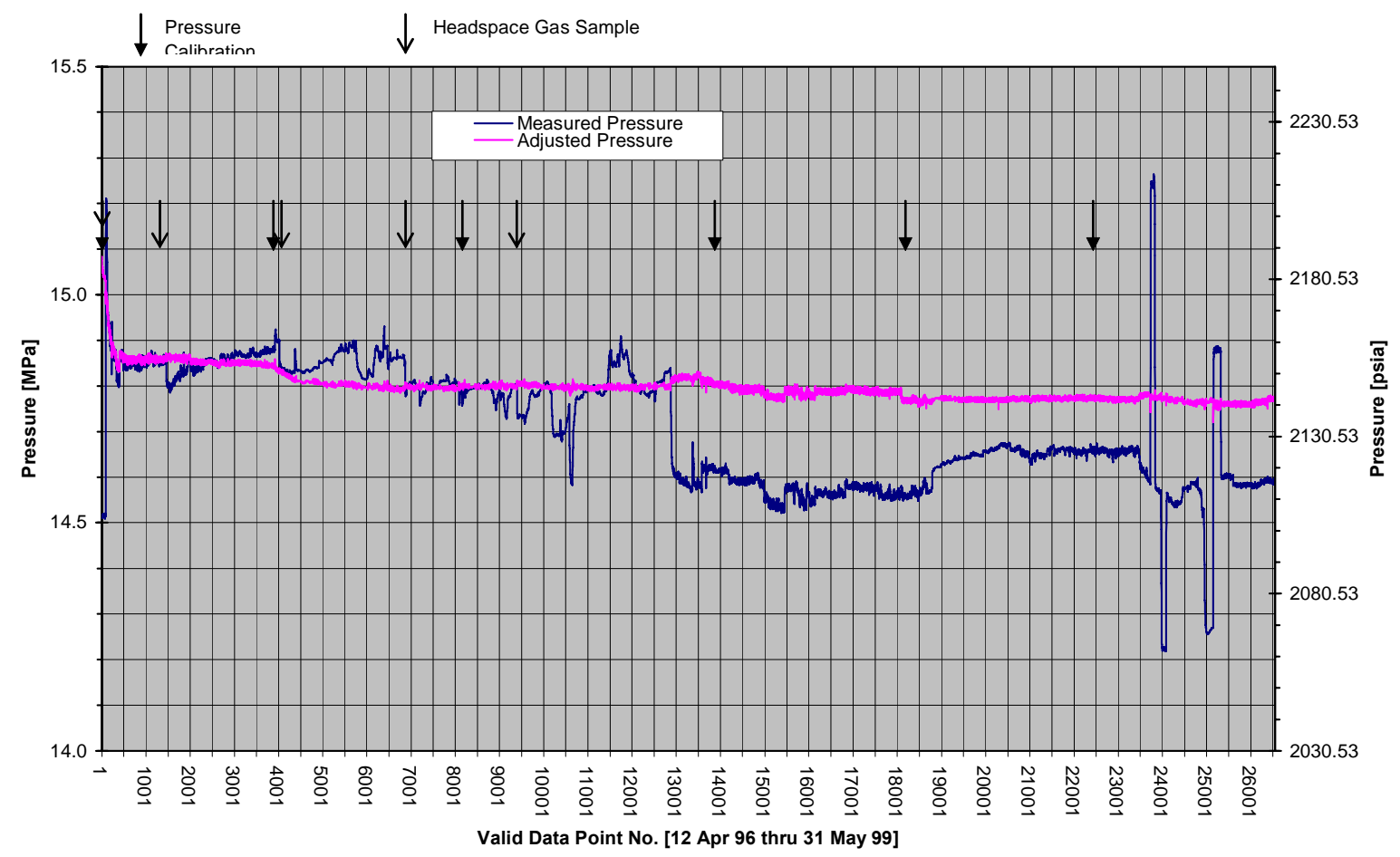

Fig. 33B. Pressures, TC-168 (Ref. Point No. 10001) 


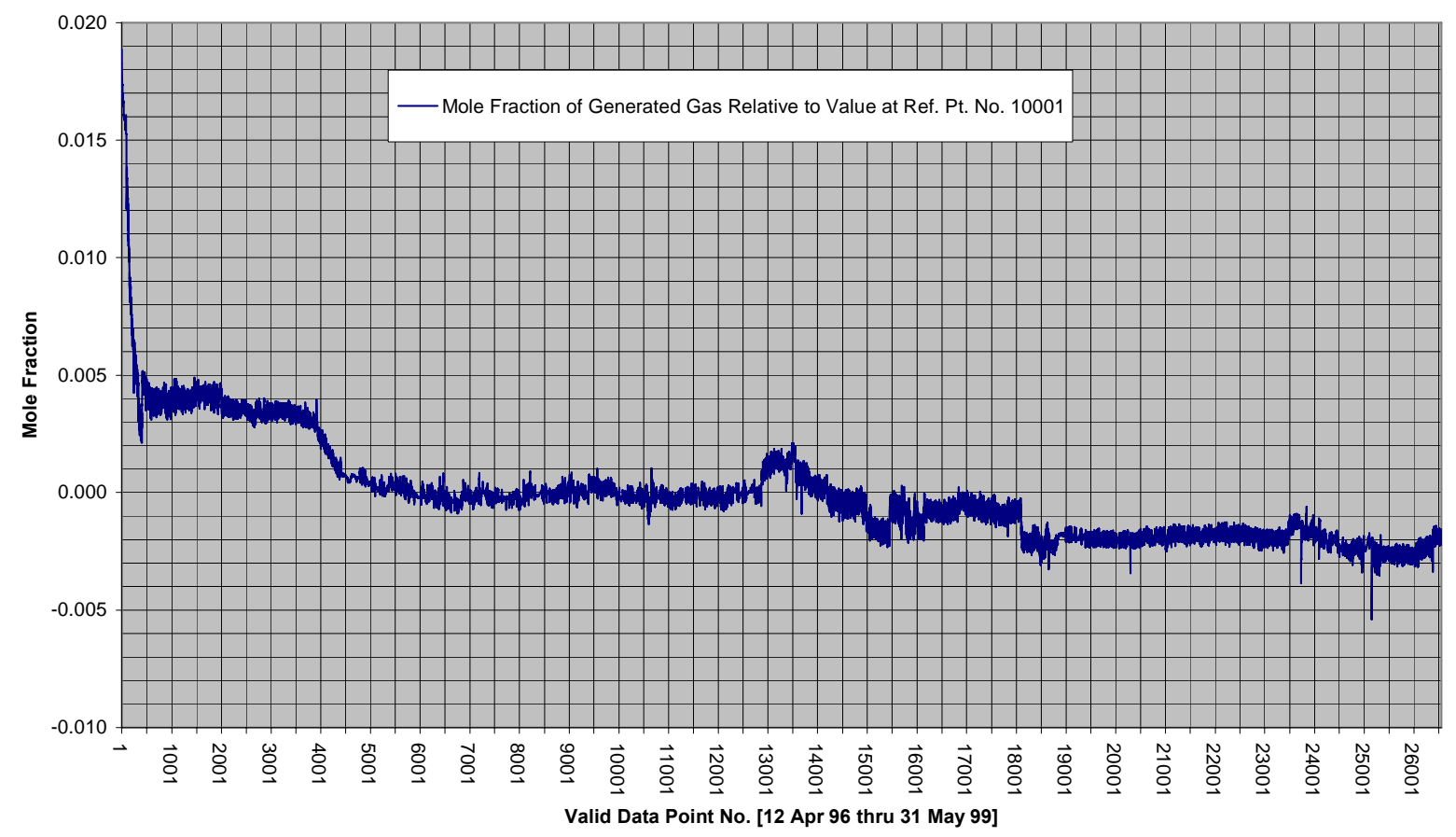

Fig. 33C. Gas Generation, TC-168

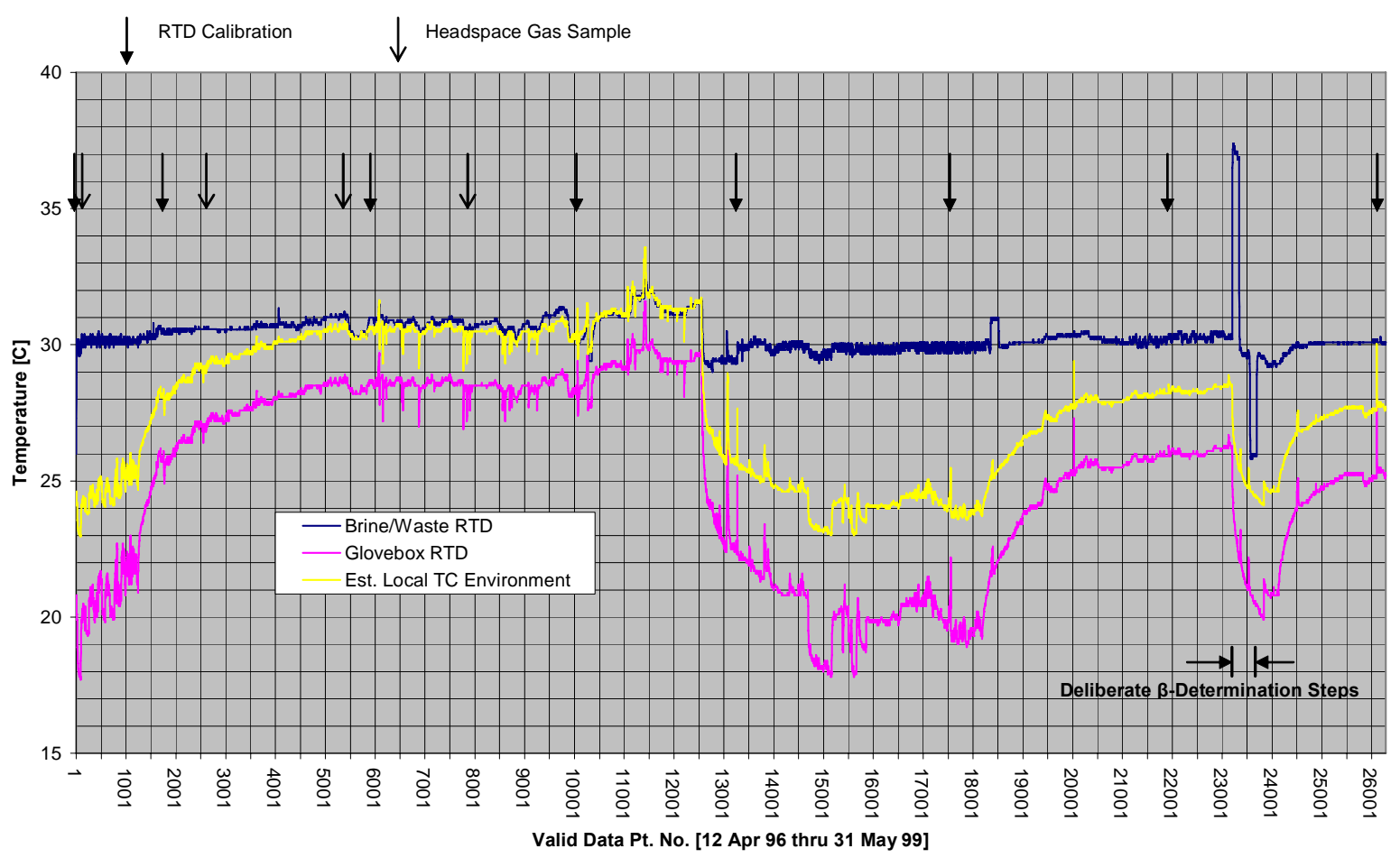

Fig. 34A. Temperatures, TC-169 


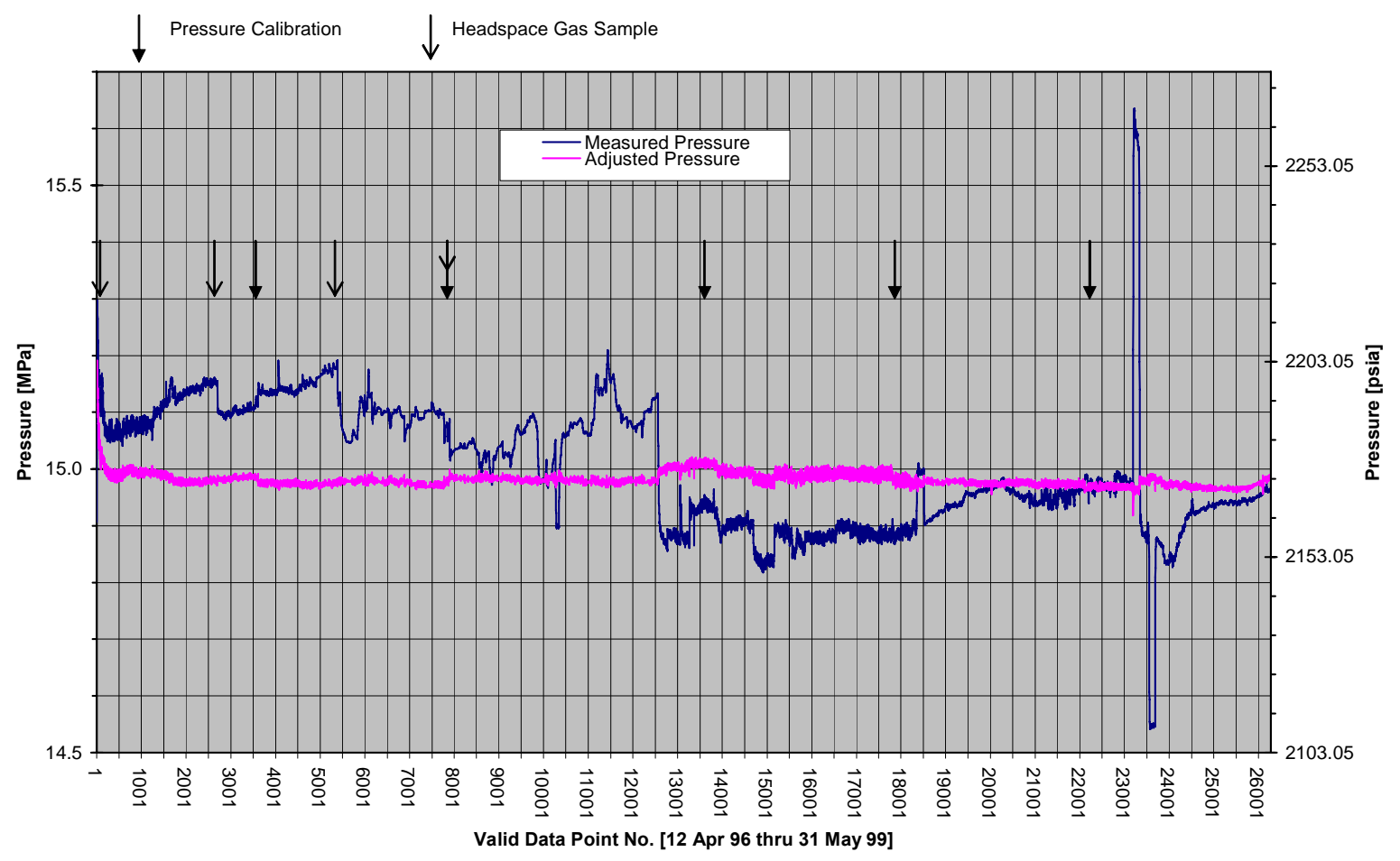

Fig. 34B. Pressures, TC-169 (Ref. Point No. 10001)

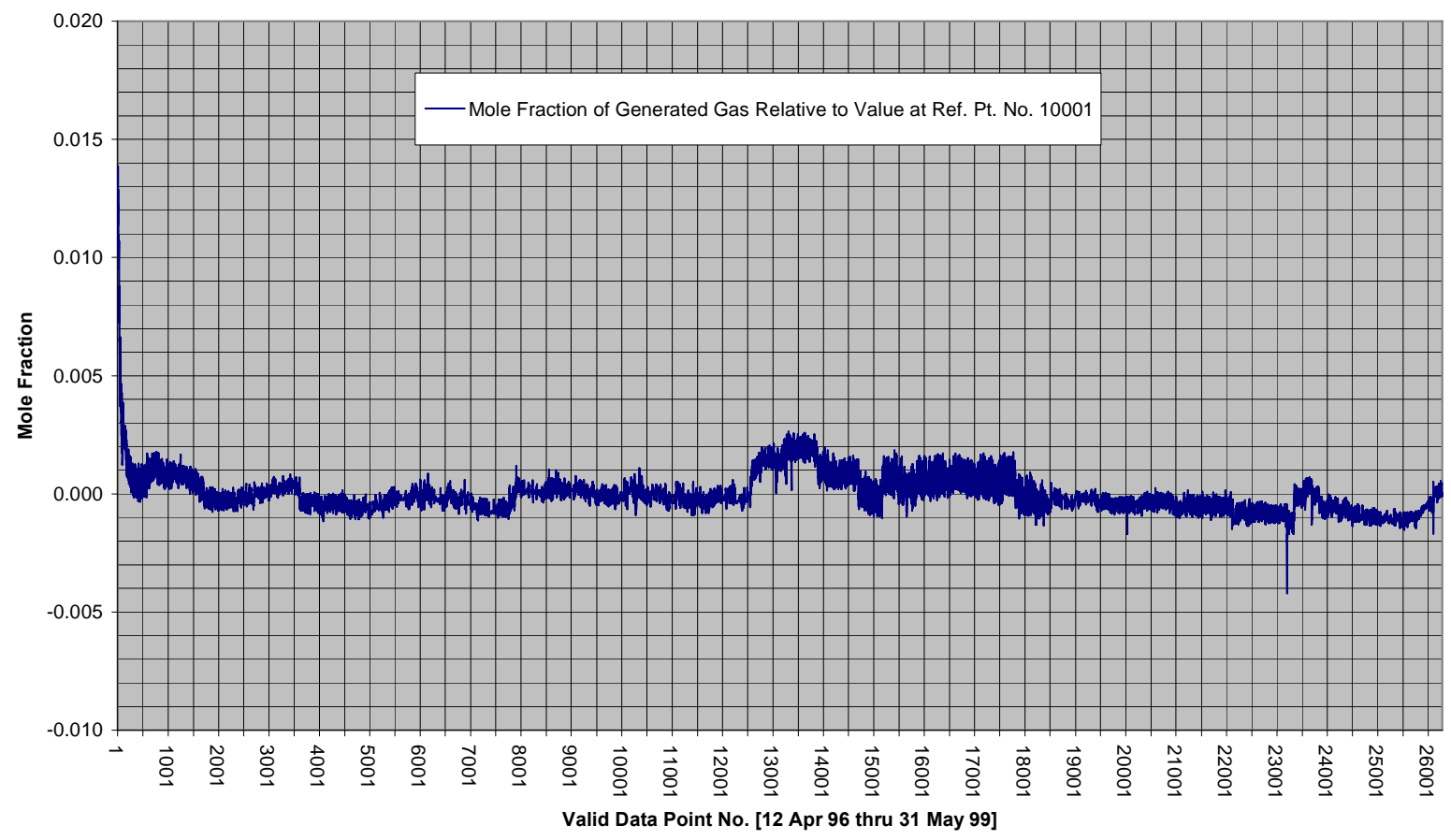

Fig. 34C. Gas Generation, TC-169 


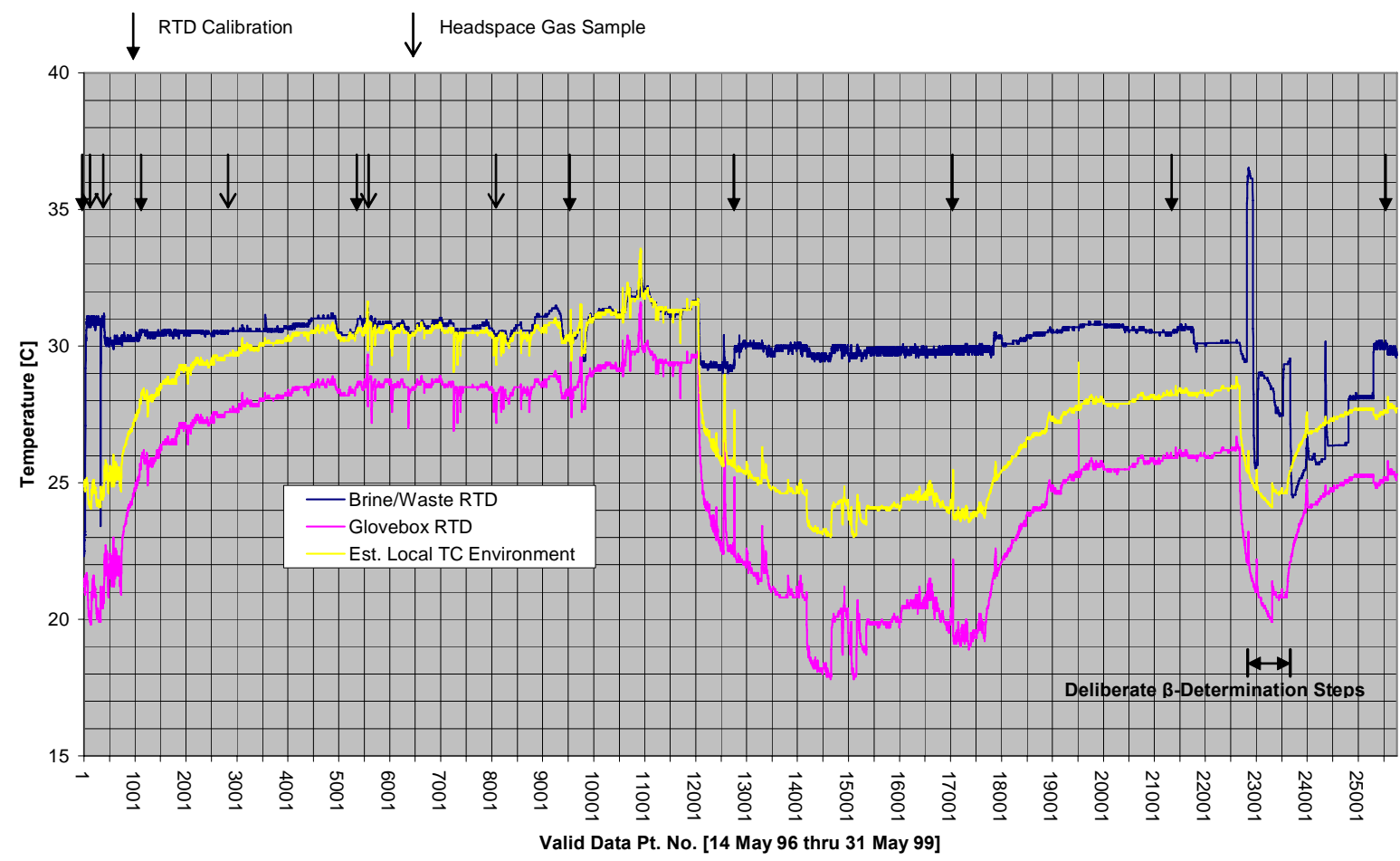

Fig. 35A. Temperatures, TC-170

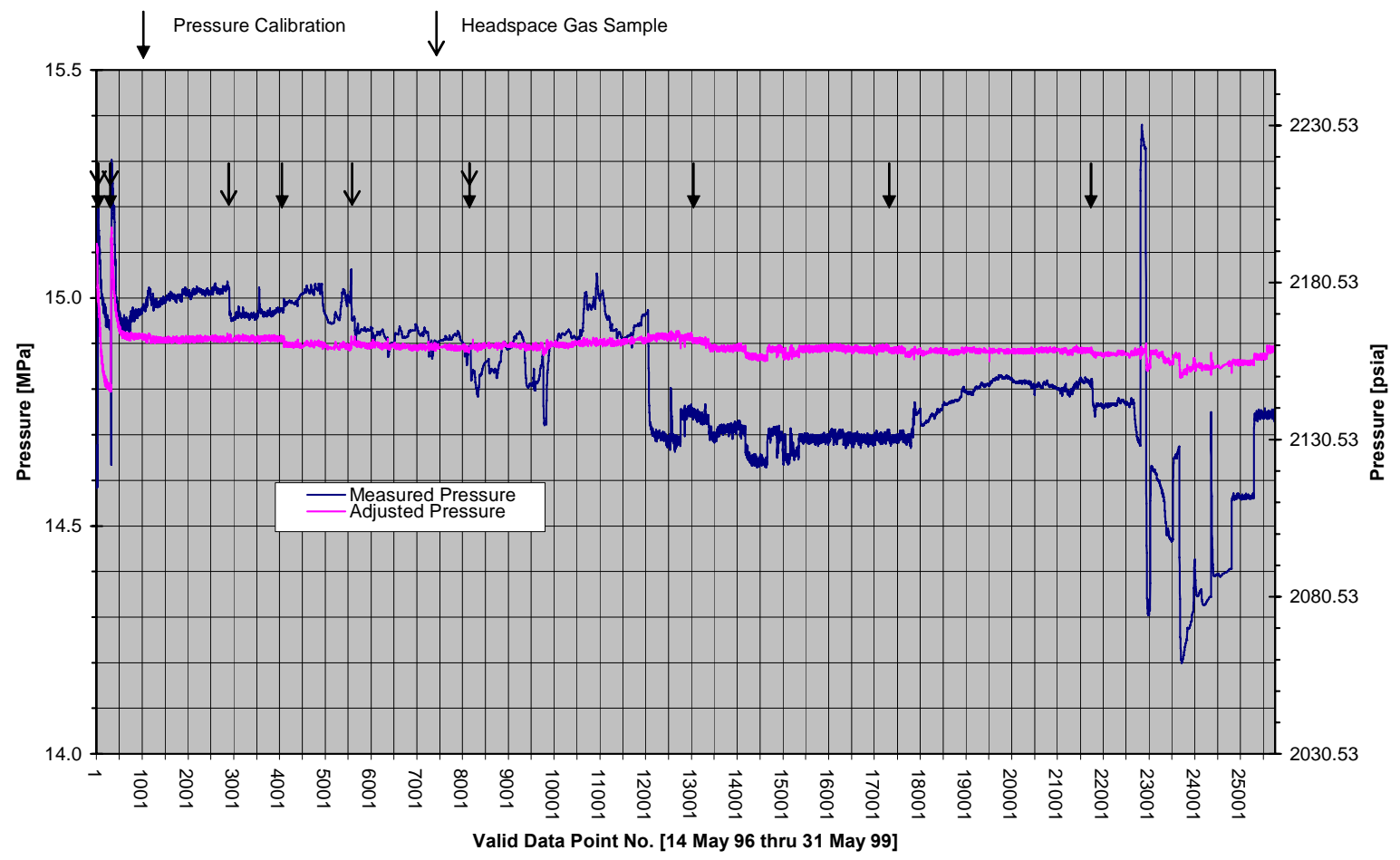

Fig. 35B. Pressures, TC-170 (Ref. Point No. 10001) 


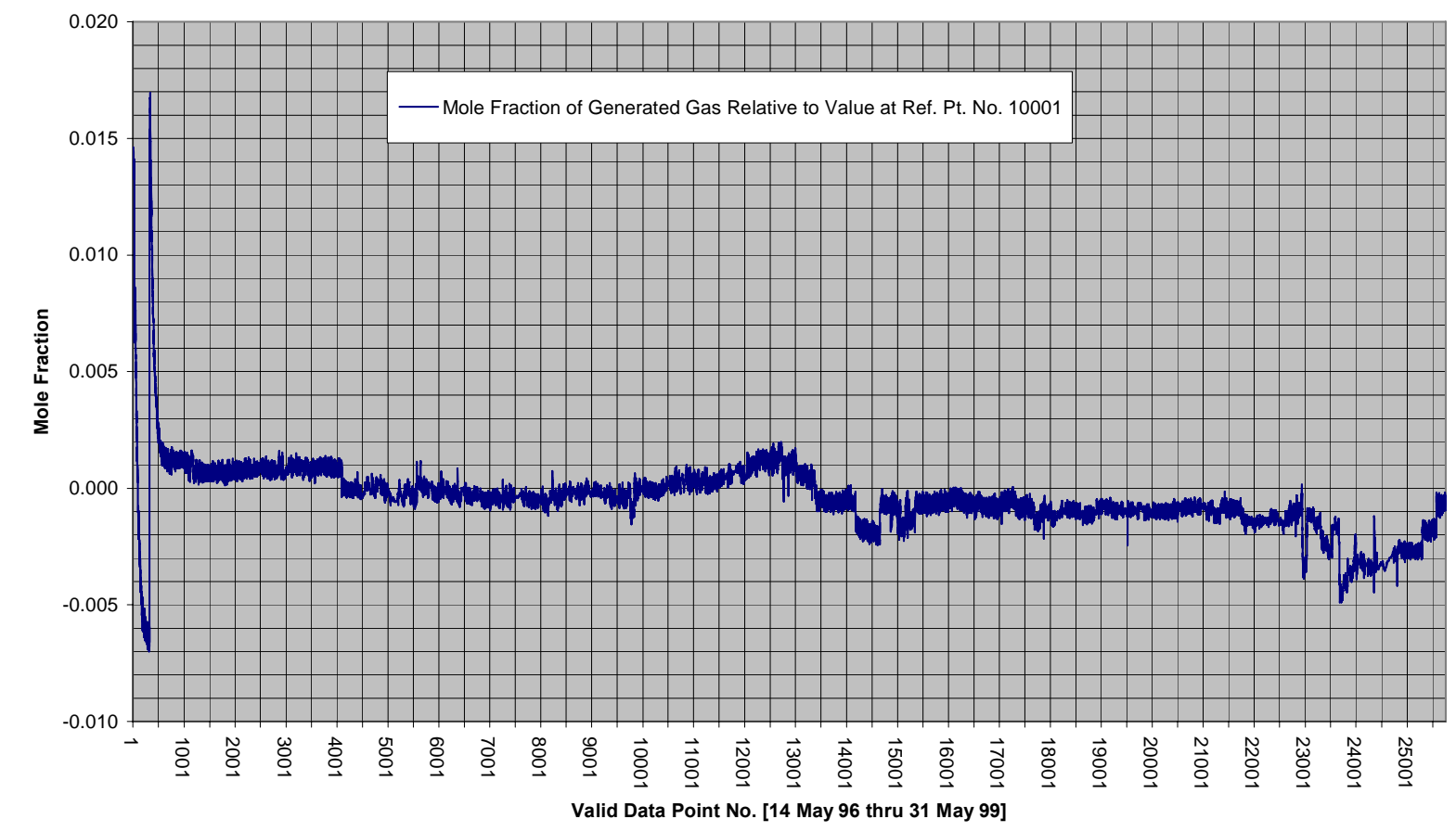

Fig. 35C. Gas Generation, TC-170

\subsection{Discussion}

\subsubsection{Adjusted Pressure Results}

The adjusted-pressure results were generally found to be satisfactory. As was pointed out before, there are numerous uncertainties, parameters, properties, and other aspects that can effect the accuracy of the calculations. These are discussed in Section 13.0. The most suspect adjusted pressures for all of the test containers occurred during the period immediately following the first activation of the ZPPR air-conditioning system on October 22, 1997 (Point 2 on Fig. 8) to alleviate the elevated, summertime temperature in the ZPPR Cell. This rather sudden reduction in the ZPPR-Cell temperature also caused a near-simultaneous drop in the GGE glovebox temperature of about $10^{\circ} \mathrm{C}$.

A possible explanation for the less satisfactory behavior of the adjusted pressures during this period is that the sudden cooling of the glovebox probably caused all of the test-container heaters to be simultaneously energized. This would have caused the "local excess temperature" to deviate drastically from that mapped later, when temperatures had largely stabilized at earlier conditions. This would imply that the algorithm, Eqn. (60), used in the calculation to compute the local excess temperatures during this period may have been less appropriate during this period.

The adjusted pressure calculated for Test Container No. 161 is obviously the least satisfactory of all of the test containers. For this test container there is a notable lack of continuity overall in the adjusted pressure slope. Over the intervals between the discontinuities, the adjusted pressures behave quite well. The discontinuities all occurred precisely at the points where the 
test-container RTD instrument calibrations were performed, as marked in Fig. 26B. This observation prompted a closer scrutiny of all of the calibration data, for which a few ambiguities had been noted earlier in the operations logs.

A purely empirical process was explored for Test Container No. 161 to determine if RTDcalibration corrections, alone, could be responsible for the bizarre behavior of the adjusted pressure. Per the discussion in Section 10.2, it is relatively easy to foresee how instrumentcalibrations factors (both adjustments and residual errors) will affect the adjusted pressure. Figure 36 shows the temperatures for Test Container No. 161 using the combination of RTD calibration factors that would yield the best behaved adjusted pressure. These temperatures are nearly identical to those shown in Fig. 26A. The pressure is shown in Fig. 37. It is clear from Fig. 37 that the discontinuities at the RTD-calibration points have been largely eliminated, lending support to the concern that, although unexplained, errors were introduced in the calibration records for this test container. Table 7 provides a comparison of the recorded calibration factors versus those empirically determined to best eliminate the bothersome characteristics in the adjusted-pressure results for TC-161. However, no definitive resolution could be reached as to why such records for this test container would have been more affected than calibration records for the other test containers.

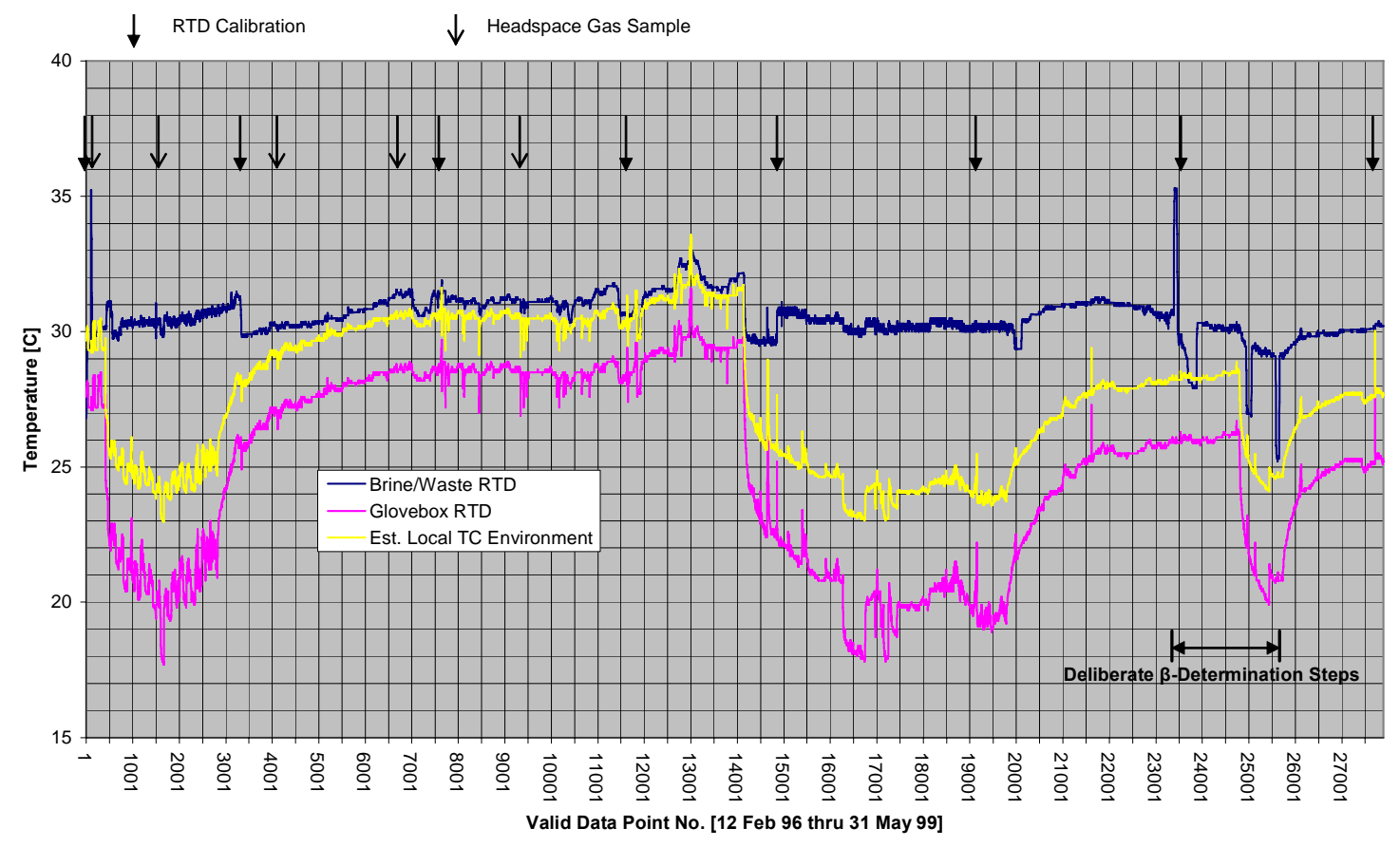

Fig. 36. Temperatures, TC-161 - Empirical RTD-Calibration Corrections 


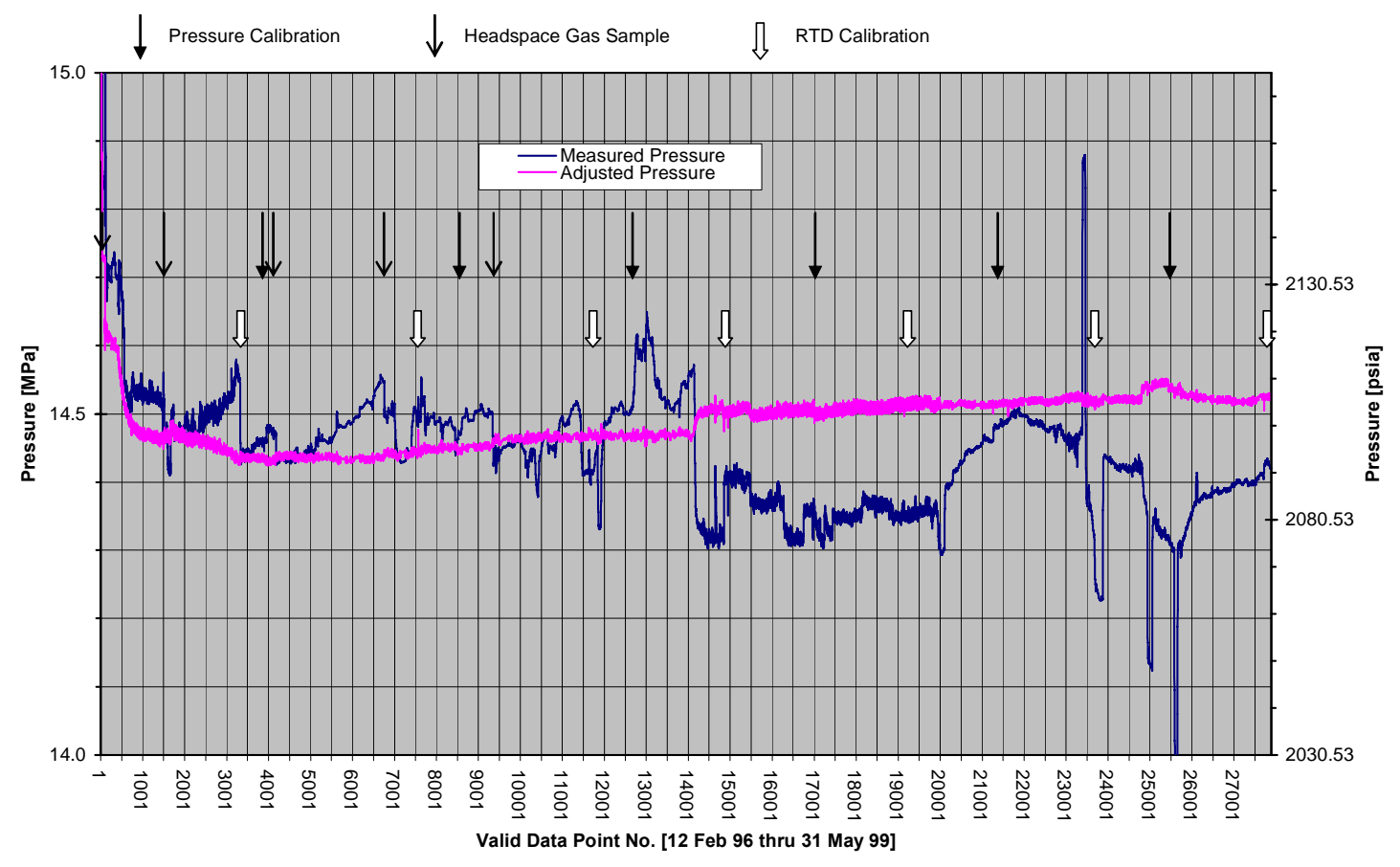

Fig. 37. Pressures, TC-161 (Ref. Point No. 10001) - Empirical RTD-Calibration Corrections

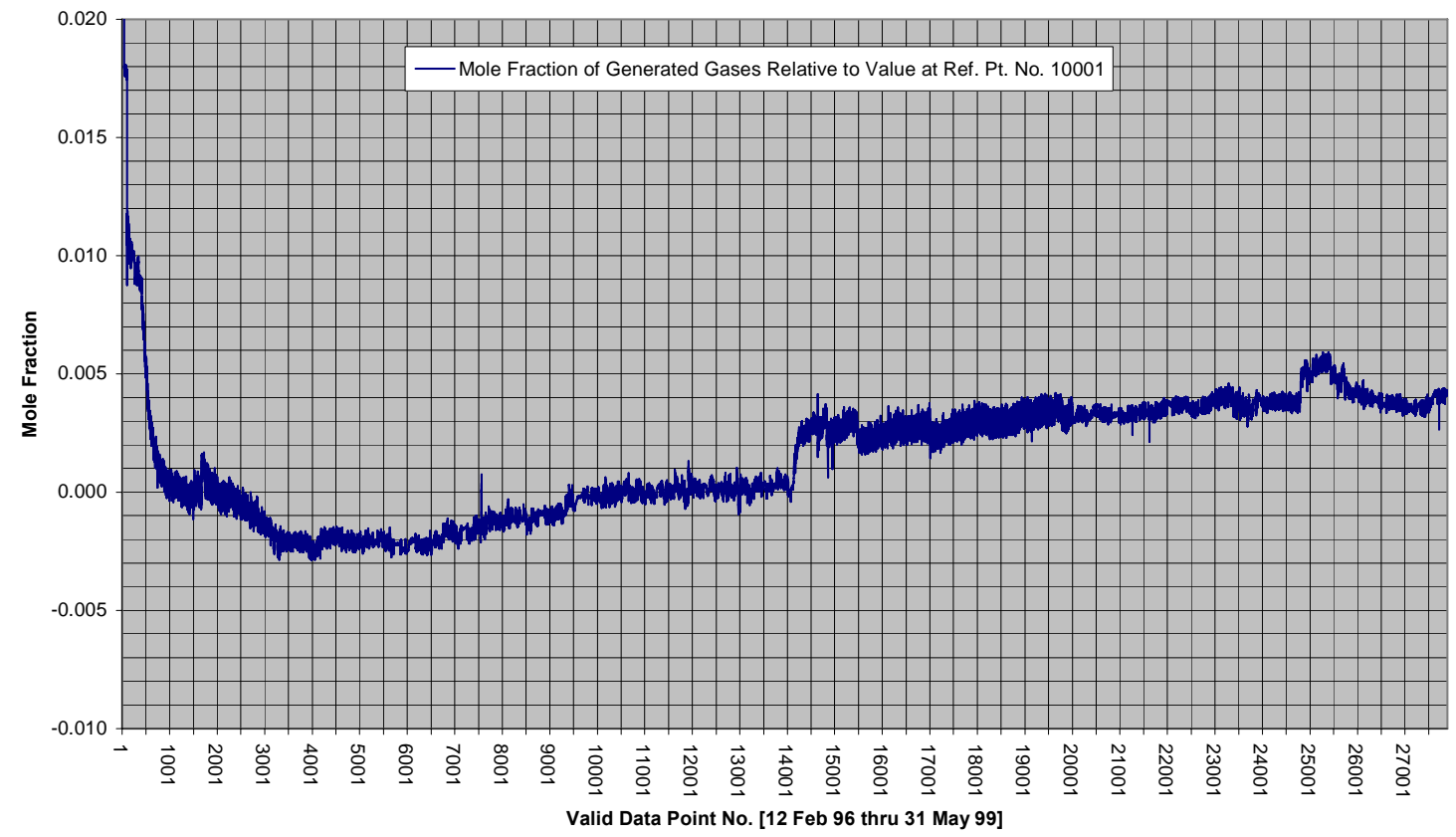

Fig. 38. Gas Generation in TC-161 - Empirical RTD Calibration Corrections 
Table 7. Comparison of RTD Calibration Corrections for TC-161:

Recorded (TC-161) versus Empirical (TC-161e) Corrections

\begin{tabular}{|c|c|c|c|c|c|}
\hline Date & $\begin{array}{c}\text { Data Pt. } \\
\text { No. }\end{array}$ & $\begin{array}{c}\text { Recorded } \\
\text { Adjustment } \\
{[\mathrm{C}]}\end{array}$ & $\begin{array}{c}\text { Recorded } \\
\text { Residual } \\
{[\mathrm{C}]}\end{array}$ & $\begin{array}{c}\text { Empirical } \\
\text { Adjustment } \\
{[\mathrm{C}]}\end{array}$ & $\begin{array}{c}\text { Empirical } \\
\text { Residual } \\
{[\mathrm{C}]}\end{array}$ \\
\hline \hline $02-12-96$ & 1 & 0. & 0. & 0. & 0. \\
\hline $07-15-96$ & 3323 & 0.5 & 0. & 0.8 & 0. \\
\hline $01-13-97$ & 7558 & 0. & 0. & 0.3 & -0.1 \\
\hline $07-09-97$ & 11626 & 0. & 0.1 & -0.35 & 0.45 \\
\hline $11-21-97$ & 14848 & 0.6 & 0.1 & 0.6 & 0. \\
\hline $05-21-98$ & 19142 & -0.9 & 0. & -0.7 & 0.7 \\
\hline $11-21-98$ & 23524 & 0.4 & 0. & 0.6 & -0.1 \\
\hline $05-25-99$ & 27711 & 0. & -0.1 & 0.1 & 0. \\
\hline
\end{tabular}

\subsubsection{Examination for Evidence of Gas Generation}

Figures 24 through Fig. 38, suffix $C$, show the mol fractions of generated gases for Test Containers No. 151 and 160 through 170, respectively, relative to the mol fraction at the indicated reference conditions. Similar gas-generation results for the two test blanks, Test Containers No. 152 and 153, were presented in Section 9.2, Fig. 22 and Fig. 23.

Examination of these figures quickly identifies the test containers in which significant gases are being generated. These are Test Containers No. 151, 160, 161, 166, and 167. Some lesser rates of gas generation might also be perceived in Test Containers No. 162 and 163. There was either no or negligible perceptible gas generation over the 3.25 year test period through May 1999 for the other test containers. Rates of gas generation, inferred per Eqn. (35) by visual examination of the figures, are given in Table 8. The gas-generation results for Test Container No. 161 (Fig. 38) were based on the adjusted pressure recomputed using the empirical RTD-calibration corrections discussed in the previous section and shown in Table 7.

Test Container No. 166 shows a considerably higher gas-generation rate than any of the others. Gas generation seems to have been modest for the initial 9.5 months, but then began at a rather high rate. Omitting the initial period, i.e., prior to about Data Point No.6001, gases are apparently being generated in Test Container No. 166 substantially faster than in the other gasgenerating test containers during the period covered in this report.

Since the objective of this report is only to document the analytical process by which the pressure data was resolved and recovered, further analysis of the gas-generation results is deferred to other GGE program reports. 
Table 8. Graphically Inferred Gas-Generation Rates

\begin{tabular}{|c|c|c|c|c|c|c|c|c|c|}
\hline $\begin{array}{c}\text { Test } \\
\text { Container } \\
\text { No. }\end{array}$ & $\begin{array}{l}\text { Point-b } \\
\text { Point No. }\end{array}$ & $\begin{array}{l}\text { Point-b } \\
\text { Date }\end{array}$ & $\begin{array}{l}\text { Relative } \\
\text { Mol } \\
\text { Fraction } \\
\text { DX }_{\text {gen-b }}\end{array}$ & $\begin{array}{c}\text { Point-a } \\
\text { Point No. }\end{array}$ & $\begin{array}{c}\text { Point-a } \\
\text { Date }\end{array}$ & $\begin{array}{l}\text { Relative } \\
\quad \text { Mol } \\
\text { Fraction } \\
\text { DX }_{\text {gen-a }}\end{array}$ & $\begin{array}{c}\text { Elapsed Time } \\
\text { Interval, t } \\
\text { [days: years] }\end{array}$ & $\mathrm{dX}_{\text {gen }}$ & $\begin{array}{l}\mathrm{dX}_{\mathrm{gen}} / \mathrm{dt} \\
{[\mathrm{ppm} / \mathrm{yr}]}\end{array}$ \\
\hline 151 & 12501 & $11-17-97$ & 0.0015 & 4501 & $12-08-96$ & -0.0015 & 344: 0.942 & 0.0030 & 3183 \\
\hline 152 & 11501 & 10-19-97 & 0.0000 & 5501 & 02-03-97 & 0.0000 & 258: 0.707 & 0. & 0 \\
\hline 153 & 23501 & 03-19-99 & -0.0018 & 14501 & $02-25-98$ & -0.0018 & $387: 1.060$ & 0. & 0 \\
\hline 160 & 12501 & 09-26-97 & 0.0015 & 4501 & $10-17-96$ & -0.0033 & 344: 0.942 & 0.0048 & 5096 \\
\hline $161 \mathrm{e}$ & 24501 & 01-08-99 & 0.0040 & 10501 & 05-23-97 & 0.0000 & 595: 1.630 & 0.0040 & 2454 \\
\hline 162 & 23501 & $12-07-98$ & 0.0007 & 4001 & $08-27-96$ & -0.0008 & 832: 2.279 & 0.0015 & 658 \\
\hline 163 & 23001 & $11-29-98$ & 0.0010 & 5001 & $10-20-96$ & -0.0005 & 770: 2.110 & 0.0015 & 711 \\
\hline 164 & 22501 & 11-19-98 & 0.0002 & 6501 & 01-02-97 & -0.0005 & 686: 1.879 & 0.0007 & 373 \\
\hline 165 & 23001 & $12-07-98$ & 0.0000 & 5001 & $10-28-96$ & -0.0005 & 770: 2.110 & 0.0005 & 237 \\
\hline 166 & 24501 & 03-04-99 & 0.0110 & 16001 & $02-22-98$ & 0.0030 & 375: 1.027 & 0.0080 & 7790 \\
\hline 167 & 23501 & 01-17-99 & 0.0035 & 4001 & $10-03-96$ & -0.0020 & 836: 2.290 & 0.0055 & 2402 \\
\hline 168 & 12501 & $10-07-97$ & 0.0000 & 6001 & $10-06-96$ & 0.0000 & 366: 1.003 & 0. & 0 \\
\hline 169 & 22001 & 11-26-98 & -0.0003 & 4001 & $10-20-96$ & -0.0003 & 767: 2.101 & 0. & 0 \\
\hline 170 & 21501 & $11-27-98$ & -0.0008 & 15501 & 03-17-98 & -0.0008 & 255: 0.699 & 0. & 0 \\
\hline
\end{tabular}




\subsection{Applicability to Other Investigations and Experiment-Design Implications}

The GGE program consistently maintained the test containers well within the $+/-5^{\circ} \mathrm{C}$ experiment specifications for temperature. Nevertheless, the pressure data collected in the ANL GGE program was found to be unusable without the compensation methodology described in this report, and it was clearly established that even the very small temperature variations that did occur were the primary contributor to the pressure fluctuations observed. The question arises as to whether other gas-generation experiments have experienced similar pressure behavior, and, if not, why not? The answer to this question is discussed in this section, and provides useful guidance to the design of future experiments.

The GGE program used very prototypic conditions. The pressure was selected to match the very high lithostatic pressure expected eventually at WIPP, and waste compositions, packing, and radioactivity levels were typical of those TRU wastes destined for WIPP. These conditions are key to the following discussion.

Equation (2) shows how the pressure, temperature, mass, and volume at a given state are related. Assume that in the vicinity of a given set of operating conditions the compressibility, $z$, and the headspace-gas mass, $M$, are constant. Then the pressure variation induced by variations in temperature or headspace-gas volume can be found by differentiating Eqn. (2), giving

$$
d P=z M R \frac{V_{H S} d T-T d V_{H S}}{V_{H S}^{2}}
$$

This can be rearranged to

$$
d P=\frac{z M R T}{V_{H S}}\left(\frac{d T}{T}-\frac{d V_{H S}}{V_{H S}}\right)=P\left(\frac{d T}{T}-\frac{d V_{H S}}{V_{H S}}\right)
$$

Upon expressing these differentials as small but finite perturbations about the original operating point, denoted here by a zero subscript (The zero subscript here refers simply to a given operating point, not the overall reference point for the compensation calculation, as used elsewhere in this report.)

$$
\delta P=P_{o}\left(\frac{\delta T}{T_{o}}-\frac{\delta V_{H S}}{V_{H S_{o}}}\right)
$$

For the sake of algebraic simplicity, consider the case where there are no changes in the volume of the test container (this effect is usually very small compared to the volume change in the brine/ waste, as was demonstrated in the example in Section 9.1). Volume variation in the brine/waste has been considered to occur only from thermal expansion/contraction effects (see discussion in Section 6.1.2). Again, for algebraic simplicity, express the volume change in the brine/waste, $d V_{l}$, as in Eqn. (42), rather than as in Eqn. (43). With these simplifications, 


$$
\delta V=-\delta V_{l}=\beta V_{l_{O}} \delta T
$$

Substituting Eqn. (84) into Eqn. (83) and rearranging shows that the sensitivity of the pressure to variations in temperature will be

$$
\delta P=P_{0}\left(\frac{1}{T_{o}}+\frac{\beta}{V_{H S_{o}} / V_{l_{o}}}\right) \delta T
$$

\subsection{Pressure Sensitivity to Operating Pressure}

Consider the situation where two experiments have identical ratios of headspace and liquid, i.e., the term $V_{H S-0} / V_{l-0}$ is the same for both experiments (regardless of the test-container volume). With the same brine/waste (i.e., equal $\beta$ ) and operating at the same temperature, $T_{0}$, Eqn. (85) shows that the pressure perturbation is proportional to the operating pressure. An identical temperature perturbation, $d T$, in both experiments produces pressure perturbations that are proportionately more pronounced in the higher operating-pressure test container.

\subsection{Pressure Sensitivity to Headspace/Waste Volume Ratio}

Most investigations into gas-generation behavior monitor the experiment pressure to surmise the extent of gross gas generation. An objective of the GGE program was to identify the specific gases generated. Pressure measurements were intended only to provide backup information. Furthermore, the GGE program used actual TRU wastes immersed in brine with prototypic waste packing. Other investigations frequently overload the experiments with metals to artificially increase the quantity of gases generated over a specified time interval for a given brine/waste volume. This non-prototypicality produces measurable generated gas quantities rapidly, with correspondingly rapid pressure increases, even in relatively large headspace-gas volumes. On the other hand, the prototypic GGE program required relatively small headspace volumes to develop measurable generated-gas concentrations in the headspace.

Consider, again, Eqn. (85), but now from the viewpoint of two experiments with the same brine/ waste, operating at the same temperature and pressure, but which have different ratios of headspace volume to brine/waste volume, $V_{H S-0} / V_{l-0}$. Once more, the actual volume of the test container is unimportant. Since all of the terms in Eqn. (85) are positive quantities, the experiment having the smaller headspace-to-brine/waste volume ratio will experience the greater pressure perturbation for a given temperature perturbation.

\subsection{Comparison of the GGE to Other Gas-Generation Experiments}

GGE test containers typically operated at approximately $14.824 \mathrm{MPa}$ (2150 psia), about 146 standard atmospheres, and $30^{\circ} \mathrm{C}$. The test containers were loaded to headspace-to-brine/ waste volume ratios on the order of 0.1 . The volumetric coefficient of thermal expansion in the

brine/waste was found to be typically on the order of $0.38 \mathrm{E}-3 \mathrm{~K}^{-1}$. Evaluating Eqn. (85) with these parameters shows that the GGE should anticipate a pressure sensitivity of 


$$
\frac{\delta P}{\delta T}=14.824 M P a\left[\frac{1}{303.15 K}+\frac{0.38 E-3 K^{-1}}{0.1}\right]=105.49 \mathrm{kPa} / \mathrm{C}(15.3 \mathrm{psi} / \mathrm{C})
$$

The example in Section 9.1 showed that the sensitivity of the GGE experiments to temperature variation was, indeed, observed to have about this value.

Sections 12.1 and 12.2 showed that experiments conducted at pressures lower than those in the GGE program and/or those having larger ratios of headspace volume to brine/waste volume would experience smaller pressure perturbations. As an interesting comparison, Ref. 9 is an excellent report on the results of an investigation into gas-generation mechanisms conducted by Pacific Northwest National Laboratory on behalf of Sandia National Laboratory. Those experiments used the same Brine- $A$ formulation as in GGE. The experiments were typically run at about $10 \mathrm{~atm}$ pressure, and with the same temperature specification of $30+/-5^{\circ} \mathrm{C}$ as in the GGE program. Headspace-to-brine/waste volume ratios, however, were approximately 0.37 (ibid., Section 6.1.1). Under these conditions, and assuming comparable brine/waste thermalexpansion coefficients, Eqn. (85) shows that the pressure sensitivity in those experiments should be

$$
\frac{\delta P}{\delta T}=1.0135 \mathrm{MPa}\left[\frac{1}{303.15 \mathrm{~K}}+\frac{0.38 E-3 \mathrm{~K}^{-1}}{0.37}\right]=4.413 \mathrm{kPa} / \mathrm{C}(0.64 \mathrm{psi} / \mathrm{C})
$$

This is far less than the $105.49 \mathrm{kPa} / \mathrm{C}(15.3 \mathrm{psi} / \mathrm{C})$ found in the GGE, and accounts for the very smooth pressure measurements reported in Ref. 9.

\subsection{Limitations of the Analyses}

The methodology presented here is far from perfect. There are numerous limitations imposed on the reconstruction of the pressure data by shortcomings in the method of analysis and, in a few instances, by inaccuracies in the data. The list below identifies numerous potential pitfalls in both areas.

1. The experiment and the temperature and pressure behavior are transient, whereas the compensation methodology treats each data point as a steady-state condition. The entire pressure response is presumed to be well represented by a succession of steady state variations, not just point to adjacent point, but also between a point and the reference point.

2. The thermal expansion/contraction coefficients were based on the temperature-step data produced deliberately and with some degree of uncertainty. These were also generated at one point in time, after the experiments had been running for nearly three years. The value so found was then applied to the entire data set over the multiyear time frame. Any changes that may have occurred in this parameter over time would not have been known.

3. There were calibration corrections frequently made to both the RTD temperature sensors as well as to the pressure transducers. Although corrections were made, the 
rate of degradation was not known. Degradation of these instruments was assumed to have occurred through a gradual and uniform process between consecutive calibration points.

4. The local temperature within the glovebox was estimated from some simple experiments under limited conditions. It is known that the local temperature is influenced somewhat by operation of the argon gas circulators, the number and location of the test-container heaters that are on at any one time, etc., but these secondary effects were not quantitatively explored.

5. Any leakage of headspace gas from the test containers would affect the true pressure data. The perfect containment of high-pressure gas at about $14.824 \mathrm{MPa}$ (2150 psig) over periods of several years in a mechanically fastened container without the use of elastomer seals and subjected to thermal cycling is challenging.

6. At any one time there were perturbations to the system from calibrations, routine maintenance of equipment, equipment repairs and change out, equipment malfunctions (e.g., the MO-120 gas-purification unit), power outages, etc., all of which introduce some degree of upset to the system.

7. The test-container heaters were intended to control the test-container (RTD) temperature, but the feedback loop was sensing an auxiliary thermocouple mounted on the outside of the test container jacket, rather than the test-container RTD.

8. The off-line experimental measurement of $\beta$ for brine was made at atmospheric pressure instead of at the high operating pressure. Published density data for water shows some slight effect of pressure.

9. There are a host of physical properties that are used in the methodology, particularly the thermal model, that may contain some error. For some properties, the brine was approximated as being water (e.g., the solubility treatment used parameters that pertain to gas diffusion in water).

10. The smearing of penetrations and other features in the head region to axisymmetrical geometry compromised the thermal models to some extent.

11. Assumptions had to be made for gap and contact conductances in the thermal models.

12. The role of solubility in determining the headspace-gas mass was estimated from a simplified model of the transport characteristics of gas diffusion in the liquid.

13. The experimental determination of the headspace volume may have introduced some error.

14. The physical dimensions used for transport surface areas were best estimates. 
15. The idealized convection models used in the brine and in the headspace in the thermal models probably introduced some error.

16. The simplified one-dimensional thermal model and the constant-factor approach taken for handling two-dimensional effects in the test-container head region was known to be only a crude approximation.

17. There are angular non-uniformities in the heating (the heater bands covered only about 300 degrees of the test container circumference, whereas the thermal model is based on uniform circumferential conditions. The 60-degree gaps were not uniformly located on all the containers.

18. The dead bands in the heater controller cause lags in the temperature control.

19. Roundoff of temperature and pressure measurements to one decimal place were made by the data-acquisition system.

20. There were occasionally anomalous data recorded in the database.

21. There were some small discrepancies in the instrument-calibration records noted as to whether consistent terminology was used regarding how the instrument read relative to the standard during calibrations, and some incompleteness in the records was noted.

22. Generated gases originate as bubbles that may remain attached to the waste, or trapped and prevented from reaching the headspace. This does not affect the pressure response, but would allow the gases to be dissolved in the brine more readily (this would also make the headspace gas composition as determined from samples non-representative of the true composition).

Considering all of the many limitations listed above, the best criterion for evaluating the worth of the effort is the comparison of the compensated pressures at specific points to the measured data for the blank, control vessels, Test Containers No. 152 and 153. These were developed in Section 9.1 (Fig. 20 and Fig. 21). On that basis, the methodology appears to provide very acceptable results. 


\section{REFERENCES}

1. L. Haar, J. S. Gallagher, and G. S. Kell, "NBS/NRC Steam Tables - Thermodynamic and Transport Properties and Computer Programs for Vapor and Liquid States of Water in SI Units,” Hemisphere Publishing Corporation, Washington, D. C. (1984).

2. G. J. Van Wylen, “Thermodynamics,” John Wiley \& Sons, New York (1963).

3. D. R. Lide, editor, "Handbook of Chemistry and Physics," $74^{\text {th }}$ ed., Chemical Rubber Co., CRC Press, Boca Raton (1993).

4. J. A. Dean, editor, “Lange's Handbook of Chemistry,” $14^{\text {th }}$ ed., McGraw-Hill, Inc, New York (1992).

5. N. Ernest Dorsey, editor, "Properties of Ordinary Water Substance," American Chemical Society, Book Dept., Reinhold Publishing Corp., New York (1940).

6. W. F. Linke, editor, "Solubilities - Inorganic and Metal-Organic Compounds, K-Z; A Compilation of Solubility Data from the Periodic Literature," $4^{\text {th }}$ ed., 2, American Chemical Society, Washington, D.C. (1965).

7. C. L. Yaws, et. al., "Calculating Solubility and Henry's Law Constants for Gases in Water," Chem. Eng. 106(6): (June 1999).

8. P. R. Hart to D. G. Cummings, Argonne National Laboratory-West, personal communication (August 18, 1995).

9. M. R. Telander and R. E. Westerman, "Hydrogen Generation by Metal Corrosion in Simulated Waste Isolation Pilot Plant Environments," Battelle Pacific Northwest Laboratory Report, SAND96-2538 (March 1997).

10. American Society for Metals, “ASM Metals Reference Book,” 3:155, Metals Park, OH (1993).

11. Y.Li and S. Gregory, "Diffusion of Ions in Sea Water and in Deep Sea Sediments," Geochimica et Cosmochimica Acta, 38:703-714 (May 1974).

12. H. S. Carslaw and J. C. Jaeger, "The Conduction of Heat in Solids," $2^{\text {nd }}$ Ed., Oxford Press, London, England (1959).

13. J. M. Coulsen and J. F. Richardson, “Chemical Engineering,” Revised $2^{\text {nd }}$ Ed., 1, Pergamon Press, Oxford England (1965).

14. E. R. G. Eckert and R. M. Drake, Jr., “Analysis of Heat and Mass Transfer,” McGraw-Hill Book Company, New York (1972). 
15. J. Barnes to F. S. Felisione, Bechtel BWXT Idaho Technologies, personal communication (1999).

16. Riley, editor “Chemical Oceanography,” 2nd Ed., 4:338 (1975).

\section{ACKNOWLEDGMENT}

This work was part of an ANL-West effort supported by the DOE Carlsbad Field Office through SNL. Principal Investigator was Andrew Peterson, SNL. The funding support provided by Dave Duncan, former ANL-West GGE Project Manager and the continued encouragement by Carla Dwight, former ANL-West Waste Programs Manager, made the work possible and kept the study moving forward.

The assistance and support of several colleagues at ANL-West is gratefully acknowledged. Conversations with Joe Mitchell and Dan Cummings concerning the initial efforts to resolve this problem were very helpful and appreciated. Bryan Forsmann and Gary Schwartzenberger set up and conscientiously maintained the auxiliary experiment apparatus used for making the measurements of the thermal-expansion coefficients for the brine. Larry Foulkrod was very supportive and generous with his time whenever called upon to make numerous adjustments to the actual GGE experiment equipment needed to support this investigation. 


\section{APPENDIX A}

\section{Gas-Solubility Variation}

The formulation used in the compensation methodology considered the effects of gas solubility in the brine. Solubility parameters were taken from the literature to specify the quantity of gas dissolved in the brine under given temperature and pressure conditions. These were equilibrium values. It is instructive to examine the rate of transport to assess the extent to which equilibrium values are relevant.

\section{A1. Diffusion Model}

Consider first a limiting case where all transport of gas through the brine is by diffusion only. This is an easily obtained solution that, although unrealistic, provides some feel for the problem. The 361-mm-deep body of brine liquid is in contact with headspace gas at some given pressure. The concentration of gas throughout the liquid is initially uniform at the equilibrium value, $C_{0}$, that corresponds to the initial conditions. At time zero conditions change, causing the dissolvedgas concentration to seek a new equilibrium value, $C_{2}$. In an infinitesimally thin layer at the surface, the gas concentration can be assumed to instantaneously achieve the value, $C_{2}$, which could be found from Henry's Law, which states that, at constant temperature, the mole fraction of a dissolved gas is proportional to the partial pressure of that gas above the liquid surface. The physical arrangement and the general behavior of the dissolved-gas concentration is shown in Fig. A1. The diffusion of gas through the brine is described by (Ref. 13)

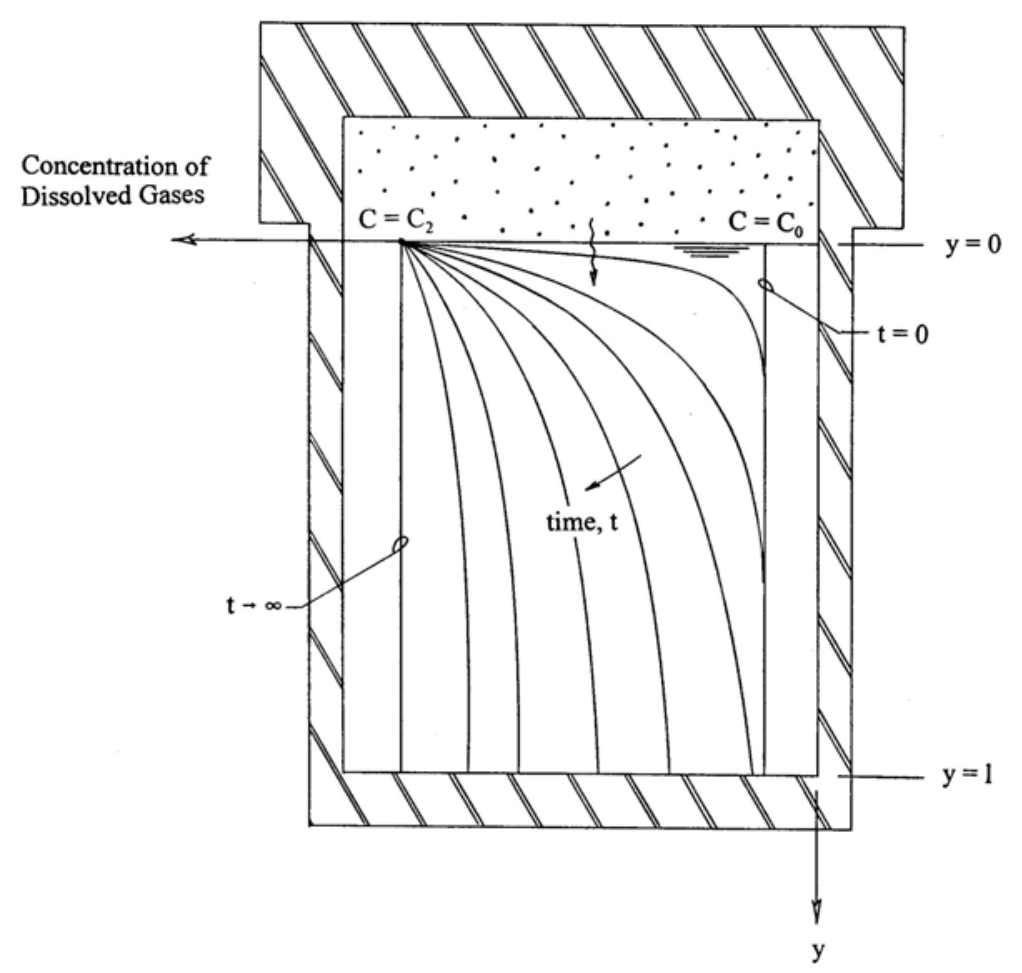

Fig. A1. Diffusion Model for Headspace-Gas Solubility 


$$
\frac{\partial C}{\partial t}=D \frac{\partial^{2} C}{\partial y^{2}}
$$

This equation is subject to the conditions that

$$
\begin{aligned}
& C=C_{0} \text { at } t=0 \text { for all values of } y \\
& C=C_{2} \text { at } y=0 \text { for all values of } t \\
& C \rightarrow C_{2} \text { as } t \rightarrow \infty \text { for all values of } y
\end{aligned}
$$

A non-dimensional, normalized gas concentration in the liquid can be defined as

$$
\theta=\frac{C-C_{0}}{C_{2}-C_{0}}
$$

In terms of this variable, the boundary conditions become

$$
\begin{array}{ll}
\theta=0 & \text { at } t=0 \text { for all } y \\
\theta=1 & \text { at } y=0 \text { for all } t \\
\theta \rightarrow 1 & \text { at } t \rightarrow \infty \text { for all } y
\end{array}
$$

The analogous heat-transfer problem is one of conduction in a slab of given thickness, initially at uniform temperature and starting at time zero one face is held constant at a new temperature, with the opposite face perfectly insulated. The solution to this classic problem is given in Ref. 12 and an adaptation is plotted in Fig. A2. Since the heat-diffusion equation is analogus to the massdiffusion equation, the solution applies as well to the present problem. The approach to the new equilibrium value is asymptotic, but the figure shows that about $98 \%$ of the eventual change has occurred when the quantity $D t / l^{2}$ reaches the value of about 1.5 . The diffusion coefficient for nitrogen diffusing through water at room temperature is about $1.64 \mathrm{e}-9 \mathrm{~m}^{2} / \mathrm{s}$ (Ref. 13). So the time required to reach $98 \%$ of the new equilibrium value is

$$
t=1.5 \frac{l^{2}}{D}=1.5 \frac{(0.361 \mathrm{~m})^{2}}{1.64 E-9 \mathrm{~m}^{2} / \mathrm{s}}=1.192 E+8 \mathrm{~s}
$$




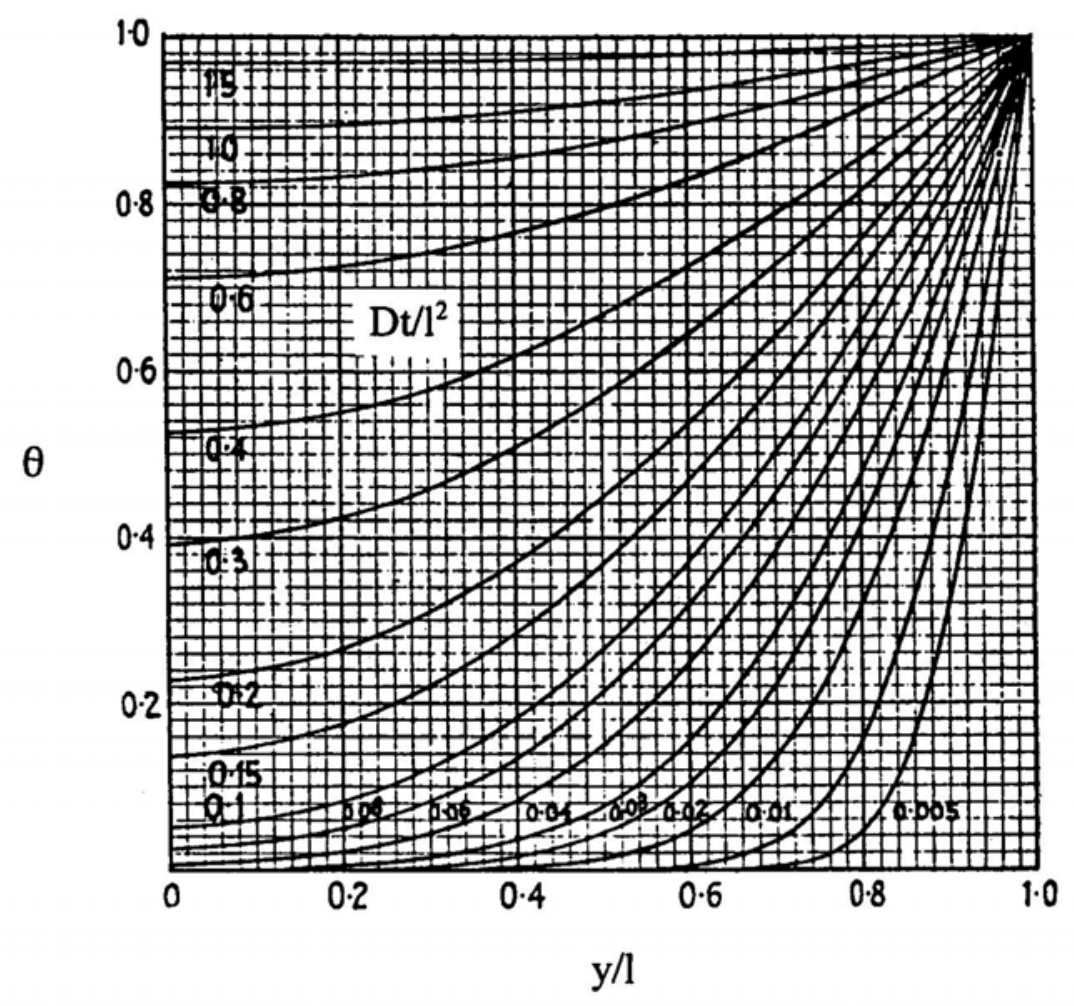

Fig. A2. Solution to the Pure Diffusion Model

That is, about 3.8 years! Even for the dissolved-gas concentration to reach, on average, only $50 \%$ of the new equilibrium concentration the solution shows that a value for $D t / l^{2}$ of about 0.25 is required, i.e., approximately 7.5 months.

\section{A2. Diffusion/Convection Model}

Transport in the GGE test containers will not be simply by diffusion, but, instead, will be augmented by the thermally induced natural convection. This will enhance the rate of movement of dissolved gas through the brine compared to that in the pure diffusion analysis presented above. Therefore, before gas solubility can be dismissed, the transport of gas via convection in the brine needs to be assessed. This will be a far more realistic solution than was presented in Section A1.

This can be estimated as follows. The discussion of natural convection within the brine/waste (Section 8.1) can be interpreted as a core of well-mixed fluid capped above and below by a thin layer of liquid across which all heat transport is by conduction. The same model can be applied to the transport of gas into, out of, and within the brine, since thermal buoyancy generates the natural convection. These same convective currents will be responsible for transport of the gas dissolved in the brine. Therefore, the "conduction layer" that pertains to the thermal problem will apply as well to the mass-transport analysis. This conduction-layer "thickness" can be estimated from the thermal model depicted in Fig. A3, which is similar to Fig. 15. The surface is at 
temperature $T_{2}$, and the concentration of gas, in moles per unit volume, dissolved just at the surface of the brine is $C_{2}$. The heat-transfer rate is augmented by the natural convection. This can be represented by the use of an "effective" value for the thermal-conductivity coefficient, $k_{\text {eff }}$, defined such that the rate of heat transfer across the brine is

$$
q=\frac{k_{e f f}}{l}\left(T_{2}-T_{3}\right)
$$

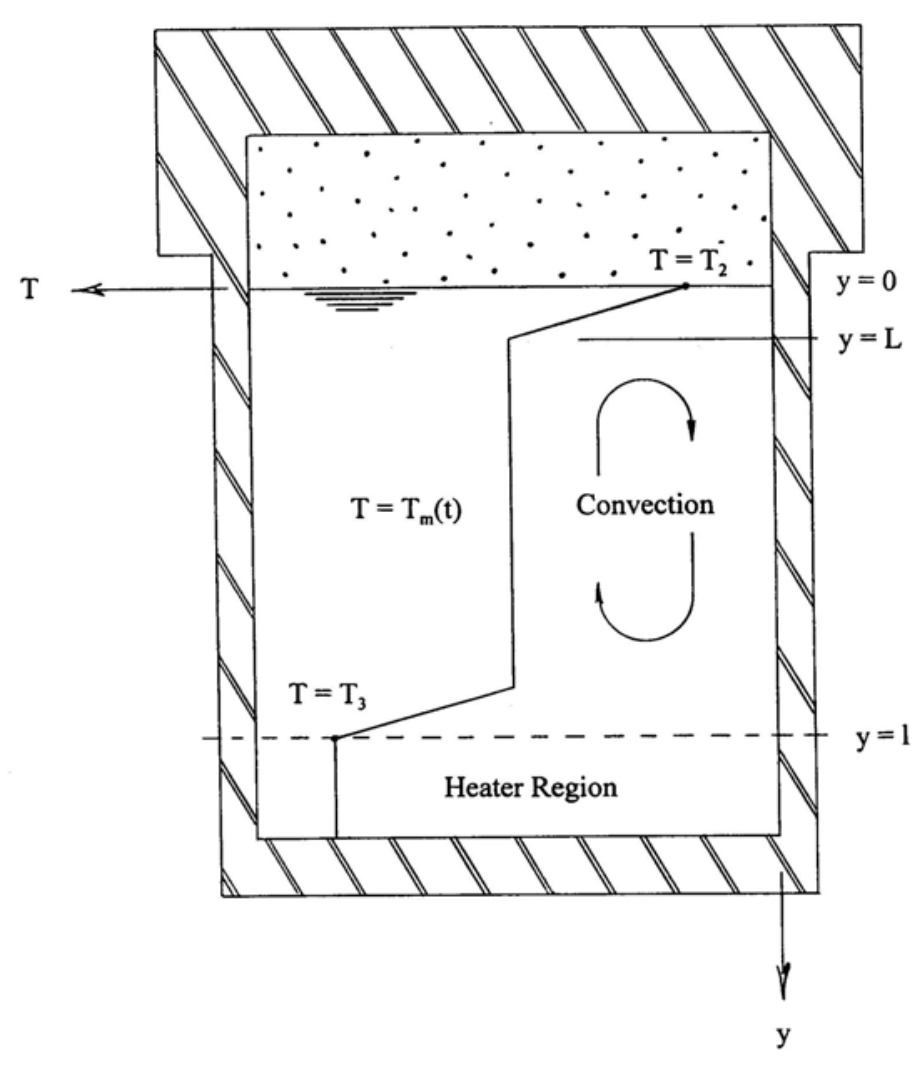

Fig. A3. Heat-Conduction and Convection Model for Determining the Temperature

The coefficient of thermal conductivity for molecular transport is $k$. Clearly, $k_{\mathrm{eff}} / k$ is greater than or equal to unity, where unity is the value if there were only molecular conduction throughout the brine.

The heat is presumed to be transferred across the "conduction" depth $L$ to the bulk fluid at temperature $T_{\mathrm{m}}$ (the "mean" temperature) only by molecular conduction. Therefore, if the heat capacity of the thin "conduction layer" is ignored, the heat flux at the brine surface, denoted by the zero subscript, is

$$
q_{0}=-\left.k \frac{d T}{d y}\right|_{y=0} \approx-\left.k \frac{\Delta T}{\Delta y}\right|_{y=0}=-k \frac{\left(T_{m}-T_{2}\right)}{L}
$$


Since the heat fluxes in Equations (A5) and (A6) must be equal, the "conduction thickness" can be found by equating these expressions, yielding

$$
L=\frac{1}{2} \frac{l}{\left[k_{e f f} / k\right]}
$$

The ratio $k_{\text {eff }} / k$ from experimental data in the literature is shown in Fig. A4 a function of the Raliegh number (14), itself a product of the Grashoff and Prandtl numbers:

$$
R a=G r \cdot P r=\frac{g \beta \Delta T l^{3}}{v^{2}} \cdot \frac{v}{\kappa}
$$

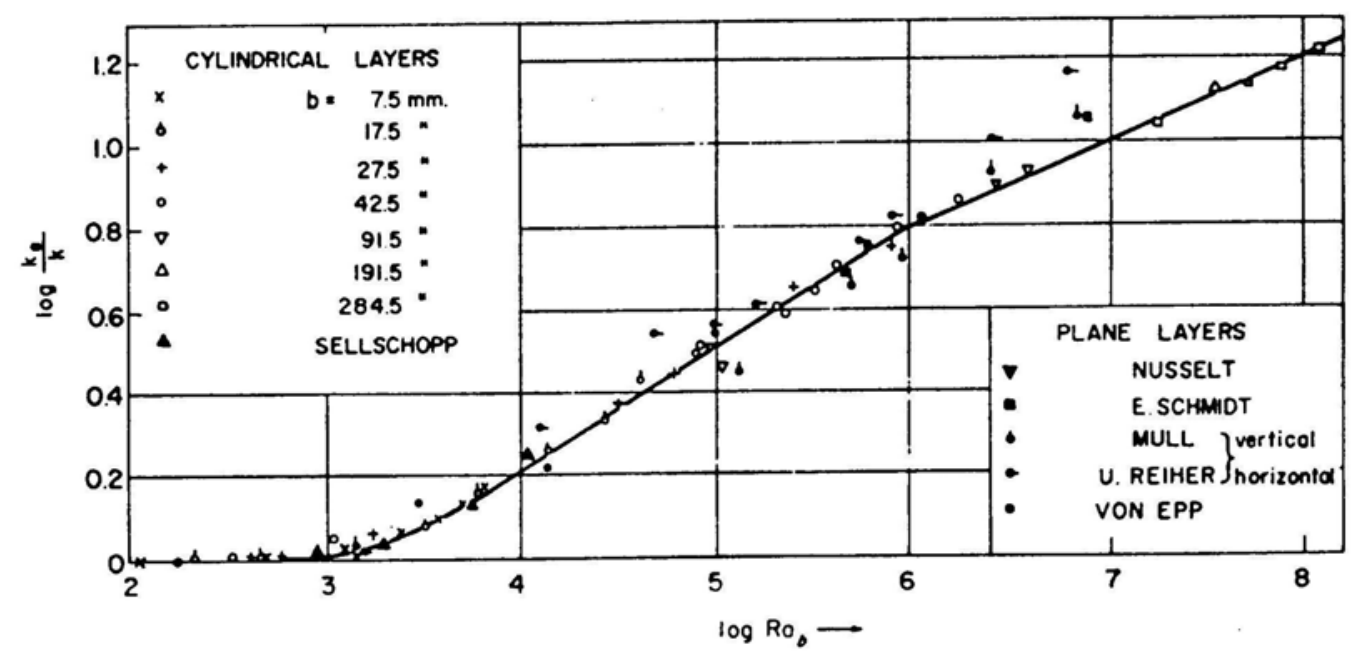

Equivalent heat conductivity for free convection through fluid layers. From H. Kraussold, Forsch. Gebiete Ingenieurw., $5: 83$ (1984).]

Fig. A4. Enhancement to the Thermal Conductivity to Account for Natural Convection

The Grashoff number is a ratio of buoyancy-induced forces (natural convection) to the viscous forces, and the Prandtl number relates viscous and thermal inertial terms. Therefore, the product of these two numbers is indicative of how natural convection of heat compares to the molecular conduction of heat. Taking the viscosity and thermal diffusivity of the brine to be those of water, with the test-container brine depth of about $361 \mathrm{~mm}$ gives a Raliegh number of about $1.4 \mathrm{e}+9$. Extrapolating Fig. A4 suggests the ratio of effective thermal conductivity to the molecular value is

$$
\frac{k_{e f f}}{k} \approx 28.2
$$


Now the assumption is made that gas is transported across the layer $L$ only by molecular diffusion. The concentration of dissolved gas at the surface is suddenly raised from the initial equilibrium value of $C_{0}$ to the new value, $C_{2}$. Upon reaching depth $L$, the gas is then distributed uniformly throughout the bulk volume by the thermally driven natural convection. Fig. A5 depicts this model. Note that the natural convection is constant (assuming external conditions do not change) since it is created and sustained by the steady temperature distribution throughout the system. In contrast, the mass transport is transient, since the quantity of dissolved gas is changing constantly from the initial molar concentration of $C_{0}$ and approaching the new equilibrium concentration, $C_{2}$. The molar flux of gas into the brine at the surface at any time, $\mathrm{t}$, is

$$
\dot{N}_{g}(t)=-\left.D \frac{d C}{d y}\right|_{y=0}
$$

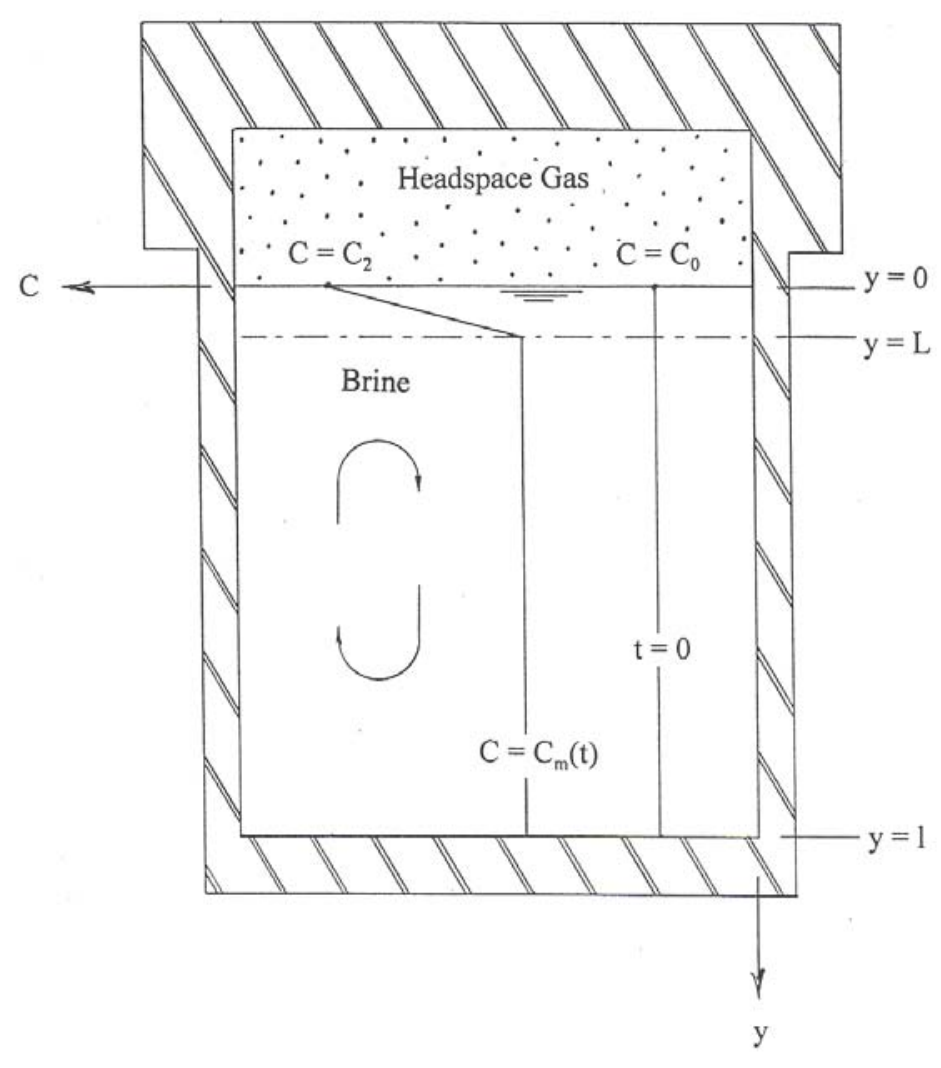

Fig. A5. Diffusion/Convection Model for Gas Solubility in the Brine

Referring to Fig. A5 (and, as was done with the thermal model, neglecting the gas quantity dissolved within the thin "diffusion layer”), this can be written as

$$
\dot{N}_{g}(t)=-D \frac{\left[C_{m}(t)-C_{2}\right]}{L}
$$


Now a balance is made on the quantity of gas dissolved. Over an infinitesimally short time interval, $d t$, the increase in the quantity of gas dissolved in the brine (moles) equals the quantity of gas (moles) transported into the brine during this time interval, or

$$
d N_{g}=\dot{N}_{g}(t) A_{c} d t
$$

In Eqn. (A11) $A_{\mathrm{c}}$ is the brine surface area (the cross-sectional area of the test container). This gives a differential equation that describes the quantity of gas dissolved over time. This equation can be recast in terms of the mass fraction of gas in solution, if desired. However, the solution process is much simpler when solved in terms of the dissolved-gas molar concentration, which is related to the number of moles of dissolved gas by

$$
C_{m}=\frac{N_{g}}{V_{l}}
$$

Differentiating Eqn. (A12),

$$
d N_{g}=V_{l} d C_{m}
$$

Substituting Equations (A10) and (A13) into Eqn. (A11) gives

$$
d C_{m}=-\frac{2 D}{l} \frac{k_{e f f}}{k} \frac{A_{c}}{V_{l}}\left(C_{m}-C_{2}\right) d t
$$

The quotient $A_{\mathrm{C}} / V_{\mathrm{l}}$ is just the inverse of the brine/waste depth, $l$. Rearranging gives

$$
d\left(C_{m}-C_{2}\right)=-\frac{2 D}{l^{2}} \frac{k_{e f f}}{k}\left(C_{m}-C_{2}\right) d t
$$

This can be directly integrated, with the result

$$
\log _{e}\left(\frac{C_{m}-C_{2}}{C_{0}-C_{2}}\right)=-\frac{2 D}{l^{2}}\left(\frac{k_{e f f}}{k}\right) t
$$

As before, a non-dimensional, normalized dissolved gas concentration is defined, but in this case it refers to the mean, or bulk, concentration, which is a function only of time and not of depth. This is written as $q_{\mathrm{m}}$, such that

$$
\theta m=\frac{C_{m}-C_{0}}{C_{2}-C_{0}}
$$

Clearly as time increases from zero to infinity, $q_{\mathrm{m}}$ varies as 


$$
0 \leq \theta_{m} \leq 1
$$

The solution, Eqn. (A16), can then be rewritten as

$$
\theta_{m}=1-e^{-\frac{2 D}{l^{2}}\left(\frac{k_{e f f}}{k}\right) t}
$$

Finally, this can be rearranged to give the time explicitly as a function of the normalized concentration:

$$
t=-\frac{l^{2}}{2 D\left(\frac{k_{e f f}}{k}\right)} \log _{e}\left(1-\theta_{m}\right)
$$

The same example as before, Section A1, is now evaluated. The multiplier term in Eqn. (A20) gives $1.41 \mathrm{e}+6 \mathrm{~s}$. For a mean, normalized gas-concentration change of $98 \%$, the log term is found to be -3.91. Therefore, the time required for the bulk gas concentration in the brine to achieve $98 \%$ of the ultimate new equilibrium gas concentration is

$$
t=-1.41 e+6 s(-3.91)=5.51 e+6 s \approx 64 \text { days }
$$

Similarly, for the dissolved-gas concentration to change to only $50 \%$ of the ultimate new equilibrium value requires over 11 days.

As expected, the time durations found with this analysis that includes a more realistic gastransport mechanism are much shorter than were calculated on the basis of purely diffusive transport throughout the entire brine volume, even with the relatively meager natural convection generated by temperature differences of only a few degrees Celsius. Nevertheless, for nitrogen gas, at least, the rate of change in the quantity of dissolved gas, and therefore the effect on headspace gas mass, due to gas solubility variation can be concluded to be so slow that the impact on the methodology addressed in this report is insignificant. Long before the bulk dissolved gas concentration approaches the new equilibrium value, the conditions will have changed yet again.

These results are based on the diffusion of gases in pure water. Diffusion coefficients of gases decrease somewhat with increasing salinity as shown in Ref. 11 using viscosities calculated per Ref. 16. Therefore, it should be expected that changes in the quantity of gas dissolved in brine would occur even more slowly than discussed here.

The diffusion coefficient for hydrogen gas in water is 1.6 times that for nitrogen in water (5). This shortens the times computed in this section proportionately, Eqn. (A20), but since the predominant gas is nitrogen, the conclusions with regard to solubility affecting the headspace-gas mass remain valid. 


\section{APPENDIX B}

\section{Thermal Expansion Experiments}

A special experimental apparatus was set up to determine the thermal expansion coefficient of brine in order to validate the data and the analysis methodology. These experiments are described in this appendix.

Figure B1 depicts the experiment. A closed container was immersed in a circulating, temperature-controlled bath. The internal volume of the container was calculated from careful dimensional measurements. The container was filled with the liquid under investigation (pure distilled water or brine), the heater was energized and the system was allowed to come to a steady state. The liquid level was adjusted so that the level just indicated on a graduated pipette mounted on the container. Careful temperature measurements were made in several places to enable the best estimate of the mean liquid temperature to be obtained.

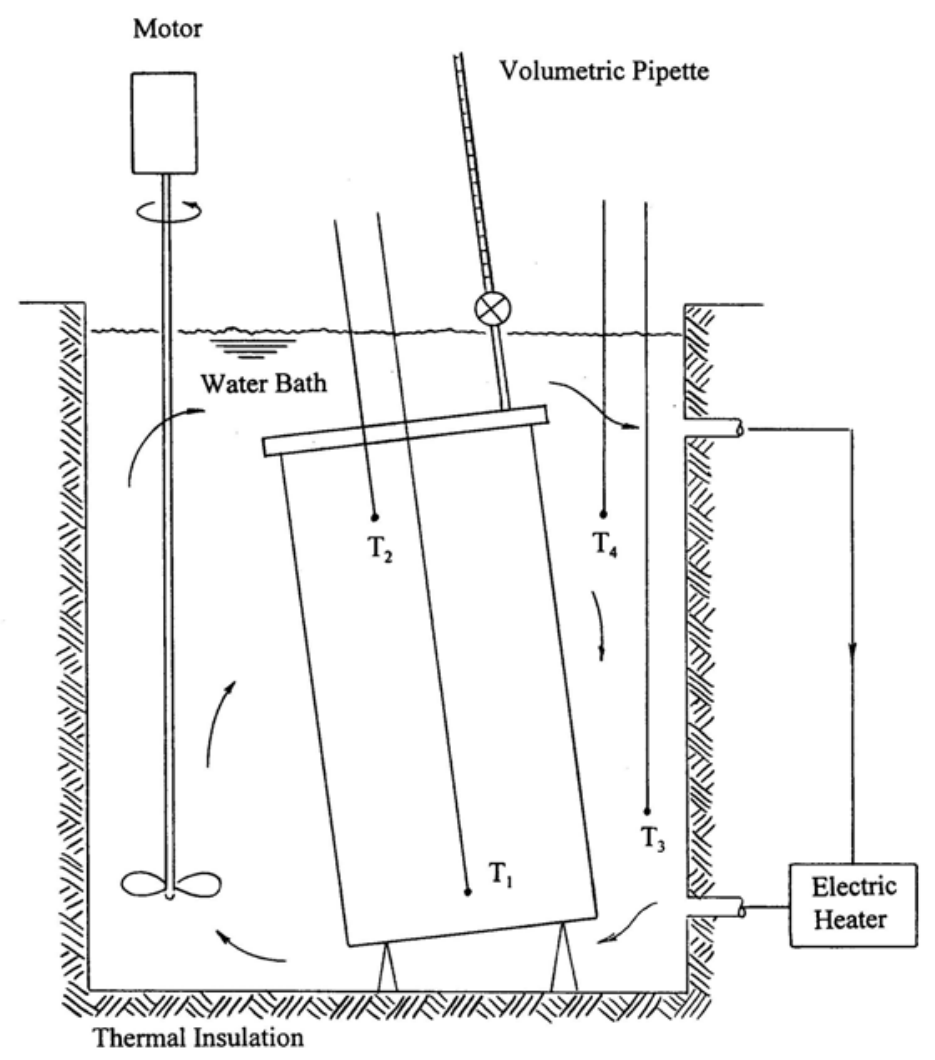

Fig. B1. Experiment Configuration for Determination of the Volumetric, Thermal-Expansion Coefficients for Pure Water and Brine

The temperature setting on the heater was then adjusted upward. Following the obtainment of new steady conditions the temperatures were recorded along with the new reading on the graduated pipette. The volume change of the test container itself as a result of the new temperature was estimated. Since the quantity of liquid within the container and the pipette was 
constant, an expansion coefficient could be calculated from the pipette reading and the temperature map. Photographs of the experimental apparatus are shown in Fig. B2_Fig. B4.

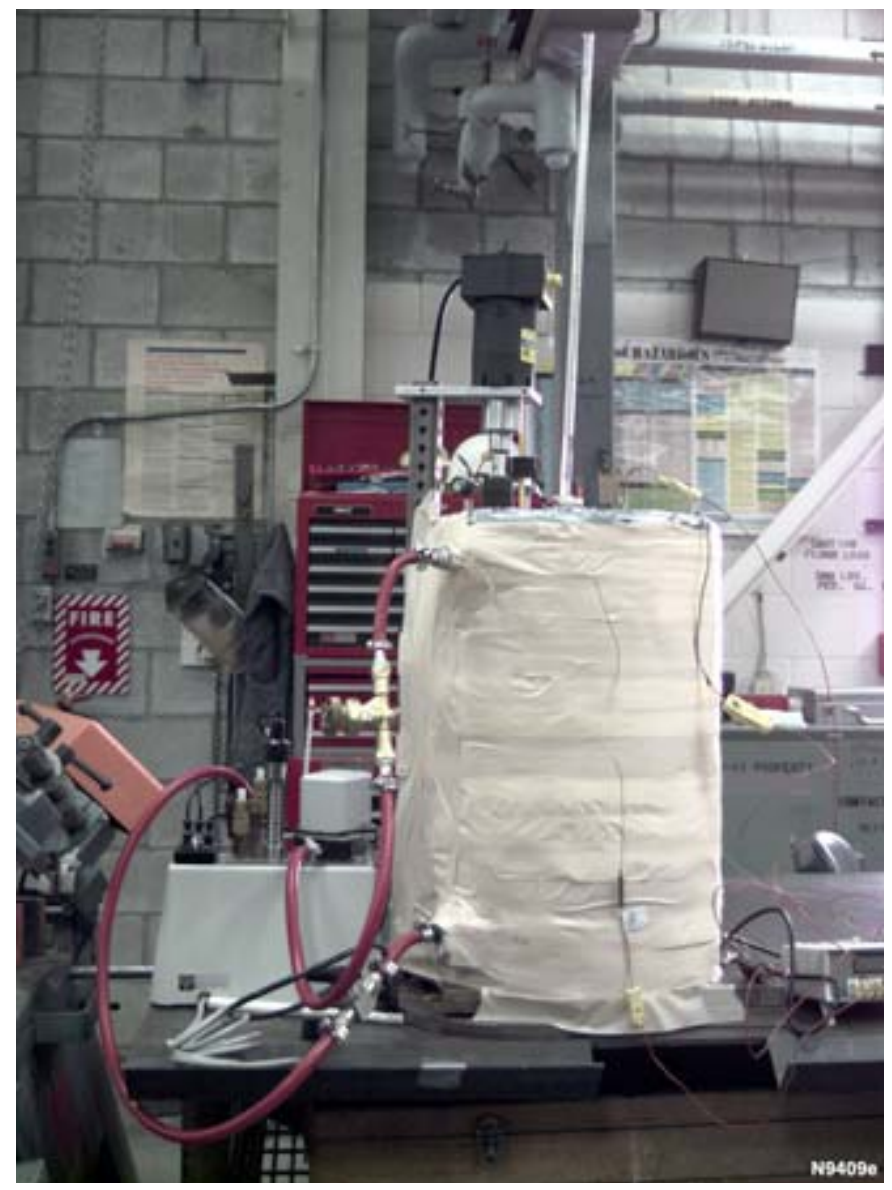

Fig. B2. Experiment Apparatus for Determination of the Volumetric, Thermal-Expansion Coefficient 


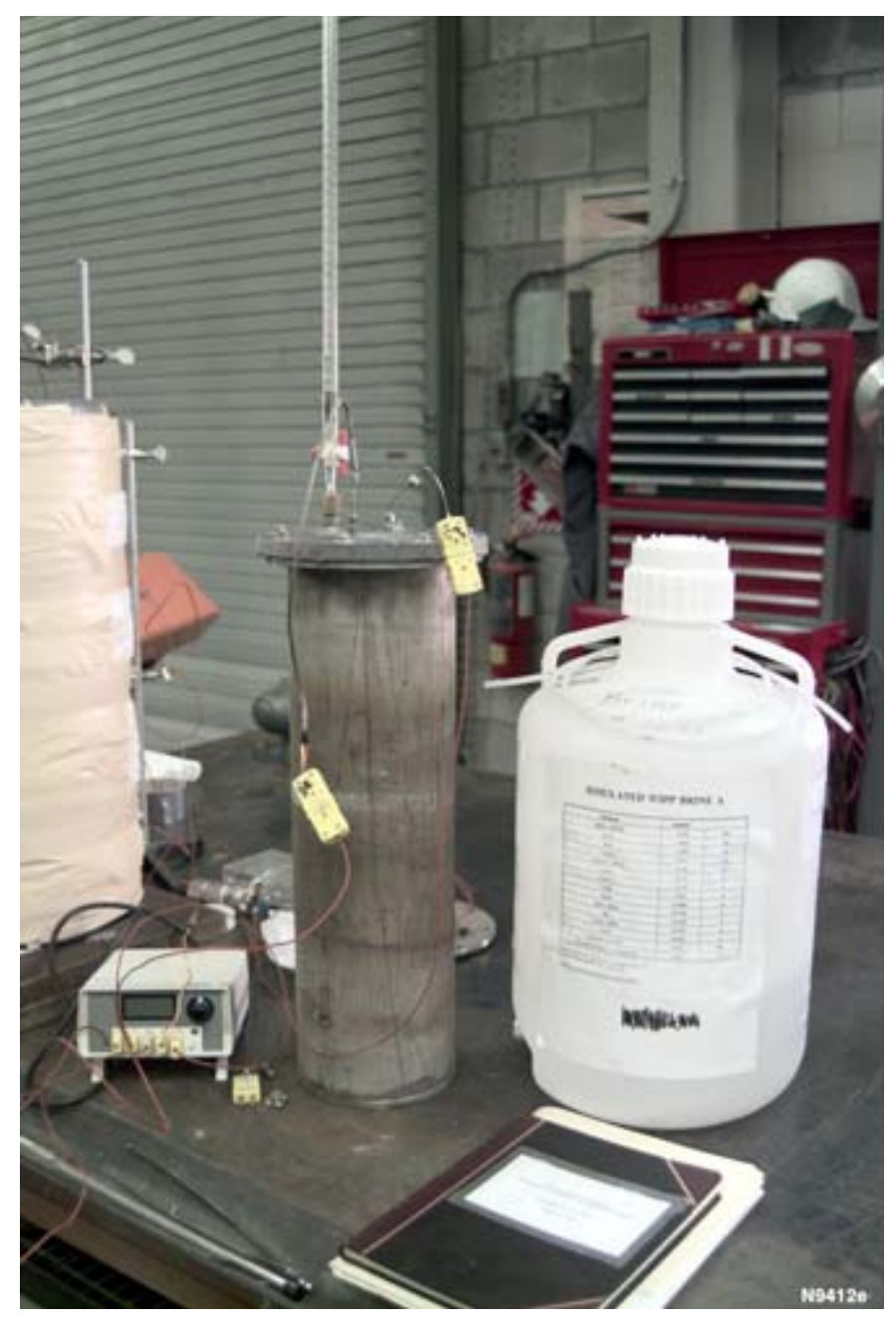

Fig. B3. Test Vessel for the Thermal-Expansion Measurements 


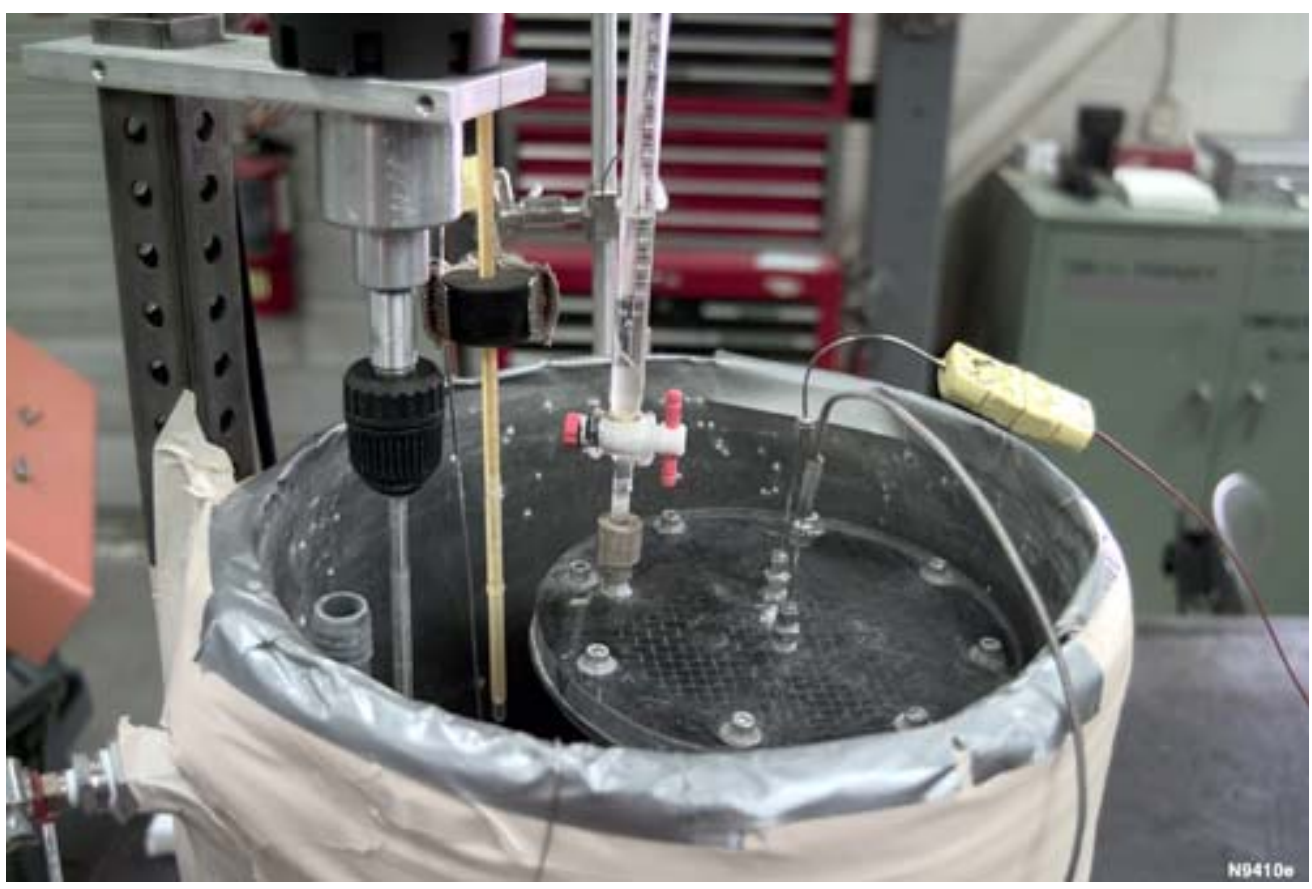

Fig. B4. Top View of the Test Vessel in its Water Bath

The apparatus was first used to check data on pure water against expansion coefficients that could be deduced from the saturated-water data in the steam tables (1). It was during these experiments that the strong influence of residual bubbles in the liquid was noted. The test vessel lid was made of a transparent material that allowed bubbles to be observed. To overcome these problems, the container was drained, very thoroughly cleaned and refilled very slowly. The container was also tilted slightly to ensure that any gas bubbles had an easy path to escape from the pipette that was mounted on the vessel lid as close to the edge as possible. After filling, the apparatus was allowed to sit for several days to release all of the bubbles. Figure B5 shows the data for water plotted against values deduced from the steam tables for temperatures within the range of interest to GGE and for both local atmospheric pressure, $85 \mathrm{kPa}$ (12.33 psia), and an elevated pressure of $14.8 \mathrm{MPa}$ (2147 psia), typical of the GGE pressures. The experimental apparatus could only be operated at atmospheric pressure. The agreement of the experimental data to that deduced from published information was found to be excellent. This provided the confidence that high-quality measurements could be made on pure brine with this apparatus.

Brine identical to that used in the GGE experiments was loaded into the test apparatus. A series of data points were generated at different temperatures. The results are shown in Figure B6. 


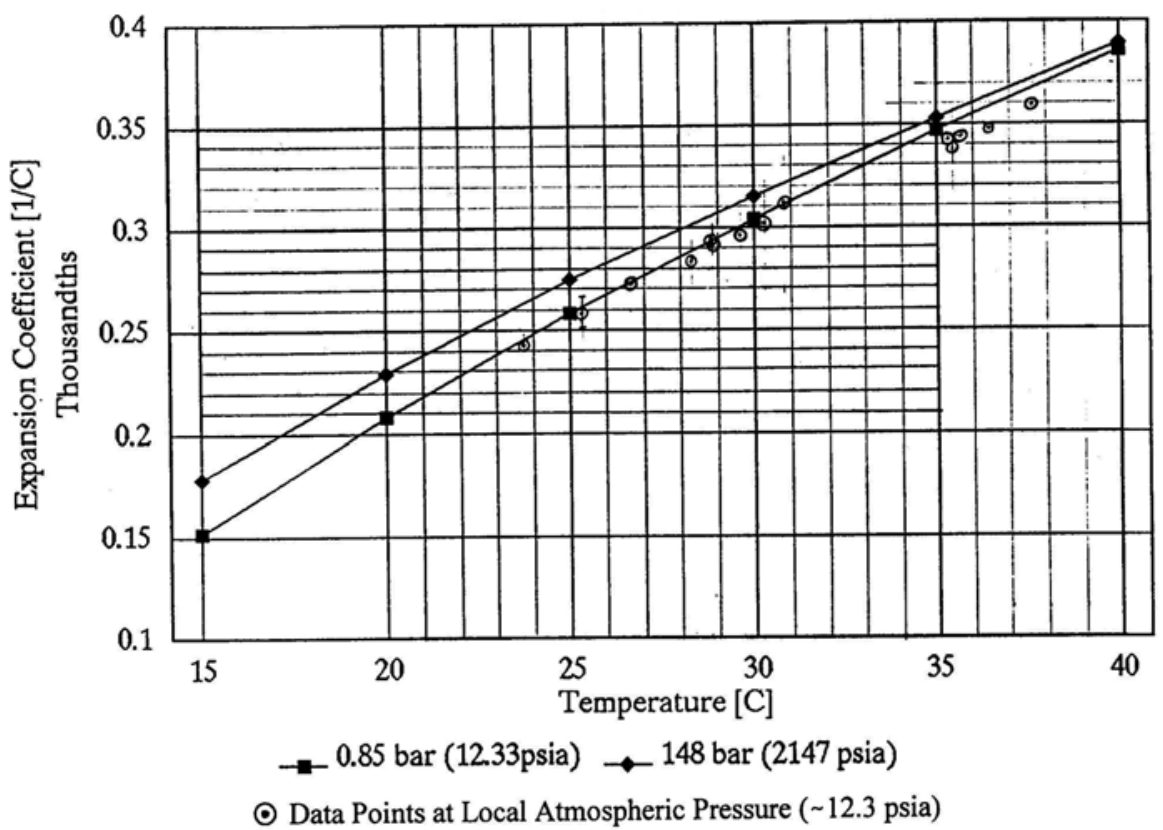

Fig. B5. Measured Volumetric Coefficients of Thermal-Expansion for Pure Water

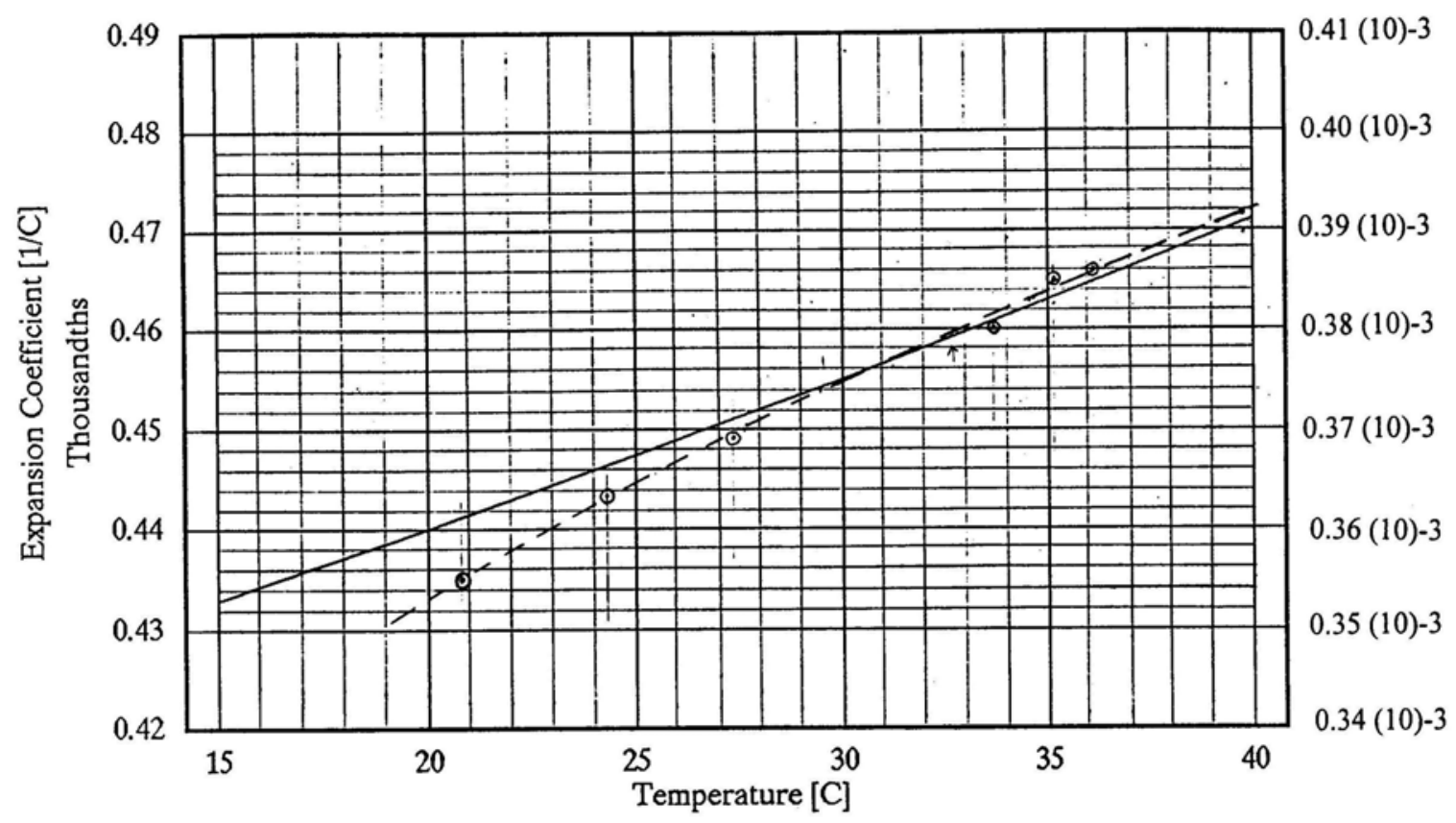

McTigue, SNL, prediction at 14.696 psia, left-hand scale

- $\odot-$ Experiment data at local atmospheric pressure ( 12.3 psia), right-hand scale

Fig. B6. Measured Volumetric Coefficients of Thermal-Expansion for Brine A 
An early intent had been to repeat these experiments with measurements on brine/waste mixtures. The difficulties encountered with bubbles during the initial measurements with water indicated quite clearly that any attempts to make measurements on brine/waste would be subject to large errors.

Data-reduction sheets for one data point shown in each of Fig. B5 and Fig. B6 are provided at the end of this appendix. 


\section{ATTACHMENT TO APPENDIX B}

Sheet 1 of 2

EXPERIMENTAL-MEASUREMENT DATA REDUCTION

$\begin{array}{lll}\text { Waste/Brine: Pure Water } & \text { mo-day- yr } & \text { hr:min } \\ \text { Date/Time of Initial Data: } & \frac{10-07-98}{10}-07-98 & \frac{11}{14}: \frac{32}{30} \\ \text { Date/Time of New Data: } & \text { 10 }\end{array}$

1. Waste/Brine Temperature

Spatial Average

$$
\begin{aligned}
& \mathrm{T}_{1-0}=0.5 *\left[\mathrm{~T}_{1 \text {-upper }}+\mathrm{T}_{1-1 \text { ower }}\right]_{0}=0.5 *[28.0+27.65]=\underline{27.825 \mathrm{C}} \\
& \mathrm{T}_{1}=0.5 *\left[\mathrm{~T}_{1-\text { upper }}+\mathrm{T}_{1-1 \text { ower }}\right]=0.5 *[34.0+\underline{33.45}]=\underline{33.725 \mathrm{C}} \\
& \Delta \mathrm{T}_{1}=\mathrm{T}_{1}-\mathrm{T}_{1-0}=33.725-\underline{27.825}=5.90 \mathrm{C}
\end{aligned}
$$

Change-Averaged Temperature

$$
\mathrm{T}_{1 \text {-change ave }}=0.5 *\left[\mathrm{~T}_{1-0}+\mathrm{T}_{1}\right]=0.5 *[\underline{27.825}+\underline{33.725}]=\underline{30.775 \mathrm{C}}
$$

2. Bath Temperature - Ave.

$$
\begin{aligned}
& \mathrm{T}_{\mathrm{b}-0}=0.5 *\left[\mathrm{~T}_{\mathrm{b} \text {-upper }}+\mathrm{T}_{\mathrm{b}-\text { lower }}\right]_{0}=0.5 *[\underline{27.75}+\underline{27.95}]=\underline{27.85 \mathrm{C}} \\
& \mathrm{T}_{\mathrm{b}}=0.5 *\left[\mathrm{~T}_{\mathrm{b} \text {-upper }}+\mathrm{T}_{\mathrm{b} \text {-1ower }}\right]=0.5 *[\underline{33.9}+\underline{34.05}]=\underline{33.975 \mathrm{C}}
\end{aligned}
$$

3. Container Temperature - Ave.

$$
\begin{aligned}
& \mathrm{T}_{\mathrm{c}-0}=0.5 *\left[\mathrm{~T}_{\mathrm{b}-0}+\mathrm{T}_{1-0}\right]=0.5 *[27.85+\underline{27.825}]=27.838 \mathrm{C} \\
& \mathrm{T}_{\mathrm{c}}=0.5 *\left[\mathrm{~T}_{\mathrm{b}}+\mathrm{T}_{1}\right]=0.5 *[\underline{33.975}+\underline{33.725}]=\underline{33.85} \mathrm{C}
\end{aligned}
$$

4. Container Ave. Temperature Change

$$
\Delta T_{c}=T_{c}-T_{c-0}=33.85-\underline{27.838}=6.01 C
$$

5. Container Volume Change

$$
\begin{aligned}
\Delta \mathrm{V}_{\mathrm{c}} & =3 \alpha \mathrm{V}_{\mathrm{c}-0} \Delta \mathrm{T}_{\mathrm{c}}=3 * 17.3 \mathrm{e}-61 / \mathrm{C} * 8944 \mathrm{ml} * 6.01 \mathrm{C} \\
& =2.79 \mathrm{ml}
\end{aligned}
$$

6. Pipette Volume Change

$$
\begin{aligned}
& \Delta \mathrm{V}_{\mathrm{p}}=\mathrm{V}_{\mathrm{p}}-\mathrm{V}_{\mathrm{p}-0}=\left(\mathrm{V}_{\mathrm{p} \text {-tare }}+50.0-\mathrm{V}_{\mathrm{p} \text {-reading }}\right)- \\
& \left(\mathrm{V}_{\mathrm{p} \text {-tare }}+50.0-\mathrm{V}_{\mathrm{p} \text {-reading }}\right)_{0} \\
& =[5.2+50.0-\underline{32.3}]-[5.2+50.0-46.0] \mathrm{ml} \\
& =13.70 \mathrm{ml}
\end{aligned}
$$


7. Waste/Brine Volume Change

$$
\Delta V_{1}=\Delta V_{c}+\Delta V_{p}=2.79+13.70 \mathrm{ml}=16.49 \mathrm{ml}
$$

8. Waste/Brine volume

$$
\begin{aligned}
& \mathrm{V}_{1-0}=\mathrm{V}_{\mathrm{c}-0}+\mathrm{V}_{\mathrm{p}-0}=\mathrm{V}_{\mathrm{c}-0}+\left(\mathrm{V}_{\mathrm{p} \text {-tare }}+50.0-\mathrm{V}_{\mathrm{p} \text {-reading }}\right)_{0} \\
& =8944+5.2+(50.0-46.0)=8953.2 \mathrm{ml} \\
& \mathrm{V}_{1}=\mathrm{V}_{1-0}+\Delta \mathrm{V}_{1}=8953.2+16.49=8969.7 \mathrm{ml}
\end{aligned}
$$

9. Expansion Coefficient at the change-averaged waste/brine temperature, $\mathrm{T}_{1 \text {-change ave }}=30.775 \mathrm{C}$ :

$\beta=\ln \left(\mathrm{V}_{1} / \mathrm{V}_{1-0}\right) / \Delta \mathrm{T}_{1}$

$=\ln (8960.7 / 8953.2) / 5.90 \mathrm{C}$

$=3.12(\omega)^{-4} 1 / \mathrm{C}$ 
EXPERIMENTAL-MEASUREMENT DATA REDUCTION

Waste/Brine: Brine-A

$\begin{array}{lll} & \text { mo-day-yr } & \mathrm{hr}: \mathrm{min} \\ \text { Date/Time of Initial Data: } & \frac{10-14-98}{10}-14-98 & \underline{08}: \frac{22}{11}: \underline{35} \\ \text { Date/Time of New Data: } & \underline{14} & \end{array}$

1. Waste/Brine Temperature

Spatial Average

$$
\begin{aligned}
& \mathrm{T}_{1-0}=0.5 *\left[\mathrm{~T}_{1-\text { upper }}+\mathrm{T}_{1-1 \text { ower }}\right]_{0}=0.5 *[30.1+30.0]=30.05 \mathrm{C} \\
& \mathrm{T}_{1}=0.5 *\left[\mathrm{~T}_{1 \text {-upper }}+\mathrm{T}_{1-1 \text { ower }}\right]=0.5 *[37.35+37.1]=37.225 \mathrm{C} \\
& \Delta \mathrm{T}_{1}=\mathrm{T}_{1}-\mathrm{T}_{1-0}=37.225-\underline{30.05}=7.175 \mathrm{C}
\end{aligned}
$$

Change-Averaged Temperature

$$
T_{1-\text { change ave }}=0.5 *\left[T_{1-0}+T_{1}\right]=0.5 *[\underline{30.05}+\underline{37.225}]=\underline{33.638 \mathrm{C}}
$$

2. Bath Temperature - Ave.

$$
\begin{aligned}
& \mathrm{T}_{\mathrm{b}-0}=0.5 *\left[\mathrm{~T}_{\mathrm{b} \text {-upper }}+\mathrm{T}_{\mathrm{b} \text {-1ower }}\right]_{0}=0.5 *[30.3+30.05]=30.175 \mathrm{C} \\
& \mathrm{T}_{\mathrm{b}}=0.5 *\left[\mathrm{~T}_{\mathrm{b} \text {-upper }}+\mathrm{T}_{\mathrm{b} \text {-1 lower }}\right]=0.5 *[37.4+37.4]=37.4 \mathrm{C}
\end{aligned}
$$

3. Container Temperature - Ave.

$$
\begin{aligned}
& \mathrm{T}_{\mathrm{c}-0}=0.5 *\left[\mathrm{~T}_{\mathrm{b}-0}+\mathrm{T}_{1-0}\right]=0.5 *[30.175+30.05]=30.113 \mathrm{C} \\
& \mathrm{T}_{\mathrm{c}}=0.5 *\left[\mathrm{~T}_{\mathrm{b}}+\mathrm{T}_{1}\right]=0.5 *[37.4+\underline{37.225}]=\underline{37.313 \mathrm{C}}
\end{aligned}
$$

4. Container Ave. Temperature Change

$$
\Delta T_{c}=T_{c}-T_{c-0}=\underline{37.313}-\underline{30.113}=\underline{7.20} \mathrm{C}
$$

5. Container Volume Change

$$
\begin{aligned}
\Delta \mathrm{V}_{\mathrm{c}} & =3 \alpha \mathrm{V}_{\mathrm{c}-0} \Delta \mathrm{T}_{\mathrm{c}}=3 * 17.3 \mathrm{e}-61 / \mathrm{C} * 8944 \mathrm{ml} * 7.20 \mathrm{C} \\
& =3.34 \mathrm{ml}
\end{aligned}
$$

6. Pipette Volume Change

$$
\begin{aligned}
\Delta \mathrm{V}_{\mathrm{p}}= & \mathrm{v}_{\mathrm{p}}-\mathrm{v}_{\mathrm{p}-0}= \\
& \left(\mathrm{V}_{\mathrm{p} \text {-tare }}+50.0-\mathrm{V}_{\mathrm{p} \text {-reading }}\right)- \\
& \left(\mathrm{V}_{\mathrm{p} \text {-tare }}+50.0-\mathrm{v}_{\mathrm{p} \text {-reading }}\right)_{0} \\
= & {[5.2+50.0-\underline{23.8}]-[5.2+50.0-44.9] \mathrm{ml} } \\
= & 21.10 \mathrm{ml}
\end{aligned}
$$


7. Waste/Brine Volume Change

$$
\Delta \mathrm{V}_{1}=\Delta \mathrm{V}_{\mathrm{c}}+\Delta \mathrm{V}_{\mathrm{p}}=3.34+21.10 \mathrm{ml}=24.44 \mathrm{ml}
$$

8. Waste/Brine Volume

$$
\begin{aligned}
& \mathrm{V}_{1-0}=\mathrm{V}_{\mathrm{c}-0}+\mathrm{V}_{\mathrm{p}-0}=\mathrm{V}_{\mathrm{c}-0}+\left(\mathrm{V}_{\mathrm{p}-\text { tare }}+50.0-\mathrm{V}_{\mathrm{p}-\text { reading }}\right)_{0} \\
&=8944+5.2+(50.0-44.9)=\underline{8954.3 \mathrm{ml}} \\
& \mathrm{V}_{1}=\mathrm{V}_{1-0}+\Delta \mathrm{V}_{1}=\underline{8954.3}+\underline{24.44}=\underline{8978.7 \mathrm{ml}}
\end{aligned}
$$

9. Expansion Coefficient at the change-averaged waste/brine

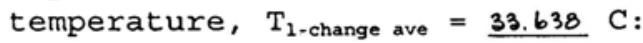

$$
\begin{aligned}
\beta & =\ln \left(\mathrm{V}_{1} / \mathrm{V}_{1-0}\right) / \Delta \mathrm{T}_{1} \\
& =\ln (\underline{8978.7} / 8954.3) / \underline{7.175 \mathrm{C}} \\
& =3.799(\omega)^{-4} 1 / \mathrm{C}
\end{aligned}
$$




\section{APPENDIX C}

\section{Local Glovebox Temperature Determination}

An attempt was made to account for the amount by which the local temperature within the glovebox exceeded the measurement recorded by the "glovebox RTD". The difference between the actual local temperature in the vicinity of the test vessel head and the temperature measured by the glovebox RTD was termed the "excess" temperature.

\section{C1. Temperature Mapping}

The glovebox RTD was mounted in its normal position underneath the subfloor and against the wall of the glovebox. An auxiliary RTD was moved around the glovebox to create a map of the temperatures for comparison to that measured by the fixed RTD. Attached to this appendix is a summary communication of the mapping effort and the results found.

Another session successively placed the auxiliary RTD in the head region between test containers. The temperature measurements from this RTD were compared to those measured by the fixed glovebox RTD. The variation in these measurements throughout the glovebox were modest, with an approximately constant deviation from the temperature measured by the glovebox RTD.

\section{C2. Excess-Temperature Algorithm}

The local temperature is actually subject to various influences: number and location of test vessel heaters that may be energized at any time; argon-gas circulation and oxygen-removal equipment function, lighting heat load, etc. The variability of these influences was not investigated. Instead, the excess temperature was assumed to be simply a function of the glovebox temperature measurements.

A number of data points from the exercises described above were plotted against the glovebox temperature measurement and a $2^{\text {nd }}$-order polynomial fit was made to the data using a Microsoft Excel plotting function. Since the experiments found that the excess temperature approaches a constant value of $2-3^{\circ} \mathrm{C}$ as the glovebox temperature approaches the test-containers' set-point value of $30^{\circ} \mathrm{C}$, a linear fit was deemed inappropriate. The basis for selecting a $2^{\text {nd }}$-order polynomial fit was that the excess temperature should be a monotonic function of the glovebox temperature (higher-order polynomials would not necessarily be monotonic).

The data points and the curve-fit are shown in Fig. C1. The equation for this curve is

$$
\Delta T_{e X}=0.0215 T_{G B}^{2}-1.2975 T_{G B}+21.503
$$

This algorithm was applied consistently to all of the archived data. 


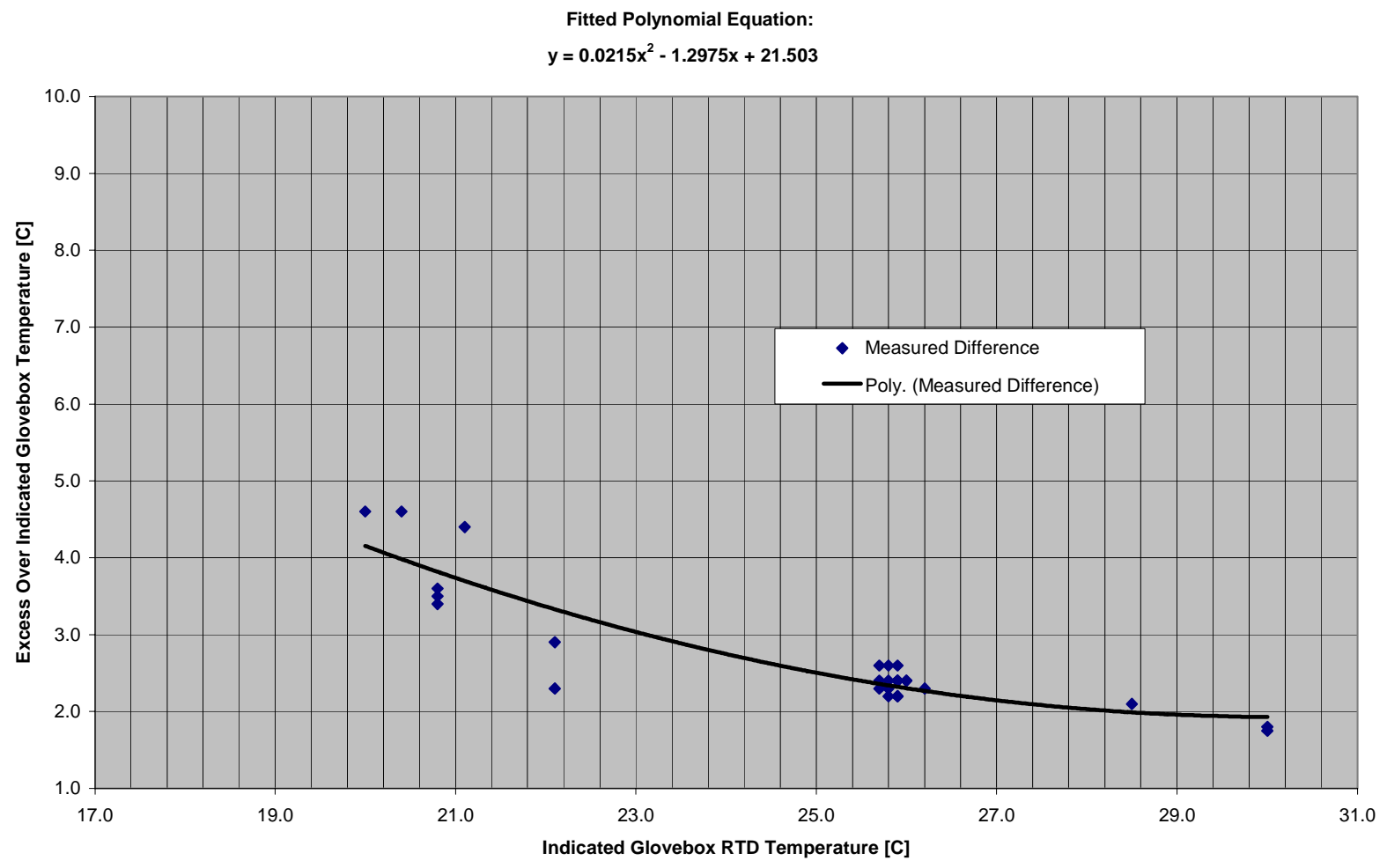

Fig. C1. Estimate of Local Test Container Temperature Excess 


\section{ATTACHMENT TO APPENDIX C}

Observations From An Impromptu Experiment- GOE

\section{FSF 9.98}

Lasry foulkrod and Frank Felicione performed an impromptu, informal experinent to determine if the "glatebox temperature" measutement was influenced by the location where it was measured. This is described below. The experiment was done on 99-03-98, between $\sim 10: 30$ to 11:30.

The system was running steadily, no gross citculation in the box, and thcre was no activity in the area. All measurements were made using the RTD's that were in place and connected to the DAS.

Temperature-Measutement Positions:

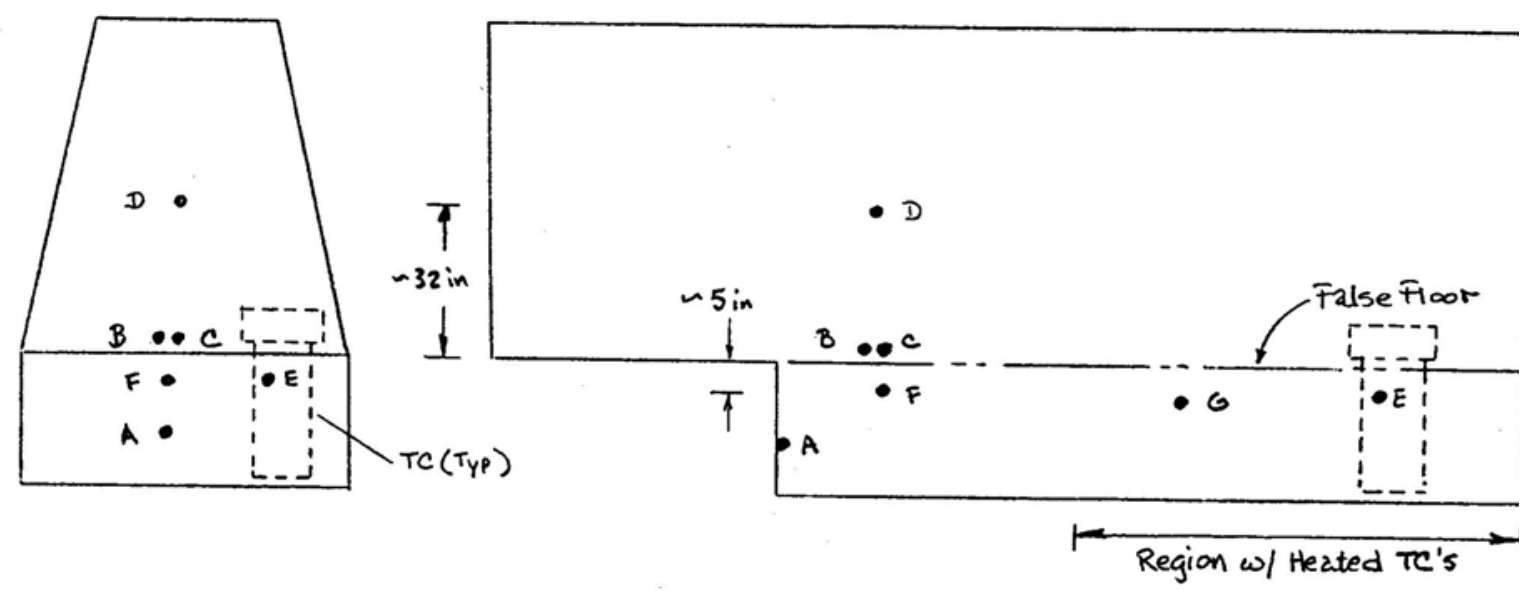

South Elevation East Elevation

$\begin{array}{lll}\text { Initial Temperatures: } & \text { A } & 25.3 \mathrm{C} \\ & B & 26.6 \\ & C & 26.8 \\ & \\ & \\ E & - & -30.5 \text { (ave., actual TC's vatied from } 30.3-30.8 \mathrm{C} \text { ) } \\ & F & - \\ G & -\end{array}$

Position A is the "glovebox temperature" measulement. There are RTD's lying on the false floor (rather haphazardly) at positions $B$ and $C$. 
Experiment:

1) Remove RTD from position A (next to wall) and place near position $B / C$ (on the false floot, within a few inches of each other). Result: $26.5 \mathrm{C}$ after $\sim 10 \mathrm{~min}$ and apparently steady.

2) Move this same RTD to position F ( the false floor as position $A$, but away from the wall). Result: $26.5 \mathrm{C}$ (ie., no change from position $B / C$ ).

3) Move this same RTD to position G. Result: $>27.2 \mathrm{C}$ (ie., not quite equilibrated after $\sim 5 \mathrm{~min}$ ).

4) Move RTD from position C to position D (hanging from hoist chain) Result: $28.1 \mathrm{C}$

Conclusions :

1) There are temperature gradients within the glovebox. These very likely result from the heat input to the TC heaters, although the ZPPR cell teniperature may also have temperature gradients within.

2) Temperature measurements at position A are affected by the wall temperature and are not a good indicator of the temperature just above the TC's. Positions $B / C$ provide a better'indication, but the temperature measured here is probably somewhat cooler than that at the same elevation (just above the false floor) but in the region where the heated $T C$ 's are located [the lead length on the RTD would not allow it to be repositioned to this area during this experiment]. 
3) The result is that theie is no historical record of the "glovebox temiperature" that is representative of the temperature in the vicinity of the upper part of the TC's. These temperatures will have to be estimated on the basis of archived data at positions $A, B, \xi C$. [ Warry Foulkrod has cautioned that the RTD's at positions $B / C$ may have not always been at these same positions!].

This will impact our ability to unravel the historical pressure variation to some extent. 
$10-15-98 \quad \sim 09: 30$

Larry foulkrod moved RTD No. 15 and 16 to positions more representative of the $G B$ environment surrounding the TC's.

Both RTD's were moved to a lateral position very near the center of the heated TC region. No. 15 was placed about 6 in above the false floor, ie., at an elevation equal to that at the top of the TC's. No. 16 was placed $\backsim 2$ in below the false floor. These are positioned along the $G B$ centerline and between $T C$ positions 05 \& 07 .

In their original positions (positions $B \dot{\varepsilon} C$ on the figure, p. 1) these both read $26.8 \mathrm{C}$. In their new positions they show

$\begin{array}{llll}\text { RID NO. } 15 & 28.0 \mathrm{C} \\ \text { RTD No. } 16 & 27.9 \mathrm{C}\end{array}$

At the same time the "GB Temperature" (position A, p.1) read $25.5 \mathrm{C}$. The TC RTD's varied from 29.9 to $30.6 \mathrm{C}$.

114 


\section{APPENDIX D}

\section{Computer Application Program}

The entire pressure-compensation methodology was formulated as an adjustment to the measured pressure, where all of the influences other than gas generation were methodically stripped out of the results. The analysis was programmed for computer application in FORTRAN. The final computer program, the result of evolutionary improvements, is titled GGE7b1.

\section{D1. Program Input}

The program may be applied to discrete data points entered manually or to a very large number of data points normally generated as an input listing from another software package. The former is useful for checkout or other special purposes; the latter "streaming-data-input" approach was used to facilitate input of the 26,000-plus data points for each test container that generated the results shown in Section 11.1.

Attachment 1 to this appendix is a listing of the input files that contain the unique information required to analyze each test container. These set-up files are all unformatted text (ASCII) file types, and are labeled

\section{SETXXX.TXT}

The $x x x$ is the identification number of the test container, e.g., SET160.TXT pertains to TC-160, making each file unique to the corresponding test container. Each SETxxx.TXT file contains all of the set-up information required to analyze the given test-container data. This includes geometry, sampling data, RTD and PXD calibration data, the temperature-step data for calculating $\beta_{\mathrm{H}}$ and $\beta_{\mathrm{L}}$, and miscellaneous physical properties.

A second group of files for the voluminous streaming data input carry the labels

\section{STRxxx.TXT}

Again, the $x x x$ refers to a test-container identification number. The STRxxx.TXT files are unformatted text files that contain the full data set (test container and glovebox RTD data and pressure data, along with some specific parameters such as local glovebox temperature excess over the measurement) for each test container for use with the streaming-input option. The STRxxx.TXT files also contain indicators of duplicate or "dropped" data (zeros). A parameter, ISTRM, in the SETxxx.TXT file triggers whether the selected data points provided in the SETxxx.TXT file are used, or whether the streaming-input data file is to be read to supply this information.

Data from the data-acquisition system were transferred to a Microsoft EXCEL-97 spreadsheet program. Logic was programmed into these spreadsheet files to remove duplicated or "dropped" data (zeros) from the raw data-point sequence numbers in the file, resulting in a "valid" sequence numbering for the data. The spreadsheet also calculated the local glovebox temperature 
algorithm (Appendix C). Unformatted text files, STRxxx.TXT, were then created from these spreadsheet files. A procedure for generating the STRxxx.TXT files is Attachment 2 to this appendix. These files are approximately 1.7 Mbytes for each test container, and consist of approximately 26,000 lines of printed data. A CD-ROM disk containing the 14 files for the GGE test containers has been placed in the GGE Project Files.

\section{D2. Specification of the Test Container to be Analyzed}

The selection of the actual test container to be analyzed by the application program is made by copying the selected SETxxx.TXT and STRxxx.TXT files, respectively, to the two identicalcontent files that are actually read by the GGE7b1 application program:

\section{GGESET.TXT \\ GGESTR.TXT}

For example, to apply the data-adjustment methodology to data from test container No. 166, the file SET166.TXT needs to be copied to GGESET.TXT and (if the streaming-data option is to be used) the file STR166.TXT needs to be copied to GGESTR.TXT. The currently specified subdirectories for GGESET.TXT and GGESTR.TXT files are specified in two OPEN statements at the beginning of the application program.

\section{D3. Program Output}

Execution of the applications program results in the generation of two output files. The first, GGE.OUT, is an unformatted text file that contains virtually all of the intermediate results, mirrors the input, etc. This file is useful for checking intermediate calculations by the program, but becomes an extremely large file when many thousands of data points are analyzed. The second output file, GGESUM.OUT, is a summary unformatted text file that contains the raw pressure measurement and the adjusted pressure, but, to keep its size reasonable, virtually no intermediate calculations. This file is used for plotting the results, and was the origin of the plots shown in Section 11.1 in this report. The specific sub-directory paths for GGE.OUT and GGESUM.OUT files are specified in the OPEN statements at the beginning of the applications program, and these could easily be changed to whatever path is desired.

\section{D4. Program Map and Listing}

The programming for the complete methodology, including the one-dimensional thermal model, was developed specifically for this application. A top-level map that describes the logic flow through the programs is provided in Attachment 3 to this appendix. The listing of the FORTRAN source program, GGE7b1, is Attachment 4 to this appendix.

\section{D5. Execution of the Program}

The program was compiled and executed on Ryan-McFarland Corporation's RM/FORTRAN, Version 02.45.02, with program linking via Phoenix Software Associates Ltd. PLINK86 linker program. The program was run on an Intel Pentium II processor, operating at $233 \mathrm{MHZ}$. 
Execution of the approximately 26,000 data points for each test container required approximately 2.25 minutes.

\section{D6. Results Plots}

The plots of the temperature, pressure, and mole fraction of generated gases were produced with the Microsoft EXCEL-97 spreadsheet program. The output GGESUM.OUT files were read into an EXCEL spreadsheet and plotted using the standard EXCEL plotting features. 


\section{ATTACHMENT 1 TO APPENDIX D}

\section{SET $x x x$. TXT Files Listings}

The following pages contain the setup parameters for use in Program GGE7b1 (SETxxx.txt files) in the following order:

$\begin{array}{lll}\text { Filename } & \text { Test Container }^{1} & \text { No. of Sheets } \\ \text { SET151.txt } & \text { TC-151 } & \text { (3 sheets) } \\ \text { SET152.txt } & \text { TC-152 } & \text { (3 sheets) } \\ \text { SET153.txt } & \text { TC-153 } & \text { (3 sheets) } \\ \text { SET160.txt } & \text { TC-160 } & \text { (3 sheets) } \\ \text { SET161.txt } & \text { TC-161 } & \text { (3 sheets) } \\ \text { SET161e.txt } & \text { TC-161e } & \text { (3 sheets) } \\ \text { SET162.txt } & \text { TC-162 } & \text { (3 sheets) } \\ \text { SET163.txt } & \text { TC-163 } & \text { (3 sheets) } \\ \text { SET164.txt } & \text { TC-164 } & \text { (3 sheets) } \\ \text { SET165.txt } & \text { TC-165 } & \text { (3 sheets) } \\ \text { SET166.txt } & \text { TC-166 } & \text { (3 sheets) } \\ \text { SET167.txt } & \text { TC-167 } & \text { (3 sheets) } \\ \text { SET168.txt } & \text { TC-168 } & \text { (3 sheets) } \\ \text { SET169.txt } & \text { TC-169 } & \text { (3 sheets) } \\ \text { SET170.txt } & \text { TC-170 } & \text { (3 sheets) }\end{array}$

1. SET161e.txt is the same as SET161.txt, but uses empirical, trial calibration factors for the brine/waste RTD (see discussion in Section 11.2.1). 


\section{ATTACHMENT 1 OF APPENDIX D}

\section{TC-151 Setup}

LENGTHS OF VARIOUS REGIONS: LO, L1, L2, L3, L4, L5, L6

$\begin{array}{lllll}0.03795 & 0.04440 & 0.04260 & 0.09070 & 0.0\end{array}$

$\odot .36154 \quad 0.36154$

ARGON GAS DATA: NAR/TAR, AKAR, VAR, CAR, RAR

\section{3}

$\begin{array}{lllll}-23.16 & 0.0152 & 1.196 \mathrm{E}-5 & 520.3 & 1.630 \\ 26.84 & 0.0177 & 1.686 \mathrm{E}-5 & 520.3 & 1.358 \\ 126.84 & 0.0223 & 2.836 \mathrm{E}-5 & 520.3 & 1.019\end{array}$

TC HEAD DATA: NHD/THD, AKHD, VHD, CHD, RHD 1

$25.0 \quad 12.55$

HEADSPACE-GAS DATA: NHS/THS, AKHS, VHS, CHS, RHS

\section{3}

$\begin{array}{llllr}-23.16 & 0.0222 & 0.778 E-7 & 1038.3 & 200.4 \\ 26.84 & 0.026 & 1.078 E-7 & 1040.8 & 167.0 \\ 126.84 & 0.0325 & 1.785 E-7 & 1045.9 & 124.9 \\ \text { BRINE/WASTE DATA: } & \text { NBW/TBW, AKBW, VBW, CBW, RBW }\end{array}$

4

$\begin{array}{llrrr}\odot . & 0.501 & 1.441 \mathrm{E}-6 & 3399.4 & 1243.6 \\ 20 . & 0.597 & 0.8162 \mathrm{E}-6 & 3393.0 & 1233.1 \\ 40 . & 0.628 & 0.5356 \mathrm{E}-6 & 3401.1 & 1221.9 \\ 60 . & 0.651 & 0.3893 \mathrm{E}-6 & 3408.0 & 1210.0\end{array}$

CYCLE LIMIT AND TEMPERATURE CONVERGENCE TOLERANCE: LIMIT, DTERR $100 \quad 0.001$

WATER-VAPOR PRESSURE TABLE

21

$120 . \quad 0.023388$

$2 \quad 21 . \quad 0.024877$

322 . 2.026447

$423 . \quad 0.028104$

$524 . \quad 0.029850$

$625 . \quad 0.031691$

$726 . \quad 0.033629$

$827 . \quad 0.035670$

928.20 .037818

1029.20 .040078

$1130 . \quad 0.042455$

$12 \quad 31 . \quad 0.044953$

$13 \quad 32 . \quad 0.047578$

14 33. 0.050335

$1534 . \quad 0.053229$

$16 \quad 35 . \quad 0.056267$ 


$\begin{array}{rrr}17 & 36 . & 0.059454 \\ 18 & 37 . & 0.062795 \\ 19 & 38 . & 0.066298 \\ 20 & 39 . & 0.069969 \\ 21 & 40 . & 0.073814\end{array}$

TEST-CONTAINER VOLUME \& LINEAR THERMAL-EXPANSION COEFFICIIENT 7272. $11.3 E-6$

GLOBAL EXCESS TEMP OF GB INTERIOR VS. THE GB RTD MEASUREMENT $\odot$.

NUMBER OF RTD \& PRESS-XDUCER CALIBRATIONS: NCALT, NCALP 86

RTD CALIBRATION INFORMATION: [DATE:HR],PT NO , COMP, RESERR

$\begin{array}{rrll}11-03-95: ? ? & \odot & \odot . & \odot . \\ 07-15-96: ? ? & 1080 & \odot . & \odot . \\ 01-13-97: ? ? & 5322 & \odot . & \odot .1 \\ 07-09-97: ? ? & 9382 & \odot . & \odot .1 \\ 11-21-97: ? ? & 11856 & \odot . & \odot . \\ 05-21-98: ? ? & 16852 & \odot . & \odot . \\ 11-23-98: ? ? & 21208 & \odot . & \odot . \\ \text { ๑5-24-99:?? } & 25371 & \odot . & \odot .\end{array}$

PRESSURE-XDUCER CALIBRATION INFO: [DATE:HR],PT NO ., COMP., RESIDP

$\begin{array}{rrrr}\text { 05-24-96:?? } & 2 & \odot . & \odot . \\ 11-14-96: ? ? & 3936 & \odot . & \odot . \\ 05-14-97: ? ? & 8065 & \odot . & \odot . \\ 12-02-97: ? ? & 12844 & \odot . & \odot . \\ 06-02-98: ? ? & 17134 & \odot . & \odot . \\ 12-07-98: ? ? & 21533 & \odot . & \odot .\end{array}$

OVERALL REFERENCE PT DATA: NPTP, PP, TRTDP, TGBP, TGBEXP

$10001 \quad 2170.1 \quad 31.0 \quad 29.3 \quad 1.94$

HS-GAS COMPRESSIBILITY TABLE DATA: NUM, VLO, VHI

$50 \quad 0.17 \quad 0.19$

BRINE MASS AND MOLECULAR WEIGHT: AMB [G],WTMOLB [G/GMOLE]

7708. $\quad 18.5$

UNIVERSAL GAS CONSTANT: RU [PSI*ML/GMOLE/K],ATM PRESS [PSIA] $1205.95 \quad 14.696$

SOL GAS INFO: VOL FRACT[VFRG], MOL WT[WTMOLG], CONSTANTS A, B, C $\begin{array}{llll}1 . & -67.3877 & 86.3213 & 24.7981\end{array}$

SAMPLE VOLUME: VSAMPL

3.05

NUMBER OF GAS-SAMPLE DATA POINTS: NSAMPL

4

GAS-SAMPLE DATA: [ID], NPTS( I ),PS1, PS2, TRTD, TGB, TGBEX

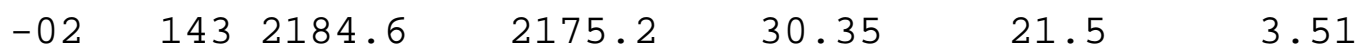

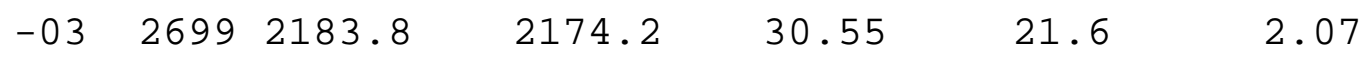

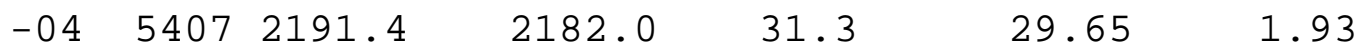

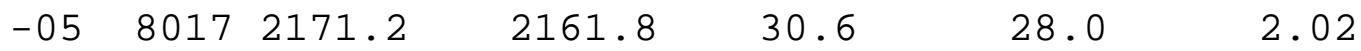


PXD BACKFILL INFO: [NO.,PT.NO.],PC1, PC2, TC1, TC2, TGBC, TGBEXC

\begin{tabular}{lrllllll}
-01 & 2 & 2128.3 & 2128.3 & 24.0 & 24.0 & 20.9 & 3.82 \\
-02 & 3936 & 2178.3 & 2181.9 & 30.7 & 30.7 & 28.3 & 2.00 \\
-03 & 8065 & 2161.8 & 2162.2 & 30.5 & 30.5 & 27.7 & 2.06 \\
-04 & 12844 & 2155.0 & 2154.4 & 30.4 & 30.4 & 22.1 & 3.33 \\
-05 & 17134 & 2153.9 & 2154.4 & 30.6 & 30.6 & 19.9 & 4.20 \\
-06 & 21533 & 2154.6 & 2156.2 & 30.2 & 30.2 & 25.9 & 2.32 \\
\multicolumn{2}{l}{ BETA } & DETERMINATION POINTS: NBETA & & &
\end{tabular}

BETA DETERMINATION POINTS: NBETA

2

FIRST (LOW TEMP) BETA-PT. REF. CONDITIONS : NPTK, PK, TRTDK, TGBK, TEXK $23075 \quad 2142.3 \quad 29.6 \quad 20.0 \quad 4.15$

BETA-POINT STEP CONDITIONS: PB, TRTDB, TGBB, TEXB

$\begin{array}{lllll}23236 & 2078.5 & 25.0 & 20.8 & 3.82\end{array}$

SECOND (HIGH TEMP) BETA-PT. REF. CONDITIONS:PK, TRTDK, TGBK, TEXK

$22098 \quad 2155.0 \quad 30.2 \quad 26.1 \quad 2.28$

BETA-POINT STEP CONDITIONS: PB, TRTDB, TGBB, TEXB

$\begin{array}{lllll}22122 & 2259.7 & 37.4 & 26.2 & 2.27\end{array}$

NUMBER OF DATA POINTS TO BE ADJUSTED, NDATA, AND INPUT KEY ISTRM $3 \quad 1$

DATA-PT NO., DATE\&TIME, PRESS, RTD TEMP, GB TEMP, EXCESS TEMP

$\begin{array}{rlllll}8001 & 05-11-97: 18 & 2170.7 & 30.7 & 28.4 & 2.0 \odot \\ 14001 & 01-20-98: 16 & 2151.7 & 30.5 & 21.0 & 3.74 \\ 20001 & 10-02-98: 09 & 2156.0 & 30.3 & 25.5 & 2.40\end{array}$




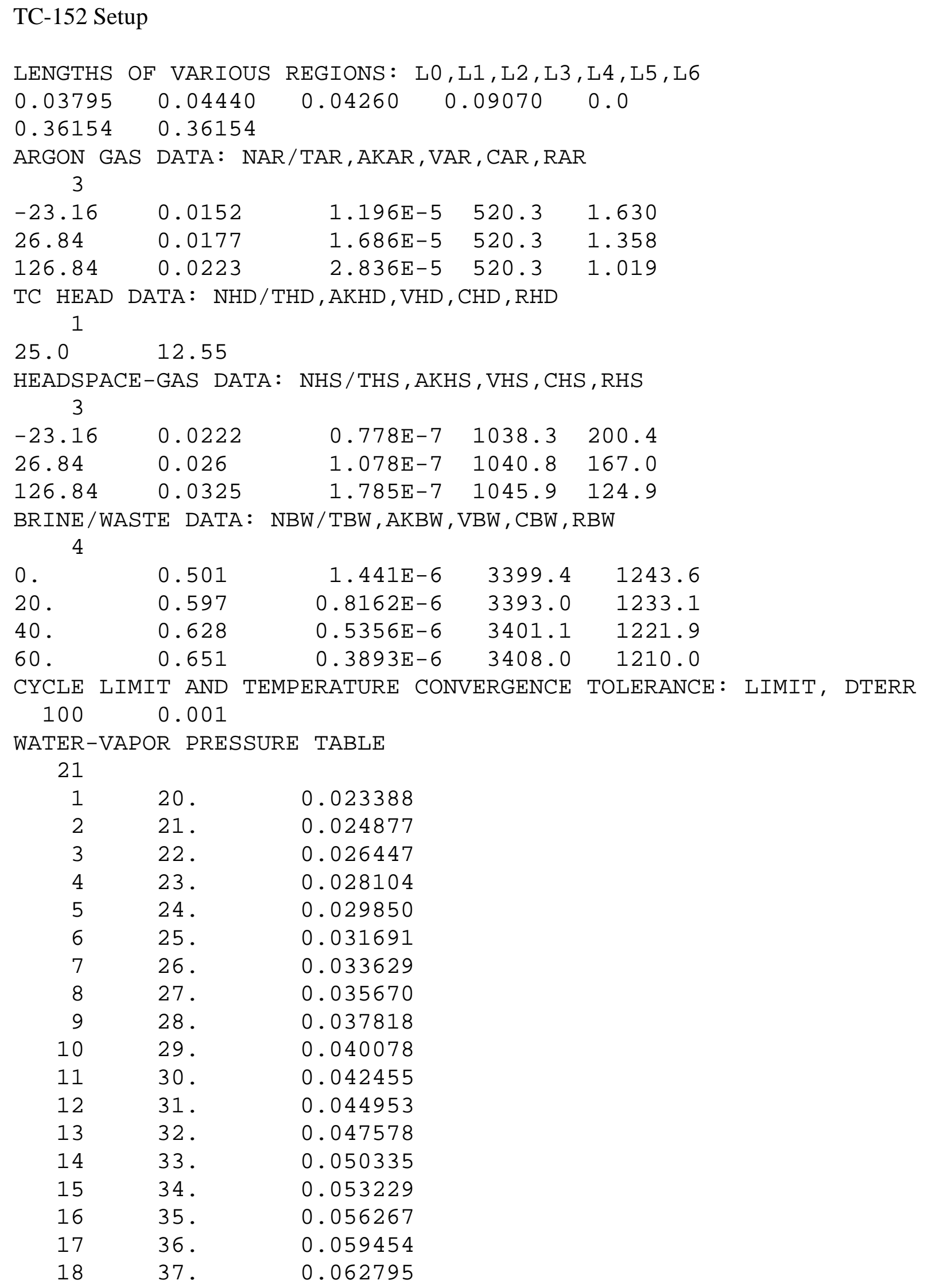




$$
\begin{array}{ccc}
19 & 38 . & 0.066298 \\
20 & 39 . & 0.069969 \\
21 & 40 . & 0.073814
\end{array}
$$

TEST-CONTAINER VOLUME \& LINEAR THERMAL-EXPANSION COEFFICIIENT 7272. $\quad 11.3 E-6$

GLOBAL EXCESS TEMP OF GB INTERIOR VS. THE GB RTD MEASUREMENT $\odot$.

NUMBER OF RTD \& PRESS XDUCR CALIBRATIONS: NCALT, NCALP 86

RTD CALIBRATION INFORMATION: [DATE:HR],PT NO , COMP, RESERR

\begin{tabular}{|c|c|c|c|}
\hline $11-03-95: ? ?$ & 1 & $\odot$. & -0.2 \\
\hline $07-15-96: ? ?$ & 776 & $\odot$. & -0.2 \\
\hline 01-13-97:?? & 5018 & $\odot$. & ○. \\
\hline 07- -9-97:?? & 9079 & 0. & 0.1 \\
\hline $11-21-97: ? ?$ & 12294 & $\odot$. & ○. \\
\hline $05-21-98: ? ?$ & 16560 & $\odot$. & $\odot$. \\
\hline $11-23-98: ? ?$ & 20938 & $\odot$. & ○. \\
\hline 05-24-99:?? & 25099 & $\odot$. & $\odot$ \\
\hline
\end{tabular}

PRESSURE XDUCER CALIBRATION INFO: [DATE:HR],PT NO, COMP, RESIDP

$\begin{array}{rrrr}06-06-96: ? ? & 1 & 0 . & \odot . \\ 12-05 \odot 96: ? ? & 4126 & \odot . & \odot . \\ 06-05-97: ? ? & 8271 & \odot . & \odot . \\ 12-02-97: ? ? & 12550 & \odot . & \odot . \\ 06-02-98: ? ? & 16841 & \odot . & \odot . \\ 12-07-98: ? ? & 21259 & \odot . & \odot .\end{array}$

OVERALL REFERENCE PT DATA: NPTP, PP, TRTDP, TGBP, TGBEXP

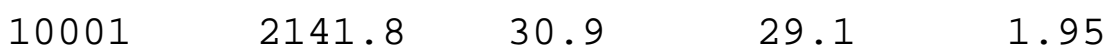

HS-GAS COMPRESSIBILITY TABLE DATA: NUM, VLO, VHI

$50 \quad 0.17 \quad 0.19$

BRINE MASS AND MOLECULAR WEIGHT: AMB[G],WTMOLB[G/GMOLE]

7894. 18.5

UNIVERSAL GAS CONSTANT: RU[PSI*ML/GMOLE/K], STD ATM PRESS[PSIA] $1205.95 \quad 14.696$

SOL GAS INFO: VOL FRACT[VFRG], MOL WT[WTMOLG], CONSTANTS A, B, C

$\odot . \quad-67.3877 \quad 86.3213 \quad 24.7981$

SAMPLE VOLUME: VSAMPL

3.05

NUMBER OF GAS-SAMPLE DATA POINTS: NSAMPL

4

GAS-SAMPLE DATA: [ID], NPTS( I ) ,PS1, PS2, TRTD, TGB, TGBEX

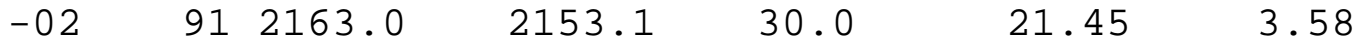

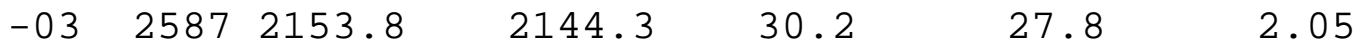

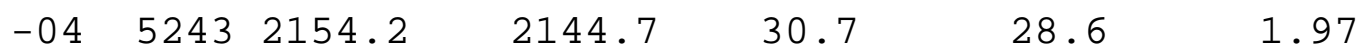

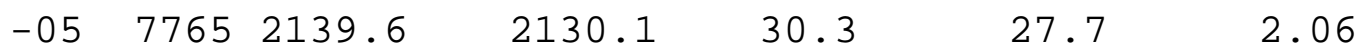

PXD BACKFILL INF0: [N0.,PT N0.],PC1,PC2, TC1, TC2, TGBC, TGBEXC

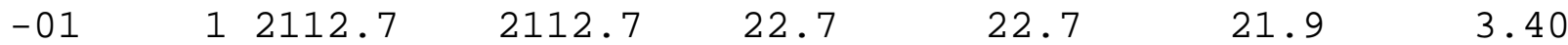




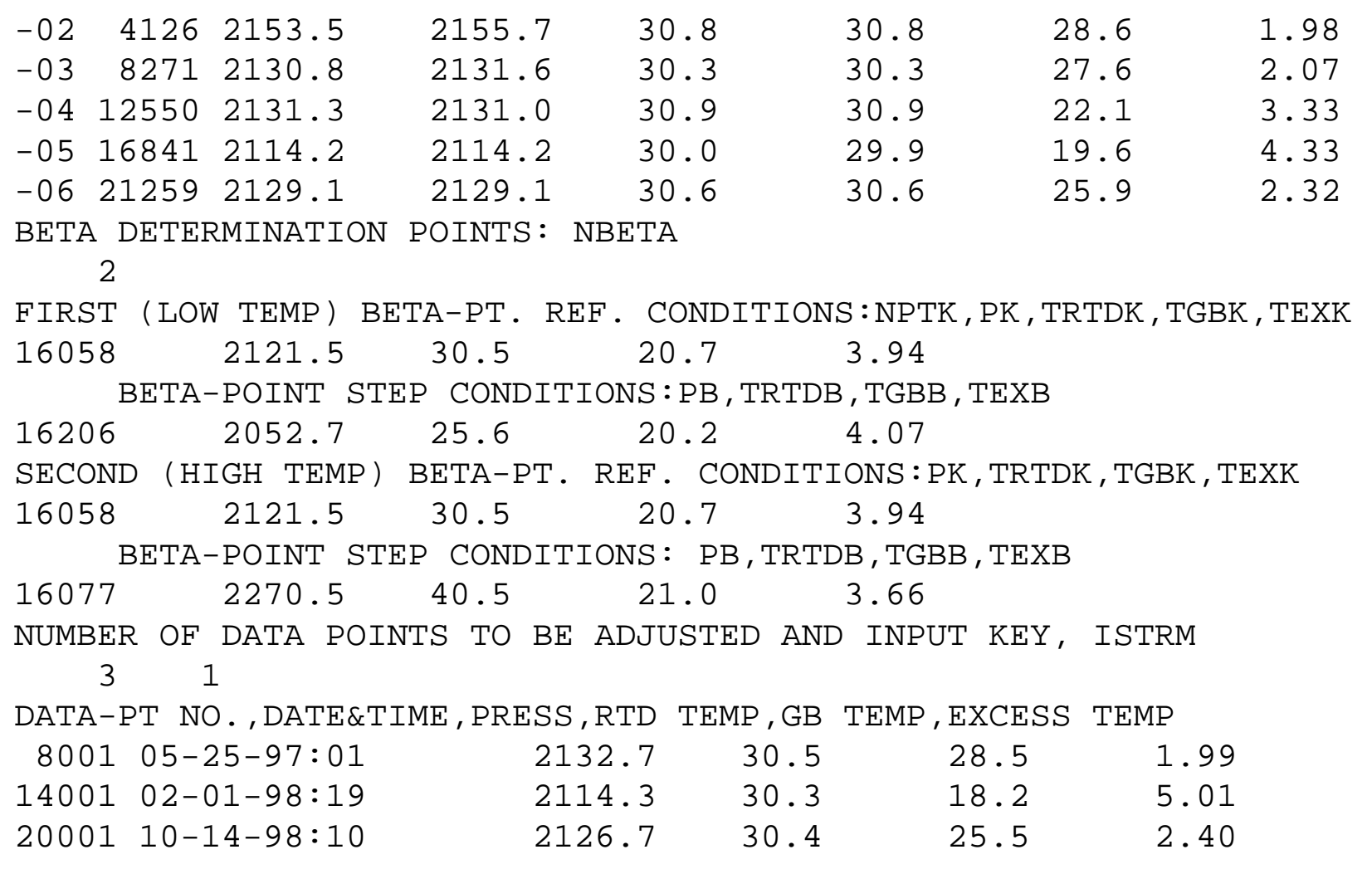




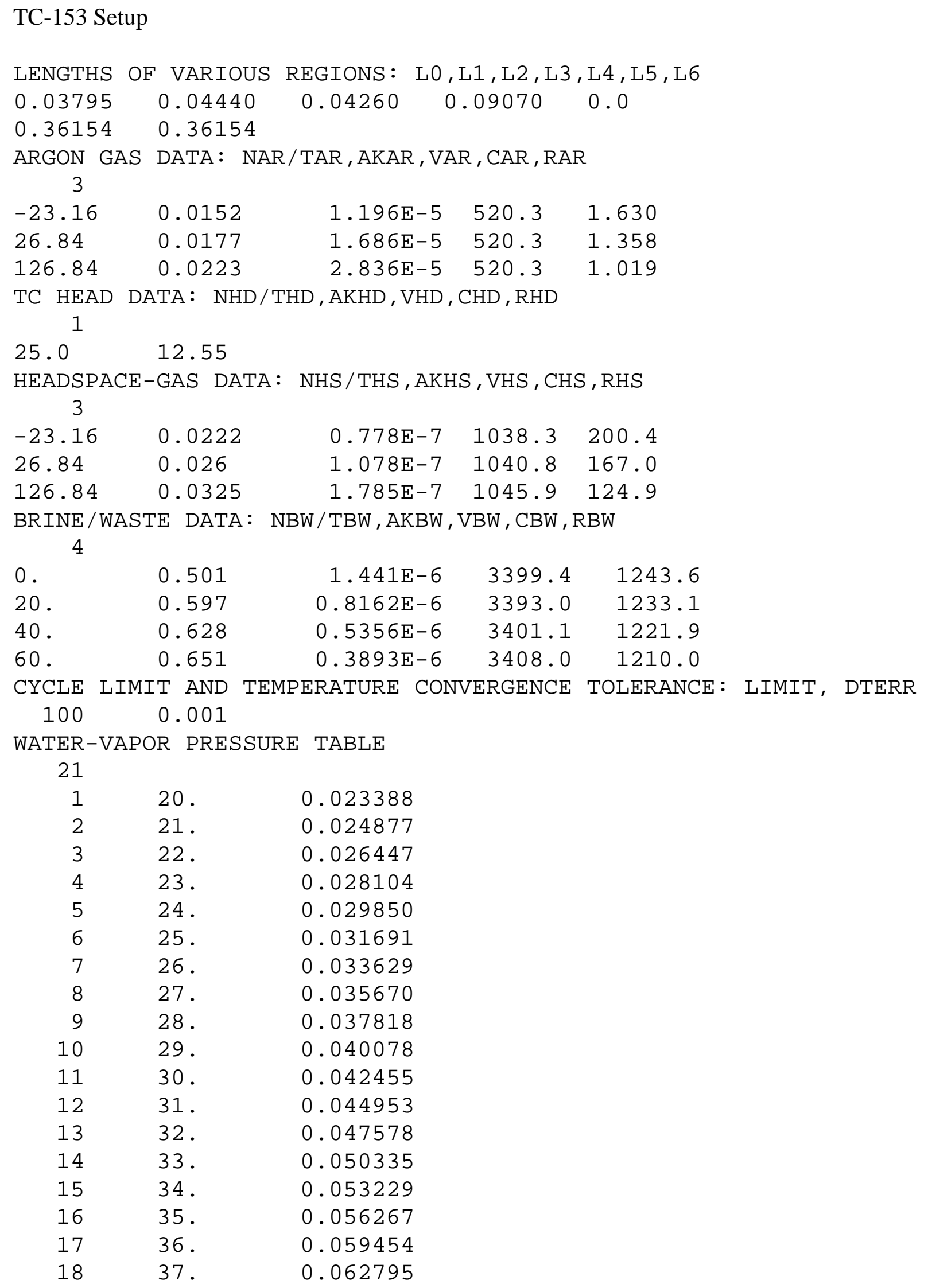




$\begin{array}{lll}19 & 38 . & 0.066298 \\ 20 & 39 . & 0.069969 \\ 21 & 40 . & 0.073814\end{array}$

TEST-CONTAINER VOLUME \& LINEAR THERMAL-EXPANSION COEFFICIENT 7272. $11.3 E-6$

GLOBAL EXCESS TEMP OF GB INTERIOR VS. THE GB RTD MEASUREMENT $\odot$.

NUMBER OF RTD \& PRESS XDUCR CALIBRATIONS: NCALT, NCALP 86

RTD CALIBRATION INFORMATION: [DATE:HR],PT NO , COMP, RESIDT

$\begin{array}{rrll}11-03-95: ? ? & 0 & 0 . & 0 . \\ 07-15-96: 14 & 597 & 0 . & 0 . \\ 01-13-97: ? ? & 4878 & 0 . & 0 . \\ 07-09-97: ? ? & 8976 & 0 . & 0 . \\ 11-21-97: ? ? & 12214 & -0.1 & 0 . \\ 05-21-98: ? ? & 16539 & 0 . & 0 . \\ 11-23-98: ? ? & 20893 & 0 . & 0 . \\ 05-24-99: ? ? & 25058 & 0 . & 0.1\end{array}$

PRESSURE XDUCER CALIBRATION INFO: [DATE:HR],PT NO., COMP, RESIDP

$\begin{array}{rrrr}06-13-96: ? ? & \odot & 0 . & 0 . \\ 12-05-96: ? ? & 3982 & \odot . & \odot . \\ 06-05-97: ? ? & 8164 & \odot . & \odot . \\ 12-02-97: ? ? & 12477 & \odot . & \odot . \\ 06-02-98: ? ? & 16881 & \odot . & \odot . \\ 12-07-98: ? ? & 21220 & \odot . & \odot .\end{array}$

OVERALL REFERENCE PT DATA: NPTP, PP, TRTDP, TGBP, TGBEXP

$\begin{array}{lllll}10001 & 2154.2 & 31.0 & 29.3 & 1.94\end{array}$

HS-GAS COMPRESSIBILITY TABLE DATA: NUM, VLO, VHI

$50 \quad 0.15 \quad 0.19$

BRINE MASS AND MOLECULAR WT: AMB [G], WTMOLB[G/GMOLE]

7868. $\quad 18.5$

UNIVERSAL GAS CONSTANT:RU [PSI*ML/GMOLE/K],ATM PRESS [PSIA]

$1205.95 \quad 14.696$

SOL GAS INFO: VOL FRACT[VFRG], MOL WT[WTMOLG], CONSTANTS A, B, C

$\odot . \quad-67.3877 \quad 86.3213 \quad 24.7981$

SAMPLE VOLUME: VSAMPL

3.05

NUMBER OF GAS-SAMPLE DATA POINTS: NSAMPL

4

GAS-SAMPLE DATA: [ID] , NPTS( I ), PS1, PS2, TRTD, TGB, TGBEX

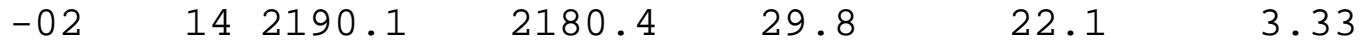

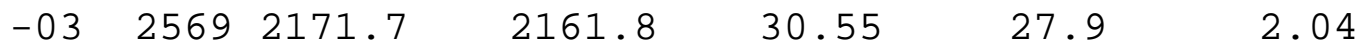

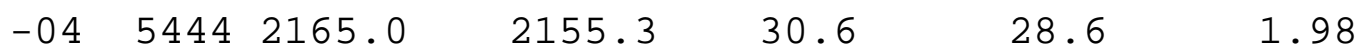

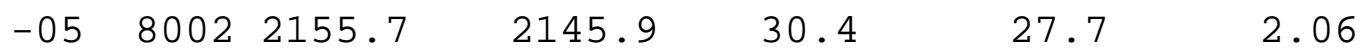

PXD BACKFILL INF0: [N0.,PT. NO.],PC1, PC2, TC1, TC2, TGBC, TGBEXC

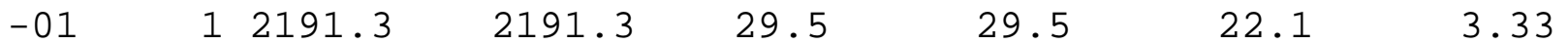




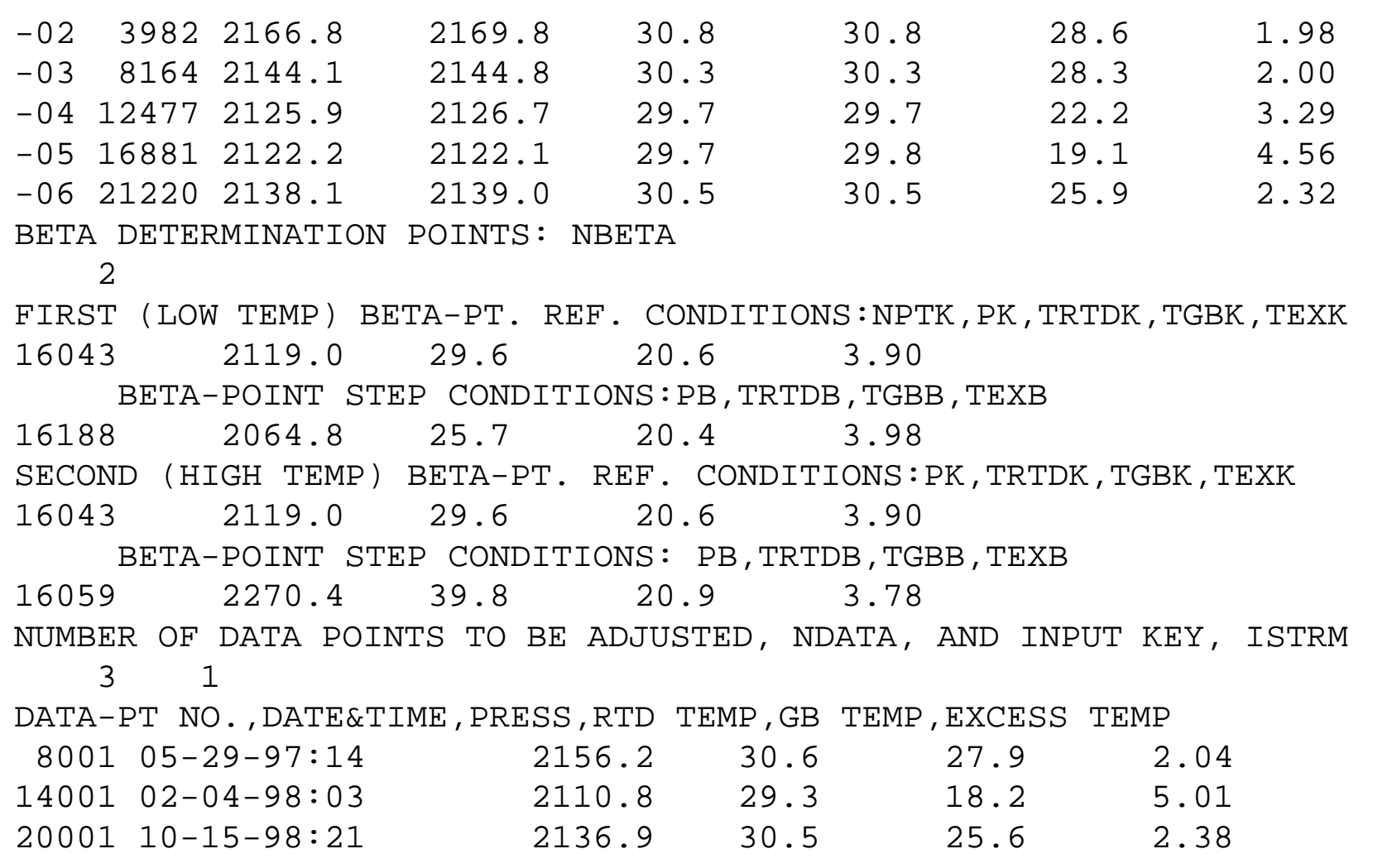




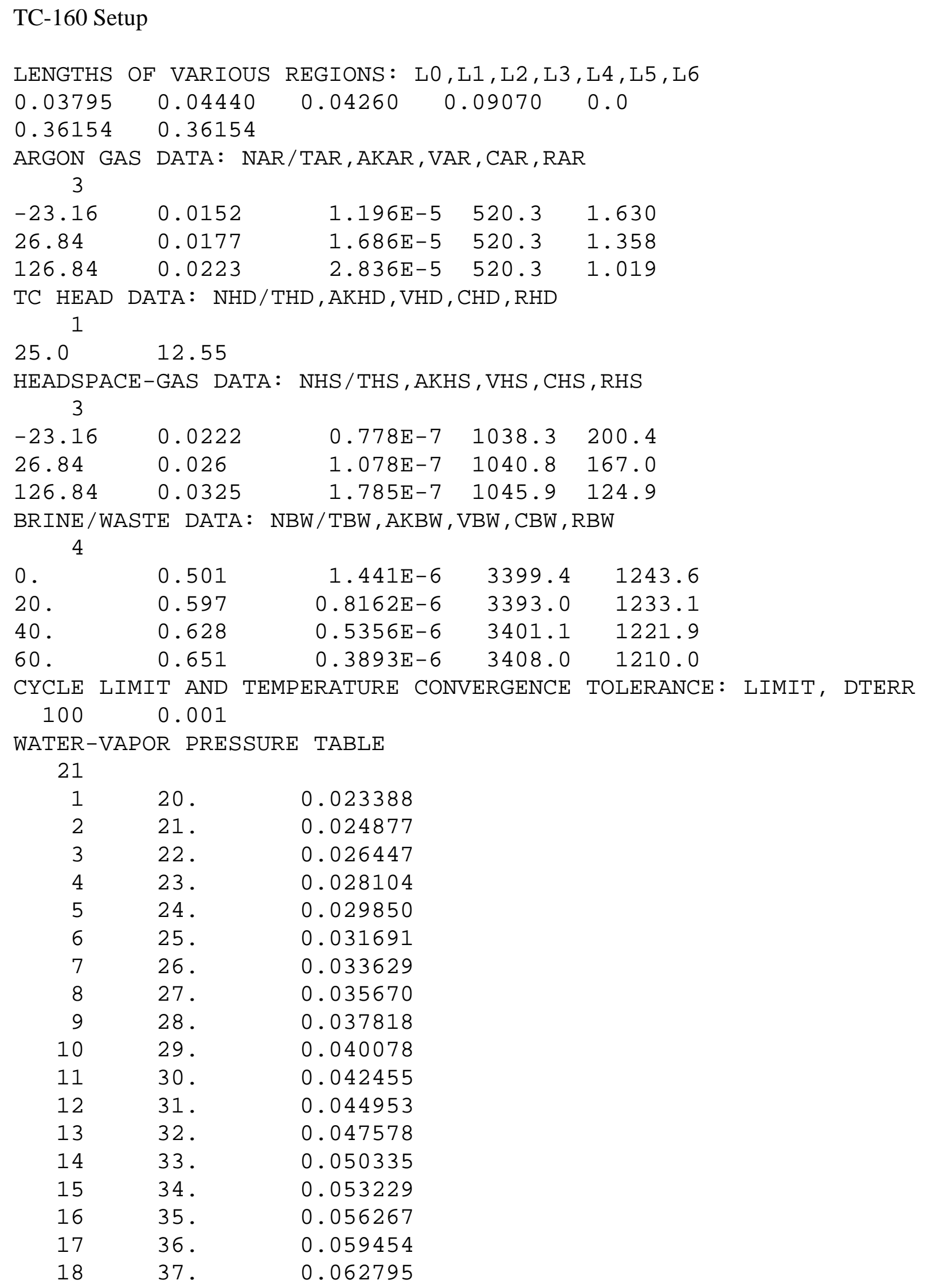




$\begin{array}{lll}19 & 38 . & 0.066298 \\ 20 & 39 . & 0.069969 \\ 21 & 40 . & 0.073814\end{array}$

TEST-CONTAINER VOLUME \& LINEAR THERMAL-EXPANSION COEFFICIIENT 7272. $11.3 E-6$

GLOBAL EXCESS TEMP OF GB INTERIOR VS. THE GB RTD MEASUREMENT $\odot$.

NUMBER OF RTD \& PXD CALIBRATIONS: NCALT, NCALP

86

RTD CALIBRATION INFORMATION: [DATE:HR],PT NO , COMP, RESIDT

$\begin{array}{lrll}11-03-95: ? ? & 1 & \odot . & \odot . \\ 07-15-96: ? ? & 2306 & \odot . & \odot . \\ 01-13-97: ? ? & 6544 & \odot . & -0.1 \\ 07-09-97: ? ? & 10609 & \odot . & -0.1 \\ 11-21-97: ? ? & 13823 & -0.3 & \odot . \\ 05-21-98: ? ? & 18111 & \odot . & -0.1 \\ 11-23-98: ? ? & 22489 & \odot . & \odot . \\ 05-24-99: ? ? & 26678 & \odot . & \odot .\end{array}$

PRESSURE XDUCER CALIBRATION: [DATE:HR], PT NO, COMP, RESIDP

$\begin{array}{rrll}\odot 8-13-96: 10 & 2957 & \odot . & \odot . \\ \odot 2-21-97: ? ? & 7428 & \odot . & \odot . \\ \odot 8-21-97: ? ? & 11632 & \odot . & \odot . \\ \odot 2-20-98: ? ? & 15981 & \odot . & \odot . \\ \odot 8-19-98: ? ? & 20240 & \odot . & \odot . \\ \odot 2-17-99: ? ? & 24409 & \odot . & \odot .\end{array}$

OVERALL REFERENCE PT DATA: NPTP, PP, TRTDP, TGBP, TGBEXP

$\begin{array}{lllll}10001 & 2128.0 & 31.2 & 28.6 & 1.98\end{array}$

HS-GAS COMPRESSIBILITY TABLE DATA: NUM, VLO, VHI

$50 \quad 0.17 \quad 0.19$

BRINE MASS \& MOLECULAR WEIGHT:AMB[G], WTMOLB[G/GMOLE]

6678. $\quad 18.5$

UNIVERSAL GAS CONSTANT: RU[PSI*ML/GMOLE/K],ATM PRESS[PSIA]

$1205.95 \quad 14.696$

SOL-GAS INFO: VOL FRACT (VFRG), MOL WT(WTMOLG), CONSTANTS A, B, C

$\odot . \quad-67.3877 \quad 86.3213 \quad 24.7981$

SAMPLE VOLUME: VSAMPL

3.05

NUMBER OF GAS-SAMPLE DATA POINTS: NSAMPL

4

GAS-SAMPLE DATA: [ ID ], NPTS( I ), PS1, PS2, TRTD, TGB, TGBEX

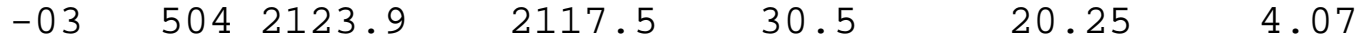

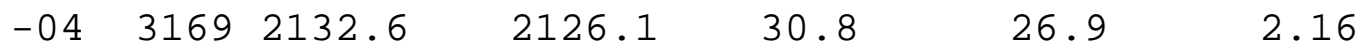

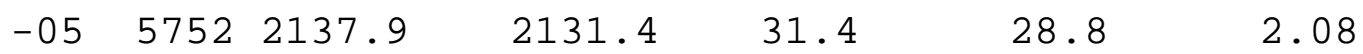

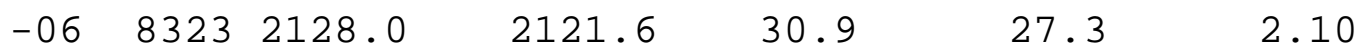

PXD BACKFILL INF0: [N0,PT N0], PC1, PC2, TC1, TC2, TGBC, TGBEXC

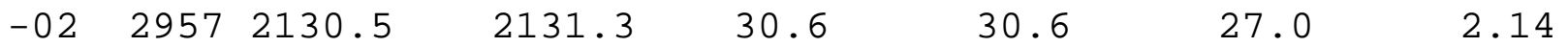




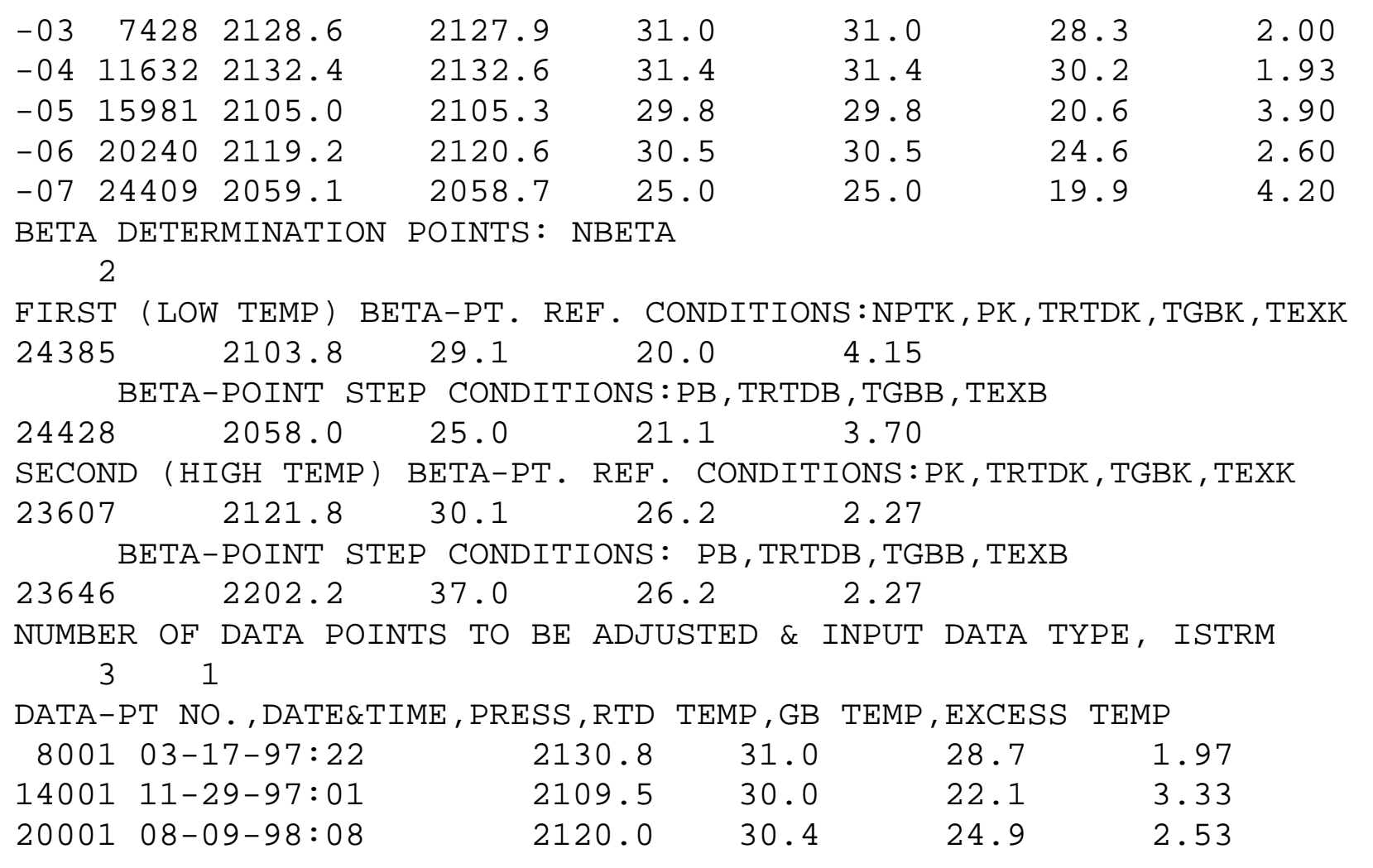




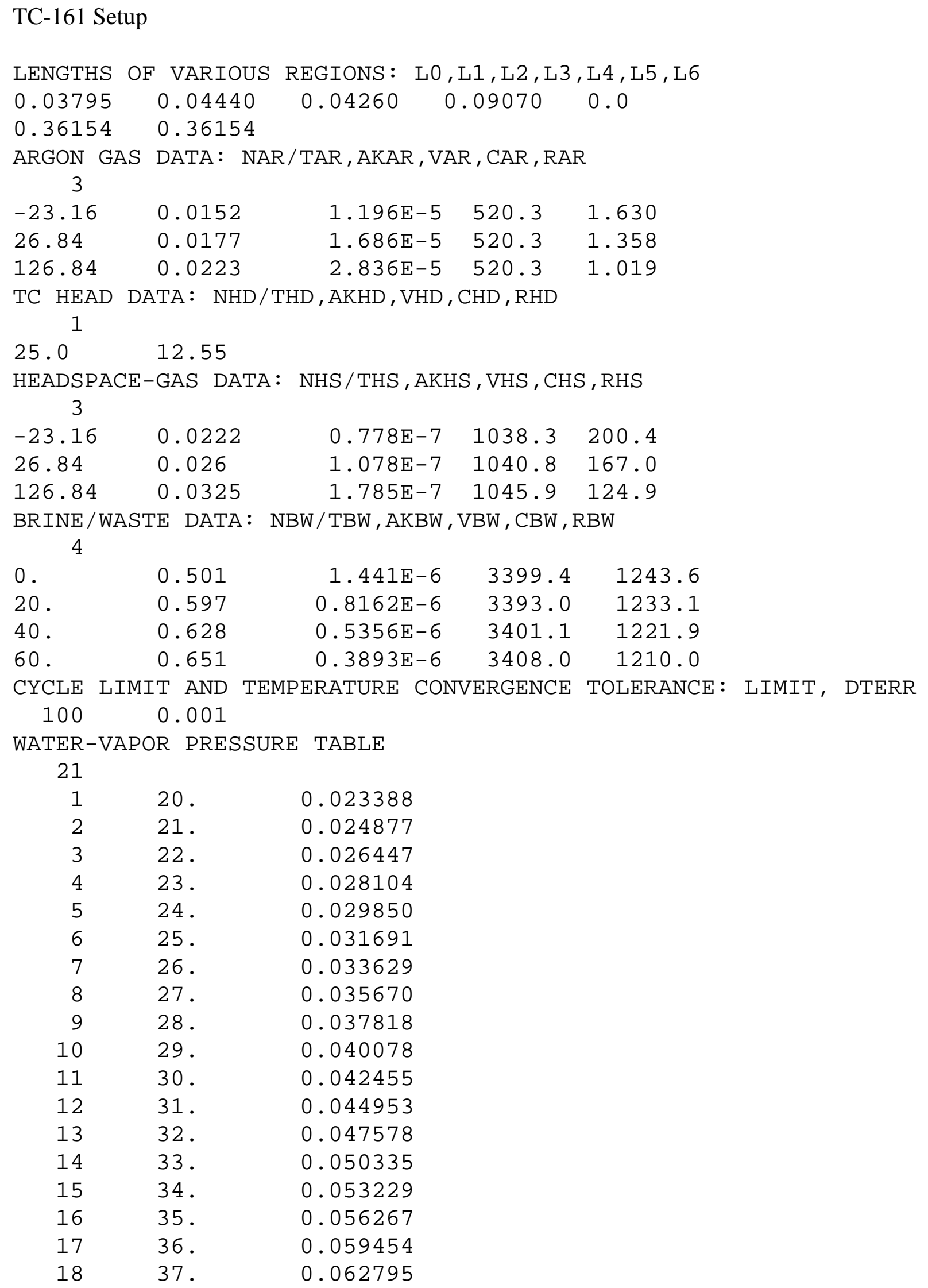




$\begin{array}{lll}19 & 38 . & 0.066298 \\ 20 & 39 . & 0.069969 \\ 21 & 40 . & 0.073814\end{array}$

TEST-CONTAINER VOLUME \& LINEAR THERMAL-EXPANSION COEFFICIIENT 7272. $11.3 E-6$

GLOBAL EXCESS TEMP OF GB INTERIOR VS. THE GB RTD MEASUREMENT $\odot$.

NUMBER OF RTD \& PXD CALIBRATIONS: NCALT, NCALP

86

RTD CALIBRATION INFORMATION: [DATE\&HR],PT NO . COMP, RESIDT

\begin{tabular}{|c|c|c|c|}
\hline $02-12-96: ? ?$ & 1 & $\odot$. & $\odot$. \\
\hline $07-15-96: ? ?$ & 3323 & 0.5 & $\odot$. \\
\hline 01-13-97:?? & 7558 & $\Theta$. & $\odot$. \\
\hline ०7-09-97:?? & 11626 & $\Theta$. & 0.1 \\
\hline 11-21-97:?? & 14848 & 0.6 & 0.1 \\
\hline $05-21-98: ? ?$ & 19142 & -0.9 & $\odot$. \\
\hline $11-21-98: ? ?$ & 23524 & 0.4 & 0. \\
\hline $05-24-99: ? ?$ & 27711 & $\odot$. & -0.1 \\
\hline
\end{tabular}

PXD CALIBRATION INFO: [DATE\&HR], PT NO, COMP, RESIDP

०8-13-96:10 $3970 \quad 0$.

02-24-97:?? $8513 \quad 0 . \quad 0$.

०8-21-97:?? $12652 \quad 0 . \quad 0$.

02-20-98:?? $17002 \quad 0 . \quad 0$.

०8-19-98:?? $21268 \quad 0 . \quad 0$.

02-17-99:?? $25433 \quad 0$.

OVERALL REFERENCE PT DATA: NPTP, PP, TRTDP, TGBP, TGBEXP

$\begin{array}{lllll}10001 & 2098.1 & 31.2 & 28.6 & 1.98\end{array}$

HS-GAS COMPRESSIBILITY TABLE DATA: NUM, VLO, VHI

$50 \quad 0.17 \quad 0.19$

BRINE MASS \& MOLECULAR WEIGHT: AMB[G], WTMOLB[G/GMOLE]

6872. $\quad 18.5$

UNIVERSAL GAS CONSTANT: RU[PSI*ML/GMOLE/K],ATM PRESS[PSIA]

$1205.95 \quad 14.696$

SOL GAS INFO: VOL FRACT[VFRG], MOL WT (WTMOLG), CONSTANTS A, B, C

$\odot . \quad-67.3877 \quad 86.3213 \quad 24.7981$

SAMPLE VOLUME: VSAMPL

3.05

NUMBER OF GAS-SAMPLE DATA POINTS: NSAMPL

5

GAS-SAMPLE DATA: [ ID ], NPTS( I ), PS1, PS2, TRTDS, TGBS, TGBEXS

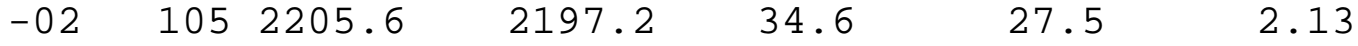

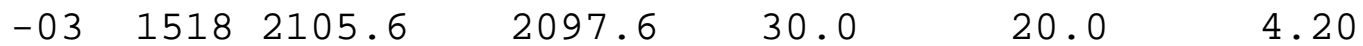

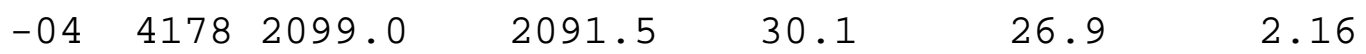

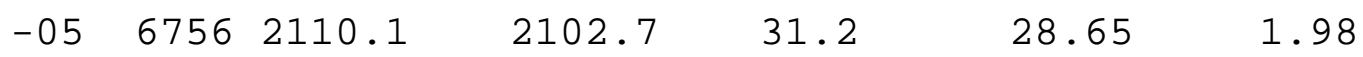

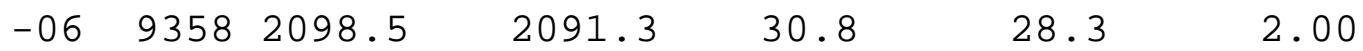

PXD BACKFILL INF0: [N0, PT NO], PC1, PC2, TC1, TC2, TGBC, TGBEXC 


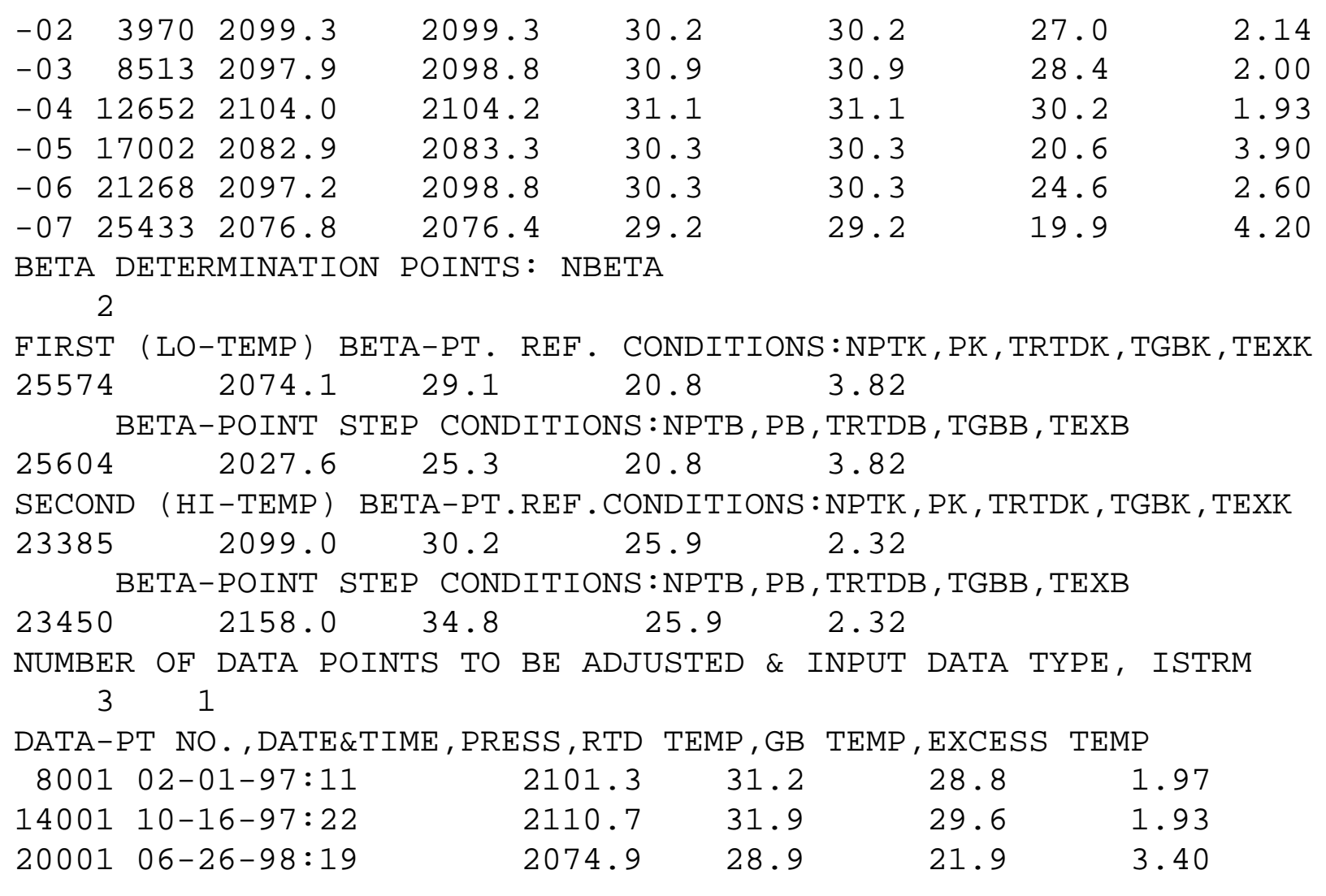




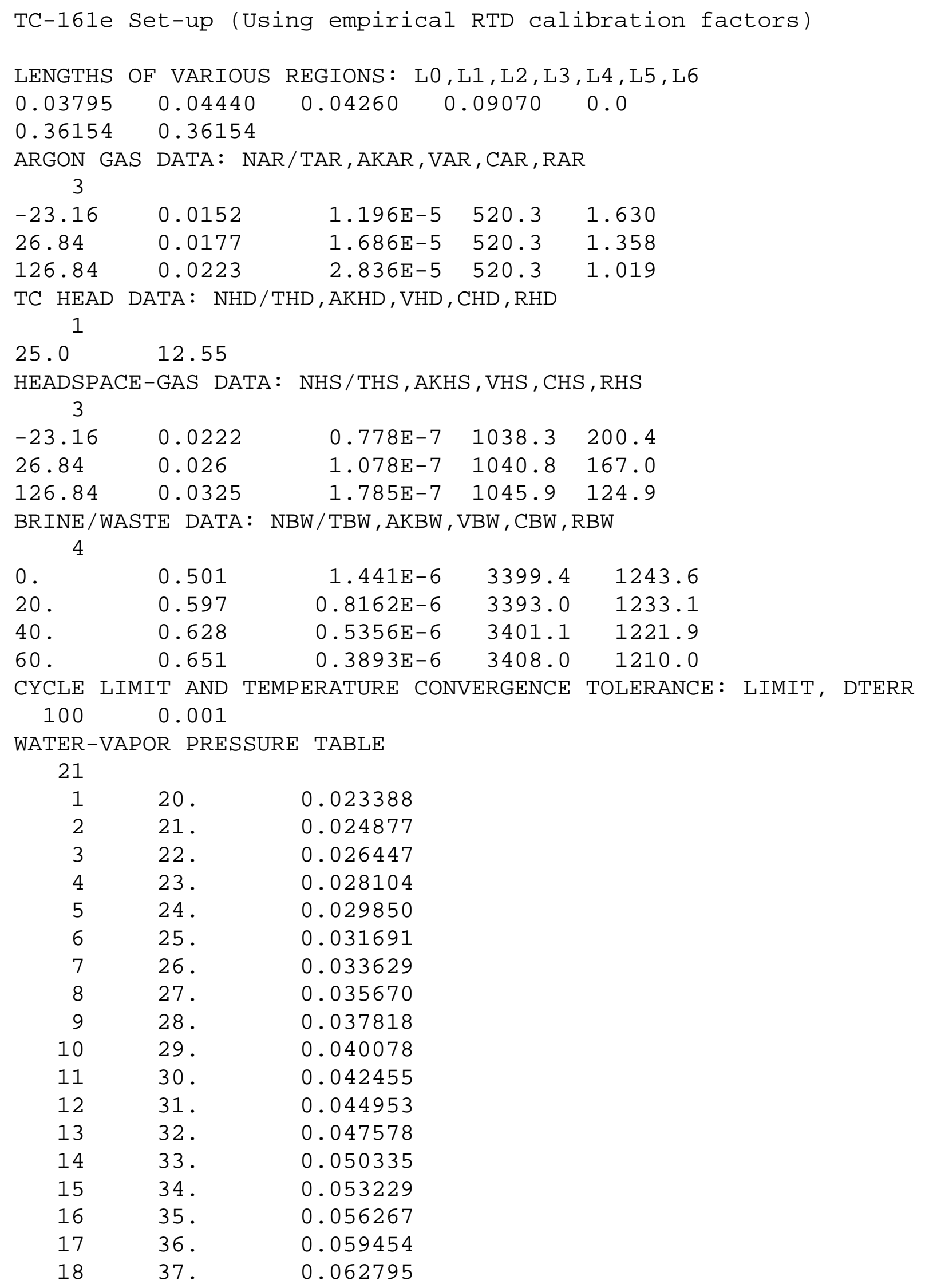




$\begin{array}{lll}19 & 38 . & 0.066298 \\ 20 & 39 . & 0.069969 \\ 21 & 40 . & 0.073814\end{array}$

TEST-CONTAINER VOLUME \& LINEAR THERMAL-EXPANSION COEFFICIIENT 7272. $11.3 E-6$

GLOBAL EXCESS TEMP OF GB INTERIOR VS. THE GB RTD MEASUREMENT $\odot$.

NUMBER OF RTD \& PXD CALIBRATIONS: NCALT, NCALP

86

RTD CALIBRATION INFORMATION: [DATE\&HR],PT NO . COMP, RESIDT

\begin{tabular}{|c|c|c|c|}
\hline $02-12-96: ? ?$ & 1 & $\odot$. & $\odot$. \\
\hline $07-15-96: ? ?$ & 3323 & 0.8 & $\odot$. \\
\hline 01-13-97:?? & 7558 & 0.3 & -0.1 \\
\hline ०7- -9-97:?? & 11626 & -0.35 & 0.45 \\
\hline $11-21-97: ? ?$ & 14848 & 0.6 & ○. \\
\hline 05-21-98:?? & 19142 & -0.7 & 0.7 \\
\hline $11-21-98: ? ?$ & 23524 & 0.6 & -0.1 \\
\hline ०5-24-99:?? & 27711 & 0.1 & 0 \\
\hline
\end{tabular}

PXD CALIBRATION INFO: [DATE\&HR], PT NO, COMP, RESIDP

०8-13-96:10 $3970 \quad 0$.

02-24-97:?? $8513 \quad 0 . \quad 0$.

०8-21-97:?? $12652 \quad 0 . \quad 0$.

02-20-98:?? $17002 \quad 0 . \quad 0$.

०8-19-98:?? $21268 \quad 0 . \quad 0$.

02-17-99:?? $25433 \quad 0 . \quad 0$.

OVERALL REFERENCE PT DATA: NPTP, PP, TRTDP, TGBP, TGBEXP

$\begin{array}{lllll}10001 & 2098.1 & 31.2 & 28.6 & 1.98\end{array}$

HS-GAS COMPRESSIBILITY TABLE DATA: NUM, VLO, VHI

$50 \quad 0.17 \quad 0.19$

BRINE MASS \& MOLECULAR WEIGHT: AMB[G], WTMOLB[G/GMOLE]

6872. $\quad 18.5$

UNIVERSAL GAS CONSTANT: RU[PSI*ML/GMOLE/K],ATM PRESS[PSIA]

$1205.95 \quad 14.696$

SOL GAS INFO: VOL FRACT[VFRG], MOL WT (WTMOLG), CONSTANTS A, B, C

$\odot . \quad-67.3877 \quad 86.3213 \quad 24.7981$

SAMPLE VOLUME: VSAMPL

3.05

NUMBER OF GAS-SAMPLE DATA POINTS: NSAMPL

5

GAS-SAMPLE DATA: [ ID ], NPTS( I ), PS1, PS2, TRTDS, TGBS, TGBEXS

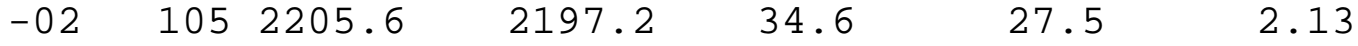

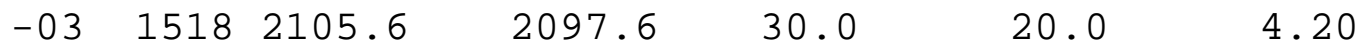

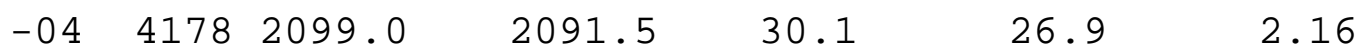

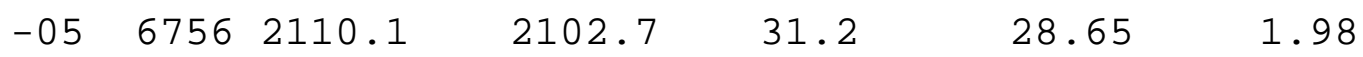

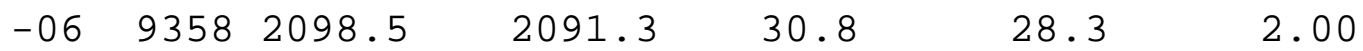

PXD BACKFILL INF0: [N0, PT NO], PC1, PC2, TC1, TC2, TGBC, TGBEXC 


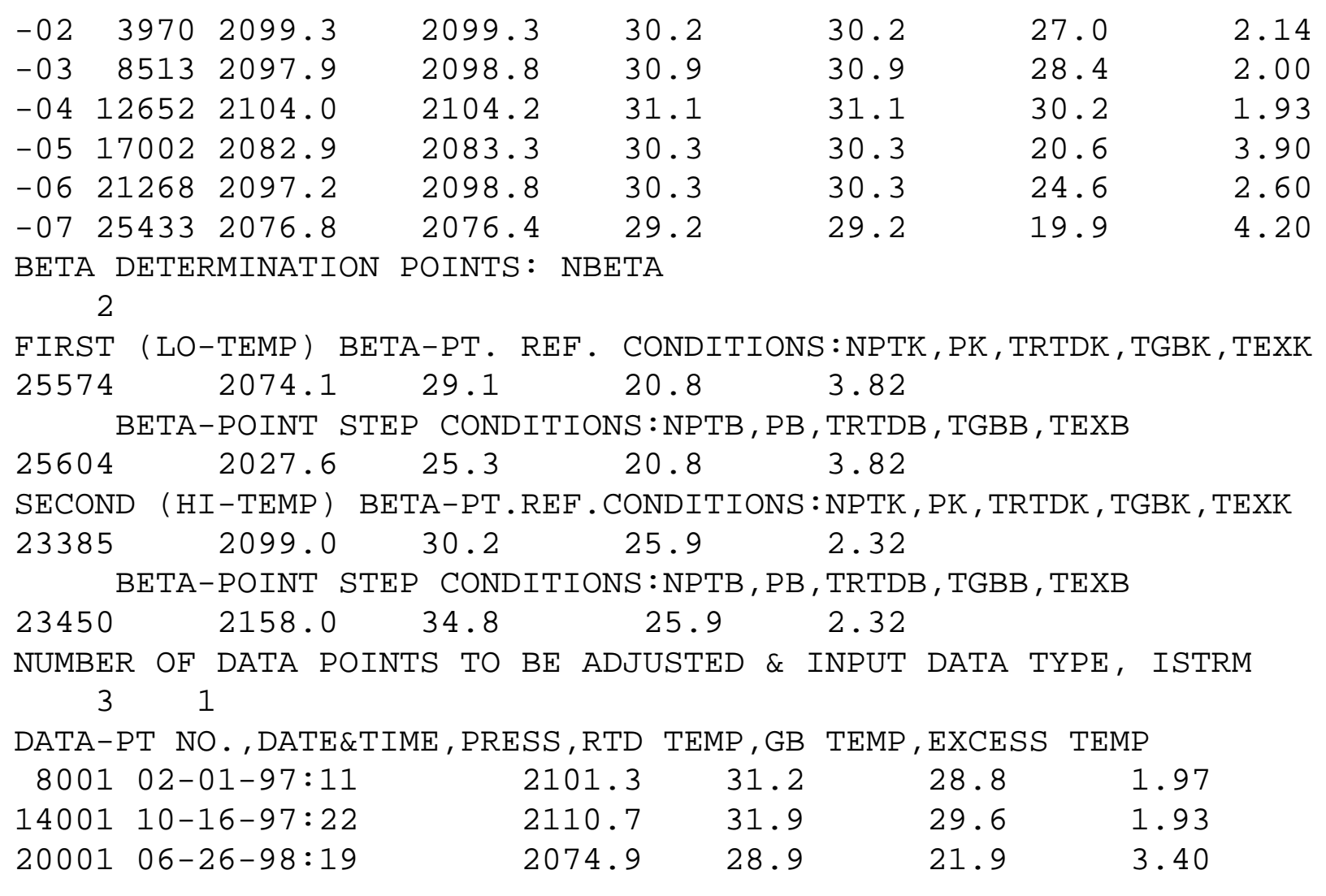




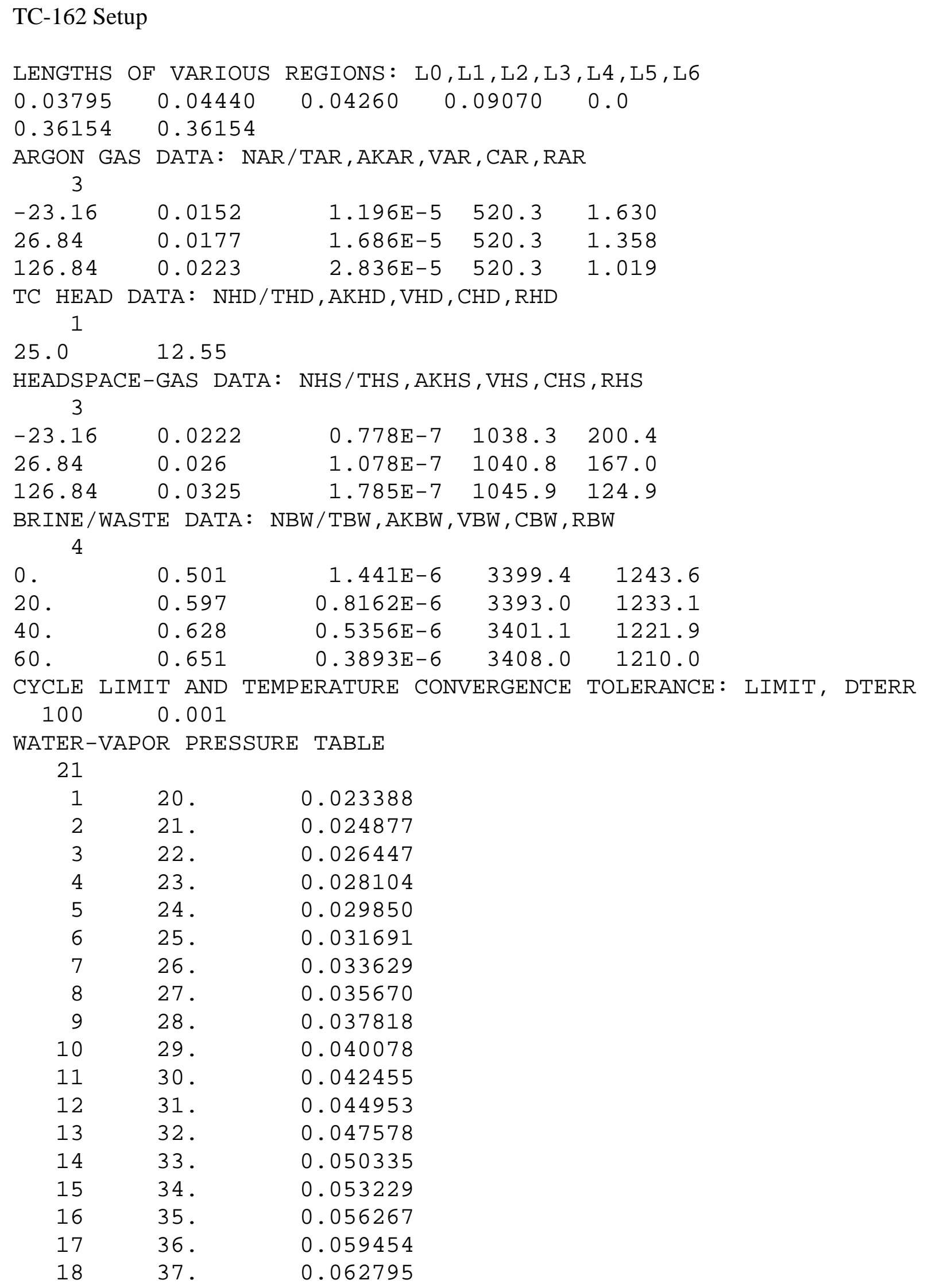




$\begin{array}{lll}19 & 38 . & 0.066298 \\ 20 & 39 . & 0.069969 \\ 21 & 40 . & 0.073814\end{array}$

TEST-CONTAINER VOLUME \& LINEAR THERMAL-EXPANSION COEFFICIIENT 7272. $11.3 E-6$

GLOBAL EXCESS TEMP OF GB INTERIOR VS. THE GB RTD MEASUREMENT $\odot$.

NUMBER OF RTD \& PXD CALIBRATIONS: NCALT, NCALP

$8 \quad 7$

RTD CALIBRATION INFORMATION: [DATE:HR],PT NO , COMP, RESERR

\begin{tabular}{|c|c|c|c|}
\hline $11-03-95: ? ?$ & 1 & $\odot$. & $\odot$. \\
\hline $07-15-96: ? ?$ & 3030 & $\odot$. & $\odot$. \\
\hline $01-13-97: ? ?$ & 7260 & $\odot$. & $\odot$. \\
\hline $07-09-97: ? ?$ & 11320 & $\odot$. & $\Theta$. \\
\hline $11-21-97: ? ?$ & 14528 & $\odot$. & $\odot$. \\
\hline 05-21-98:?? & 18804 & $\odot$. & -0.1 \\
\hline $11-23-98: ? ?$ & 23162 & $\odot$. & $\odot$. \\
\hline ๑5-24-99:?? & 27331 & $\odot$. & $\odot$. \\
\hline
\end{tabular}

PXD CALIBRATIONS [DATE:HR], PT NO, COMP, RESIDP

$\begin{array}{lrll}\odot 3-01-96: 11 & 18 & \odot . & \odot . \\ \odot 8-13-96: 11 & 3677 & -\odot .75 & \odot . \\ \odot 2-21-97: ? ? & 8143 & \odot . & \odot . \\ \odot 8-21-97: ? ? & 12339 & \odot . & \odot . \\ \odot 2-2 \odot-98: ? ? & 16674 & \odot . & \odot . \\ \odot 8-19-98: ? ? & 20925 & \odot . & \odot . \\ \odot 2-17-99: ? ? & 25071 & \odot . & \odot .\end{array}$

OVERALL REFERENCE PT DATA: NPTP, PP, TRTDP, TGBP, TGBEXP

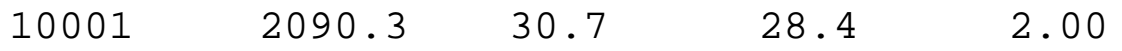

HS-GAS COMPRESSIBILITY TABLE DATA: NUM, VLO, VHI

$50 \quad 0.17 \quad 0.19$

BRINE MASS AND MOLECULAR WT: AMB [G],WTMOLB [G/GMOLE]

6891. 18.5

UNIVERSAL GAS CONSTANT: RU [PSI*ML/GMOLE/K],ATM PRESS [PSIA] $1205.95 \quad 14.696$

SOL GAS INFO: VOL FRACT[VFRG], MOL WT[WTMOLG], CONSTANTS A, B, C $\odot . \quad-67.3877 \quad 86.3213 \quad 24.7981$

SAMPLE VOLUME: VSAMPL

3.05

NUMBER OF GAS-SAMPLE DATA POINTS: NSAMPL

6

GAS-SAMPLE DATA: [ ID ], NPTS( I ) , PS1, PS2, TRTD, TGB, TGBEX

$\begin{array}{lrlllll}-03 & 20 & 2155.2 & 2147.4 & 30.1 & 27.2 & 2.12 \\ -04 & 107 & 2142.0 & 2134.8 & 29.9 & 26.15 & 2.27 \\ -05 & 1447 & 2107.3 & 2099.1 & 30.1 & 20.15 & 4.11 \\ -06 & 3982 & 2110.0 & 2102.4 & 30.7 & 27.25 & 2.12 \\ -07 & 6656 & 2113.4 & 2105.8 & 31.4 & 28.7 & 1.97\end{array}$




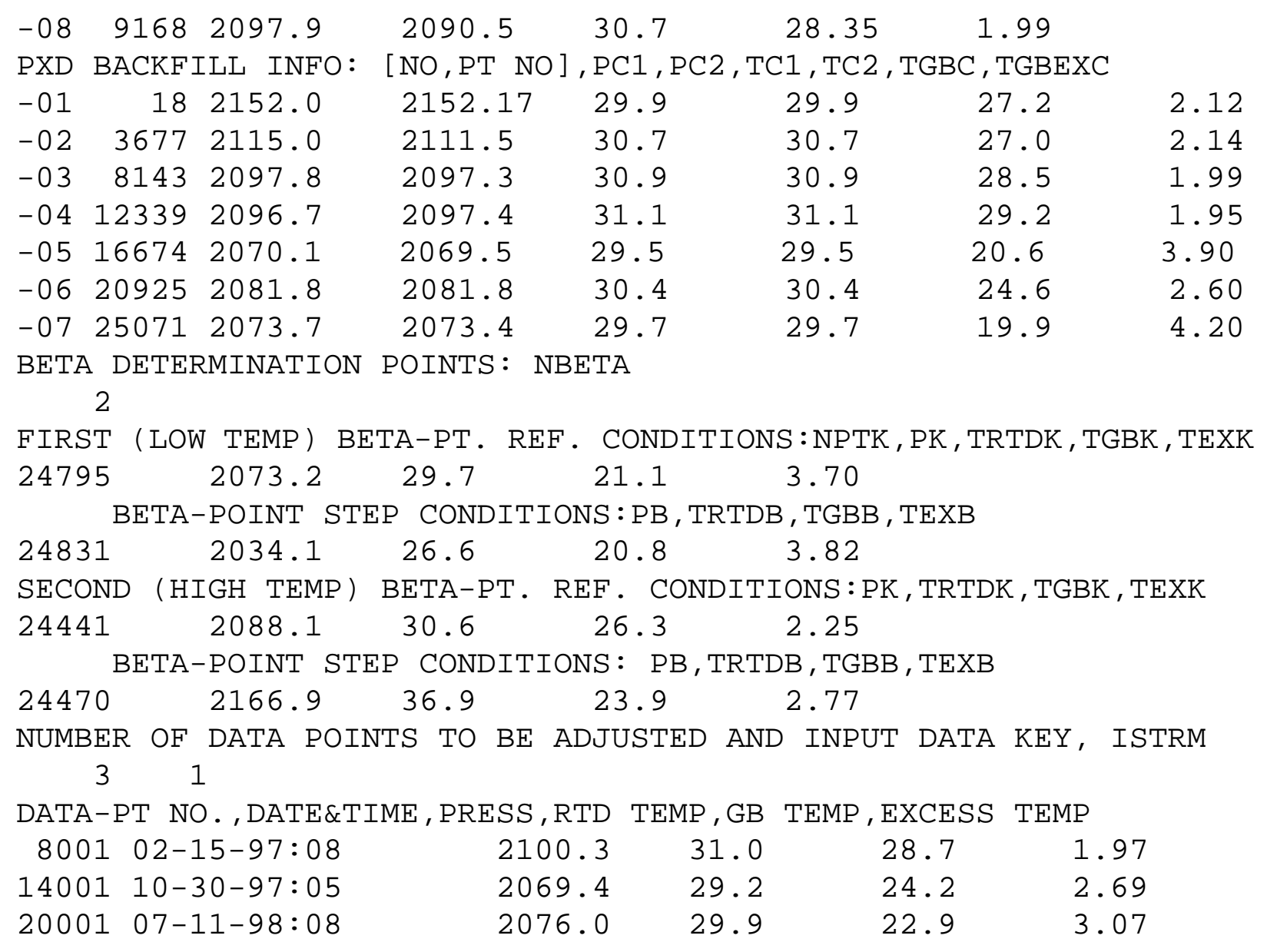




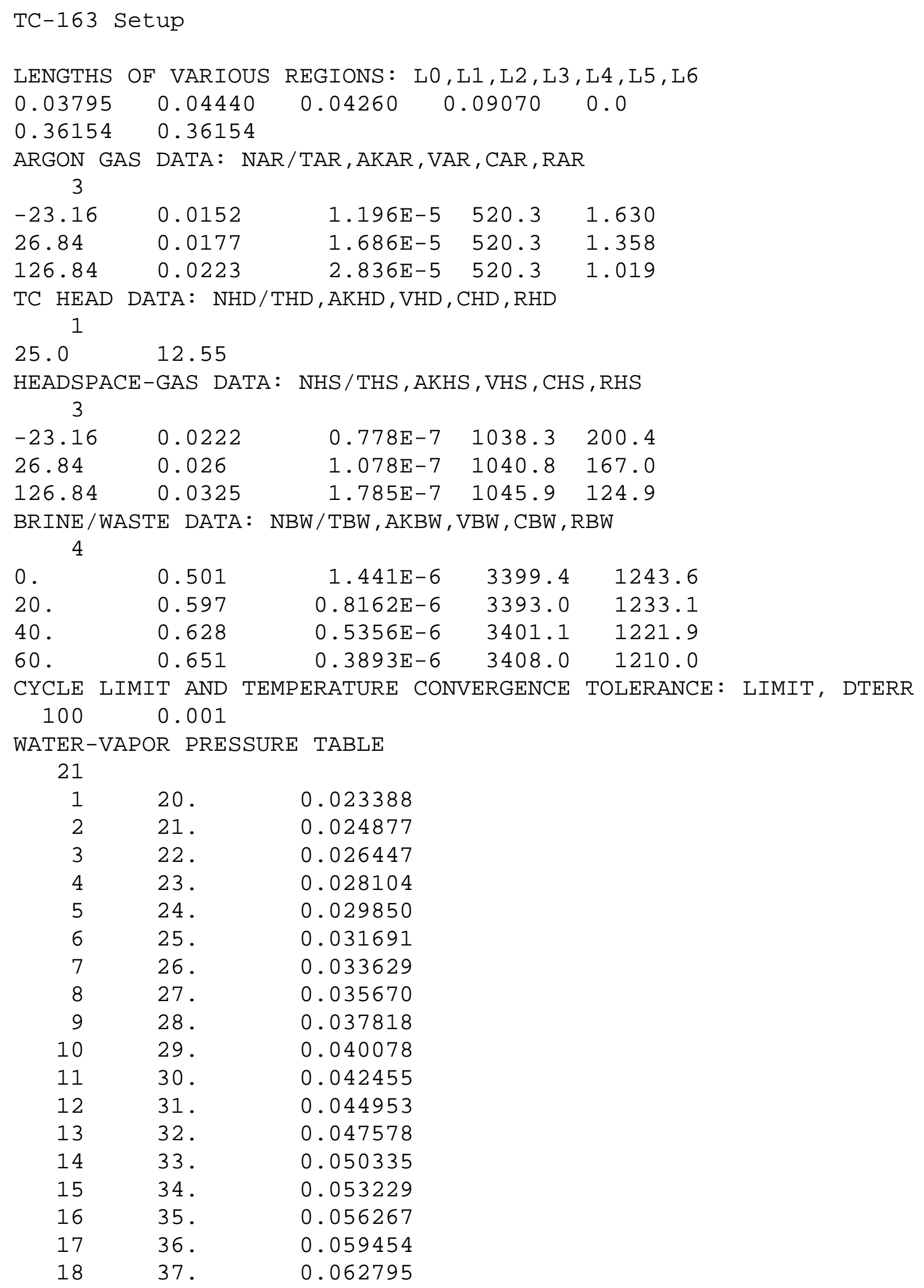




$\begin{array}{lll}19 & 38 . & 0.066298 \\ 20 & 39 . & 0.069969 \\ 21 & 40 . & 0.073814\end{array}$

TEST-CONTAINER VOLUME \& LINEAR THERMAL-EXPANSION COEFFICIIENT 7272. $11.3 E-6$

GLOBAL EXCESS TEMP OF GB INTERIOR VS. THE GB RTD MEASUREMENT $\odot$.

NUMBER OF RTD \& PXD CALIBRATIONS: NCALT, NCALP

86

RTD CALIBRATION INFORMATION: [DATE:HR],PT NO., COMP, RESERR

$\begin{array}{rrll}11-03-95: ? ? & 1 & \odot . & -0.1 \\ 07-15-96: ? ? & 2729 & \odot . & -0.1 \\ 01-13-97: ? ? & 6968 & \odot . & -0.1 \\ 07-09-97: ? ? & 11008 & \odot . & \odot . \\ 11-21-97: ? ? & 14220 & -0.1 & \odot . \\ 05-21-98: ? ? & 18497 & \odot . & -0.1 \\ 11-23-98: ? ? & 22857 & \odot . & \odot . \\ 05-24-99: ? ? & 27030 & \odot . & \odot .1\end{array}$

PRESSURE XDUCR CALIBRATIONS: [DATE:HR],PT NO, COMP, RESIDP

$\begin{array}{rrll}\text { 05-20-96:?? } & 1561 & 0 . & 0 . \\ 11-14-96: ? ? & 5581 & \odot . & 0 . \\ 05-14-97: ? ? & 9698 & \odot . & \odot . \\ 12-02-97: ? ? & 14473 & \odot . & 0 . \\ 06-01-98: ? ? & 18754 & \odot . & \odot . \\ 12-01-98: ? ? & 23041 & \odot . & 0 .\end{array}$

OVERALL REFERENCE PT DATA: NPTP, PP, TRTDP, TGBP, TGBEXP

$\begin{array}{lllll}10001 & 2173.0 & 31.0 & 28.5 & 1.99\end{array}$

HS-GAS COMPRESSIBILITY TABLE DATA: NUM, VLO, VHI

$$
50 \quad 0.17 \quad 0.19
$$

BRINE MASS \& MOLECULAR WT: AMB [G],WTMOLB [G/GMOLE]

7708. $\quad 18.5$

UNIVERSAL GAS CONSTANT: RU [PSI*ML/GMOLE/K], ATM PRESS [PSIA] $1205.95 \quad 14.696$

SOL GAS INFO: VOL FRACT [VFRG], MOL WT[WTMOLG], CONSTANTS A, B, C $\odot . \quad-67.3877 \quad 86.3213 \quad 24.7981$

SAMPLE VOLUME: VSAMPL

3.05

NUMBER OF GAS-SAMPLE DATA POINTS: NSAMPL

5

GAS-SAMPLE DATA: [ID], NPTS( I ), PS1, PS2, TRTD, TGB, TGBEX

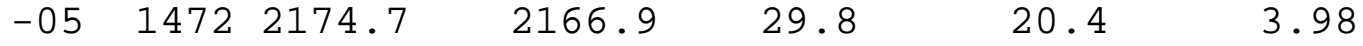

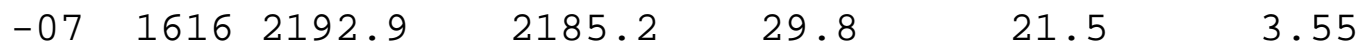

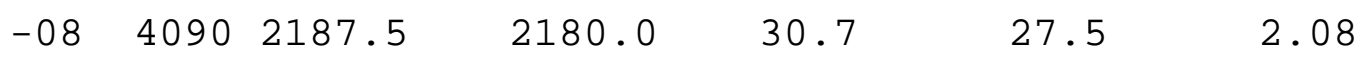

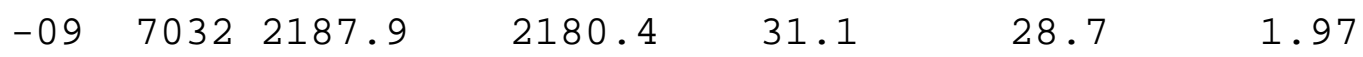

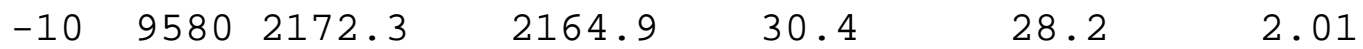

PXD BACKFILL INF0: [N0,PT NO], PC1, PC2, TC1, TC2, TGBC, TGBEXC 


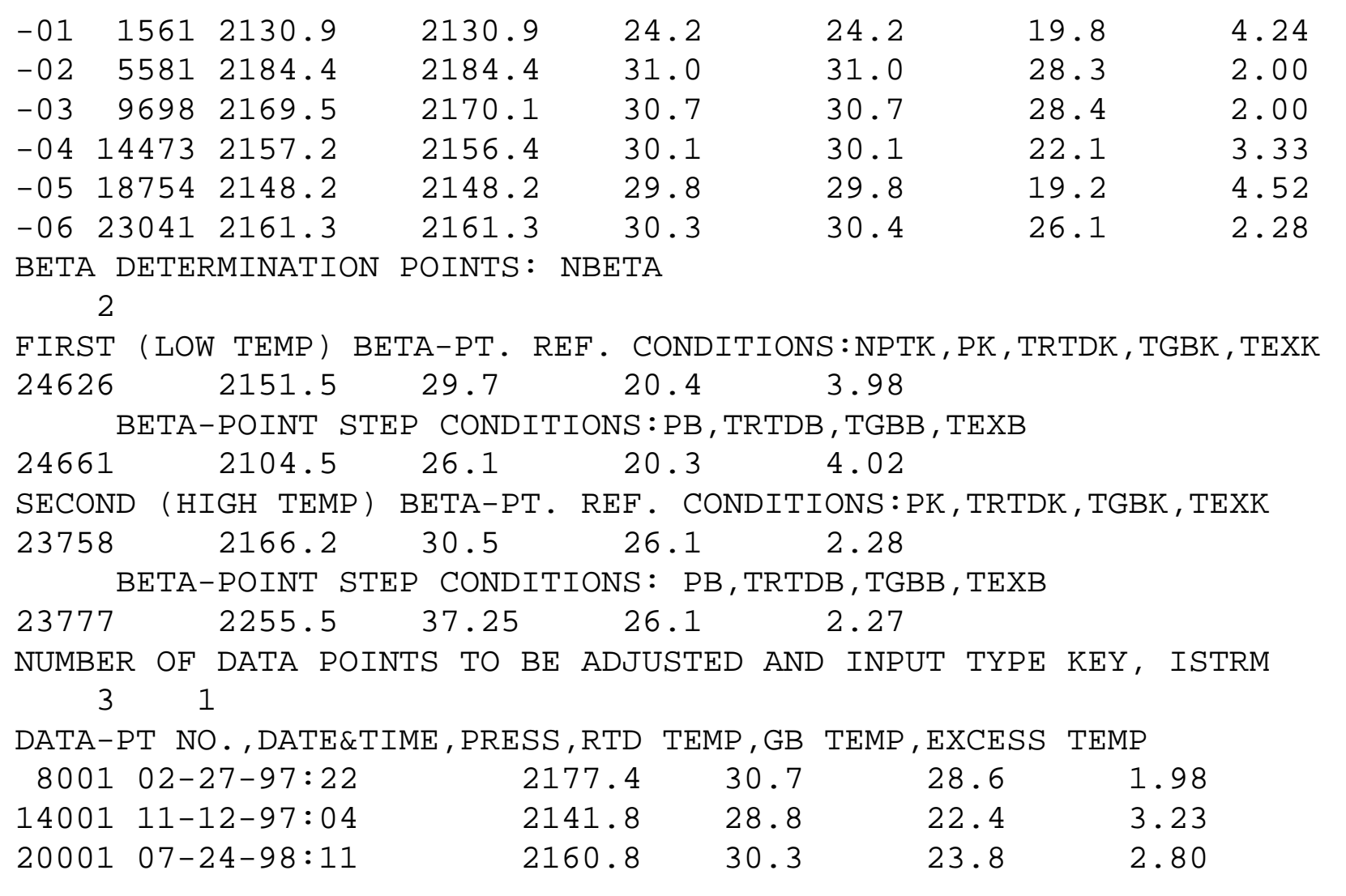




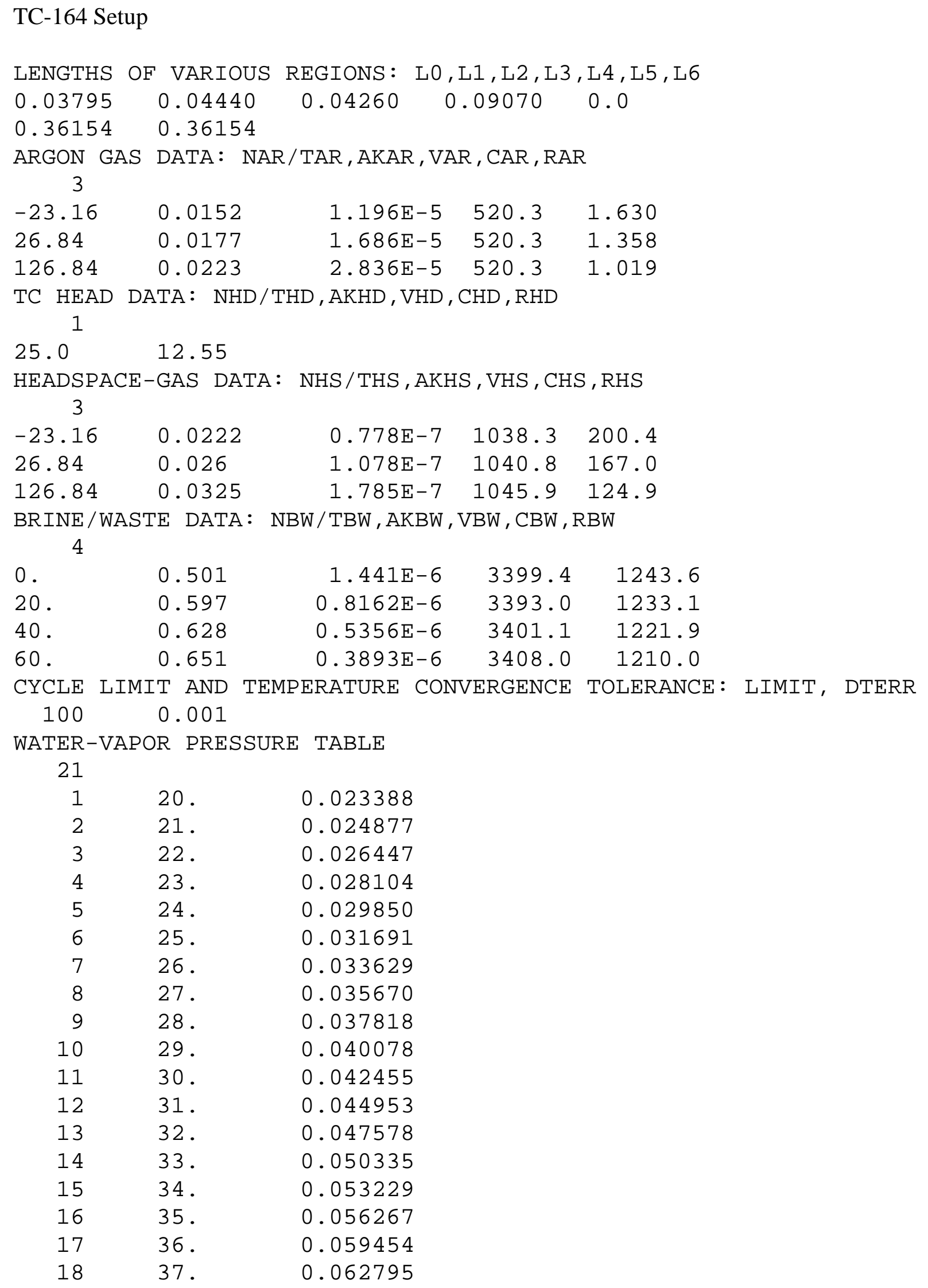




$\begin{array}{lll}19 & 38 . & 0.066298 \\ 20 & 39 . & 0.069969 \\ 21 & 40 . & 0.073814\end{array}$

TEST-CONTAINER VOLUME \& LINEAR THERMAL-EXPANSION COEFFICIIENT 7272. $11.3 E-6$

GLOBAL EXCESS TEMP OF GB INTERIOR VS. THE GB RTD MEASUREMENT $\odot$.

NUMBER OF RTD \& PXD CALIBRATIONS: NCALT, NCALP

85

RTD CALIBRATION INFORMATION: [DATE:HR],PT NO , COMP, RESERR

\begin{tabular}{|c|c|c|c|}
\hline $11-03-95: ? ?$ & $\odot$ & $\odot$. & $\odot$. \\
\hline $07-15-96: ? ?$ & 1962 & $\odot$. & $\odot$. \\
\hline 01-13-97:?? & 6762 & $\odot$. & ○. \\
\hline ०7-09-97:?? & 10817 & $\odot$. & 0.1 \\
\hline 11-21-97:?? & 14037 & $\odot$. & $\odot$. \\
\hline 05-21-98:?? & 18232 & $\odot$. & $\odot$ \\
\hline $11-23-98: ? ?$ & 22596 & $\odot$. & ○. \\
\hline $05-24-99$ :?? & 26743 & $\odot$. & $\odot$. \\
\hline
\end{tabular}

PRESSURE XDUCR CALIBRATIONS: [DATE:HR],PT NO, COMP, RESIDP

12-05-96:?? $5875 \quad 0$.

06-05-97:?? $10007 \quad 0 . \quad 0$.

12-02-97:?? $14290 \quad 0 . \quad 0$.

०6-01-98:?? $18494 \quad 0 . \quad 0$.

12-01-98:?? $22781 \quad 0$.

OVERALL REFERENCE PT DATA: NPTP, PP, TRTDP, TGBP, TGBEXP

$\begin{array}{lllll}10001 & 2167.1 & 30.7 & 28.3 & 2.00\end{array}$

HS-GAS COMPRESSIBILITY TABLE DATA: NUM, VLO, VHI

$50 \quad 0.17 \quad 0.19$

BRINE MASS AND MOLECULAR WT: AMB [G], WTMOLB [G/GMOLE] 7708. 18.5

UNIVERSAL GAS CONSTANT: RU [PSI*ML/GMOLE/K], ATM PRESS[PSIA]

$1205.95 \quad 14.696$

SOL GAS INFO: VOL FRACT[VFRG], MOL WT[WTMOLG], CONSTANTS A, B, C $\odot . \quad-67.3877 \quad 86.3213 \quad 24.7981$

SAMPLE VOLUME: VSAMPL

3.05

NUMBER OF GAS-SAMPLE DATA POINTS: NSAMPL

5

GAS-SAMPLE DATA: [ID], NPTS( I ), PS1, PS2, TRTD, TGB, TGBEX

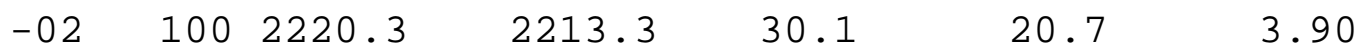

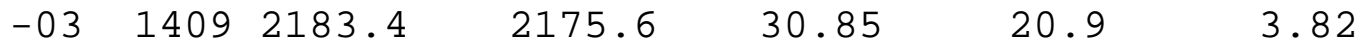

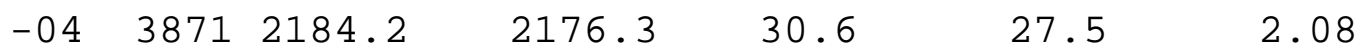

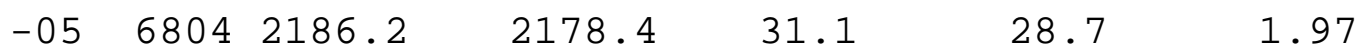

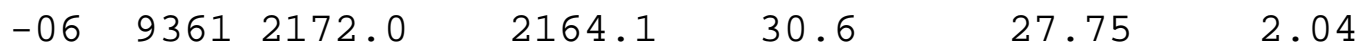

PXD BACKFILL INF0: [N0,PT N0], PC1, PC2, TC1, TC2, TGBC, TGBEXC

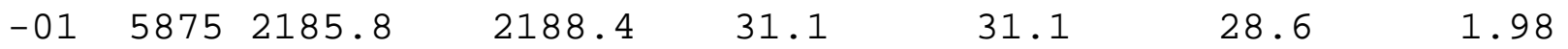




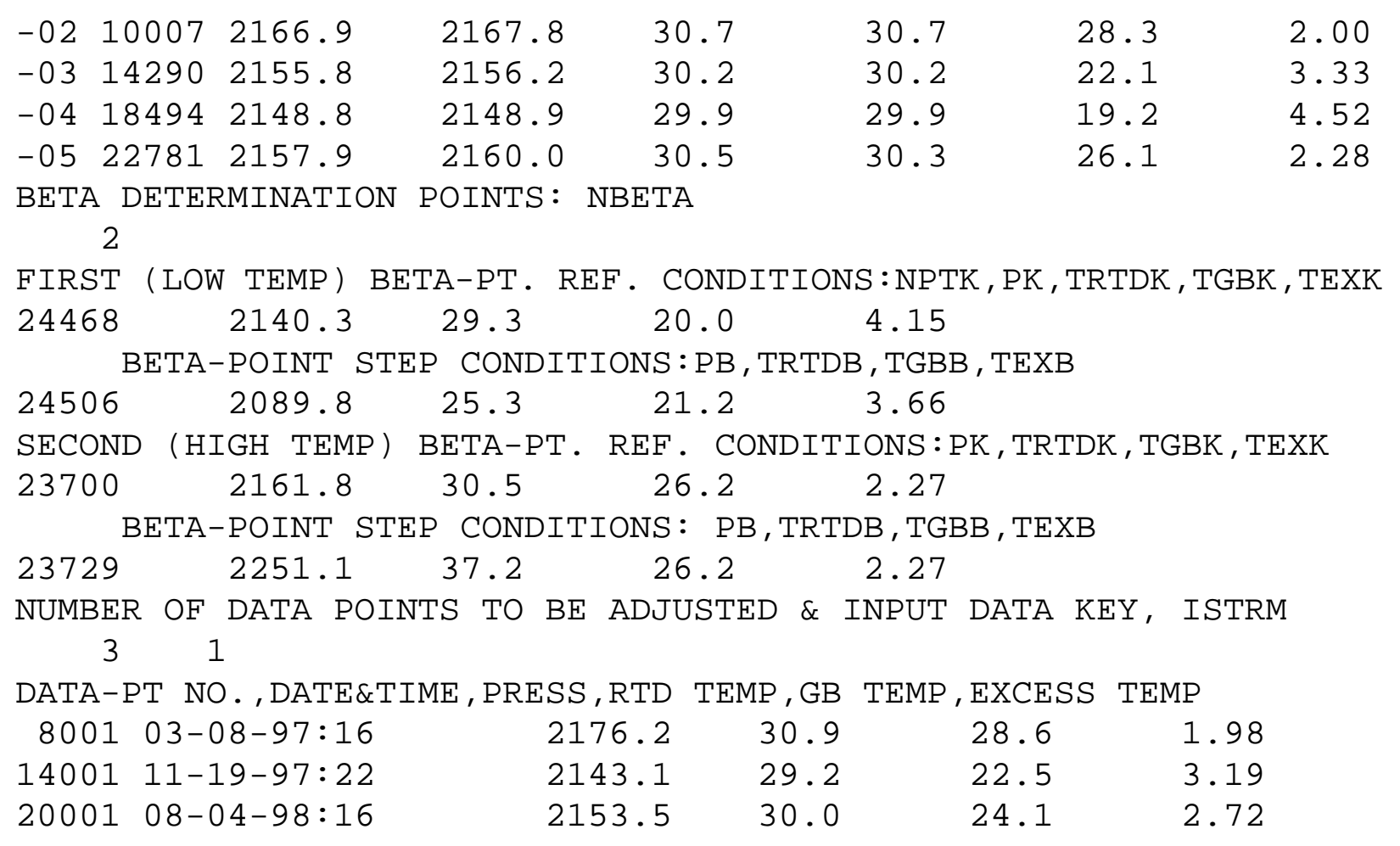




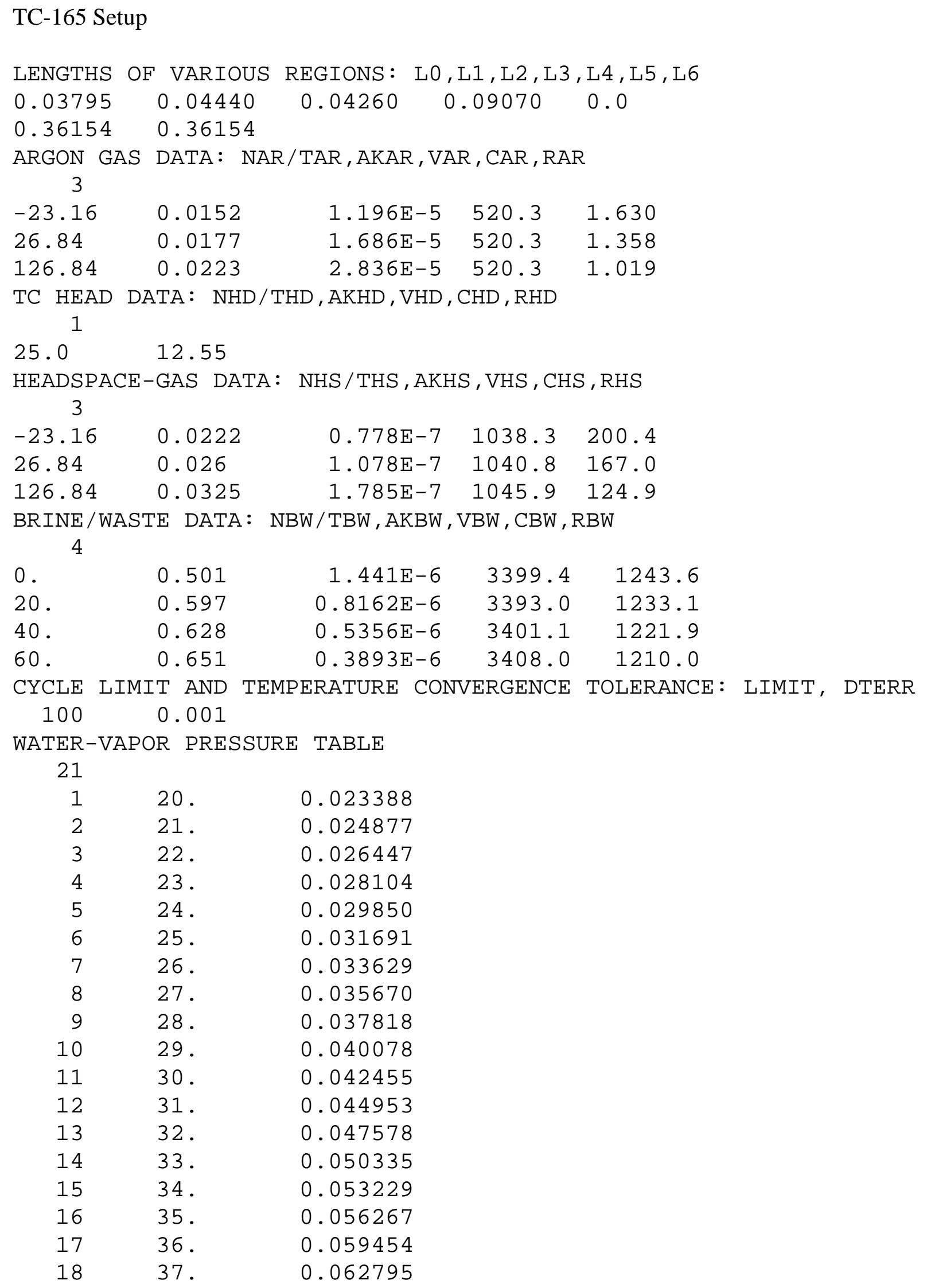




$\begin{array}{lll}19 & 38 . & 0.066298 \\ 20 & 39 . & 0.069969 \\ 21 & 40 . & 0.073814\end{array}$

TEST-CONTAINER VOLUME \& LINEAR THERMAL-EXPANSION COEFFICIIENT 7272. $11.3 E-6$

GLOBAL EXCESS TEMP OF GB INTERIOR VS. THE GB RTD MEASUREMENT $\odot$.

NUMBER OF RTD \& PRESS-XDUCER CALIBRATIONS: NCALT, NCALP 86

RTD CALIBRATION INFORMATION: [DATE:HR],PT NO , COMP, RESERR

$\begin{array}{rrll}11-03-95: ? ? & 0 & 0 . & 0 . \\ 07-15-96: ? ? & 2537 & 0 . & 0 . \\ 01-13-97: ? ? & 6776 & 0 . & 0 . \\ 07-09-97: ? ? & 10836 & 0 . & 0.1 \\ 11-21-97: ? ? & 14045 & 0 . & 0 . \\ 05-21-98: ? ? & 18323 & 0 . & -0.1 \\ 11-23-98: ? ? & 22682 & 0 . & 0 . \\ 05-24-99: ? ? & 26842 & 0 . & 0.1\end{array}$

PRESSURE-XDUCER CALIBRATION INFO: [DATE:HR], PT NO., COMP., RESIDP

$\begin{array}{rrll}\odot 9-19-96: \odot 9 & 4066 & \odot . & \odot . \\ \odot 3-19-97: ? ? & 8264 & \odot . & \odot . \\ \odot 9-19-97: ? ? & 12546 & \odot . & \odot . \\ \odot 2-20-98: ? ? & 16195 & \odot . & \odot . \\ \odot 8-19-98: ? ? & 20439 & \odot . & \odot . \\ \odot 2-17-99: ? ? & 24582 & \odot . & \odot .\end{array}$

OVERALL REFERENCE PT DATA: NPTP, PP, TRTDP, TGBP, TGBEXP

$\begin{array}{lllll}10001 & 2142.0 & 30.6 & 28.4 & 2.00\end{array}$

HS-GAS COMPRESSIBILITY TABLE DATA: NUM, VLO, VHI

$50 \quad 0.17 \quad 0.19$

BRINE MASS AND MOLECULAR WEIGHT: AMB [G],WTMOLB [G/GMOLE]

7708. $\quad 18.5$

UNIVERSAL GAS CONSTANT: RU [PSI*ML/GMOLE/K], ATM PRESS [PSIA] $1205.95 \quad 14.696$

SOL GAS INFO: VOL FRACT[VFRG], MOL WT[WTMOLG], CONSTANTS A, B, C

$\odot . \quad-67.3877 \quad 86.3213 \quad 24.7981$

SAMPLE VOLUME: VSAMPL

3.05

NUMBER OF GAS-SAMPLE DATA POINTS: NSAMPL

5

GAS-SAMPLE DATA: [ ID ], NPTS( I ), PS1, PS2, TRTD, TGB, TGBEX

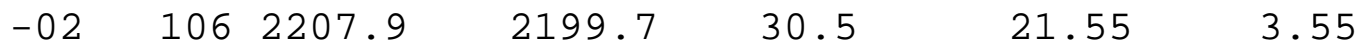

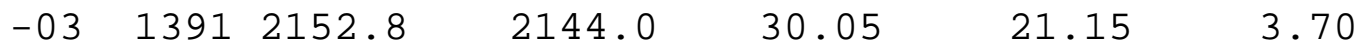

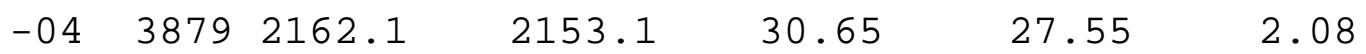

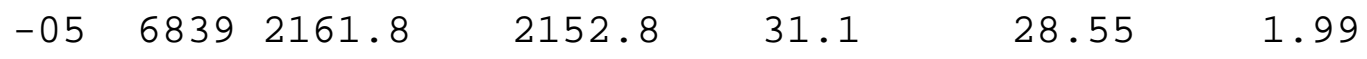

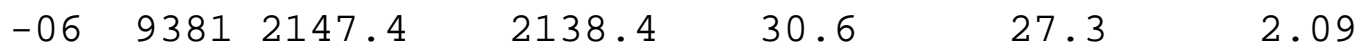

PXD BACKFILL INF0: [NO.,PT.NO.],PC1,PC2, TC1, TC2, TGBC, TGBEXC 


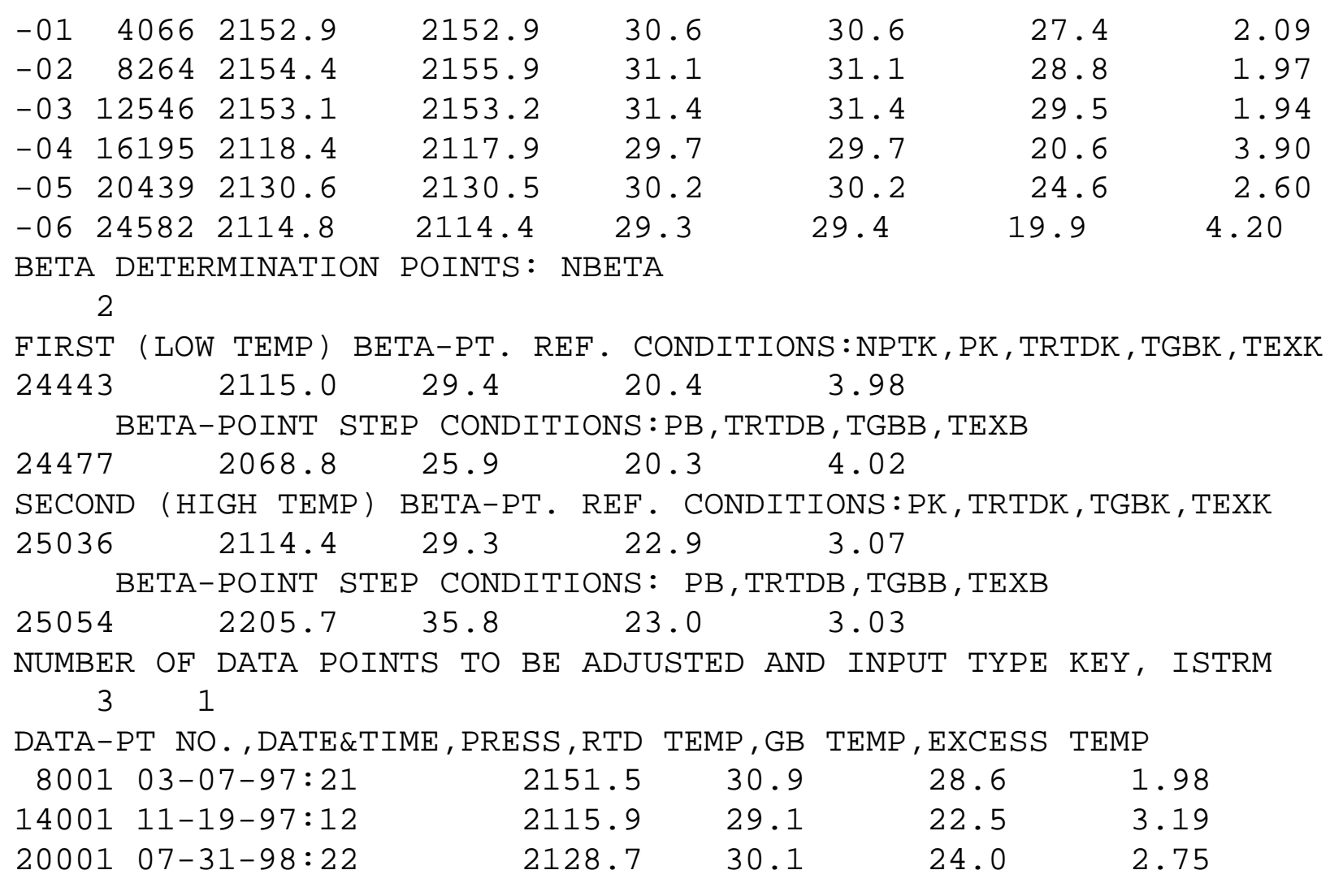




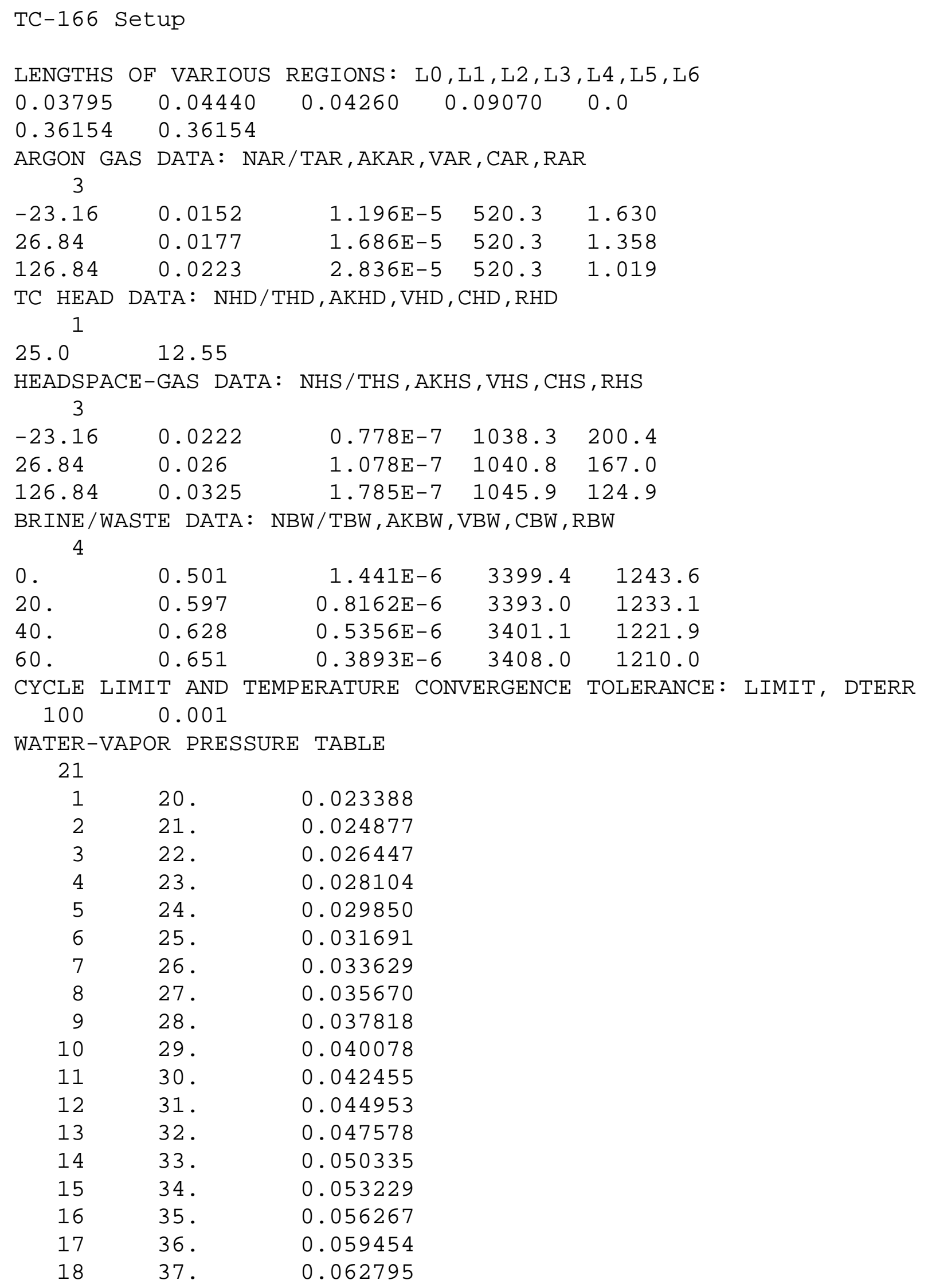




$\begin{array}{lll}19 & 38 . & 0.066298 \\ 20 & 39 . & 0.069969 \\ 21 & 40 . & 0.073814\end{array}$

TEST-CONTAINER VOLUME \& LINEAR THERMAL-EXPANSION COEFFICIIENT 7272. $11.3 E-6$

GLOBAL EXCESS TEMP OF GB INTERIOR VS. THE GB RTD MEASUREMENT $\odot$.

NUMBER OF RTD \& PXD CALIBRATIONS: NCALT, NCALP

$8 \quad 7$

RTD CALIBRATION INFORMATION: [DATE:HR],PT NO , COMP, RESERR

$\begin{array}{rrll}11-03-95: ? ? & 1 & \odot . & -0.1 \\ 07-15-96: ? ? & 2280 & \odot . & -0.1 \\ 01-13-97: ? ? & 6520 & \odot . & \odot .1 \\ 07-09-97: ? ? & 10580 & \odot . & 0.1 \\ 11-21-97: ? ? & 13799 & \odot . & \odot . \\ 05-21-98: ? ? & 18070 & \odot . & \odot . \\ 11-23-98: ? ? & 22431 & \odot . & \odot .1 \\ 05-24-99: ? ? & 2640 \odot & \odot . & 0.1\end{array}$

PRESSURE-XDUCER CALIBRATION INFO: [DATE:HR],PT NO, COMP, RESIDP

\begin{tabular}{|c|c|c|}
\hline ๑4- -1-96:?? & 1 & $\odot$. \\
\hline $06-13-96: ? ?$ & 1664 & $\odot$. \\
\hline $12-05-96: ? ?$ & 5628 & $\odot$. \\
\hline ๑6-๑5-97:?? & 9773 & $\odot$. \\
\hline $12-02-97: ? ?$ & 14052 & 0 . \\
\hline 06-01-98:?? & 18329 & $\odot$. \\
\hline $12-01-98: ? ?$ & 22434 & $\odot$. \\
\hline
\end{tabular}

OVERALL REFERENCE PT DATA: NPTP, PP, TRTDP, TGBP, TGBEXP

$\begin{array}{lllll}10001 & 2152.7 & 31.0 & 28.6 & 1.98\end{array}$

HS-GAS COMPRESSIBILITY TABLE DATA: NUM, VLO, VHI

$50 \quad 0.17 \quad 0.19$

BRINE MASS AND MOLECULAR WEIGHT: AMB[G], WTMOLB[G/GMOLE]

7548. 18.5

UNIVERSAL GAS CONSTANT: RU[PSI*ML/GMOLE/K], STD ATM PRESS[PSIA] $1205.95 \quad 14.696$

SOLUBLE-GAS INFO:VFRG(VOL FRACT), WTMOLG(MOL WT), CONSTANTS A, B, C $\odot . \quad-67.3877 \quad 86.3213 \quad 24.7981$

SAMPLE VOLUME: VSAMPL

3.05

NUMBER OF GAS-SAMPLE DATA POINTS: NSAMPL

5

GAS-SAMPLE DATA: [ ID ], NPTS( I ) , PS1, PS2, TRTD, TGB, TGBEX

$\begin{array}{lrlllll}-02 & 26 & 2197.4 & 2188.1 & 30.9 & 21.4 & 3.58 \\ -03 & 149 \odot & 2168.3 & 2158.9 & 30.6 & 22.2 & 3.29 \\ -04 & 3948 & 2161.4 & 2152.2 & 30.3 & 27.6 & 2.07 \\ -05 & 6675 & 2168.8 & 2159.3 & 31.2 & 28.5 & 1.99 \\ -06 & 9241 & 2151.6 & 2142.5 & 30.5 & 28.3 & 2.00\end{array}$


PXD BACKFILL INF0: [NO, PT] , PC1, PC2, TC1, TC2, TGBC, TGBEXC

\begin{tabular}{|c|c|c|c|c|c|c|c|}
\hline-01 & 1 & 2130.2 & 2130.2 & 25.5 & 25.5 & 20.5 & 3.94 \\
\hline$-\odot 2$ & 1664 & 2156.9 & 2156.9 & 30.5 & 30.5 & 21.6 & 3.51 \\
\hline$-\odot 3$ & 5628 & 2165.4 & 2166.9 & 31.0 & 31.0 & 28.6 & 1.98 \\
\hline$-\odot 4$ & 9773 & 2145.8 & 2146.3 & 30.6 & 30.6 & 27.7 & 2.06 \\
\hline-05 & 14052 & 2145.5 & 2145.0 & 30.7 & 30.7 & 22.1 & 3.33 \\
\hline-06 & 18329 & 2145.0 & 2145.0 & 30.7 & 30.7 & 19.5 & 4.38 \\
\hline ) & 22434 & 2164.4 & 2166.5 & 30.5 & 30.6 & 26.0 & 2.30 \\
\hline
\end{tabular}

BETA DETERMINATION POINTS: NBETA

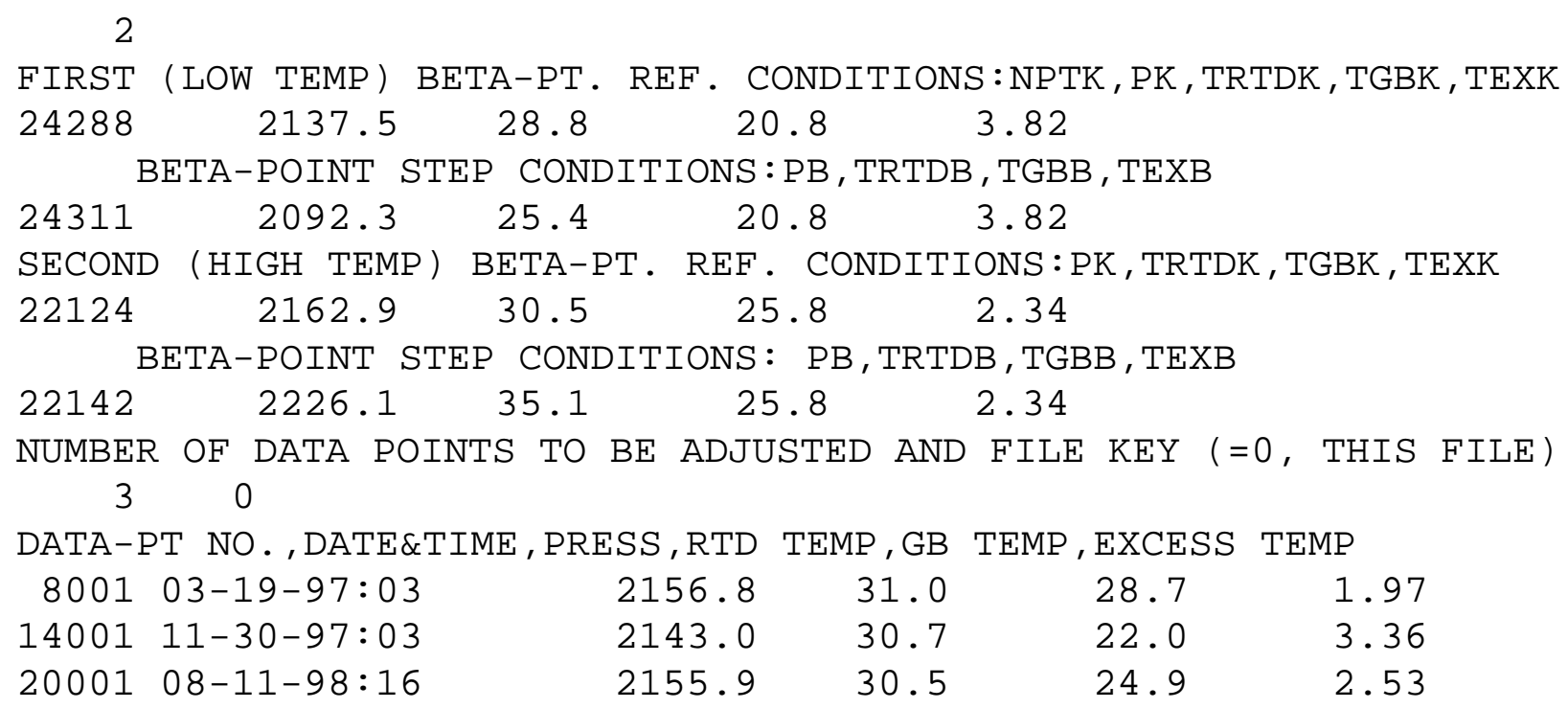




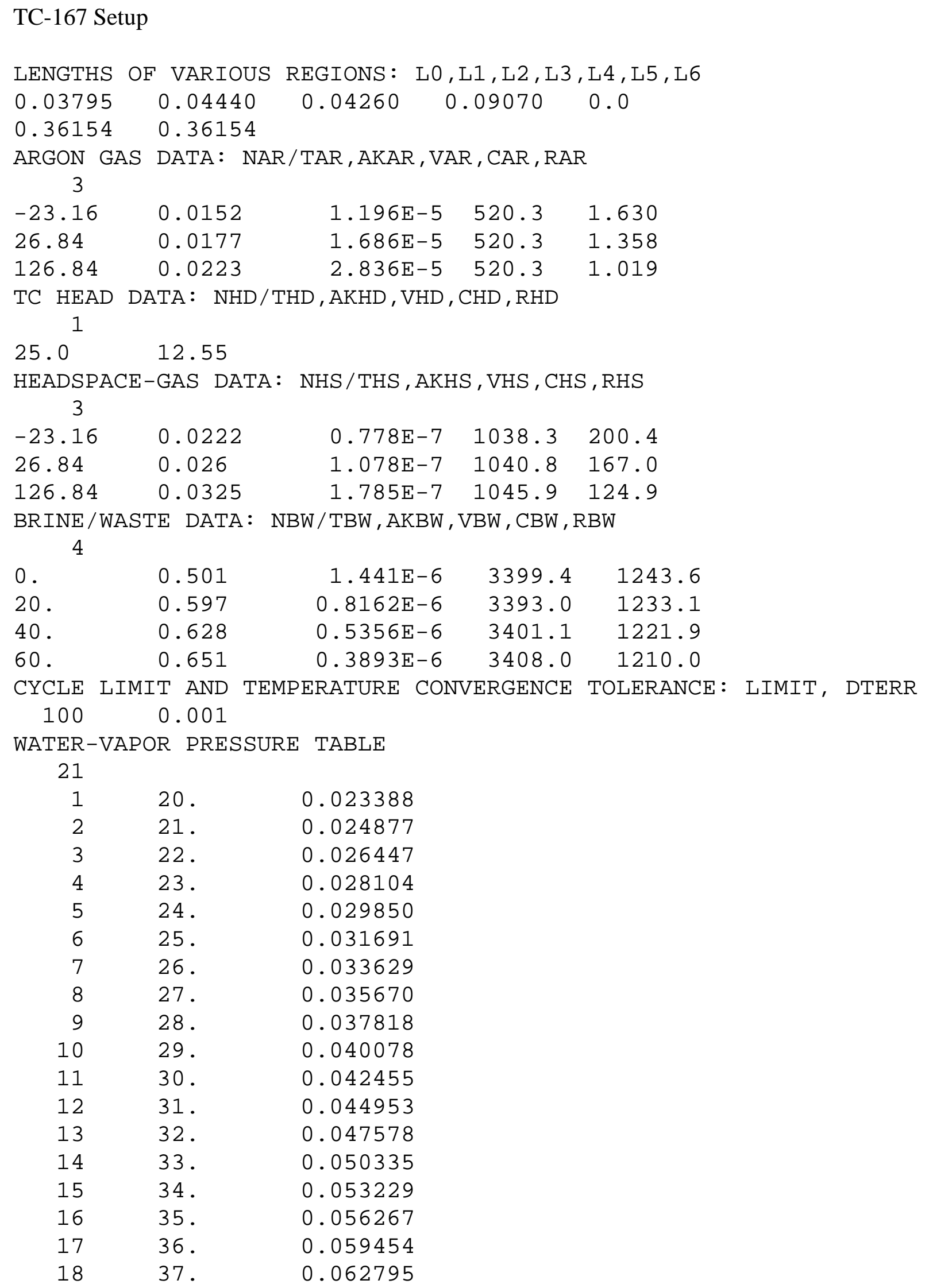




$\begin{array}{lll}19 & 38 . & 0.066298 \\ 20 & 39 . & 0.069969 \\ 21 & 40 . & 0.073814\end{array}$

TEST-CONTAINER VOLUME \& LINEAR THERMAL-EXPANSION COEFFICIIENT 7272. $11.3 E-6$

GLOBAL EXCESS TEMP OF GB INTERIOR VS. THE GB RTD MEASUREMENT $\odot$.

NUMBER OF RTD \& PXD CALIBRATIONS: NCALT, NCALP

86

RTD CALIBRATION INFORMATION: [DATE:HR],PT NO , COMP, RESERR

\begin{tabular}{|c|c|c|c|}
\hline $11-03-95: ? ?$ & $\odot$ & $\odot$. & $-\odot$ \\
\hline $07-15-96: ? ?$ & 2148 & 0. & $-\odot$ \\
\hline 01-13-97:?? & 6399 & O. & ○. \\
\hline ०7- -9 - 97:?? & 10450 & $\odot$. & $\odot$. \\
\hline 11-21-97:?? & 13666 & $\odot$. & $\odot$ \\
\hline $05-21-98: ? ?$ & 17946 & 0. & $\odot$ \\
\hline $11-23-98: ? ?$ & 22312 & ○. & $\odot$ \\
\hline 05-24-99:?? & 26488 & $\odot$. & $\odot$ \\
\hline PRESSURE XDUCR & CALIB & $\mathrm{DN} I$ & DAT \\
\hline $04-08-96: 14$ & 1 & 0. & $\odot$ \\
\hline $10-03-96: ? ?$ & 4006 & 0. & $\odot$ \\
\hline ०4- -3-97: ?? & 8230 & $\odot$. & $\odot$ \\
\hline $12-01-97: ? ?$ & 13899 & $\odot$. & $\odot$ \\
\hline 06-01-98:?? & 18207 & $\odot$. & $\odot$ \\
\hline $12-01-98: ? ?$ & 22499 & $\odot$. & $\odot$ \\
\hline
\end{tabular}

OVERALL REFERENCE PT DATA: NPTP, PP, TRTDP, TGBP, TGBEXP

$\begin{array}{lllll}10001 & 2169.6 & 31.2 & 28.8 & 1.97\end{array}$

HS-GAS COMPRESSIBILITY TABLE DATA: NUM, VLO, VHI

$50 \quad 0.17 \quad 0.19$

BRINE MASS AND MOLECULAR WEIGHT: AMB[G], WTMOLB[G/GMOLE]

7161. $\quad 18.5$

UNIVERSAL GAS CONSTANT: RU[PSI*ML/GMOLE/K], STD ATM PRESS[PSIA] $1205.95 \quad 14.696$

SOL-GAS INFO: VFRG(VOL FRACT), WTMOLG(MOL WT), CONSTANTS A, B, C

$\odot . \quad-67.3877 \quad 86.3213 \quad 24.7981$

SAMPLE VOLUME: VSAMPL

3.05

NUMBER OF GAS-SAMPLE DATA POINTS: NSAMPL

5

GAS-SAMPLE DATA: [ID] , NPTS( I ), PS1, PS2, TRTD, TGB, TGBEX

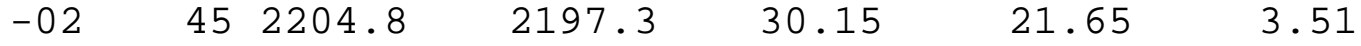

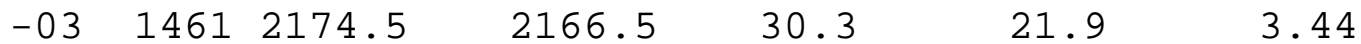

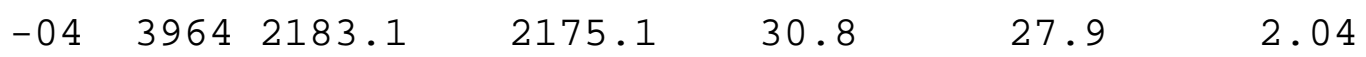

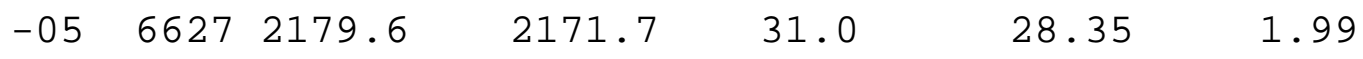

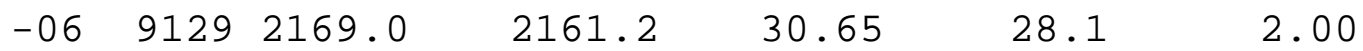

PXD BACKFILL INF0: [N0,PT NO], PC1, PC2, TC1, TC2, TGBC, TGBEXC 


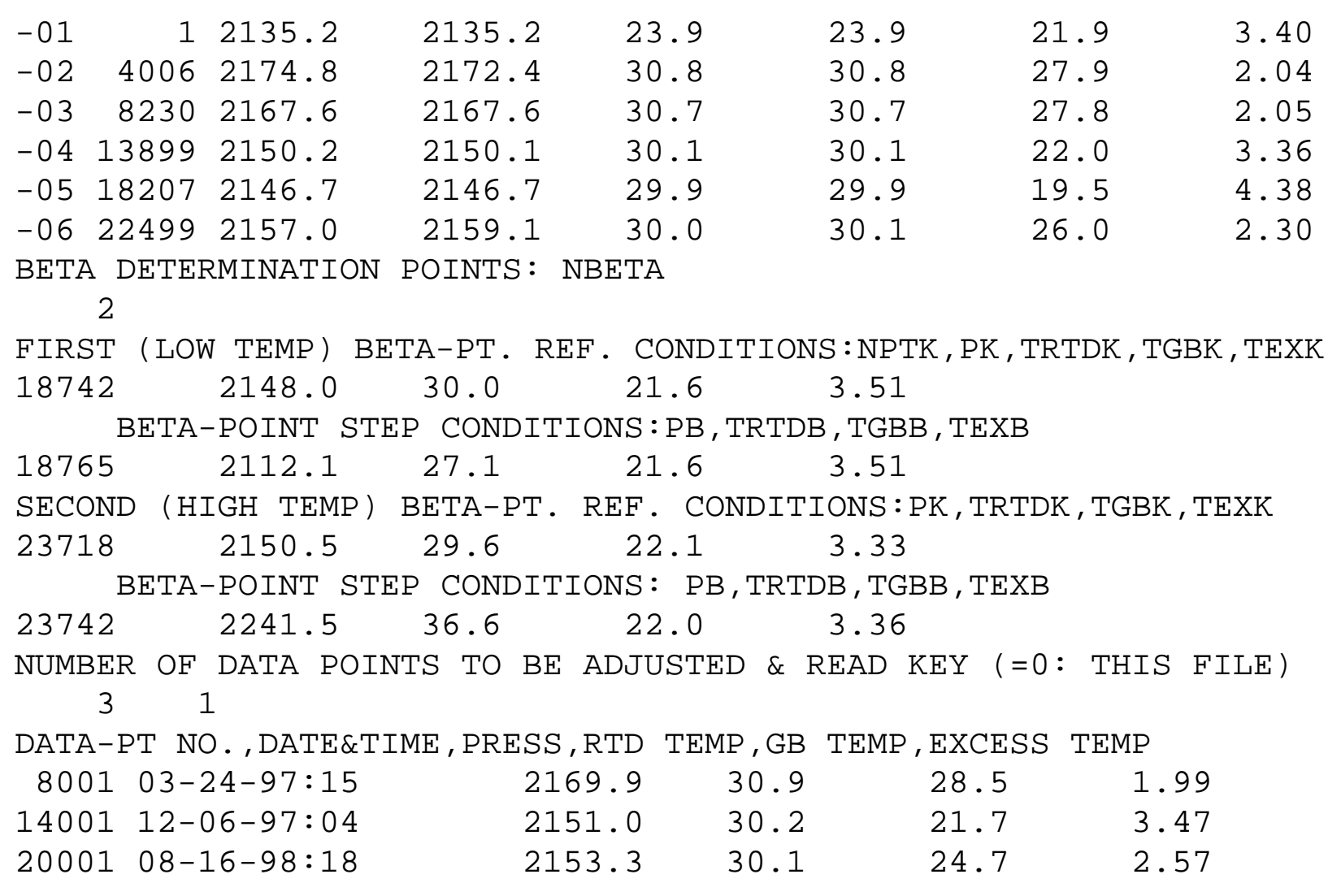




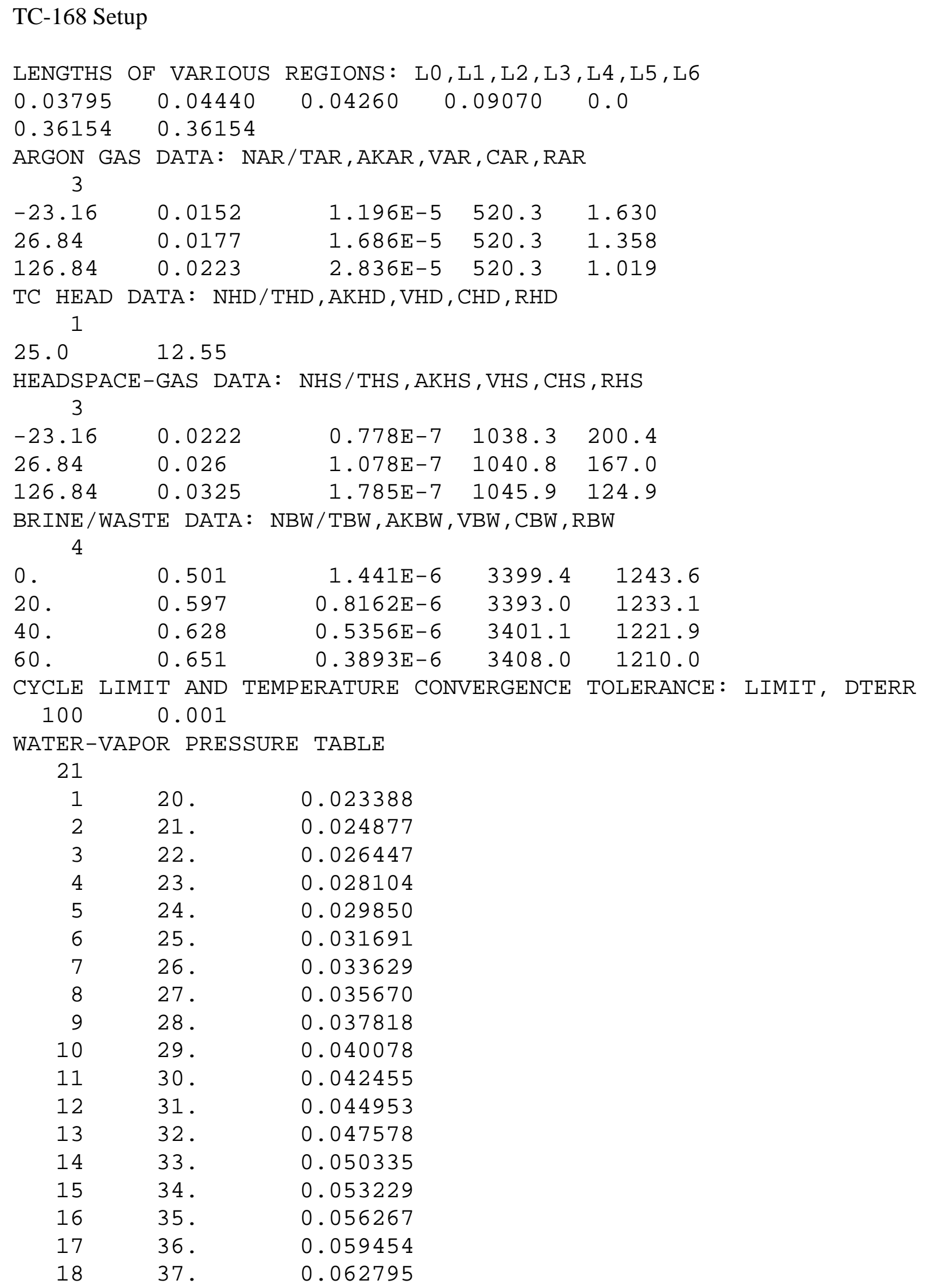




$\begin{array}{lll}19 & 38 . & 0.066298 \\ 20 & 39 . & 0.069969 \\ 21 & 40 . & 0.073814\end{array}$

TEST-CONTAINER VOLUME \& LINEAR THERMAL-EXPANSION COEFFICIIENT 7272. $11.3 E-6$

GLOBAL EXCESS TEMP OF GB INTERIOR VS. THE GB RTD MEASUREMENT $\odot$.

NUMBER OF RTD \& PXD CALIBRATIONS: NCALT, NCALP

86

RTD CALIBRATION INFORMATION: [DATE:HR],PT NO , COMP, RESERR

$\begin{array}{rrll}\text { 11-03-95:?? } & 0 & 0 . & 0 . \\ 07-15-96: ? ? & 2057 & 0 . & -0.1 \\ 01-13-97: ? ? & 6305 & 0 . & \odot . \\ 07-09-97: ? ? & 10368 & 0 . & 0 . \\ 11-21-97: ? ? & 13576 & -0.1 & 0 . \\ 05-21-98: ? ? & 17830 & 0 . & 0.1 \\ 11-23-98: ? ? & 22187 & 0 . & 0 . \\ 05-24-99: ? ? & 26376 & 0 . & 0.1\end{array}$

PRESSURE-XDUCR CALIBRATION INFO: [DATE:HR],PT NO, COMP, RESIDP

$\begin{array}{rrrl}04-15-96: ? ? & 69 & 0 . & 0 . \\ 10-03-96: ? ? & 3919 & \odot . & 0 . \\ 04-03-96: ? ? & 8148 & \odot . & \odot . \\ 12-01-97: ? ? & 13808 & \odot . & 0 . \\ 06-01-98: ? ? & 18093 & \odot . & \odot . \\ 12-01-98: ? ? & 22373 & \odot . & 0 .\end{array}$

OVERALL REFERENCE PT DATA: NPTP, PP, TRTDP, TGBP, TGBEXP

$\begin{array}{lllll}10001 & 2146.5 & 31.3 & 28.9 & 1.96\end{array}$

HS-GAS COMPRESSIBILITY TABLE DATA: NUM, VLO, VHI

$50 \quad 0.17 \quad 0.19$

BRINE MASS AND MOLECULAR WEIGHT: AMB[G], WTMOLB[G/GMOLE]

7416. $\quad 18.5$

UNIVERSAL GAS CONSTANT: RU[PSI*ML/GMOLE/K], STD ATM PRESS[PSIA] $1205.95 \quad 14.696$

SOL-GAS INFO:VFRG(VOL FRACT), WTMOLG(MOL WT), CONSTANTS A, B, C

$\odot . \quad-67.3877 \quad 86.3213 \quad 24.7981$

SAMPLE VOLUME: VSAMPL

3.05

NUMBER OF GAS-SAMPLE DATA POINTS: NSAMPL

5

GAS-SAMPLE DATA: [ID], NPTS( I ), PS1, PS2, TRTD, TGB, TGBEX

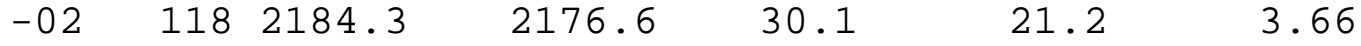

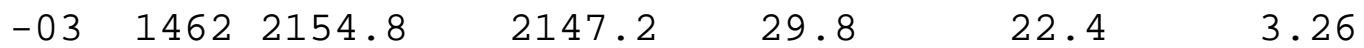

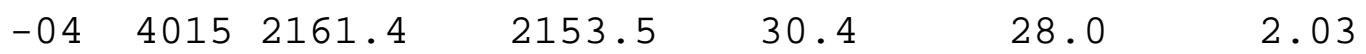

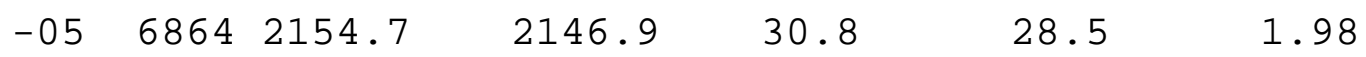

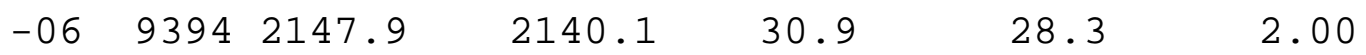

PXD BACKFILL INF0: [N0.,PT N0], PC1, PC2, TC1, TC2, TGBC, TGBEXC 


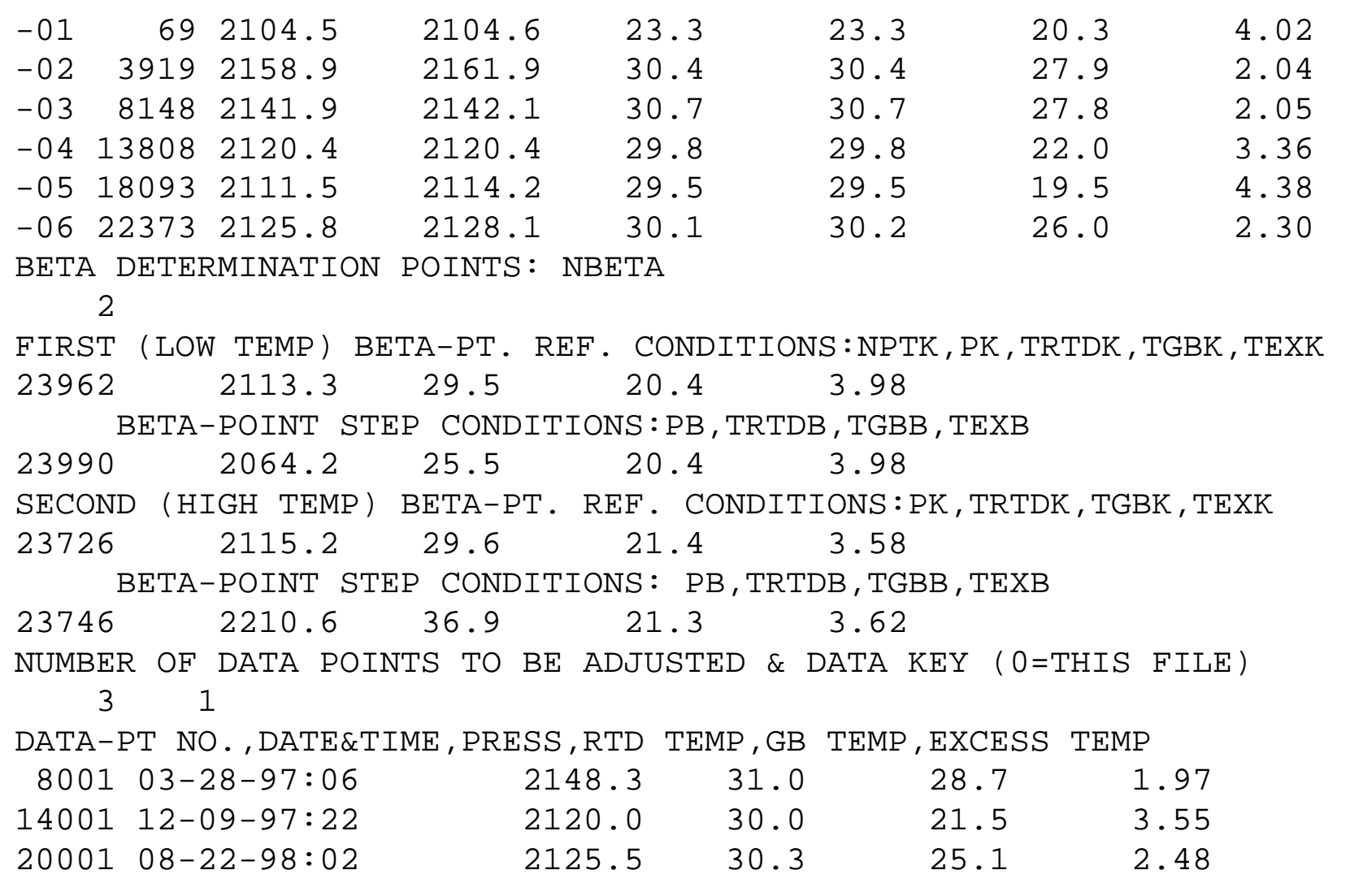




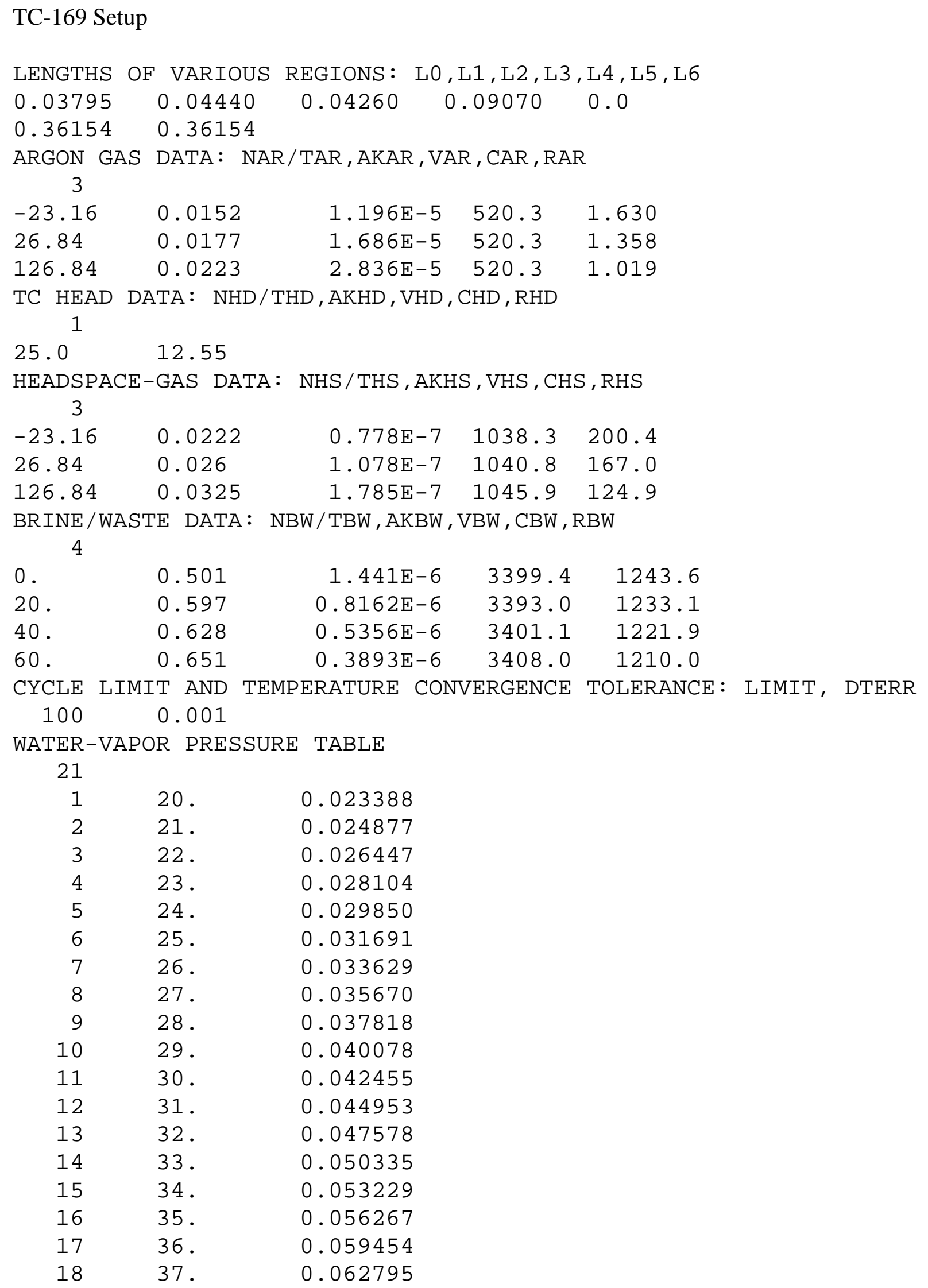




$\begin{array}{lll}19 & 38 . & 0.066298 \\ 20 & 39 . & 0.069969 \\ 21 & 40 . & 0.073814\end{array}$

TEST-CONTAINER VOLUME \& LINEAR THERMAL-EXPANSION COEFFICIIENT 7272. $11.3 E-6$

GLOBAL EXCESS TEMP OF GB INTERIOR VS. THE GB RTD MEASUREMENT $\odot$.

NUMBER OF RTD AND PRESSURE XDUCER CALIBRATIONS: NCALT, NCALP 85

RTD CALIBRATION INFORMATION: [DATE:HR],PT NO , COMP, RESERR

\begin{tabular}{|c|c|c|c|}
\hline $11-03-95: ? ?$ & $\odot$ & $\odot$. & 0.1 \\
\hline $07-15-96: ? ?$ & 1749 & 0. & 0.1 \\
\hline $01-13-97: ? ?$ & 5997 & $\Theta$. & $\odot$. \\
\hline ०7- -9-97:?? & 10053 & $\odot$. & 0.1 \\
\hline $11-21-97: ? ?$ & 13266 & 0.1 & $\odot$. \\
\hline 05-21-98:?? & 17550 & $\odot$. & 0.1 \\
\hline $11-23-98: ? ?$ & 21920 & ○. & 0.1 \\
\hline 05-24-98:?? & 26101 & ○. & 0.1 \\
\hline
\end{tabular}

PRESSURE-XDUCER CALIBRATION INFO: [DATE:HR], PT NO, COMP, RESIDP

$\begin{array}{rrrr}10-03-96: ? ? & 3608 & 0 . & \odot . \\ \odot 4-03-97: ? ? & 7841 & \odot . & \odot . \\ 12-02-97: ? ? & 13521 & \odot . & \odot . \\ \odot 6-\odot 1-98: ? ? & 17809 & \odot . & \odot . \\ 12-01-98: ? ? & 22108 & \odot . & \odot .\end{array}$

OVERALL REFERENCE PT DATA: NPTP, PP, TRTDP, TGBP, TGBEXP

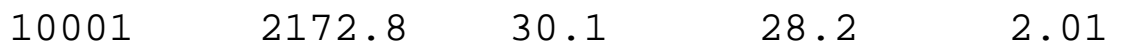

HS-GAS COMPRESSIBILITY TABLE DATA: NUM, VLO, VHI

$$
50 \quad 0.17 \quad 0.19
$$

BRINE MASS AND MOLECULAR WEIGHT: AMB[G],WTMOLB[G/GMOLE]

7472. 18.5

UNIVERSAL GAS CONSTANT: RU[PSI(ML/GMOLE/K], STD ATM PRESS[PSIA]

1205.9514 .696

SOL-GAS INFO: VFRG(VOL FRACT), WTMOLG(MOL WT), CONSTANTS A, B, C $\odot . \quad-67.3877 \quad 86.3213 \quad 24.7981$

SAMPLE VOLUME: VSAMPL

3.05

NUMBER OF GAS-SAMPLE DATA POINTS: NSAMPL

4

GAS-SAMPLE DATA: [ID] , NPTS( I ), PS1, PS2, TRTD, TGB, TGBEX

$\begin{array}{lrllllll}-02 & 136 & 2196.1 & 2187.3 & 30.0 & 20.25 & 4.07 \\ -03 & 2706 & 2198.1 & 2189.6 & 30.5 & 27.35 & 2.09 & \\ -04 & 5389 & 2203.4 & 2195.0 & 31.1 & 28.85 & 1.97 \\ -05 & 7901 & 2188.4 & 2180.0 & 30.7 & 27.9 & 2.04 & \\ \text { PXD } & \text { BACKFILL INF0: }[\text { NO, PT NO, ], PC1,PC2, TC1, TC2, TGBC, TGBEXC } & \\ -01 & 3608 & 2192.0 & 2195.8 & 30.6 & 30.6 & 27.7 & 2.06 \\ -02 & 7841 & 2184.9 & 2184.7 & 30.5 & 30.5 & 27.2 & 2.12\end{array}$




$\begin{array}{llllllll}-03 & 13521 & 2167.1 & 2166.9 & 30.1 & 30.1 & 22.1 & 3.33 \\ -04 & 17809 & 2160.1 & 2161.7 & 29.9 & 29.9 & 19.5 & 4.38 \\ -05 & 22108 & 2170.6 & 2172.2 & 30.2 & 30.2 & 26.0 & 2.30\end{array}$

BETA DETERMINATION POINTS: NBETA

2

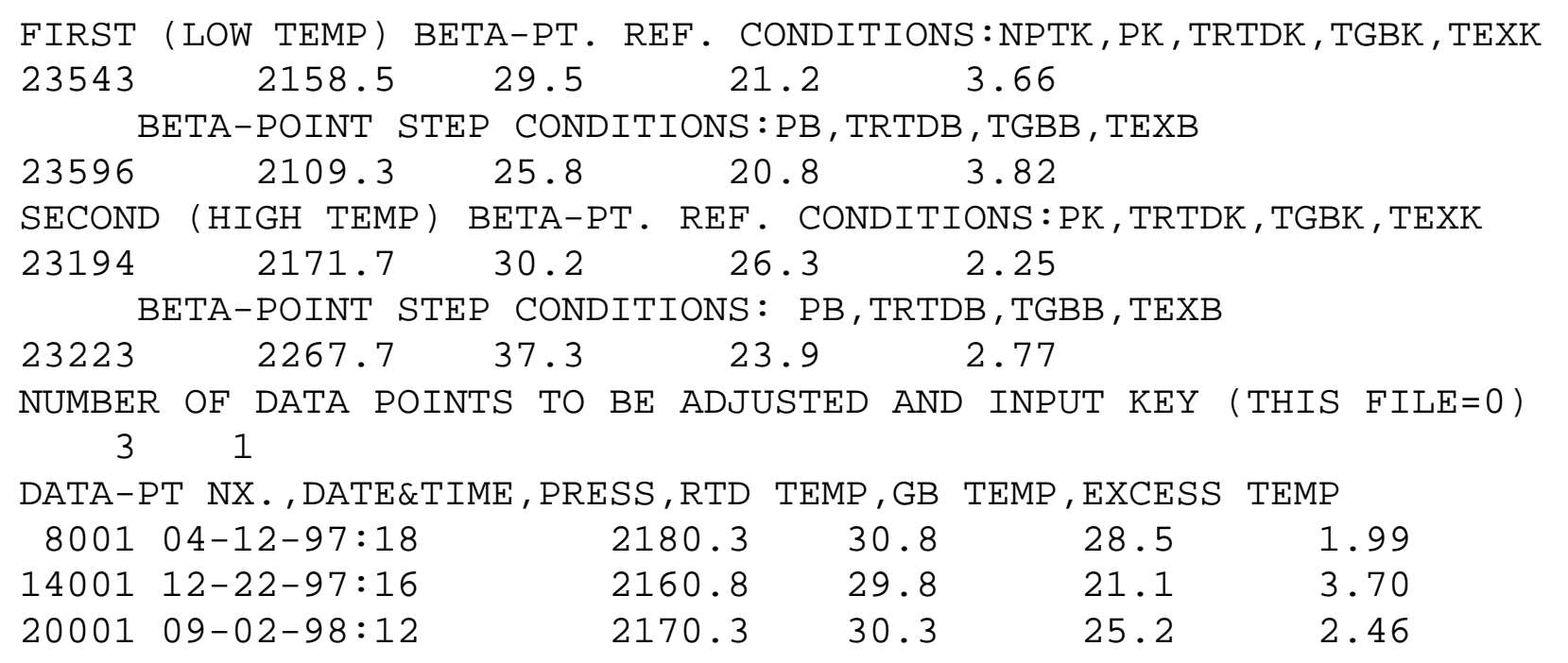




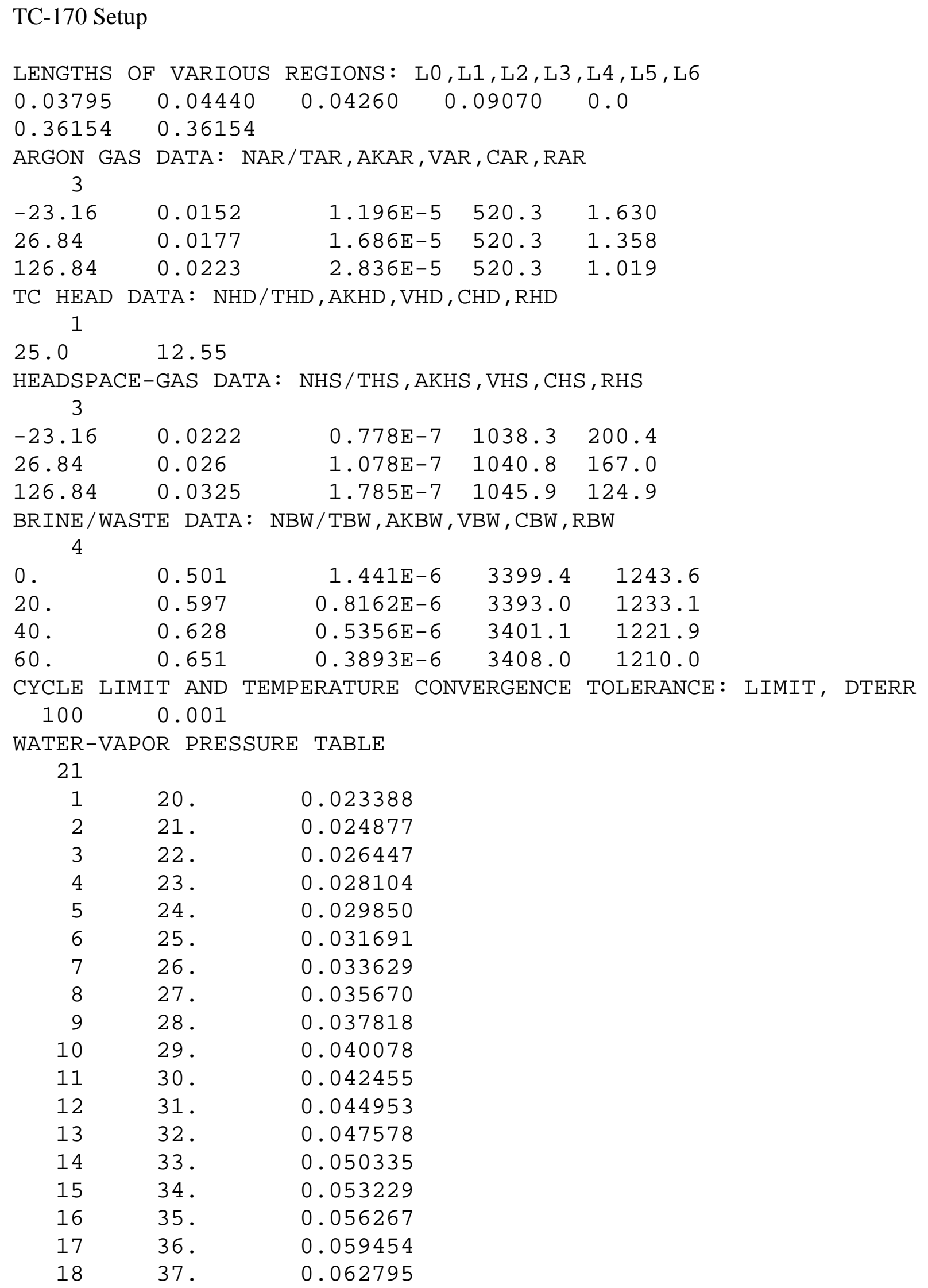




$\begin{array}{lll}19 & 38 . & 0.066298 \\ 20 & 39 . & 0.069969 \\ 21 & 40 . & 0.073814\end{array}$

TEST-CONTAINER VOLUME \& LINEAR THERMAL-EXPANSION COEFFICIIENT 7272. $11.3 E-6$

GLOBAL EXCESS TEMP OF GB INTERIOR VS. THE GB RTD MEASUREMENT $\odot$.

NUMBER OF RTD \& PRESS XDUCER CALIBRATIONS: NCALT, NCALP $8 \quad 7$

RTD CALIBRATION INFORMATION: [DATE:HR],PT NO., COMP, RESERR

\begin{tabular}{|c|c|c|c|}
\hline $11-03-95: ? ?$ & $\odot$ & $\odot$. & 0.1 \\
\hline $07-15-96: ? ?$ & 1233 & ○. & 0.1 \\
\hline 01-13-97:?? & 5481 & $\odot$. & $\odot$. \\
\hline ०7- -9-97:?? & 9544 & $\odot$. & 0.1 \\
\hline $11-21-97: ? ?$ & 12758 & -0.1 & $\odot$. \\
\hline 05-21-98:?? & 17044 & $\odot$. & 0.1 \\
\hline $11-23-98: ? ?$ & 21413 & $\odot$. & 0.1 \\
\hline ०5-24-99:?? & 25578 & $\odot$. & 0.2 \\
\hline
\end{tabular}

PRESSURE XDUCER CALIBRATION INFO:[DATE:HR],PT NO, COMP, RESIDP

\begin{tabular}{|c|c|c|}
\hline $05-15-96: ? ?$ & 21 & $\odot$. \\
\hline $05-31-96: ? ?$ & 324 & $\odot$ \\
\hline $11-14-96: ? ?$ & 4095 & $\odot$. \\
\hline 05-14-97:?? & 8227 & $\odot$. \\
\hline $12-02-97: ? ?$ & 13012 & $\odot$ \\
\hline $06-02-98: ? ?$ & 17320 & $\odot$. \\
\hline $12-07-98: ? ?$ & 21737 & $\odot$. \\
\hline
\end{tabular}

OVERALL REFERENCE PT DATA: NPTP, PP, TRTDP, TGBP, TGBEXP

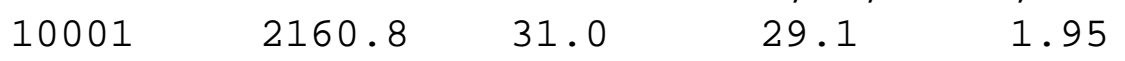

HS-GAS COMPRESSIBILITY TABLE DATA: NUM, VLO, VHI

$50 \quad 0.17 \quad 0.19$

BRINE MASS AND MOLECULAR WEIGHT: AMB[G],WTMOLB[G/GMOLE]

7638. 18.5

UNIVERSAL GAS CONSTANT: RU[PSI*ML/GMOLE/K], STD ATM PRESS[PSIA] $1205.95 \quad 14.696$

SOL-GAS INFO: VFRG (VOL FRACT), WTMOLG (MOL WT), CONSTANTS A, B, C $\odot . \quad-67.3877 \quad 86.3213 \quad 24.7981$

SAMPLE VOLUME: VSAMPL

3.05

NUMBER OF GAS-SAMPLE DATA POINTS: NSAMPL

5

GAS-SAMPLE DATA: [ ID ], NPTS( I ) , PS1, PS2, TRTD, TGB, TGBEX

$\begin{array}{lrlllll}-02 & 45 & 2205.4 & 2195.6 & 30.5 & 21.4 & 3.55 \\ -04 & 419 & 2188.4 & 2178.6 & 30.2 & 22.2 & 3.29 \\ -05 & 2907 & 2178.9 & 2169.3 & 30.5 & 27.6 & 2.07 \\ -06 & 5570 & 2184.1 & 2174.5 & 31.4 & 28.5 & 1.99 \\ -07 & 8184 & 2159.4 & 2150.0 & 30.55 & 27.6 & 2.07\end{array}$


PXD BACKFILL INF0: [NO., PT NO.], PC1, PC2, TC1, TC2, TGBC, TGBEXC

$\begin{array}{rrllllll}-01 & 21 & 2126.7 & 2126.7 & 23.2 & 23.2 & 21.2 & 3.66 \\ -02 & 324 & 2165.0 & 2165.0 & 30.7 & 30.7 & 19.9 & 4.20 \\ -03 & 4095 & 2172.2 & 2174.6 & 30.7 & 30.7 & 28.3 & 2.00 \\ -04 & 8227 & 2152.1 & 2152.8 & 30.5 & 30.5 & 27.8 & 2.05 \\ -05 & 13012 & 2138.6 & 2138.8 & 30.0 & 30.0 & 22.1 & 3.33 \\ -06 & 17320 & 2132.7 & 2133.1 & 29.9 & 29.9 & 19.6 & 4.33 \\ -07 & 21737 & 2147.9 & 2149.1 & 30.5 & 30.5 & 25.9 & 2.32\end{array}$

BETA DETERMINATION POINTS: NBETA

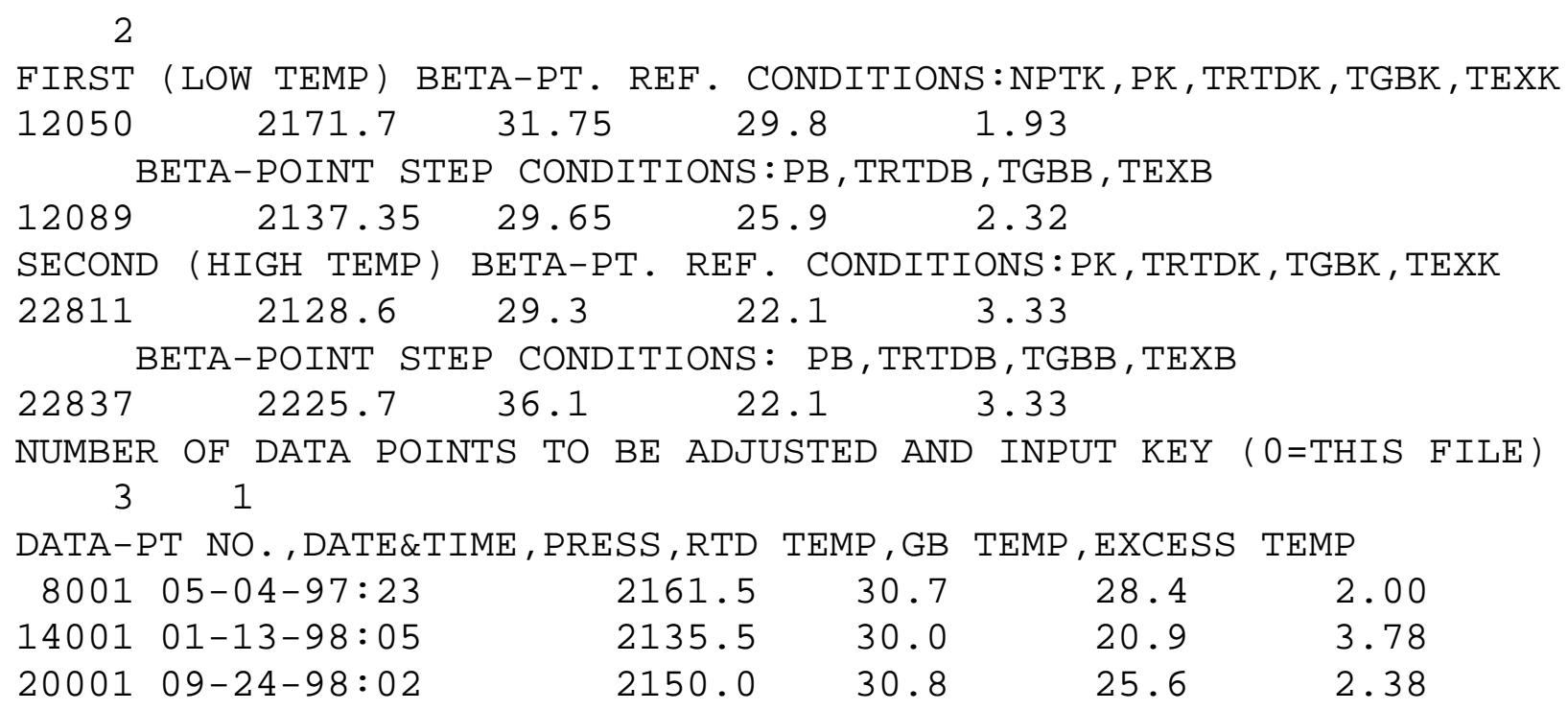




\section{ATTACHMENT 2 TO APPENDIX D}

\section{Streaming-Data-Input Procedure}

(Rev. 03-2002)

1. Add most current data to the EXCEL data file (on the S: drive).

2. Copy GGE Project-generated EXCEL spreadsheet (on the S: drive) to ADJxxx.xls, where xxx denotes the TC number (e.g., 153), for later manipulation.

3. Make the following adjustments to this new EXCEL copy:

a. Make sure that all data are floating points

b. $\quad$ Check that column widths are the standard default of 8.43

c. Delete the "DateValue” column $\left(1^{\text {st }}\right.$ column - A)

d. Format $\mathrm{T}_{\mathrm{RTD}}, \mathrm{T}_{\mathrm{GB}}$, and Pressure column to 1 decimal place

e. Make $T_{\text {excess }}$ calculation, per desired algorithm, and place in $6^{\text {th }}$ column $(F)$, formatted to 2 decimal places

f. $\quad$ Set flags in next 2 columns (G \& H) for

(a) Col. G: duplicate data (time): 1=TRUE; 0=FALSE -- IF(B2=B1,1,0)

(b) Col. H: dropped data: 1=TRUE; 0=FALSE -- IF(OR(C2=0,D2=0),1,0)

Copy these conditional tests to all subsequent rows.

Format these columns to zero decimal places; column width is not important, but if these get too wide the subsequent file has the rows spread out over more than one line

g. In column I set the valid data-point count that omits duplicate and dropped data by placing a " 1 " in cell I2 and in cell I3 test the flags and adjust the count using $\mathrm{IF}(\mathrm{AND}(\mathrm{G} 3=0, \mathrm{H} 3=0), \mathrm{I} 2+1, \mathrm{I} 2)$. Copy this conditional calculation to all data points.

h. Delete any plot sheets, leaving only the data sheet.

After these changes, columns should contain
A date
B time
C B/W RTD Temperature
D GB RTD Temperature
E Measured Pressure 
F Excess temperature calculation per the selected algorithm, e.g., Eqn. 8-5.

G Duplicate data flag, 0 or 1

$\mathrm{H} \quad$ Dropped data flag, 0 or 1

I Valid data count No., i.e., purged of duplicate or dropped data

4. Save the adjusted-count EXCEL file, in case some modification is needed later, e.g., in a CleanDat $\backslash T C-x x x . x l s$ file.

5. Save a copy of the the adjusted, streaming-data input file as a tab-delimited TEXT file as StreamlSTRxxx.TXT for use as input data. When closing, do not save this file as an EXCEL format - i.e., hit the "no" button when asked.

6. Note the number of rows of actual, but raw (not the Valid count) data (i.e., do not include any heading rows).

7. Note the valid count number for all sample events, calibration events, the reference point, the beta points, etc. (The data input to the advanced versions of the GGE program use the valid point numbers.) Note the measured pressure, RTD temp, GB temp, and T-excess values for these points (these will be used in the SetUp data set, SETxxx.txt, originated as a WP file).

8. Open the STRxxx.TXT file in WordPerfect (Courier New, 12 pt font), and make the following changes. Note: the resulting file will be directly input to the FORTRAN application program; the changes must be carefully made to have the correct syntax.

Remove labels \& headings.

The $1^{\text {st }}$ line will be ignored, so this can be used for identification as desired (suggest “TC-153 Streaming Data Input”). The $2^{\text {nd }}$ line must show number of actual (i.e., not including any heading rows) raw, unadjusted data rows, in format $\mathbf{I 5}$. The $3^{\text {rd }}$ row is ignored and can be used as desired (suggest putting a continuous numbering across the page to facilitate confirmation of proper parameter spacing, step 11, below).

Place tabs only at absolute settings of $1,2,3,3-1 / 2,4,5,5-3 / 4,6, \& 6-3 / 8$ in. This should give the following columnization:
a. $\quad$ First 20 characters for date and time
[columns 1-20]
b. $\quad 5$ characters for each of $\mathrm{T}_{\mathrm{RTD}}$ and $\mathrm{T}_{\mathrm{GB}}$
[columns 21-25 \& 26-30]
c. 10 characters for Pressure
[columns 31-40]
d. 7 characters for T-excess
[columns 41-47]
e. 1 character for the duplicate data flag
[column 48]
f. 2 blank spaces
g. 1 character for the dropped data flag
[columns 49-50]
[column 51] 
h. 2 blank spaces

[columns 52-53]

i. 5 characters for the adjusted data count [columns 54-58]

9. Save the file as a DOS unformatted text file, i.e., just hit the "save" icon.

10. Confirm the proper parameter spacing by examining this file in DOS using the TYPE \{filename\}| MORE” command.

Note: further opening of this file in WP will cause it to be in opened in a WPD format - i.e., space-delimited form, where previous tabs are obliterated, so further attempts to move columns by manipulating tabs is useless.

\section{Program Use Procedure}

The GGE7b1 program uses two input files: GGESET.txt and GGESTR.txt, that are copies of the SETxxx.txt and STRxxx.txt files, respectively.

1. Copy the STRxxx.txt file when ready to use to the file, C:|rmfortldataset|GGESTR.txt.

2. Copy the SETxxx.txt file when ready to use to the file, C:Irmfortldataset।GGESET.txt.

3. Execute the desired FORTRAN program version, e.g., GGE7b1.

4. Following the program execution, two output files are created in C:Irmfortloutput:

(1) a summary file of approximately 4 Mbytes for 26,000 data points that gives the results based on the valid data point numbers, C:Irmfortloutput|GGESUM.out (a space-delimited text file); and

(2) a large file of about 48 Mbytes that shows many of the intermediate calculations, primarily for trouble shooting use, C:IrmfortloutputlGGE.out.

5. Open the GGESUM.out file into an EXCEL spreadsheet (“fixed-width” type).

6. Delete or move the first 7 rows, which show

VGP Headspace volume at the Reference Point conditions

TBETA(1) The low temperature for the BETA-Lo parameter

BETA(1) BETA-Lo

TBETA(2) The high temperature for the BETA-Hi parameter

$\operatorname{BETA}(2) \quad$ BETA-Hi

TEXGLB Ignore this parameter

ICYCLE Ignore this parameter

7. Adjust the column widths as desired, and label the columns as follows

$\underline{\text { Column } \quad \underline{\text { Label }}}$ 

A Valid Pt. No.
B Date \& Time
C Measured Pressure
D Adjusted Pressure
E HS Gas Mass
F Vapor Pressure
G Brine/Waste RTD
H Glovebox RTD
I dT-excess
J Est. Local TC Environment
K Mole Fraction of Generated Gas Relative to the Value at Ref. Pt. No. $\{R E F\}$, where $\{R E F\}$ is the number of the point selected as reference and included in the corresponding SETxxx.txt file.

$\mathrm{J}$ is calculated as the sum of the Glovebox RTD plus the dT-excess.

$\mathrm{K}$ is calculated as (Eqn. 32)

$$
\Delta X_{g e n}=\frac{P_{a d j}-P_{0}}{P_{a d j}-P_{v_{\eta}}}
$$

8. Plot the temperatures, pressures, and mole fraction of generated gas columns as line charts.

9. Save this file in Results\TC-Xxx as an EXCEL file for later review, if needed. 


\section{Program Flow Overview \\ Version - GGE7}

MAIN

Set subprogram generalities

Call ONEDIM

Call VAPOR

Call CALIBR

Set Reference Point Conditions T' and P', and set parameters

Call CALIBR ----> corrected T' \& $P$ '

Call ONEDIM----> $\mathrm{T}_{\mathrm{g}}$,

Call VAPOR--.-.-> $\mathrm{P}_{\mathrm{v}}^{\mathrm{g}}$,

Call BB---------> set generalities

Call BB--.----> z'

Call SOLUBL-.--> set generalities, $\mathrm{RN}_{\mathrm{g} \text {-dis }}$,

Call SAMPLE-------> $>\mathrm{R}^{*} \mathrm{dM}_{\mathrm{s}}$

$>$ Sample No. just to left \& right of Reference Pt.

$>\mathrm{V}_{\mathrm{s}}$-term (intermediate)

DO all samples

$>\mathrm{R}^{*} \mathrm{dM}_{\mathrm{g}-\text { sol-s }}=-\mathrm{R}^{*} \mathrm{dM}_{\mathrm{g} \text {-dis }}$

Set sample data

Call CALIBR------>corrected $T_{s}$ \& $P_{s}$

Call ONEDIM-----> $\mathrm{T}_{\mathrm{gs}}$

Call VAPOR-------> $\mathrm{P}_{\mathrm{vs}}$

Call BB-..--.-- $>z_{s-1}$

Call BB-......-.-.-> $>\mathrm{z}_{\mathrm{s}-2}$

Call SOLUBL-----> $\mathrm{R}^{*} \mathrm{~N}_{\mathrm{g} \text {-dis-s1 }}$

Call SOLUBL--.--->R* $\mathrm{R}_{\mathrm{g} \text {-dis-s2 }}$

Calc $\mathrm{V}_{\mathrm{gs}}$ for each sample

Calc $\mathrm{R}^{*} \mathrm{dM}_{\mathrm{s}}$

Establish location of Ref Pt relative to samples

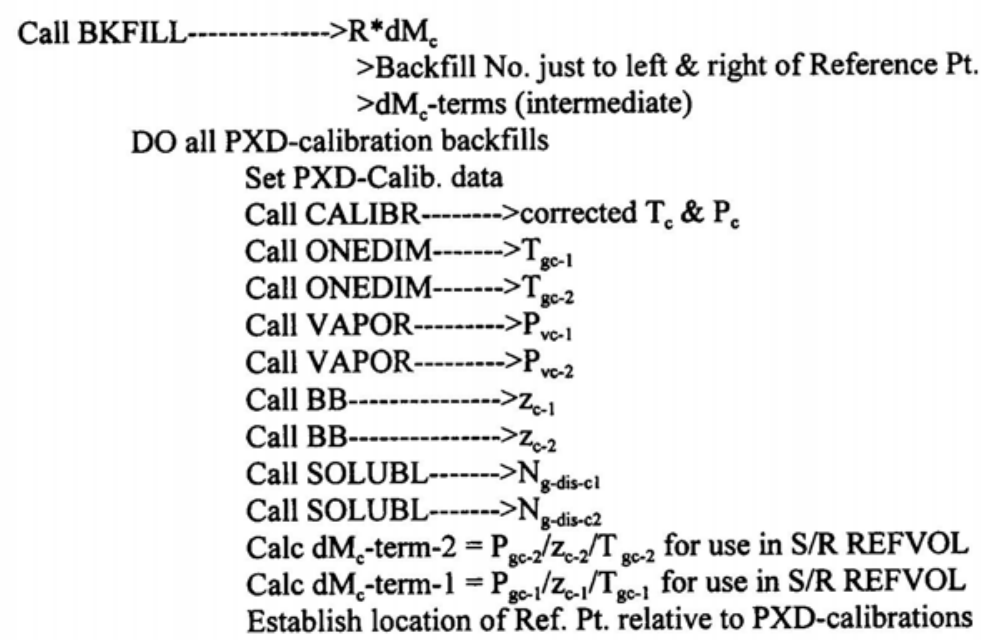

ggelwplflo-diag-7.wpd 
Begin iteration loop to adjust HS volume measurements for commonality at the

Reference Pt. conditions and in process determine Vg-ave and Beta-Lo and Beta-Hi

Input Beta data, adjusting for calibrations, HS-gas temp and -pressure and compute compressibility

DO all Betas

READ Beta-step data

Call CALIBR------>corrected $\mathrm{T}_{\mathrm{K}} \& \mathrm{P}_{\mathrm{K}}$

Call ONEDIM------> $\mathrm{T}_{\mathrm{g} K \mathrm{~K}}$

Call VAPOR-------->P $P_{v k}$

Call BB----.-----> $\mathrm{z}_{\mathrm{k}}$

READ Beta-start data

Call CALIBR------->corrected $T_{B} \& P_{B}$

Call ONEDIM--.--.->>T $\mathrm{T}_{8 \mathrm{~B}}$

Call VAPOR--------> $>P_{v B}$

Call BB----------> $\mathrm{z}_{\mathrm{B}}$

$\mathrm{DO}$ all samples $\left(\mathrm{V}_{\mathrm{g}}\right.$ measurements)

Set initial guess of $\mathrm{V}_{\mathrm{g}}$ at Ref. Pt. Conditions equal to ave of $\mathrm{V}_{\mathrm{g}}$

Begin Iteration Loop for $\mathrm{V}_{\mathrm{g} \text {-ave }}$ at the Ref. Pt. conditions by successive substitution of calculated $\mathrm{V}_{\mathrm{g} \text {-ave }}$ for $\mathrm{V}_{\mathrm{g} \text {-guess }}$

Calc $\mathrm{R}^{*} \mathrm{M}_{\mathrm{g}}{ }^{\prime}=\mathrm{P}_{\mathrm{g}}{ }^{\prime *} \mathrm{~V}_{\mathrm{g}-\text { guess }} / \mathrm{z}^{\prime} / \mathrm{T}_{\mathrm{g}}{ }^{\prime}$

DO all PXD Calibrations

Calc $\mathrm{R}^{*} \mathrm{dM}_{\mathrm{c}}$ using $\mathrm{dM}_{\mathrm{c}}$ terms from S/R BKFILL

Determine difference in HS-gas mass between Beta-Step and Beta-Start due changes in quantity of gas dissolved in brine to find true brine/waste expansion coefficient, Beta

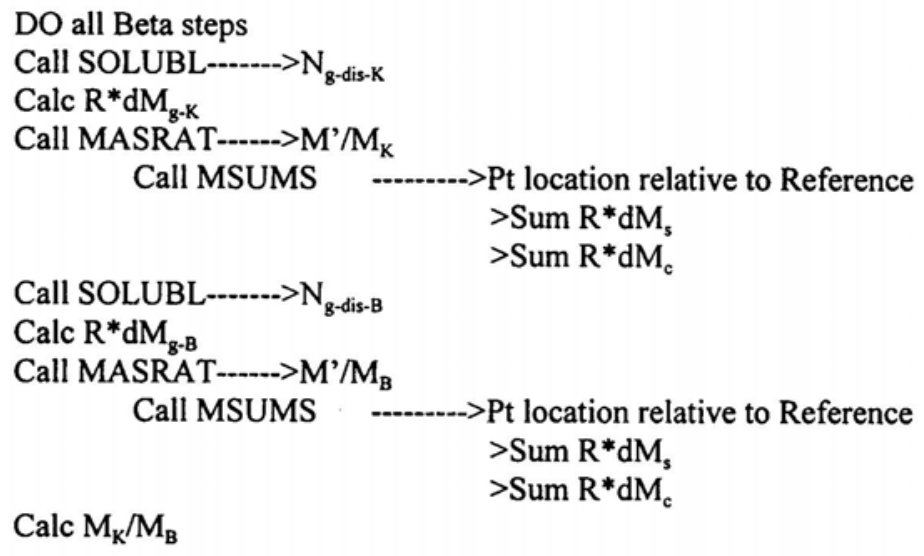

Compute Beta-Lo and Beta-Hi at the ave Beta temperatures

$\mathrm{DO}$ all samples $\left(\mathrm{V}_{\mathrm{g}}\right.$ measurements)

Compute adjusted $\mathrm{V}_{\mathrm{gs}}$ at the ref temperature

Find the arithmetic ave of all adjusted $V_{g s}$ (i.e., $V_{g s-a v e}$ )

Compare $\mathrm{V}_{\mathrm{gs} \text {-ave }}$ to $\mathrm{V}_{\mathrm{g} \text {-guess, }}$ iterate as required

ggelwptflo-diag-7.wpd 
Calc final value of HS-gas mass at the Ref. Pt., $\mathrm{R}^{*} \mathrm{dM}_{\mathrm{g}}{ }^{\prime}$

DO all PXD Calibrations

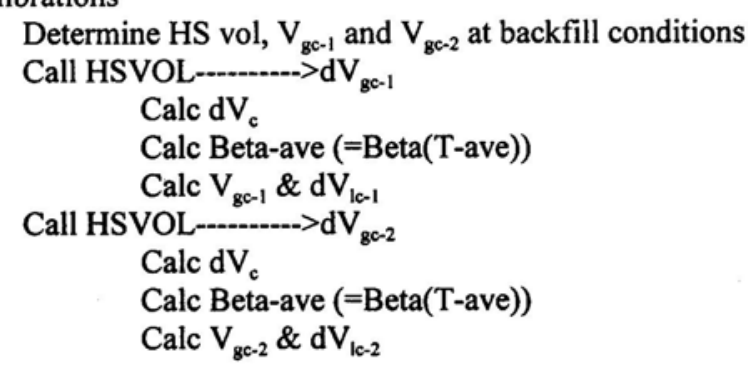

Calc $\mathrm{R}^{*} \mathrm{dM}_{\mathrm{c}}$ using $\mathrm{dM}_{\mathrm{c}}$ terms-1\&-2 from S/R BKFILL

Compute compensated pressure for all data points--input data points DO all data points

Adjust data point No. for duplicative or dropped data

Call CALIBR-----.--> corrected $T_{d} \& P_{d}$

Call ONEDIM---------> $\mathrm{T}_{\mathrm{gd}}$

Call VAPOR---------->P

Call BB--.-.-.-- $>\mathbf{z}_{d}$

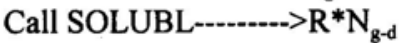

calc $\mathrm{R}^{*} \mathrm{dM}_{\mathrm{g}-\text {-sol-d }}$

Call HSVOL--.-..---->>dV

Calc $\mathrm{dV}_{\mathrm{c}}$

Calc Beta-ave (=Beta(T-ave))

Calc $V_{g d} \& d V_{l d}$

compute ratios of ref to data $\mathrm{pt}$ for $\mathrm{z}, \mathrm{T}, \mathrm{V}_{\mathrm{g}}$

Call MASRAT-

Call MSUMS -------->Pt location relative to Reference

$>$ Sum $\mathrm{R}^{*} \mathrm{dM}_{\mathrm{s}}$

$>$ Sum $R^{*} \mathrm{dM}_{\mathrm{c}}$

END

Compute adjusted pressure 


\section{ATTACHMENT 4 TO APPENDIX D}

FORTRAN Program GGE7b1 Listing

\section{PROGRAM GGE7b1}

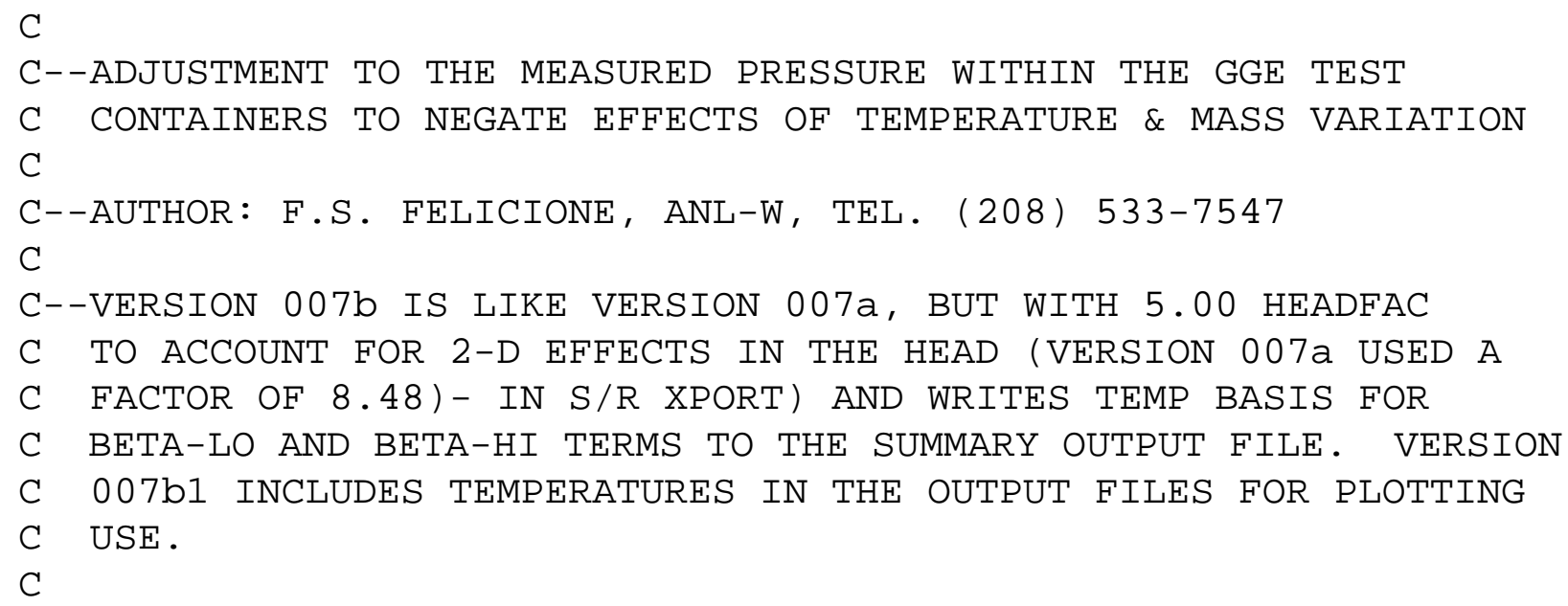


(E) ADJUSTS ALL TEMPERATURE AND PRESSURE DATA FOR ERRORS

BRINE MIXTURE FROM INDUCED TEMPERATURE STEPS

(F) TRACKS HS-GAS MASS AS IT CHANGES DUE TO GAS SAMPLE EXTRACTION AND BACKFILL ERRORS DURING PXD CALIBRATION

C--THE USER PROVIDES INPUT TO THE PROGRAM VIA THE UNFORMATTED

C TEXT FILE GGESET.TXT. THIS FILE CONTAINS ALL GEOMETRIC INFO, C MATERIAL PROPERTIES, CERTAIN CONSTANTS, CALIBRATION INFO FOR

C THE RTD'S AND PXD'S, GAS-SAMPLING DATA, REFERENCE CONDITIONS,

C INDUCED TEMPERATURE-STEP DATA, AND (OPTIONALLY) A LIST OF THE

C POINTS TO BE COMPENSATED. FOR CONVENIENCE, THE USER MAY

C CHOOSE TO PROVIDE THE LIST AS A SEPARATE TEXT FILE, GGESTR.TXT

C VERY USEFUL WHEN THE LIST IS "IMPORTED" TO THIS PROGRAM.

C

C--TWO SPACE-DELIMITED TEXT FILES ARE PRODUCED BY THE PROGRAM:

C (1) GGE.OUT - THIS FILE WRITES MIRRORS OF VIRTUALLY ALL INPUT TERMS AND INTERMEDIATE COMPUTATION VALUES, MOSTLY FOR USE IN DEBUGGING THE PROGRAM, BUT ALSO TO OBTAIN QUANTITIES SUCH AS TRANSPORT COEFFICIENTS, ETC.

(2) GGESUM.OUT - THIS FILE WRITES A VERY FEW KEY PARAMETERS AND THE LIST OF MEASURED AND TEMPERATURE-COMPENSATED PRESSURE. THIS FILE CAN BE IMPORTED INTO A PLOTTING PROGRAM (SUCH AS EXCEL) FOR GRAPHIC DEPICTION OF RESULTS

C

C

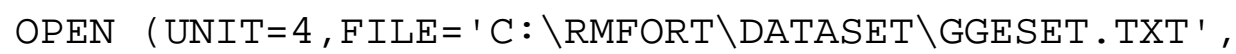

$$
\begin{aligned}
& 1 \text { STATUS= ' OLD', BLANK= 'ZERO ' ) }
\end{aligned}
$$

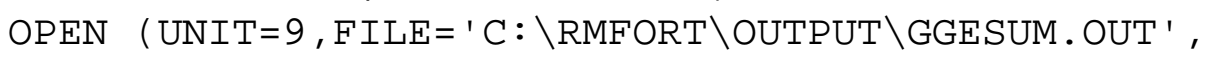

1 STATUS= ' UNKNOWN ' )

OPEN (UNIT=10, FILE= ' C : \RMFORT\OUTPUT\GGE . OUT' ',

1 STATUS= 'UNKNOWN' $)$

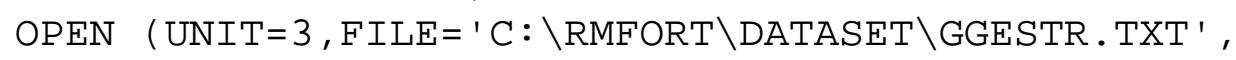

C 1 STATUS= 'OLD', BLANK= 'ZERO' )

C--ESTABLISH ALL GEOMETRIC FACTORS AND PROPERTIES IN ONEDIM CALL ONEDIM(1, DUM1, DUM2, DUM3, DUM4)

C

C--INPUT VAPOR-PRESSURE DATA INTO S/R VAPOR 


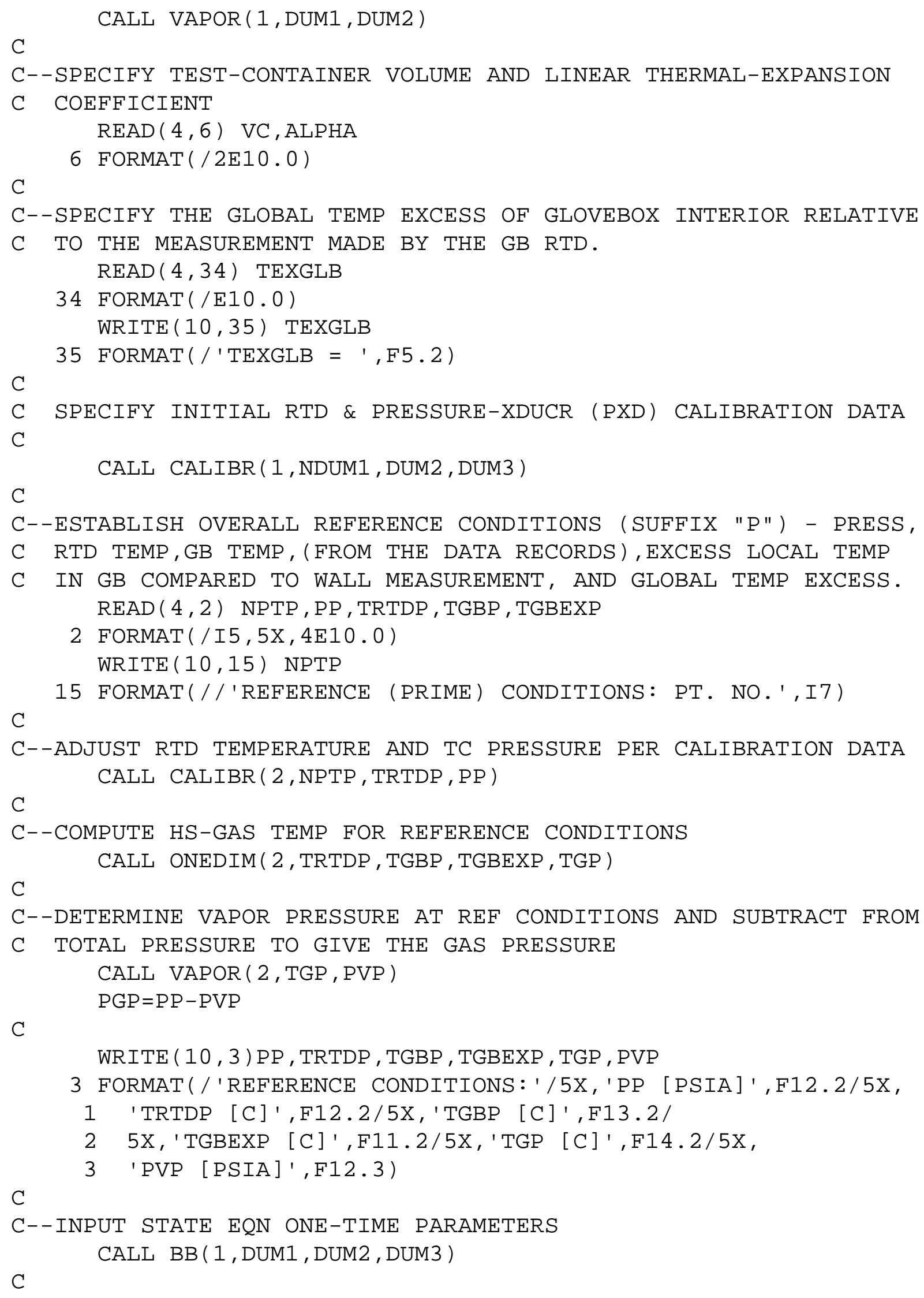




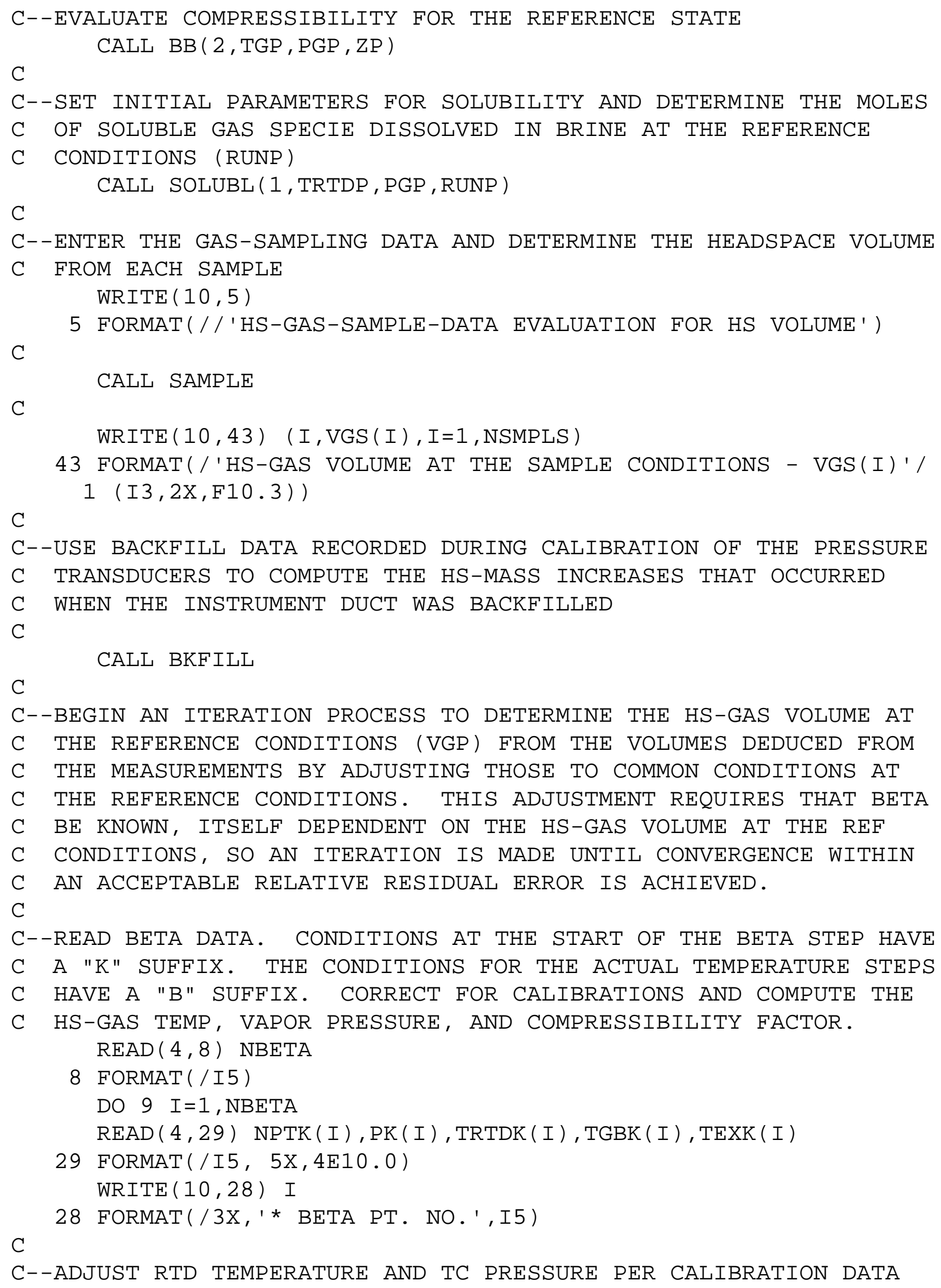




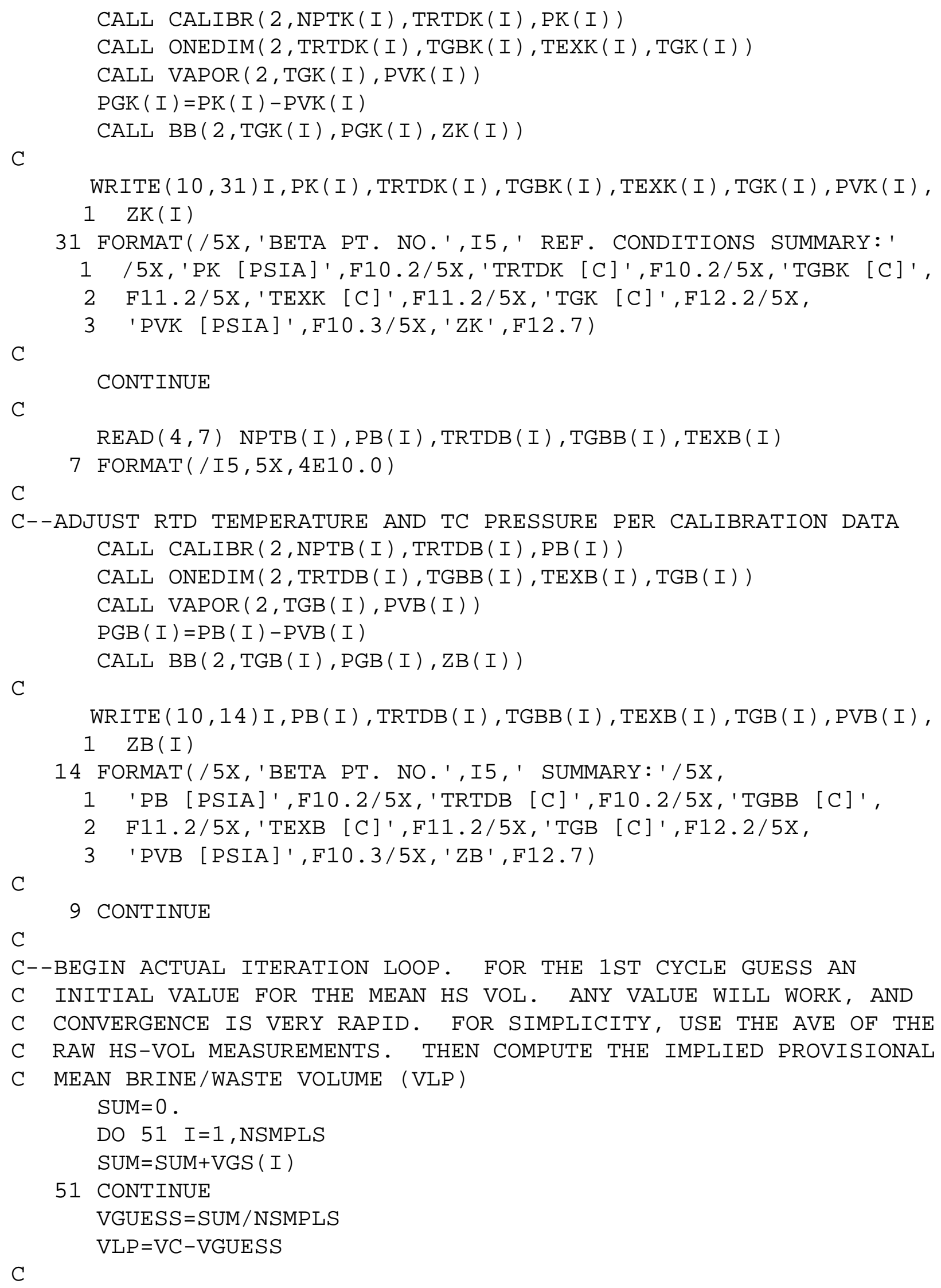

51 CONTINUE VGUESS $=$ SUM/NSMPLS VLP $=$ VC - VGUESS

C 
WRITE $(10,52)$ VGUESS, VLP

52 FORMAT(/'BEGIN ITERATION FOR MEAN HS VOLUME AND BETA'/

1 'INITIAL ASSUMPTIONS: '/10X, 'VGP=' , F10.3,10X, 'VLP=' , F10.3)

C

ICYCLE $=\odot$

$E R R=0.00001$

MAXCYC $=100$

C

38 CONTINUE

ICYCLE $=$ ICYCLE+1

IF (ICYCLE .GT. MAXCYC)STOP

C

1 'MEAN HS VOLUME CYCLE LIMIT REACHED'

C--NOW USE SUCCESSIVE SUBSTITUION TO ITERATE THE AVE HS VOLUME

C AT REF CONDITIONS UNTIL CONVERGED

IF (ICYCLE . GT . 1)VGUESS=VGOAVE

C

C--THE PRESSURE CHANGE AT THE BETA STEP MAY BE PARTIALLY DUE TO

C A CHANGE IN THE HS-GAS MASS THAT RESULTED FROM A CHANGE IN THE

C QUANTITY OF SOLUBLE GAS DISSOLVED IN THE BRINE. THIS MUST BE

C ACCOUNTED FOR TO YIELD A BETA VALUE THAT REFLECTS ONLY THE

C THE EXPANSION/CONTRACTION CHARACTERISTICS OF THE BRINE/WASTE.

C FOR PROGRAMMING CONVENIENCE, THE RATIO OF HS-GAS MASS BEFORE

C AND AFTER THE BETA STEP ARE FOUND BY COMBINING RATIOS RELATIVE

C TO THE OVERALL REF PT CONDITIONS.

$\mathrm{C}$

C FIRST COMPUTE HS-GAS MASS ( ${ }^{*}$ GAS CONSTANT) AT THE REF

C CONDITIONS AND CURRENT ESTIMATE OF HS VOLUME RMGP $=P G{ }^{*} V G U E S S / Z P /(273.15+T G P)$

C

DO $58 \mathrm{I}=1$, NBETA

$\operatorname{CALL} \operatorname{SOLUBL}(2, \operatorname{TRTDK}(\mathrm{I}), \operatorname{PGK}(\mathrm{I}), \mathrm{RUNK})$

RDMDIS $=$ RUNK - RUNP

RDMGK $=-$ RDMDIS

C

CALL MASRAT(NPTK(I), RDMGK, WRATK)

$\operatorname{CALL} \operatorname{SOLUBL}(2, \operatorname{TRTDB}(\mathrm{I}), \operatorname{PGB}(\mathrm{I}), \mathrm{RUNB})$

RDMDIS=RUNB - RUNP

RDMGB $=-$ RDMDIS

CALL MASRAT(NPTB (I ), RDMGB, WRATB)

C

WBETA $(I)=W R A T K /$ WRATB

58 CONTINUE

$\mathrm{C}$

C COMPUTE BETA-LO (BETA(1)) AND BETA-HI (BETA(2))AT THE AVE

C TEMPERATURES OF THE RESPECTIVE BETA STEPS BASED ON THE CURRENT 
C VGUESS (NOTE: NBETA=2 ALWAYS, IN THE PRESENT FORMULATION) DO $53 \mathrm{I}=1$, NBETA

$\operatorname{TBETA}(I)=(\operatorname{TRTDB}(\mathrm{I})+\operatorname{TRTDK}(\mathrm{I})) / 2$.

$\operatorname{DTSTEP}=\operatorname{TRTDB}(\mathrm{I})-\operatorname{TRTDK}(\mathrm{I})$

$D V C=3 .{ }^{*} A L P H A * V C * D T S T E P$

PRATIO $=$ PGK $(I) / P G B(I)$

ZRATIO $=Z K(I) / Z B(I)$

TRATIO $=($ TGK $(I)+273.15) /(T G B(I)+273.15)$

BRACK $=$ PRATIO *WBETA $(\mathrm{I}) /$ ZRATIO/TRATIO -1 .

BRACE $=1 .+($ DVC - VGUESS *BRACK $) /$ VLP

$\operatorname{BETA}(\mathrm{I})=1 . / \mathrm{DTSTEP} *$ ALOG $($ BRACE $)$

C

WRITE $(10,12)$ I , ZRATI0, TRATI0, PRATI0, DTSTEP, TBETA( I) , DVC,

1 BRACK, BRACE, BETA(I)

12 FORMAT(/5X, 'PARAMETERS FOR BETA PT. NO. ', I3

1 /5X, 'ZRATIO' , F12.7/5X, 'TRATI0' ,F12.7/5X, 'PRATIO',

2 F12.7/5X, 'DTSTEP' , 6X, F5.2/5X, 'TBETA(I) ', 4X, F5.2/5X, 'DVC',

3 E18.6/5X, 'BRACK' ,E16.6/5X, 'BRACE' ,E16.6/5X, 'BETA(I) ',

4 E14.6)

C

53 CONTINUE

C

C--NOW COMPUTE THE TEMPERATURE-DEPENDENT VALUE OF BETA (BETAI)

C APPLICABLE TO EACH VOLUME MEASUREMENT BY LINEARLY

C INTERPOLATING BETWEEN BETA-LO (IE, BETA(1)) AND BETA-HI

C (IE, BETA(2)) FROM ABOVE, BASED ON THE AVE TEMPERATURE (TIAVE)

C BETWEEN THE MEASUREMENTS AND REF RTD TEMPERATURES.

C

C THEN COMPUTE THE ADJUSTED HS VOLUME FOR EACH MEASUREMENT WHEN

C ADJUSTED TO THE REF TEMPERATURE (VGOI) AND FIND THE AVE OF

C THESE (VGOAVE)

SUM $=\odot$.

DO $54 \mathrm{I}=1$, NSMPLS

TIAVE $=(\operatorname{TRTDS}(\mathrm{I})+\operatorname{TRTDP}) / 2$.

BBARI $=\operatorname{BETA}(1)+(\operatorname{BETA}(2)-\operatorname{BETA}(1)) *(\operatorname{TIAVE}-\operatorname{TBETA}(1)) /$

1 ( TBETA(2) - TBETA(1))

DTSI $=$ TRTDS $(\mathrm{I})-$ TRTDP

DVCI $=3 .{ }^{*} A L P H A * V C * D T S I$

$V G O I=(V G S(I)+V C *(E X P(B B A R I * D T S I)-1)-.D V C I) / E X P(B B A R I * D T S I)$ SUM $=S U M+V G O I$

54 CONTINUE

VGOAVE $=$ SUM/NSMPLS

$\mathrm{C}$

C--COMPARE THE CALCULATED AVE HS VOLUME (VGOAVE) TO THE ASSUMED

C VALUE (VGUESS) AND ITERATE UNTIL THE RELATIVE ERROR IS WITHIN

C THE USER-SPECIFIED INTERVAL (ERR) 
C

RELERR= (VGOAVE - VGUESS $) /$ VGUESS

WRITE $(10,41)$ ICYCLE, VGUESS, VG@AVE, RELERR, BETA(1), $\operatorname{BETA}(2)$

41 FORMAT(/'MEAN HS VOLUME DETERMINATION CYCLE NO. ' , I5

1 /10X, 'VGUESS= ', F10.3/10X, 'VGOAVE= ', F10.3

2 /10X, 'RELERR= ', F12.6/10X, 'BETA(1)=' , E15.4

$3 / 10 X,{ }^{\prime} \operatorname{BETA}(2)=$ ', E15.4)

IF (ABS(RELERR) . GT. ERR)GO TO 38

C

C--CALCULATE BRINE/WASTE VOLUME AT THE O.A. REFERENCE CONDITIONS

VGP $=$ VGOAVE

VLP $=V C-V G P$

WRITE $(10,11) \operatorname{VGP}, \operatorname{VLP}$

11 FORMAT( /'FINAL REF VALUES'/5X, 'VGP',F15.3/5X, 'VLP', F14.2)

C

C--COMPUTE FINAL HS-GAS MASS*GAS CONSTANT AT THE REF CONDITIONS $\mathrm{RMGP}=\mathrm{PGP}{ }^{*} \mathrm{VGP} / \mathrm{ZP} /(273 \cdot 15+\mathrm{TGP})$

$\mathrm{C}$

C--CALCULATE THE HS VOLUMES AT THE BACKFILL CONDITIONS DO $57 \mathrm{I}=1$, NCALP

CALL HSVOL(TRTDC1(I), DVGC1 (I ), DUM)

CALL HSVOL(TRTDC2(I), DVGC2(I), DUM)

57 CONTINUE

C

C--CALCULATE THE HS-GAS MASS (ACTUALLY GAS CONSTANT*MASS, R*DM)

C INCREASE DURING EACH BACKFILL BASED ON THE ACTUAL HS VOLUME

C AT THE BACKFILL CONDITIONS

DO $55 \mathrm{I}=1$, NCALP

VC1=VGP+DVGC1 ( I )

VC2=VGP+DVGC2(I)

$\operatorname{RDMC}(\mathrm{I})=\mathrm{VC} 2{ }^{*} \mathrm{DMCT} 2(\mathrm{I})-\mathrm{VC} 1{ }^{*} \mathrm{DMCT} 1$ ( I )

55 CONTINUE

C

WRITE $(10,56)$ RMGP, ( I, RDMC ( I ), I=1, NCALP )

56 FORMAT ( / HS-GAS MASS*R=', E15.8/

1 'BACKFILL HS-GAS-MASS INCREASE (R*DMC) '/10X, ' I', 5X,

2 'RDMC ( I ) '/(9X, I2, 5X, E12.6))

C

C--GENERATE A SUMMARY FILE OF RESULTS FOR HS-GAS VOLUME, BETA,

C DATA-POINT NO., DATE\&HR, MEASURED PRESSURE, FINAL PRESSURE (AS

C ADJUSTED) AND HS-GAS MASS

C

$\operatorname{WRITE}(9,33) \operatorname{VgP}, \operatorname{tBetA}(1), \operatorname{BETA}(1), \operatorname{TBETA}(2), \operatorname{BETA}(2), \operatorname{TEXgLB}$,

1 ICYCLE

33 FORMAT( 'VGP: ' , 10X, E12.5/'TBETA(1): ' ,7X, E12.4/

1 'Beta(1): ' , 7X, E12.4/'Tbeta(2): ' ,7X, E12.4/'BetA(2): ', 


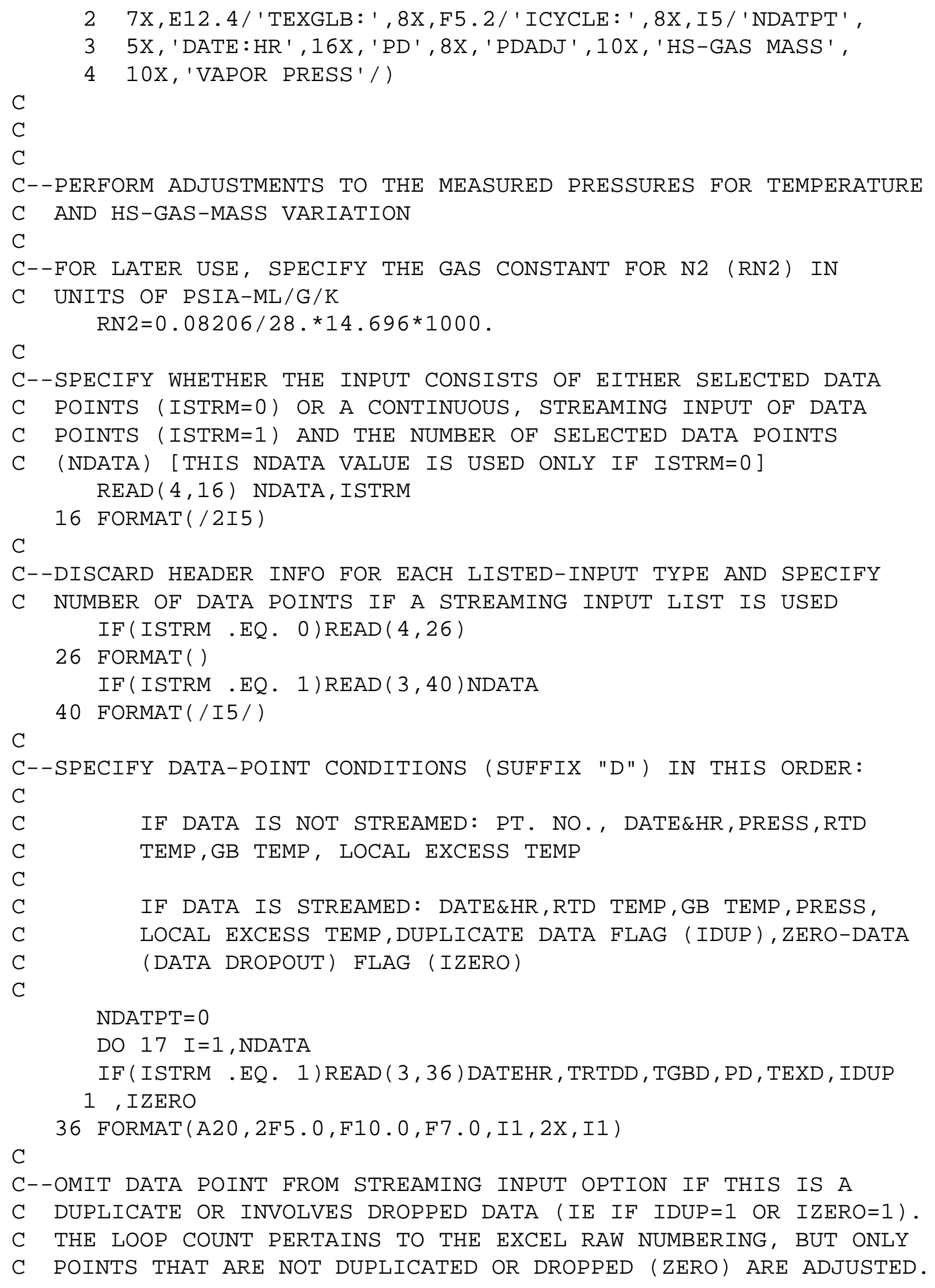


C THE EXCEL RAW DATA POINT NO. IS PRESERVED IN THE OUTPUT FILE,

C BUT ALL SAMPLES, CALIBRATIONS, REFERENCE POINTS, BETA POINTS,

C ETC REFER TO THE POINT NO. WHEN ADJUSTED FOR DUPLICATE AND

C DROPPED (ZERO) DATA.

C

IF(ISTRM .EQ. 1 .AND. IDUP .EQ. 1)GO TO 17

IF(ISTRM .EQ. 1 .AND. IZERO .EQ. 1)GO TO 17

NDATPT $=$ NDATPT +1

$\operatorname{IF}($ ISTRM . EQ . $\odot)$ READ $(4,18)$ NDATPT, DATEHR, PD, TRTDD, TGBD, TEXD

18 FORMAT (I5, A20, 4E10.๑)

$\mathrm{C}$

C--DETERMINE THE DATA POINT HS-GAS TEMP, VAPOR PRESS, AND

C COMPRESSIBILITY FACTOR BY CALLING S/R ONEDIM, S/R VAPOR, AND

C S/R BB, AS WAS DONE FOR THE OTHER POINTS ABOVE.

C EVALUATE BETA AT THE AVERAGE TEMP BETWEEN REF AND DATA.

C THEN DETERMINE THE TEST-CONTAINER EXPANSION AND THE

C WASTE/BRINE EXPANSION RELATIVE TO THE REFERENCE CONDITIONS.

C FINALLY, COMPUTE THE REF/DATA RATIOS FOR Z, TG, AND VG. THE

C TEMP-ADJUSTED PRESSURE IS PGD*ZRATIO*TRATIO/VRATIO*WRATIO.

C

C--THE CURRENT CONSTRUCT ASSUMES TWO BETA VALUES ONLY: BETA(1),

C A LOW-TEMP STEP VALUE AT TEMP TBETA(1) AND BETA(2), A HIGH-

C TEMP STEP VALUE AT TEMP TBETA(2). THE BETA VALUE FOR USE IN

C THE PRESS ADJUSTMENT IS BETBAR (BETA-BAR, IE AVE) DETERMINED

C BY INTERPOLATING LINEARLY BETWEEN BETA(1) AND BETA(2) AT THE

C AVE TC RTD TEMP.

$\mathrm{C}$

$\operatorname{WRITE}(10,19)$ NDATPT, DATEHR, PD, TRTDD, TGBD, TEXD

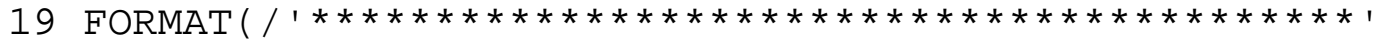

$1 / 1 * * *$ NEW DATA POINT $* * * * * * * * * * * * * * * * * * * * * * * * 1$

2 /5X, 'NDATPT', I9/5X, 'DATE: HR' , A20/5X, 'PD', F13.2/5X,

3 'TRTDD', F10.2/5X, 'TGBD', F11.2/5X, 'TEXD', F11.2)

C

C--ADJUST RTD TEMPERATURE PER CALIBRATION DATA

$\operatorname{CALL} \operatorname{CALIBR}(2, \operatorname{NDATPT}, \operatorname{TRTDD}, \mathrm{PD})$

C

CALL ONEDIM(2, TRTDD, TGBD, TEXD, TGD)

CALL VAPOR $(2, T G D, P V D)$

$\operatorname{WRITE}(10,27)$ TGD

27 FORMAT(/5X, 'HS-GAS TEMP, TGD, FROM ONEDIM: ',F8.3)

$P G D=P D-P V D$

$\mathrm{C}$

CALL BB $(2, T G D, P G D, Z D)$

C--DETERMINE THE MOLES OF SOLUBLE GAS SPECIE DISSOLVED IN BRINE

C AT THE DATA-POINT CONDITIONS TIMES UNIV GAS CONSTANT (RUND)

CALL SOLUBL (2, TRTDD, PGD, RUND) 


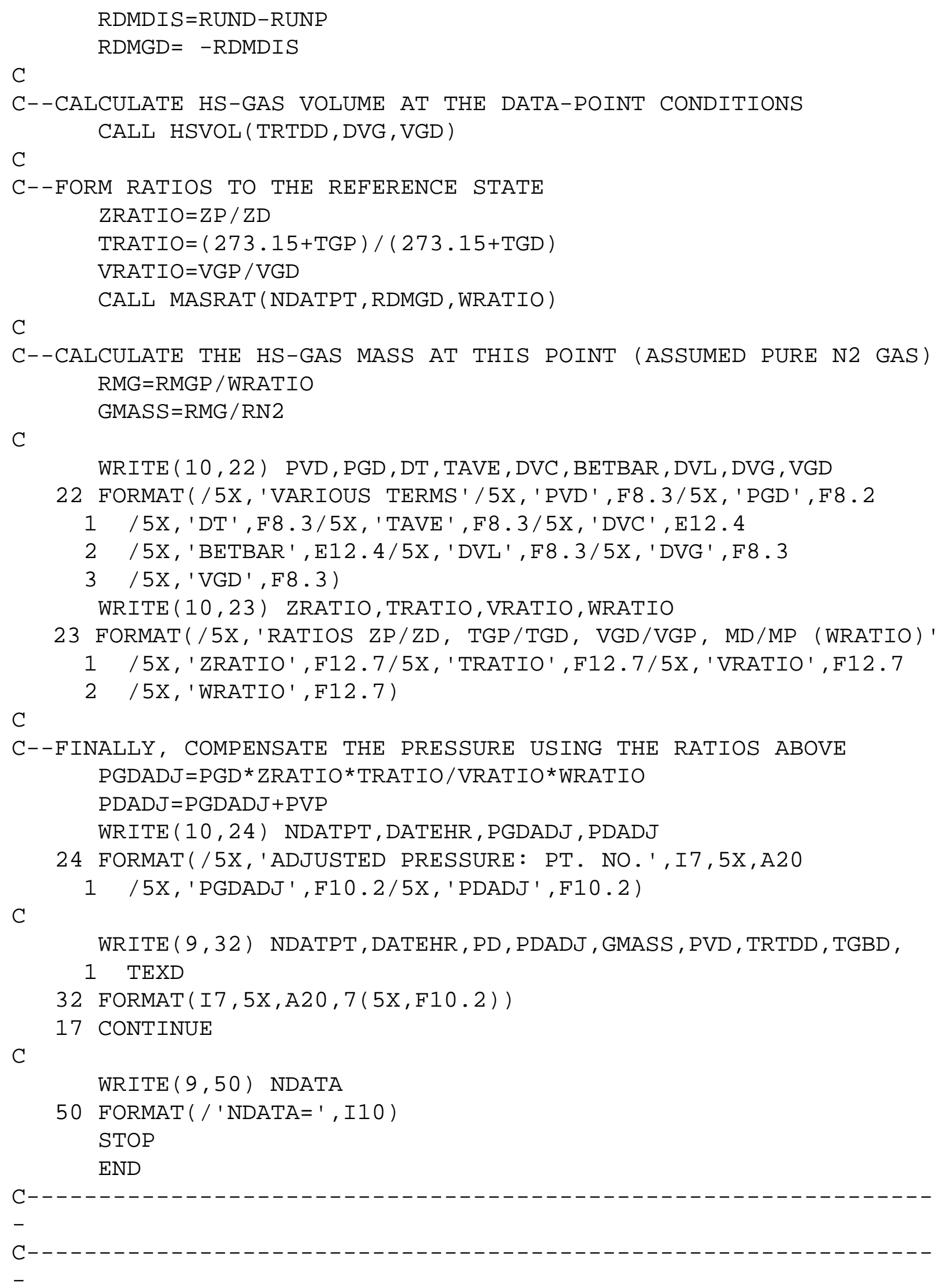




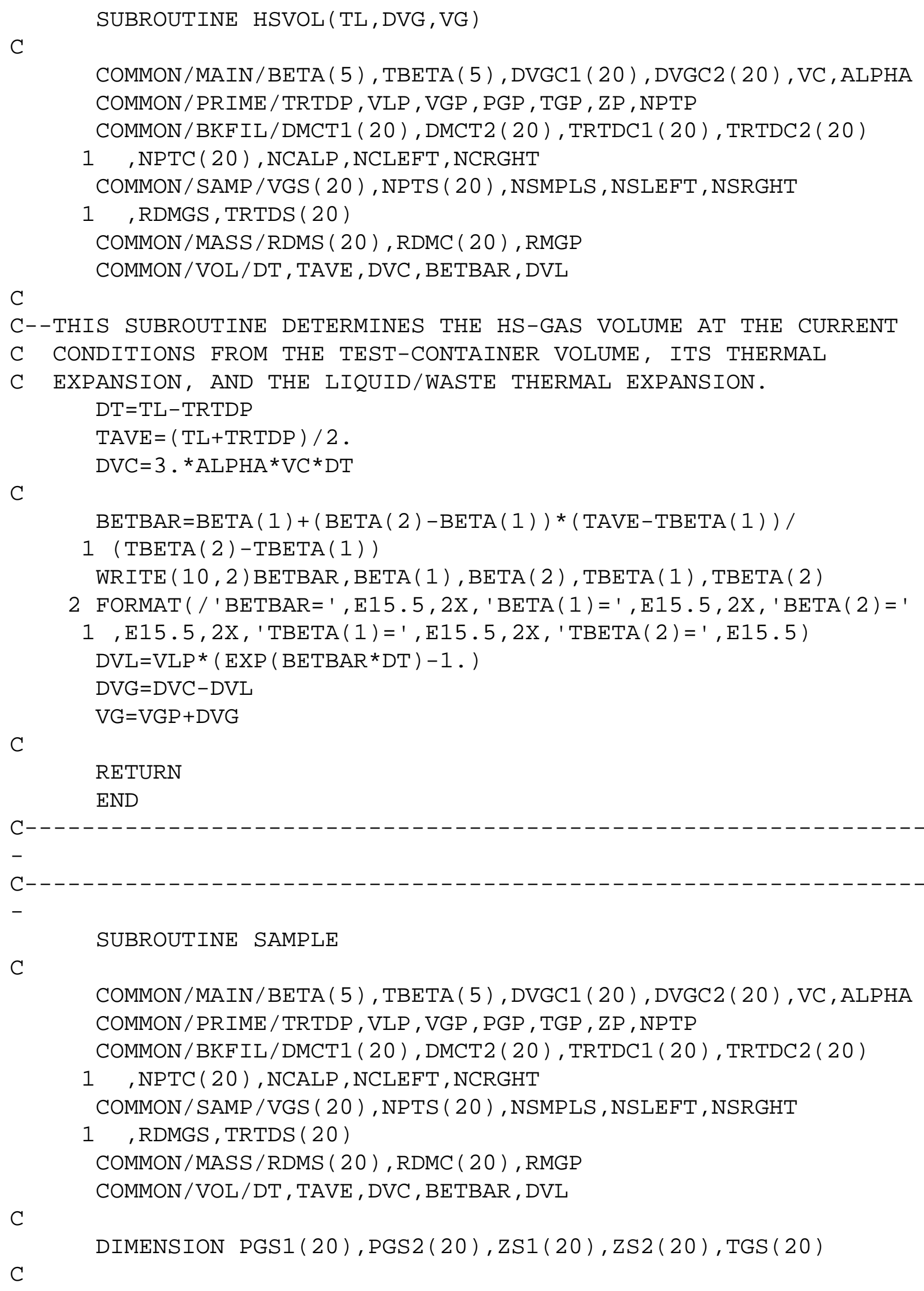


C--THIS SUBROUTINE TREATS THE DATA GATHERED DURING SAMPLING OF

C THE HS GAS. THE HS VOLUME (AT THE SAMPLE CONDITIONS) IS

C DEDUCED, THE MASS OF GAS EXTRACTED IS DETERMINED, AND THE

C CHRONOLOGY OF SAMPLES RELATIVE TO THE USER-SELECTED REFERENCE

C POINT IS DETERMINED

C

C--INPUT SAMPLE MEASUREMENTS: SAMPLE VOLUME IS VSAMPL; THE NUMBER

C OF SAMPLE POINTS IS NSMPLS $\operatorname{READ}(4,1)$ VSAMPL

$1 \operatorname{FORMAT}(/ \mathrm{E} 10.0)$

$\operatorname{READ}(4,4)$ NSMPLS

4 FORMAT(/I5)

IF(NSMPLS .EQ. $\odot)$ STOP'NO HS-GAS SAMPLE INPUT PROVIDED'

$\mathrm{C}$

C--INPUT GAS-SAMPLE DATA AND COMPUTE PARAMETERS FOR EACH OF THE

C NSMPLS TOTAL SAMPLE PTS

$\operatorname{READ}(4,11)$

C

11 FORMAT( )

DO $3 I=1, N S M P L S$

$\operatorname{WRITE}(10,15) \mathrm{I}$

15 FORMAT(/'SAMPLE NO. ', I4, ' POINT EVALUATION')

$\operatorname{READ}(4,2) \operatorname{NPTS}(\mathrm{I}), \mathrm{PS} 1, \mathrm{PS} 2, \operatorname{TRTDS}(\mathrm{I}), \operatorname{TGBS}, \operatorname{TGBEXS}$

$\mathrm{C}$

2 FORMAT $(3 X, 1 X, I 5,1 X, 5 E 10.0)$

C--ADJUST RTD TEMPERATURE \& TC PRESSURE PER CALIBRATION DATA

C (PS1 \& PS2 ARE ADJUSTED SIMULTANEOUSLY)

$\mathrm{PS}=\mathrm{PS} 1$

$\operatorname{CALL} \operatorname{CALIBR}(2, \operatorname{NPTS}(\mathrm{I}), \operatorname{TRTDS}(\mathrm{I}), \mathrm{PS})$

$A D J=P S-P S 1$

$P S 1=P S 1+A D J$

$P S 2=P S 2+A D J$

C

C--COMPUTE THE HEADSPACE GAS TEMPERATURE FOR THIS SAMPLE DATA $\operatorname{CALL} \operatorname{ONEDIM}(2, \operatorname{TRTDS}$ (I), TGBS, TGBEXS, TGS (I))

$C$

C--DETERMINE WATER VAPOR PRESSURE FOR THIS SAMPLE DATA AND

C SUBTRACT FROM THE TOTAL PRESSURE TO GIVE SAMPLE GAS PRESSURE $\operatorname{CALL} \operatorname{VAPOR}(2, \mathrm{TGS}(\mathrm{I}), \mathrm{PVS})$

PGS1 ( I ) =PS1 -PVS

$\mathrm{C}$ PGS2 ( I ) =PS2 - PVS

C--DETERMINE THE COMPRESSIBILITY FACTORS ZS1 \& ZS2

$\operatorname{CALL~BB}(2, \operatorname{TGS}(\mathrm{I}), \operatorname{PGS1}(\mathrm{I}), \mathrm{ZS} 1(\mathrm{I}))$

$\operatorname{CALL~BB}(2, \operatorname{TGS}(\mathrm{I}), \operatorname{PGS} 2(\mathrm{I}), \mathrm{ZS} 2(\mathrm{I}))$

C 
C--DETERMINE THE MOLES OF SOLUBLE GAS SPECIE DISSOLVED IN BRINE

C FOR THESE SAMPLE CONDITIONS (RUNS1 AND RUNS2) AND THE CHANGES

C IN HS GAS MASS TIMES R (RDMGS)

CALL SOLUBL (2, TRTDS (I), PGS1 (I), RUNS1)

CALL SOLUBL ( $2, \operatorname{TRTDS}$ (I), PGS2 (I), RUNS2)

RDMDIS $=$ RUNS2 - RUNS1

RDMGS $=-$ RDMDIS

C

C--CALCULATE THE HEADSPACE-GAS VOLUME FOR THIS MEASUREMENT

TRM1=PGS1 ( I )/ZS1 ( I)

TRM2=PGS2 (I)/ZS2 (I)

C $\operatorname{VGS}(\mathrm{I})=\left(\right.$ TRM2 ${ }^{*}$ VSAMPL - RDMGS $^{*}($ TGS $\left.(\mathrm{I})+273.15)\right) /($ TRM1 - TRM2 $)$

C--CALCULATE THE HS-GAS MASS (ACTUALLY GAS CONSTANT*MASS, R*DMS)

C ADDED (NEGATIVE OF AMT. EXTRACTED) DURING THIS GAS SAMPLE $\operatorname{RDMS}(\mathrm{I})=-\mathrm{TRM} 2 *$ VSAMPL $/(273.15+\mathrm{TGS}(\mathrm{I}))$

C

$\operatorname{WRITE}(10,6) \quad I, \operatorname{TGS}(\mathrm{I}), \mathrm{PVS}, \mathrm{ZS1}(\mathrm{I}), \mathrm{ZS} 2(\mathrm{I}), \operatorname{VGS}(\mathrm{I}), \operatorname{RDMS}(\mathrm{I})$

6 FORMAT(/5X, 'SAMPLE NO. ', I3, $1 \mathrm{X}$, ' SUMMARY' $/ 10 \mathrm{X}$, 'TGS $=$ ' , F6.2/

1 10X, 'PVS =', F7.3/10X, 'ZS1 =', F10.7/10X, 'ZS2 =' ,F10.7/

C

$210 \mathrm{X}$, 'VGS $=$ ', F8.2/10X, 'RDMS =', E12.6)

3 CONTINUE

C

$\operatorname{WRITE}(10,7)(\mathrm{I}, \operatorname{RDMS}(\mathrm{I}), \mathrm{I}=1, \operatorname{NSMPLS})$

7 FORMAT(/'SAMPLE MASS EXTRACTION ( $R^{*}$ DMS $)$ ' /

$\mathrm{C}$

1 10X, ' I' , 5X, 'RDMS( I )' / (9X, I2 , 5X, E12.6) )

C--DETERMINE THE NUMBER OF THE LAST SAMPLE POINT (NSLEFT) JUST

C PRIOR TO (IE, TO THE LEFT OF) THE O.A. REFERENCE POINT; NSRGHT

C IS THE FIRST SAMPLE POINT AFTER (IE TO THE RIGHT OF) THE O.A.

C REF POINT

DO $44 \mathrm{I}=1$, NSMPLS

NSLEFT $=\mathrm{I}$

IF(NPTS(I) . GE. NPTP)GO TO 45

44 CONTINUE

GO TO 50

45 NSLEFT $=\mathrm{I}-1$

50 CONTINUE

NSRGHT $=$ NSLEFT +1

WRITE $(10,20)$ NSLEFT, NSRGHT

C

$20 \operatorname{FORMAT}\left(/ 5 \mathrm{X},{ }^{\prime} \mathrm{NSLEFT}=', \mathrm{I} 3,5 \mathrm{X},{ }^{\mathrm{NSRRGHT}}=1, \mathrm{I} 3\right)$

RETURN

END 


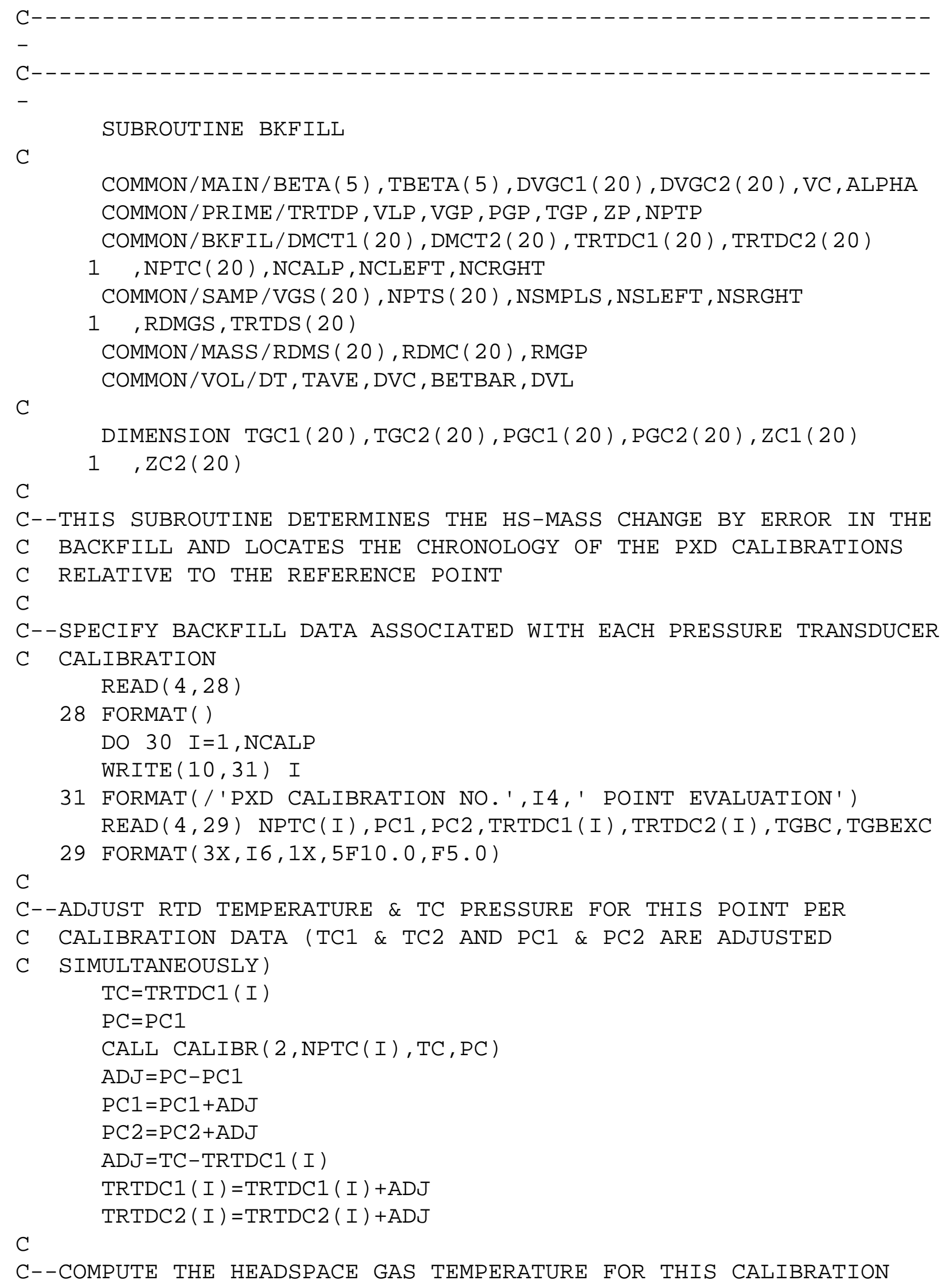


C DATA

$\operatorname{CALL} \operatorname{ONEDIM}(2, \operatorname{TRTDC} 1$ (I), TGBC, TGBEXC, TGC1(I))

$\operatorname{CALL} \operatorname{ONEDIM}(2, \operatorname{TRTDC} 2(\mathrm{I}), \operatorname{TGBC}, \operatorname{TGBEXC}, \operatorname{TGC} 2(\mathrm{I}))$

C

C--DETERMINE WATER VAPOR PRESSURE FOR THIS CALIBRATION DATA AND

C SUBTRACT FROM THE TOTAL PRESSURE TO GIVE SAMPLE GAS PRESSURE $\operatorname{CALL} \operatorname{VAPOR}(2, \operatorname{TGC} 1(\mathrm{I}), \mathrm{PVC})$

$P G C 1(I)=P C 1-P V C$

$\operatorname{CALL} \operatorname{VAPOR}(2, \operatorname{TGC} 2(\mathrm{I}), \mathrm{PVC})$

$P G C 2(I)=P C 2-P V C$

$\mathrm{C}$

C--DETERMINE THE COMPRESSIBILITY FACTORS ZC1 \& ZC2

$\operatorname{CALL~BB}(2, \operatorname{TGC} 1(\mathrm{I}), \operatorname{PGC1}(\mathrm{I}), \mathrm{ZC} 1(\mathrm{I}))$

$\operatorname{CALL} \mathrm{BB}(2, \operatorname{TGC} 2(\mathrm{I}), \operatorname{PGC} 2(\mathrm{I}), \mathrm{ZC} 2(\mathrm{I}))$

C

C--DETERMINE THE MOLES OF SOLUBLE GAS SPECIE DISSOLVED IN BRINE

C AT THESE BACKFILL CONDITIONS (ANC1 \& ANC2)

CALL SOLUBL (2, TRTDC1, PGC1 (I), ANC1)

CALL SOLUBL (2, TRTDC2, PGC1(I), ANC1)

C

$\operatorname{WRITE}(10,32) \mathrm{I}, \operatorname{TGC1}(\mathrm{I}), \operatorname{TGC} 2$ ( I), PVC, ZC1( I), ZC2 ( I)

32 FORMAT(/5X, 'PXD CAL NO. ', I3, 1X, 'SUMMARY'/10X, 'TGC1 =', F6.2

$1,10 \mathrm{X}$, 'TGC2=' ,F6.2/10X, 'PVC =' ,F7.3/10X, 'ZC1 =' ,F10.7/

$210 X, ' Z C 2=', F 10.7$ )

C

C--SAVE THE R*DMC/VOL TERM FOR USE IN S/R REFVOL

$\mathrm{DMCT} 2(\mathrm{I})=\mathrm{PGC} 2(\mathrm{I}) / \mathrm{ZC} 2(\mathrm{I}) /(273.15+\mathrm{TGC} 2(\mathrm{I}))$

C

$\operatorname{DMCT1}(\mathrm{I})=\mathrm{PGC} 1(\mathrm{I}) / \mathrm{ZC} 1(\mathrm{I}) /(273 \cdot 15+\mathrm{TGC} 1(\mathrm{I}))$

$3 \odot$ CONTINUE

C

C--DETERMINE THE NUMBER OF THE LAST PRESSURE TRANSDUCER

C CALIBRATION POINT (NCLEFT) JUST PRIOR TO (IE, TO THE LEFT OF)

C THE O.A. REFERENCE POINT; NCRGHT IS THE FIRST CALIBRATION

C POINT AFTER (IE TO THE RIGHT OF) THE O.A. REF POINT

DO $22 \mathrm{I}=1$, NCALP

NCLEFT $=\mathrm{I}$

$\operatorname{IF}(\operatorname{NPTC}(\mathrm{I})$. GE. NPTP)GO TO 21

22 CONTINUE

GO TO 23

21 NCLEFT $=\mathrm{I}-1$

23 CONTINUE

NCRGHT $=$ NCLEFT +1

WRITE $(10,24)$ NCLEFT, NCRGHT

$24 \operatorname{FORMAT}\left(/ 5 \mathrm{X},{ }^{\prime} \mathrm{NCLEFT}=1, \mathrm{I} 3,5 \mathrm{X},{ }^{\mathrm{N}} \mathrm{NCRGHT}=1, \mathrm{I} 3\right)$

C 
RETURN

END

C

SUBROUTINE MASRAT(NPT, RDMX, WRATIO)

C

COMMON/MAIN/BETA( 5 ), TBETA( 5), DVGC1(20), DVGC2(20), VC, ALPHA COMMON/PRIME/TRTDP, VLP, VGP, PGP, TGP, ZP, NPTP

COMMON/BKFIL/DMCT1(20), DMCT2(20), $\operatorname{TRTDC1}(20), \operatorname{TRTDC} 2(20)$

1 , NPTC (20) , NCALP, NCLEFT, NCRGHT COMMON/SAMP/VGS(20), NPTS(20), NSMPLS, NSLEFT, NSRGHT

1, RDMGS, TRTDS(20)

COMMON/MASS/RDMS (20), RDMC (20), RMGP

COMMON/VOL/DT, TAVE, DVC, BETBAR, DVL

C

C--THIS SUBROUTINE DETERMINES THE RATIO OF THE HS-GAS MASS

C AT THE REFERENCE POINT TO THE HS-GAS MASS AT THE POINT UNDER

C CONSIDERATION (THE VARIATION IS DUE TO SAMPLING AND BACKFILLS)

CALL MSUMS(NPT, SUMS, SUMC, NPTLOC)

GO TO $(10,20,30)$, NPTLOC

C

10 CONTINUE

C

C--POINT IS PRIOR TO REFERENCE POINT

WRATIO=RMGP / ( RMGP - SUMS - SUMC+RDMX)

GO TO 11

C

$2 \odot$ CONTINUE

C

C--POINT COINCIDES WITH THE REFERENCE POINT

WRATIO $=1.0$

GO TO 11

C

30 CONTINUE

C

C--POINT IS PAST THE REFERENCE POINT

WRATIO=RMGP $/($ RMGP+SUMS+SUMC+RDMX)

C

11 CONTINUE

WRITE $(10,12)$ NPT, SUMS, SUMC, RDMX, WRATIO

12 FORMAT( $/ 5 X$, 'MASRAT CALCULATIONS: ' /5X, 'NPT= ' , I8/5X, ' SUMS= ',

$1 \mathrm{E} 15.5 / 5 X,{ }^{\prime}$ SUMC $=$ ' , E15.5/5X, 'RDMX=' ,E15.5/5X, 'WRATIO=',

C

2 E15.5) 
RETURN

END

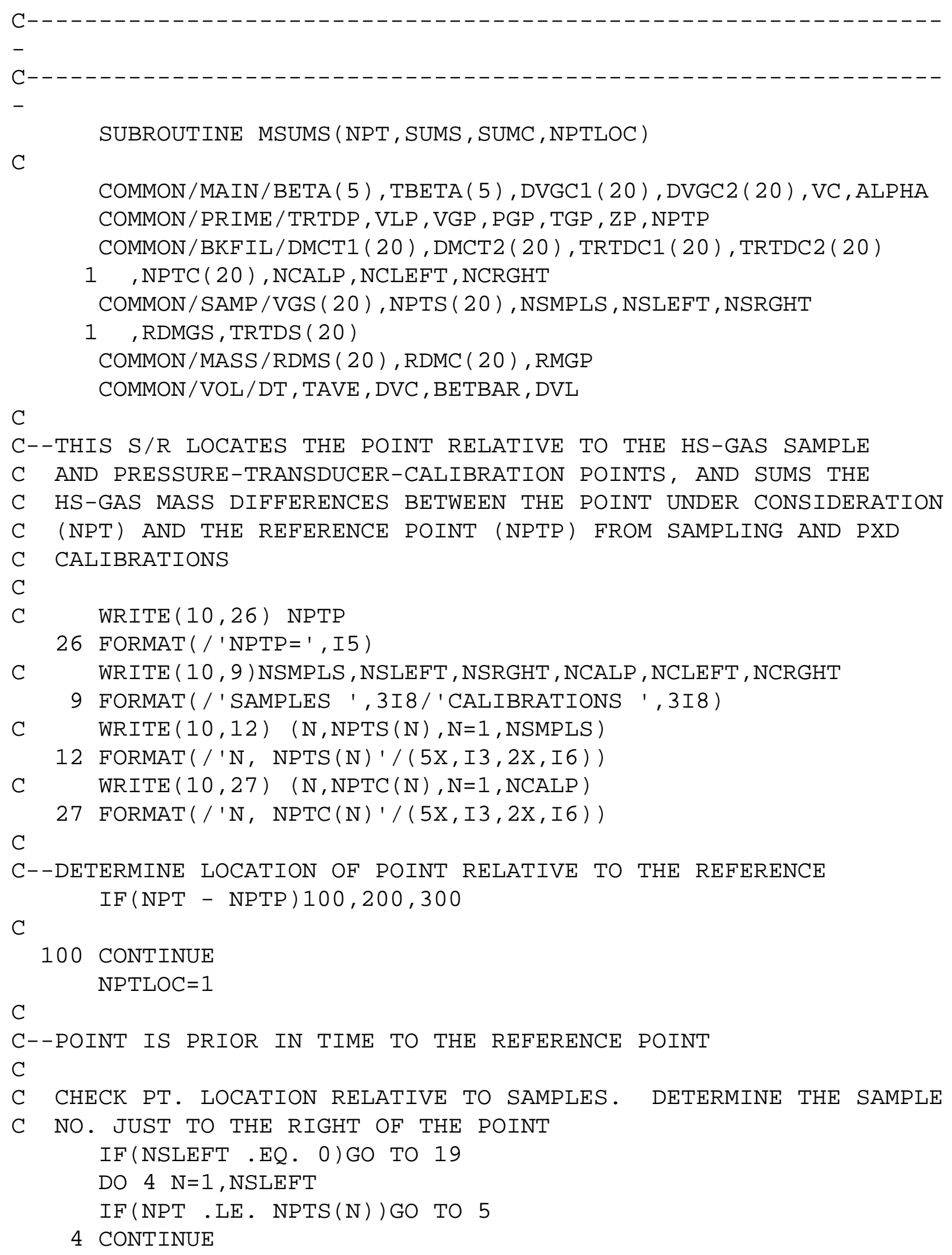


19 SUMS $=0$.

GO TO 15

C

5 SUMS $=0$.

DO $13 \mathrm{I}=\mathrm{N}, \mathrm{NSLEFT}$

SUMS $=$ SUMS+RDMS $(I)$

13 CONTINUE

C

15 CONTINUE

C

C--SIMILARLY, CHECK PT. LOCATION RELATIVE TO PXD CALIBRATIONS

C DETERMINE THE CALIBRATION NO. JUST TO THE RIGHT OF THE POINT IF (NCLEFT .EQ. $\odot)$ GO TO 14

DO $16 \mathrm{~N}=1$, NCLEFT

IF(NPT . LE. NPTC(N))GO TO 17

16 CONTINUE

14 SUMC $=0$.

RETURN

C

$17 \mathrm{SUMC}=0$.

DO $18 \mathrm{I}=\mathrm{N}, \mathrm{NCLEFT}$

SUMC $=S U M C+R D M C(I)$

18 CONTINUE

RETURN

C

C

$30 \odot$ CONTINUE

NPTLOC $=3$

C

C--POINT IS BEYOND THE REFERENCE POINT IN TIME

C

C--CHECK PT. LOCATION RELATIVE TO SAMPLES DETERMINE THE NO. OF

C THE LAST SAMPLE BEFORE THE POINT.

IF(NSRGHT .GT. NSMPLS)GO TO 7

C

DO $6 \mathrm{~N}=\mathrm{NSRGHT}$, NSMPLS

NBK=NSMPLS+NSRGHT $-\mathrm{N}$

IF(NPT .GT. NPTS(NBK))GO TO 8

6 CONTINUE

C

7 CONTINUE

SUMS $=0$.

GO TO 22

C

8 SUMS $=0$.

DO $20 \mathrm{I}=\mathrm{NSRGHT}$, NBK 


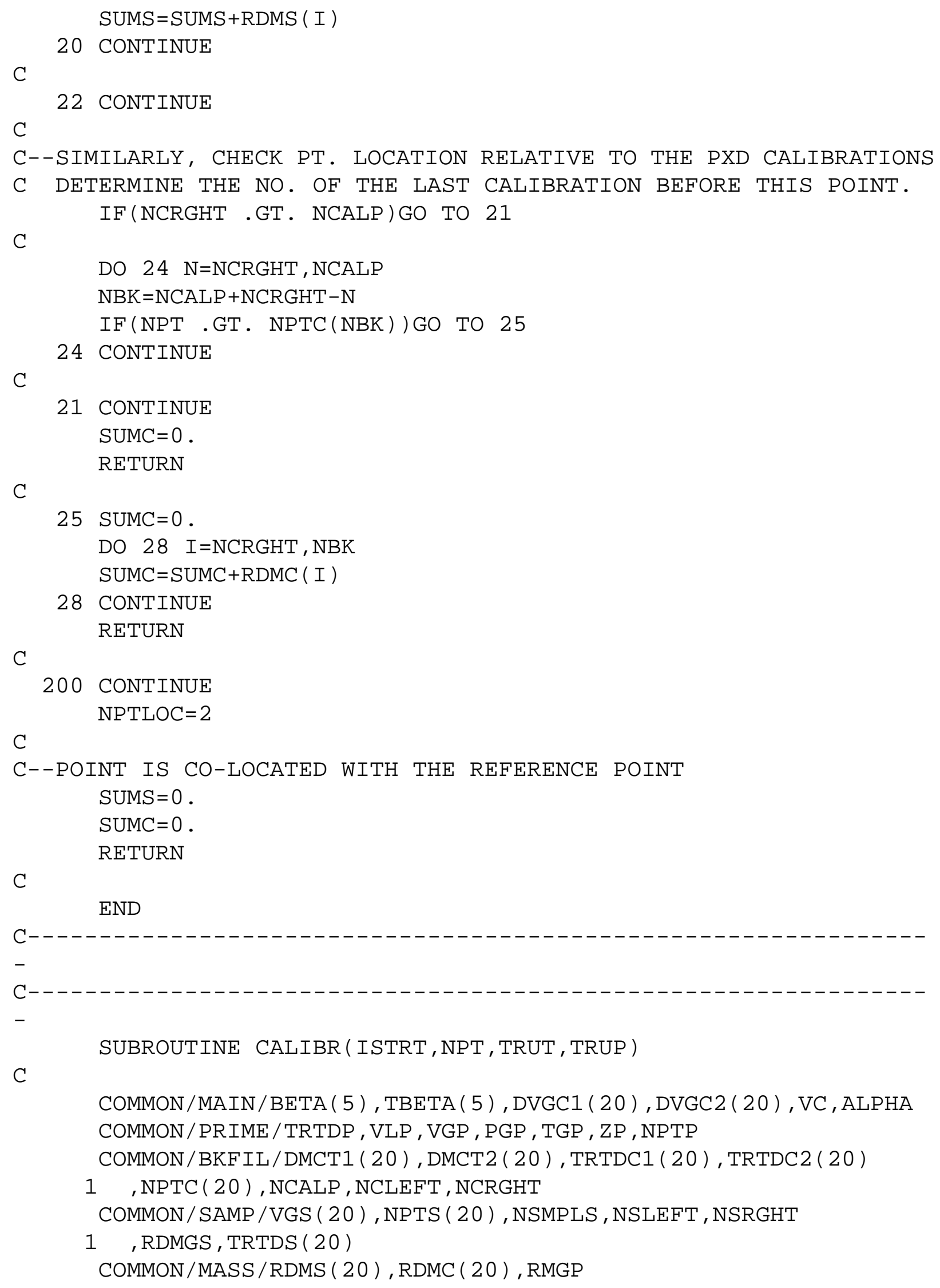




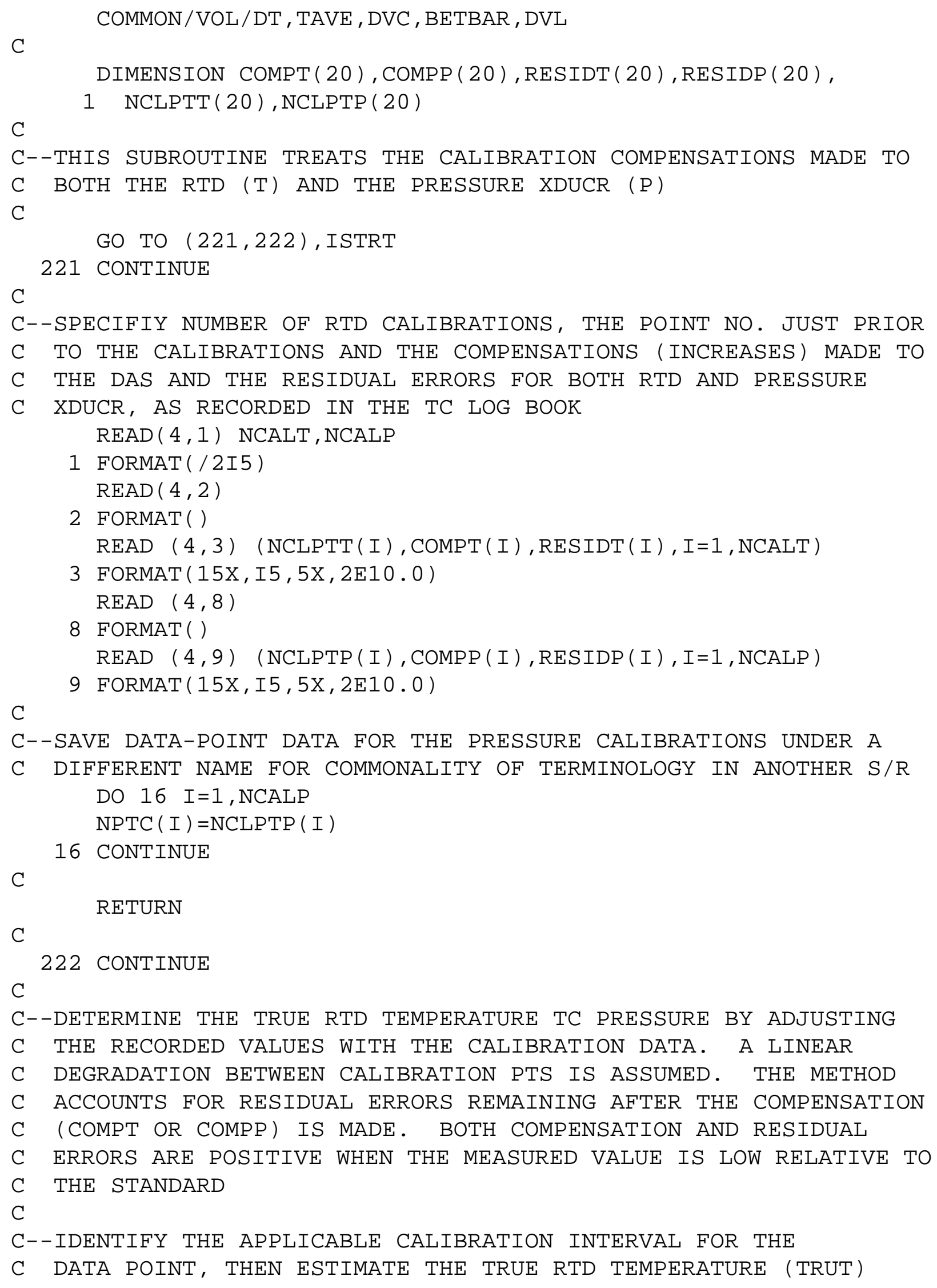




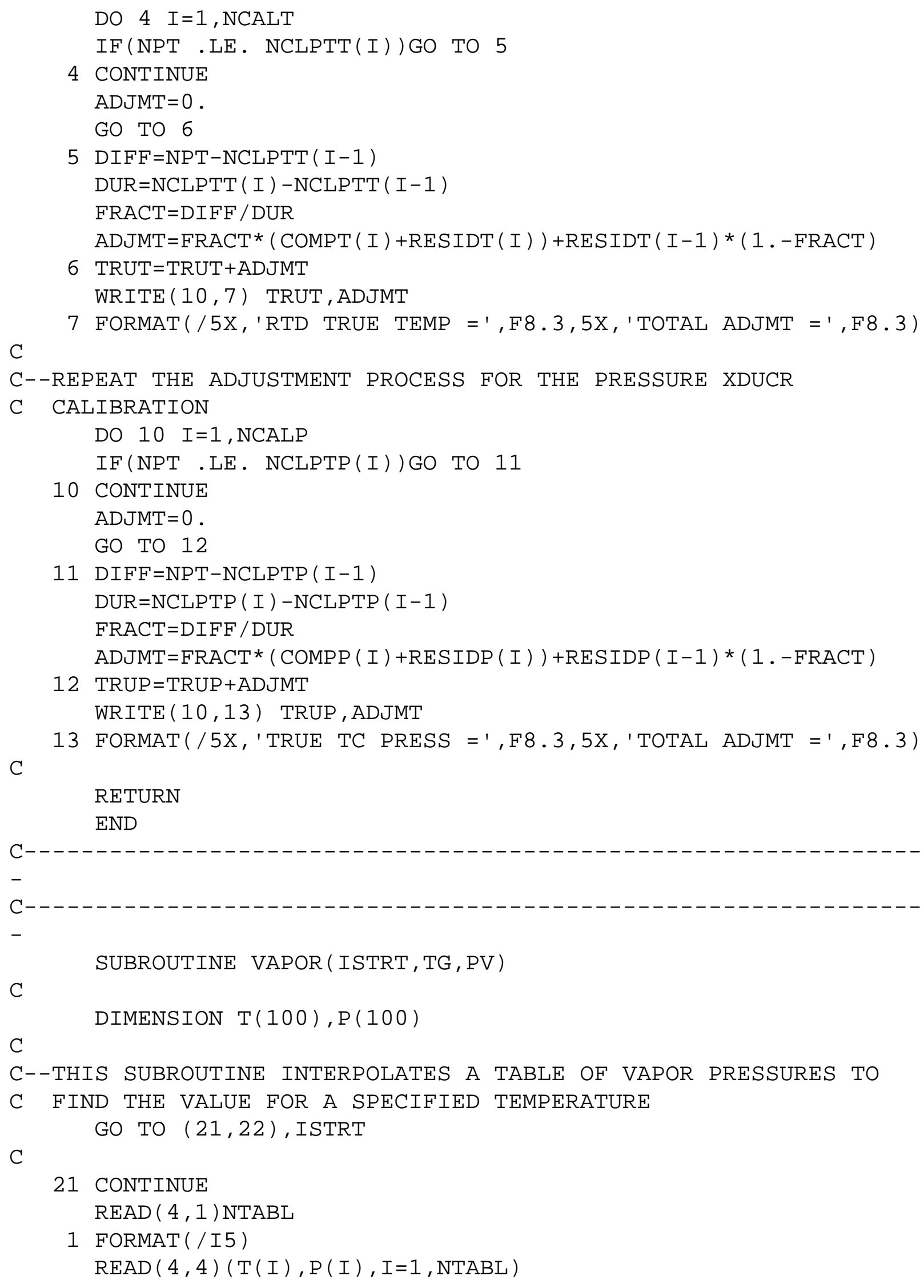




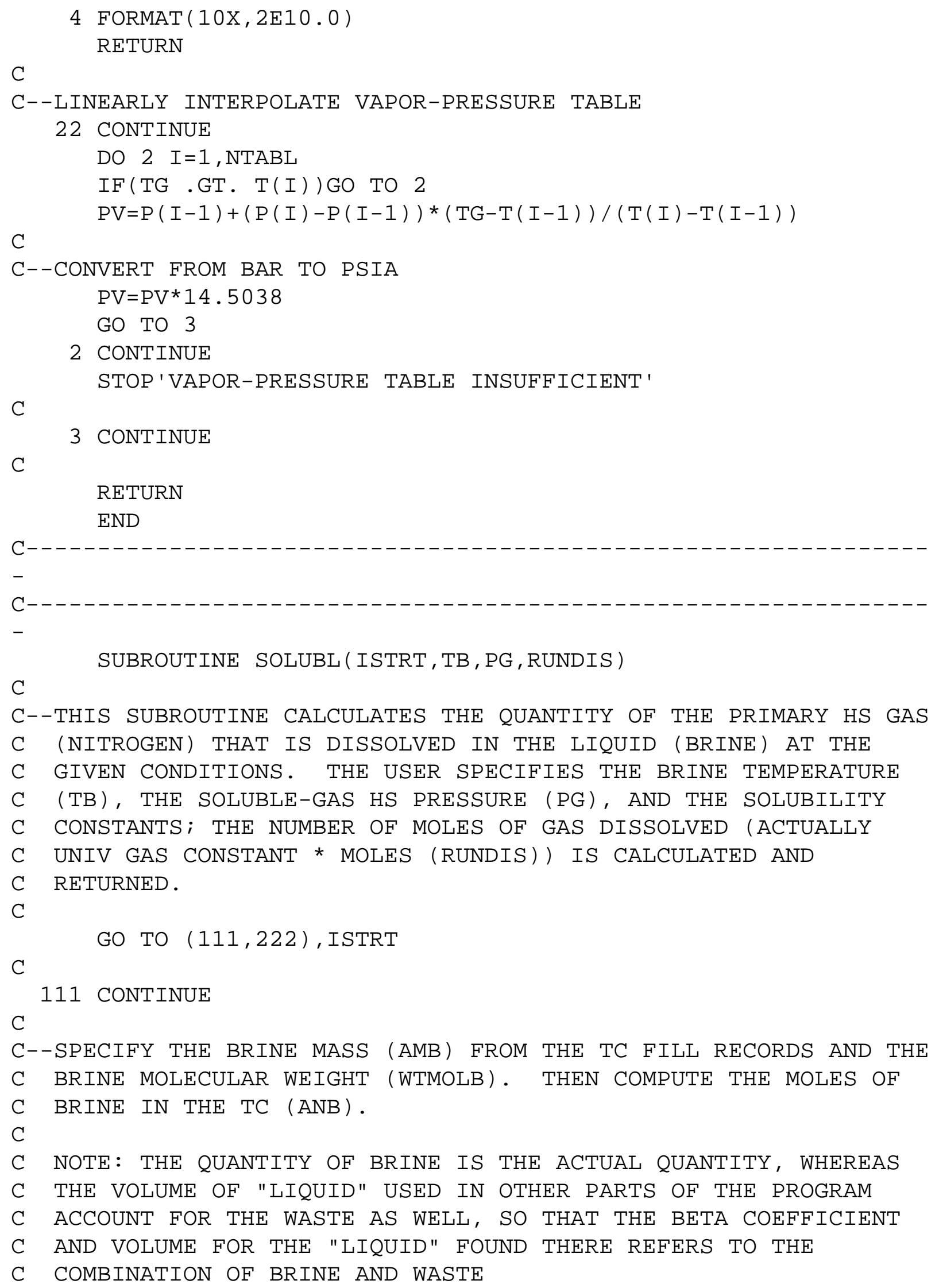


C $\operatorname{READ}(4,3)$ AMB, WTMOLB

3 FORMAT (/2E10.0) $A N B=A M B / W T M O L B$

C

C--SPECIFY THE UNIVERSAL GAS CONSTANT (RU) AND THE STANDARD

C ATMOSPHERIC PRESSURE (PATM) IN CONSISTENT UNITS - EG, WITH

C $[\mathrm{T}]=\mathrm{K},[\mathrm{P}]=\mathrm{PSIA},[\mathrm{VS}]=\mathrm{ML},[\mathrm{AMB}]=\mathrm{G}, \&[$ WTMOLB] $=\mathrm{G} / \mathrm{GMOLE}$ THEN

C PATM $=14.696$ PSIA \& RU $=1205.95$ PSIA*ML/GMOLE/K $\operatorname{READ}(4,4)$ RU, PATM

4 FORMAT $(/ 2 \mathrm{E} 10 . \odot)$

C

C--SPECIFY THE VOLUMETRIC FRACTION (VFRG), MOLECULAR WT (WTMOLG)

C AND THE ASSOCIATED SOLUBILITY PARAMETERS A, B, \& C PER THE

C RELATIONS CITED IN CRC HANDBOOK OF CHEMISTRY \& PHYSICS FOR THE

C SOLUBLE GAS [VFRG $=0$. GIVES THE INSOLUBLE-GAS RESULTS].

$\mathrm{C}$

C--NOTE: THE CURRENT FORMULATION DOES NOT ACCOUNT FOR CHANGES IN

C THE TOTAL MASS (HS + DISSOLVED) OF SOLUBLE GAS SPECIES WITHIN

C THE TEST CONTAINER THAT RESULTS FROM GAS GENERATION

$\mathrm{C}$

$\operatorname{READ}(4,1)$ VFRG, $A, B, C$

1 FORMAT $(/ 4 \mathrm{E} 10.0)$

C

WRITE $(10,2)$ AMB , ANB, WTMOLB, RU, PATM, VFRG, A, B, C

2 FORMAT( / 'SOLUBILITY PARAMETERS: ' /5X, 'AMB= ' , 4PE15.4/5X,

1 'ANB= ' , 3PE15.4/5X, 'WTMOLB=' , E12.4/5X, 'RU= ' , 4PE15.4/5X,

2 'PATM=' , F8.3/5X, 'VFRG=' , E12.4/5X, 'A, B, C=' , 3E15.5)

C

222 CONTINUE

$\mathrm{C}$

C--COMPUTE THE MOLE FRACTION OF THE GAS SPECIES DISSOLVED IN THE

C BRINE AT ONE ATMOSPHERE PARTIAL PRESSURE IN THE HS (X) FOR THE

C THE PRESENT CONDITIONS

TSTAR $=(T B+273.15) / 100$.

$A L N X=A+B / T S T A R+C * A L O G(T S T A R)$

$X=E X P(A L N X)$

C

C--USE HENRY'S LAW TO ACCOUNT FOR THE ACTUAL PARTIAL PRESSURE

C (PPRES) OF THE SOLUBLE GAS SPECIES WITHIN THE HS [THE PARTIAL

C PRESSURE OF A SPECIE EQUALS THE VOL FRACTION TIMES THE TOTAL

C PRESSURE OF ALL GASES WITHIN THE HS]

$P P R E S=V F R G * P G$

C

C DETERMINE THE MOLE FRACTION OF THE DISSOLVED GAS SPECIES IN

C SOLUTION AT THE ACTUAL GAS PRESSURE (SX) AND THE ACTUAL NUMBER 


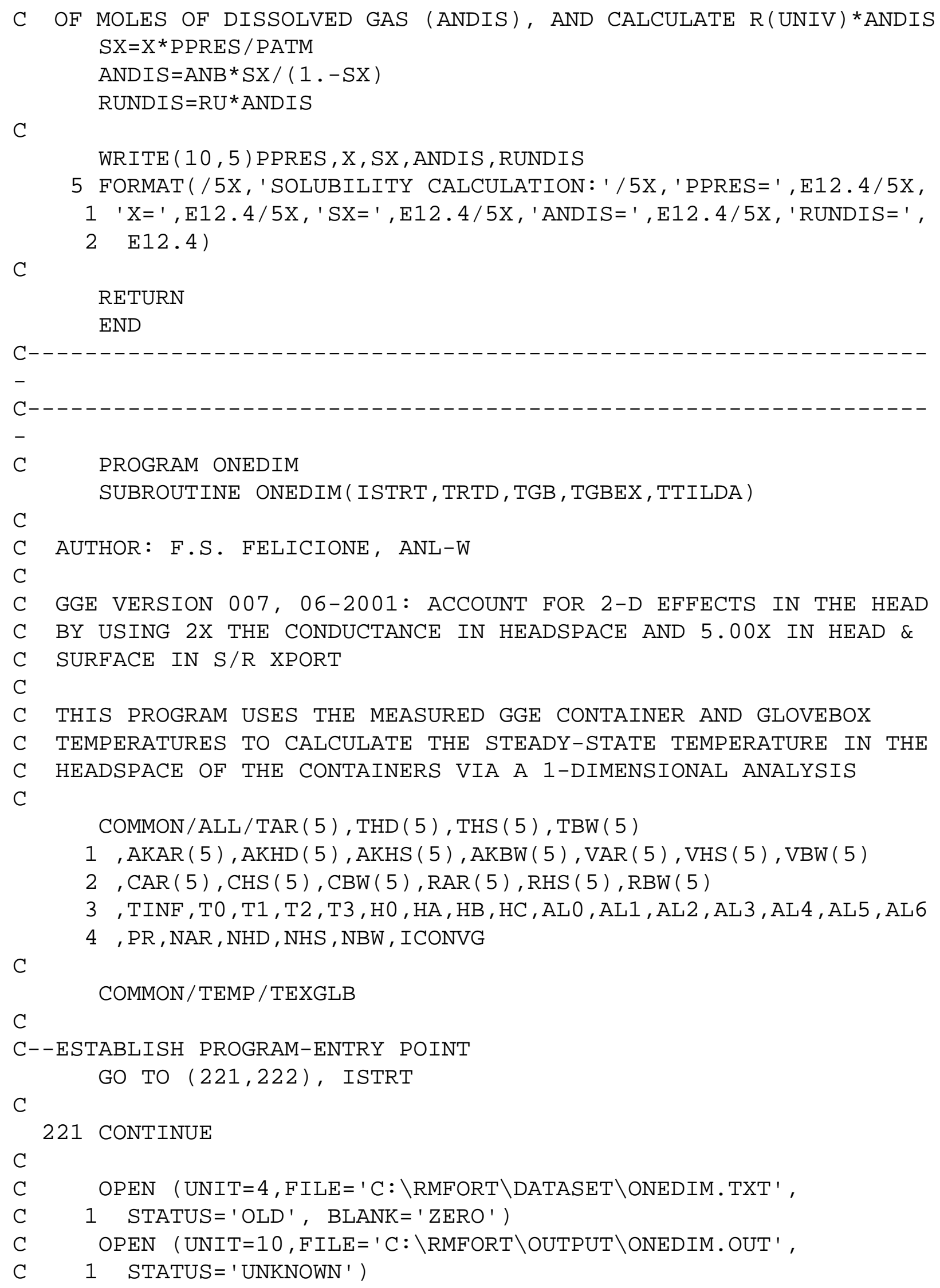




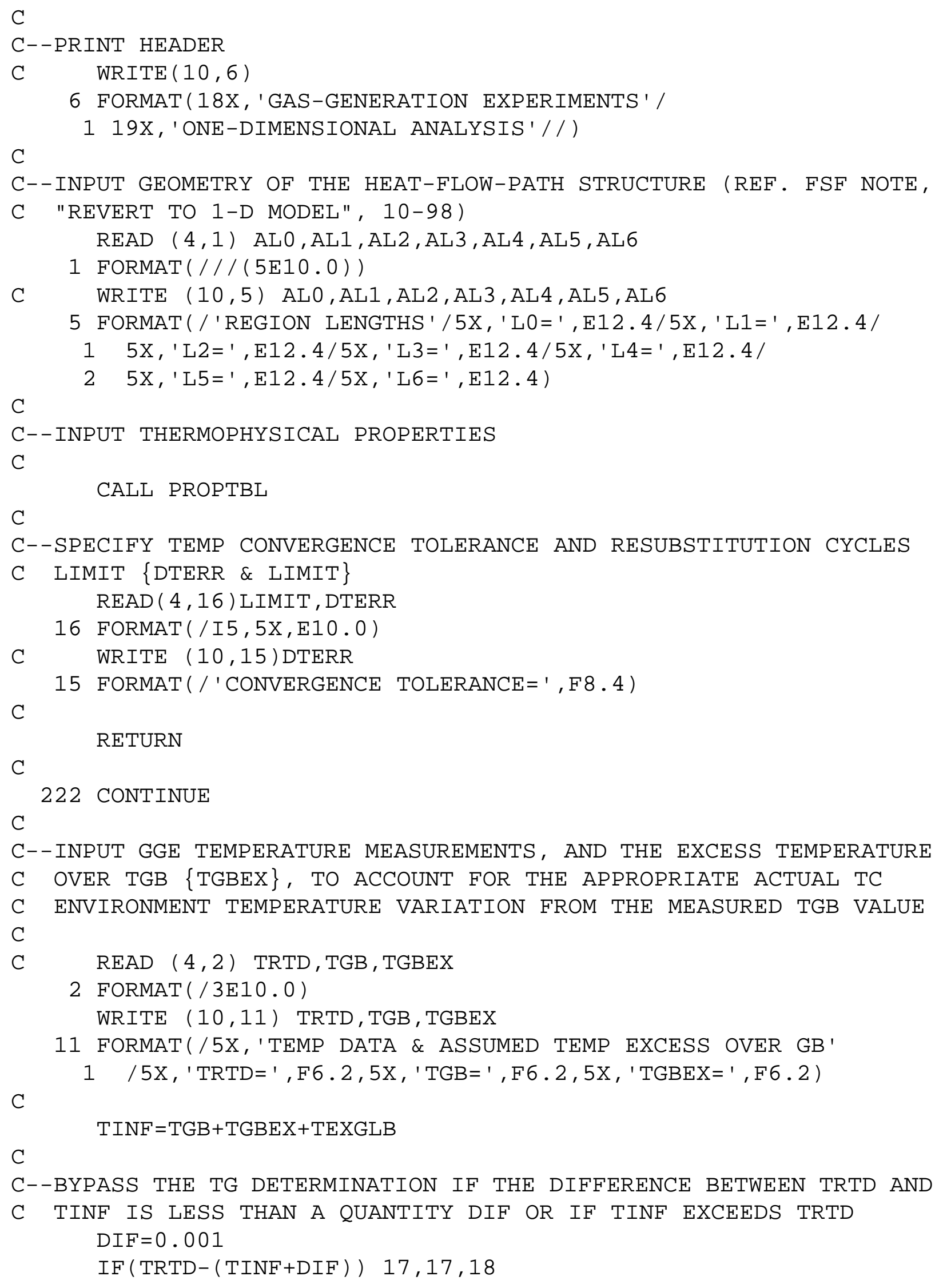




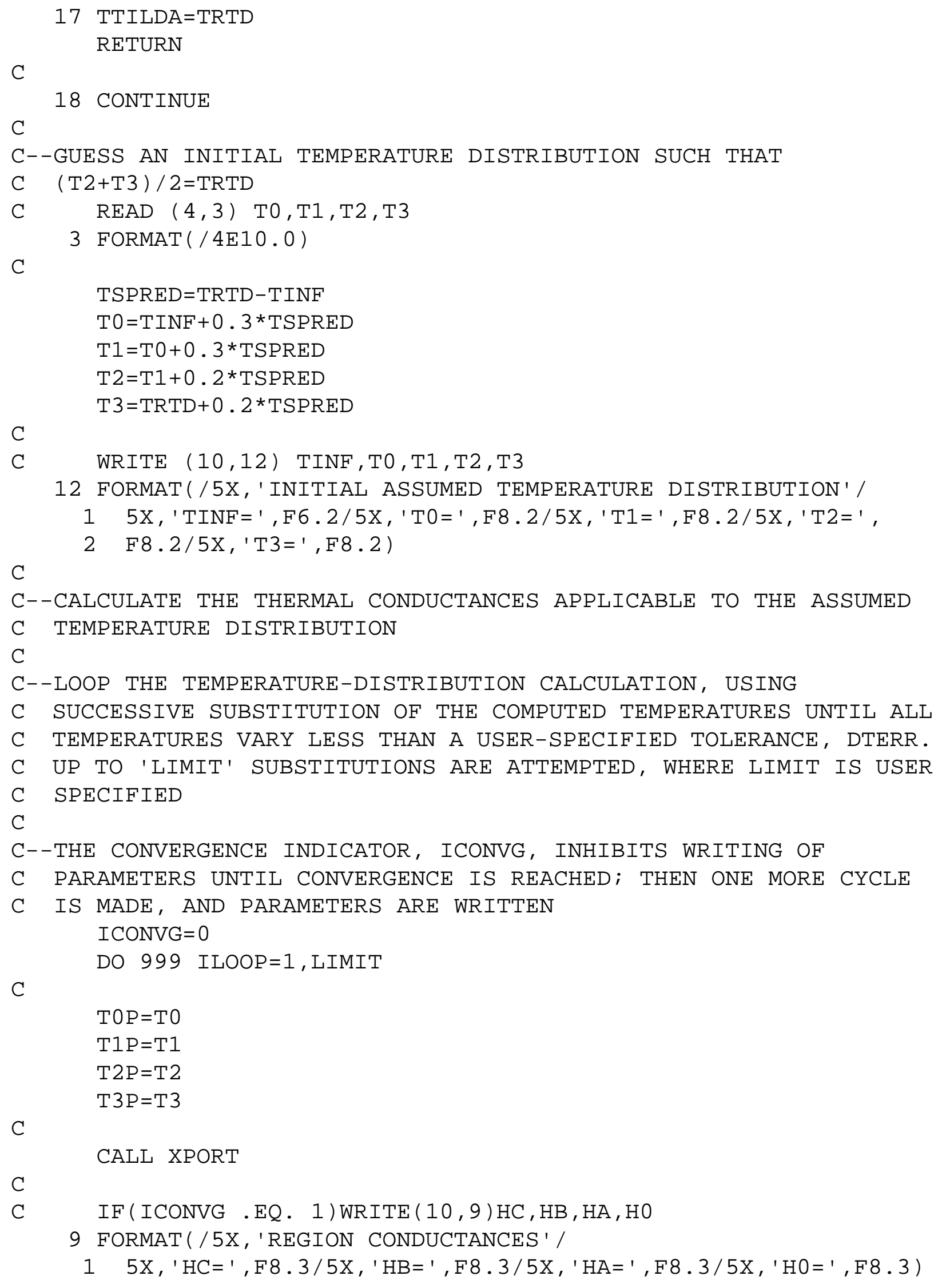




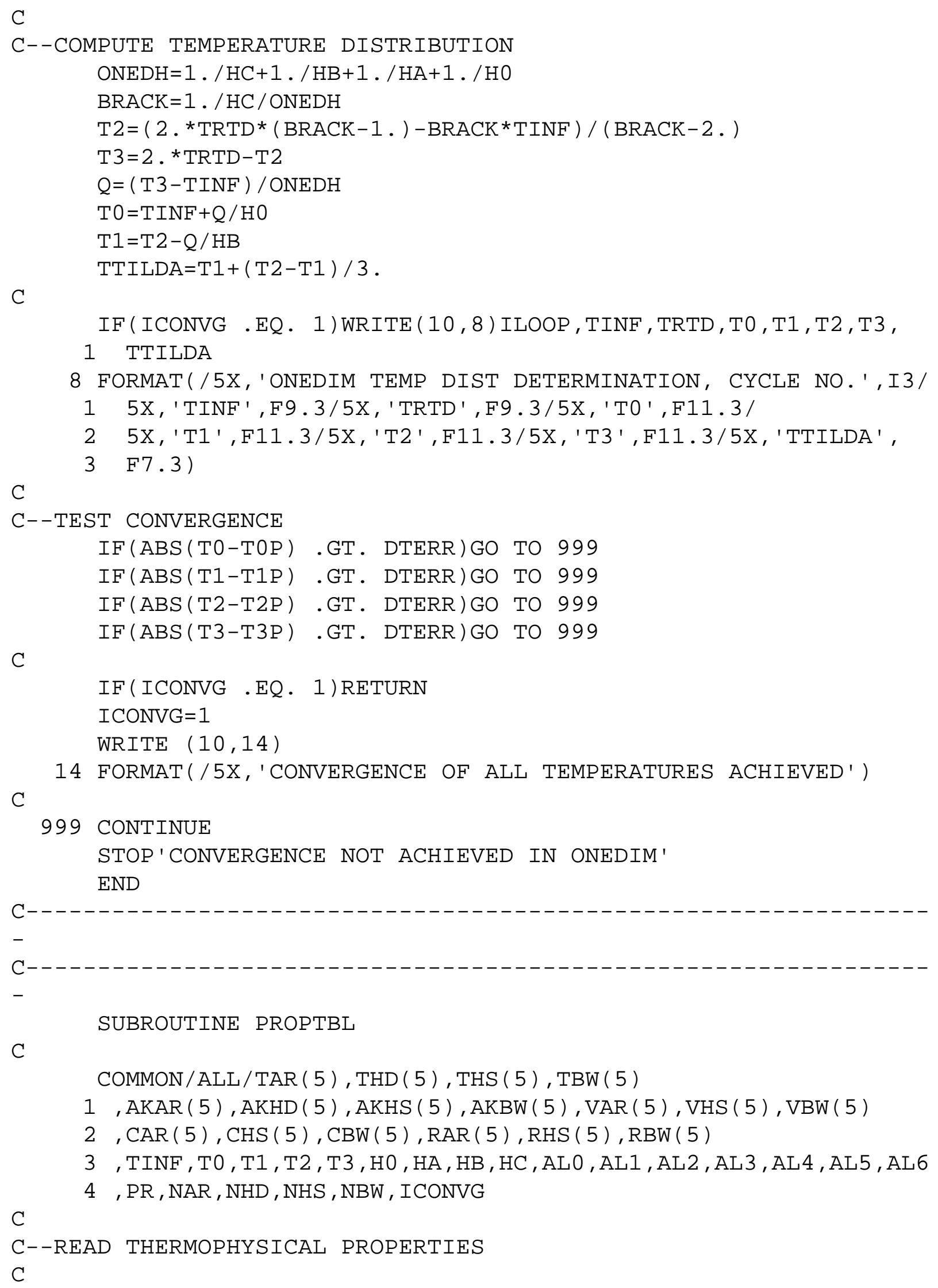




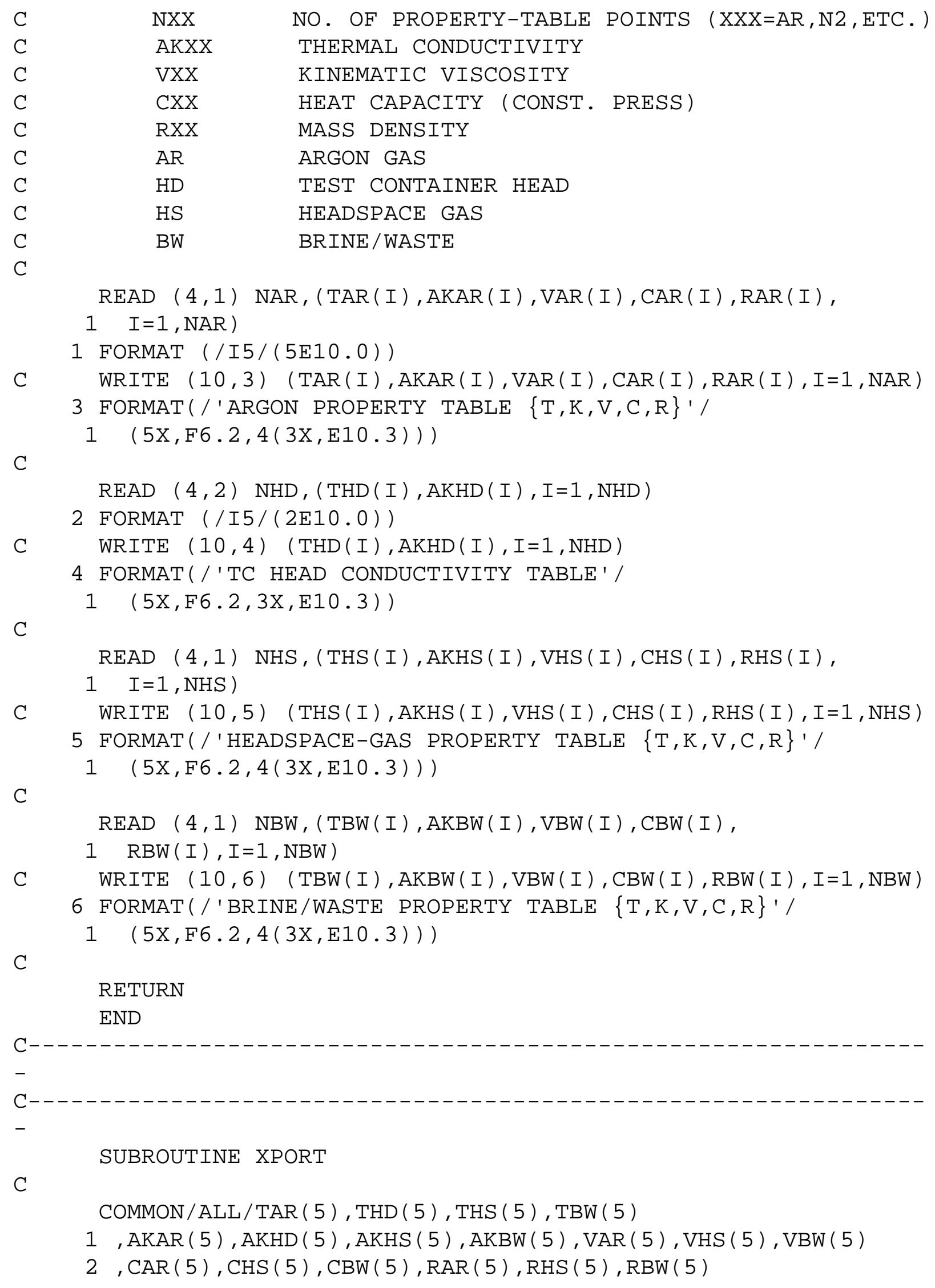

$\mathrm{C}$
-
$\mathrm{C}-$
-
$\mathrm{C}$

\section{SUBROUTINE XPORT}

COMMON/ALL/TAR( 5$), \operatorname{THD}(5), \operatorname{THS}(5), \operatorname{TBW}(5)$

$1, \operatorname{AKAR}(5), \operatorname{AKHD}(5), \operatorname{AKHS}(5), \operatorname{AKBW}(5), \operatorname{VAR}(5), \operatorname{VHS}(5), \operatorname{VBW}(5)$

$2, \operatorname{CAR}(5), \operatorname{CHS}(5), \operatorname{CBW}(5), \operatorname{RAR}(5), \operatorname{RHS}(5), \operatorname{RBW}(5)$ 


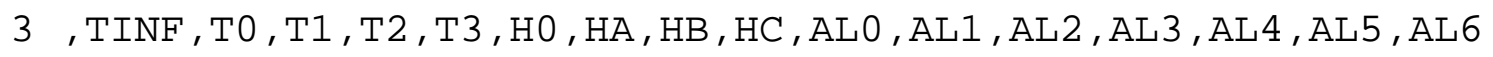

4 , PR, NAR, NHD, NHS, NBW, ICONVG

C

C TAVE AVERAGE TEMPERATURE

C DIFXX THERMAL DIFFUSIVITY

C BETAXX THERMAL EXPANSION COEFFICIENT

C RAXX RAYLEIGH NUMBER

C $\quad$ PRXX PRANDTL NUMBER

C RACXX CRITICAL RAYLEIGH NO.

C RACIXX SPECIFIC CRITICAL RAYLEIGH NO.

$\mathrm{C}$

C--DETERMINE CONDUCTANCE VALUES IN BRINE/WASTE (BW),

C HEADSPACE GAS (HS), TC HEAD (HD), AND TC SURFACE (AR)

C

C- - BRINE/WASTE

C

$\mathrm{TAVE}=(\mathrm{T} 2+\mathrm{T} 3) / 2$.

$\mathrm{DTBW}=\mathrm{T} 3-\mathrm{T} 2$

CALL TBLKUP ( $T A V E$, NBW, TBW, AKBW, VBW, CBW, RBW, AKVBW,

C

1 VVBW, CVBW, RVBW)

DIFBW $=$ AKVBW/RVBW/CVBW

C

C--LINEARIZE BRINE/WASTE BETA DATA

TBETAL $=20$.

$B E T A L=0.355 E-3$

$\mathrm{TBETAH}=40$.

$\mathrm{BETAH}=0.394 \mathrm{E}-3$

BETABW $=$ BETAL $+($ TAVE - TBETAL $) /($ TBETAH - TBETAL $) *(B E T A H-B E T A L)$

C

C IF( ICONVG .EQ. 1)WRITE $(10,23)$ TAVE, AKVBW, VVBW, CVBW, RVBW,

C 1 DIFBW, BETABW

23 FORMAT(/5X, 'BRINE/WASTE PROP $\{\mathrm{K}, \mathrm{V}, \mathrm{C}, \mathrm{R}, \mathrm{D}, \mathrm{BETA}\}$ TAVE=', $\mathrm{F} 6.2$

$1 /(2 \mathrm{X}, 4(3 \mathrm{X}, \mathrm{E} 10.4)))$

C

CALL RAYLEE (BETABW, AL6, VVBW, DIFBW, DTBW, RABW, PRBW)

IF ( ICONVG . EQ . 1)WRITE $(10,21)$ RABW, PRBW

C

$21 \operatorname{FORMAT}(/ 5 \mathrm{X}$, 'RA=' , E10.3, 5X, 'PR=' , E10.3)

RACIBW $=19494$.

RACBW=RACIBW

C

CALL NUSSLT (RABW, PRBW, RACBW, AL6, AKVBW, HC)

C

C--HEADSPACE GAS

C 


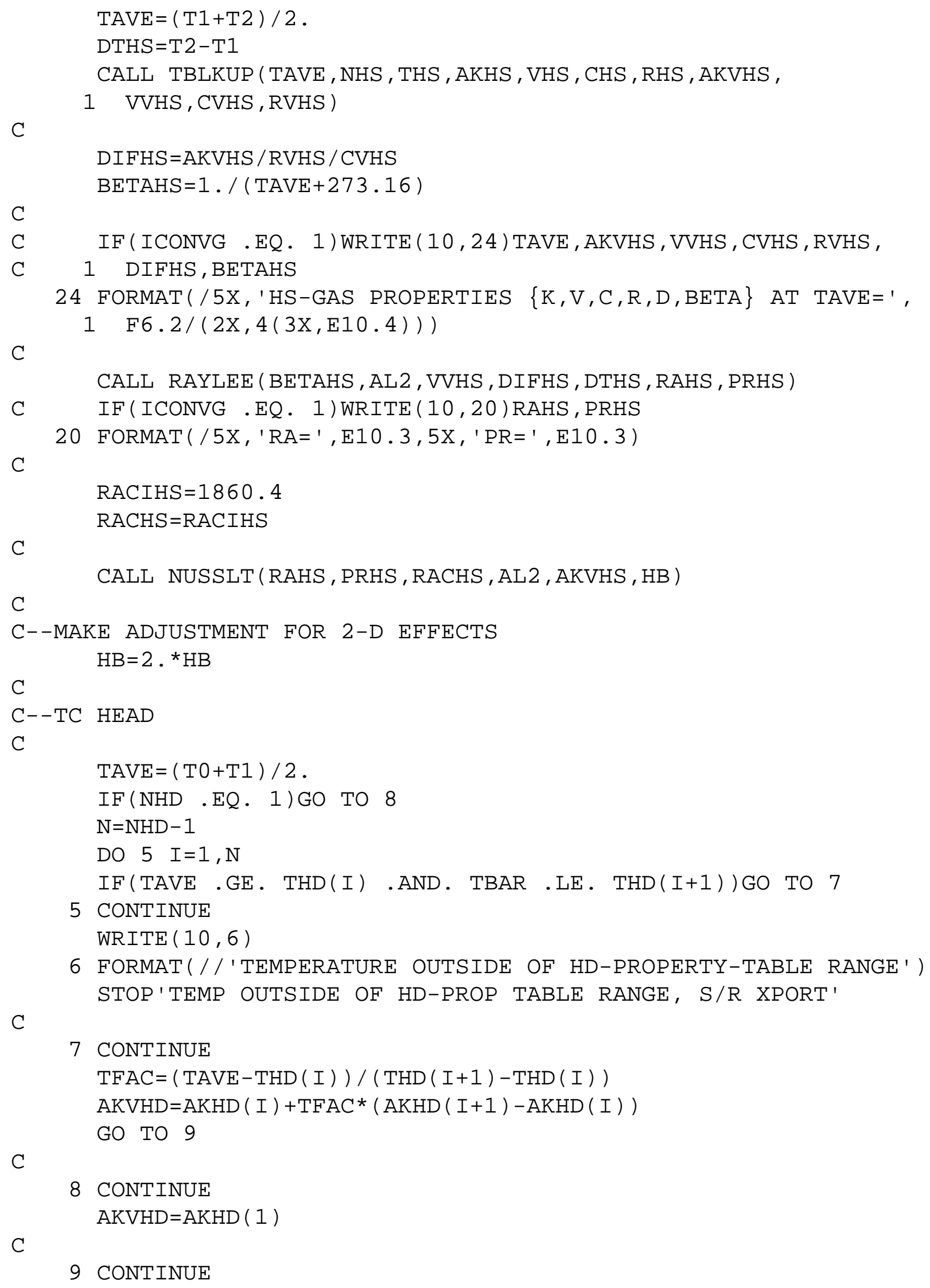




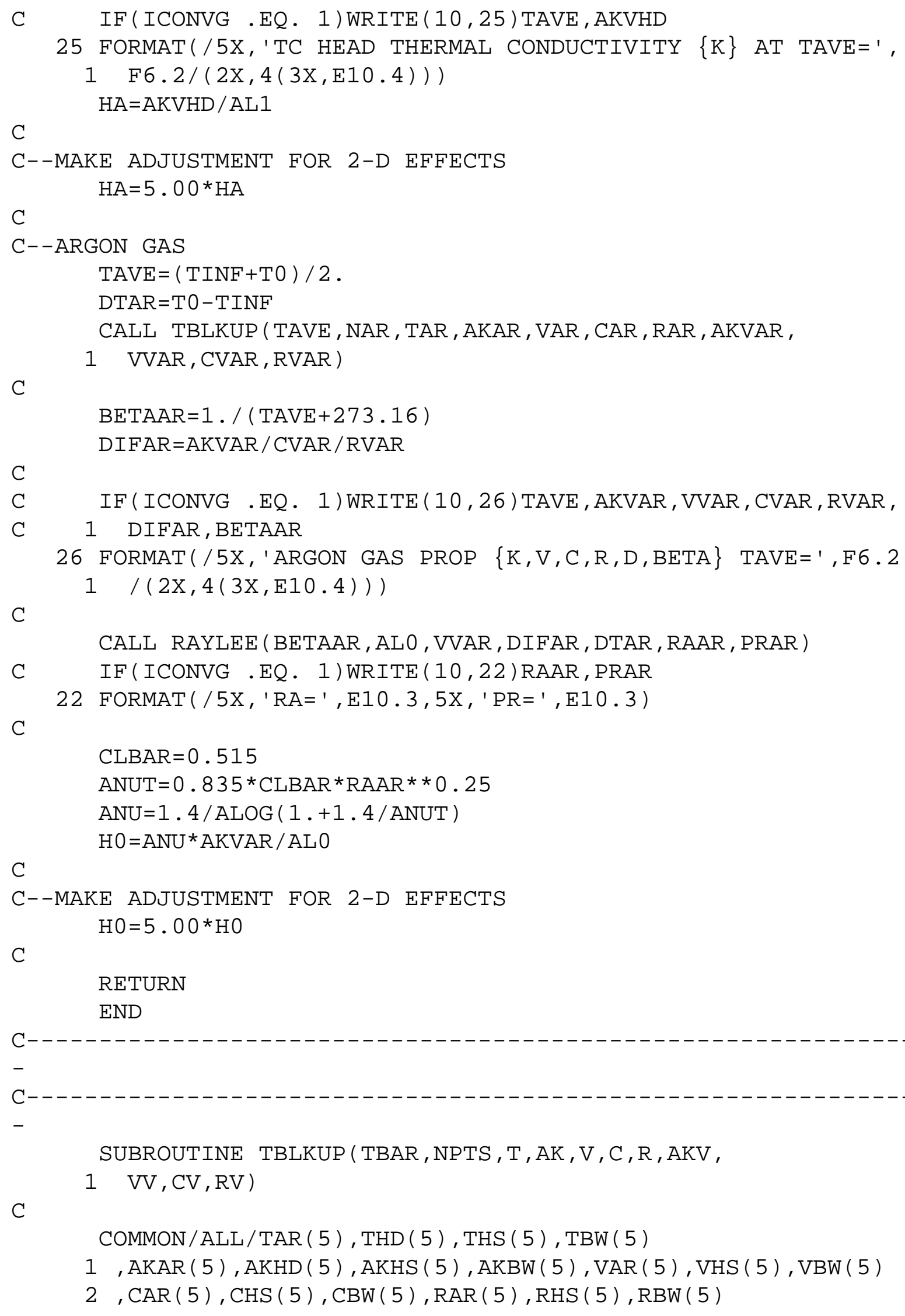




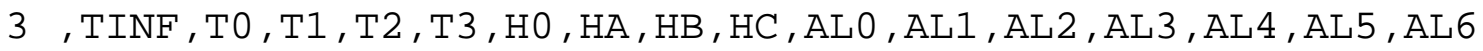

4 ,PR, NAR, NHD, NHS, NBW, ICONVG

C

DIMENSION $\mathrm{T}(1), \mathrm{AK}(1), \mathrm{V}(1), \mathrm{C}(1), \mathrm{R}(1)$

C

IF(NPTS .EQ. 1)GO TO 5

C

$\mathrm{N}=$ NPTS -1

D0 $2 \mathrm{I}=1, \mathrm{~N}$

IF(TBAR . GE. $T(I)$. AND. TBAR . LE. $T(I+1)) G O$ TO 3

2 CONTINUE

$\operatorname{WRITE}(10,4)$

4 FORMAT(//'TEMPERATURE OUTSIDE OF PROPERTY TABLE RANGE') $\operatorname{WRITE}(9,6)$ TBAR

6 FORMAT ( /'TBAR $=$ ', E12.4)

STOP'TEMP OUT OF PROP TABLE RANGE, S/R TBLKUP'

C

3 CONTINUE

TFAC $=(\operatorname{TBAR}-\mathrm{T}(\mathrm{I})) /(\mathrm{T}(\mathrm{I}+1)-\mathrm{T}(\mathrm{I}))$

$A K V=A K(I)+T F A C^{*}(A K(I+1)-A K(I))$

$V V=V(I)+T F A C^{*}(V(I+1)-V(I))$

$C V=C(I)+T F A C^{*}(C(I+1)-C(I))$

$R V=R(I)+T F A C *(R(I+1)-R(I))$

RETURN

C

5 CONTINUE

$A K V=A K(1)$

$\mathrm{VV}=\mathrm{V}(1)$

$\mathrm{CV}=\mathrm{C}(1)$

$\mathrm{RV}=\mathrm{R}(1)$

C

RETURN

END

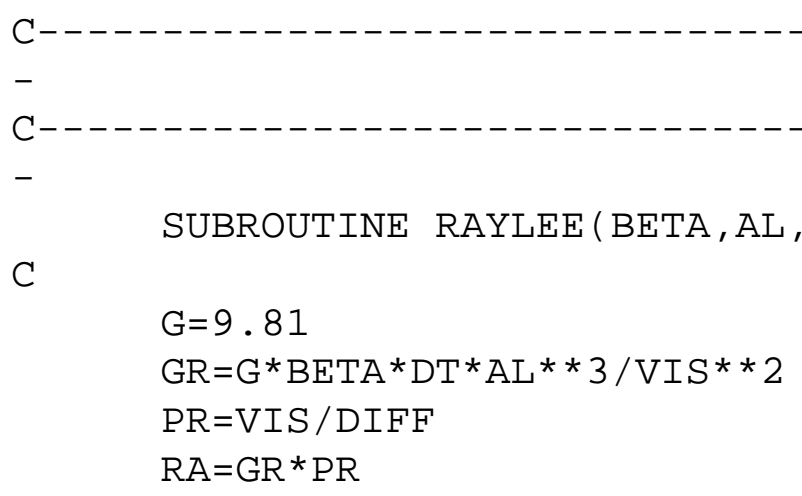

C

RETURN

END 


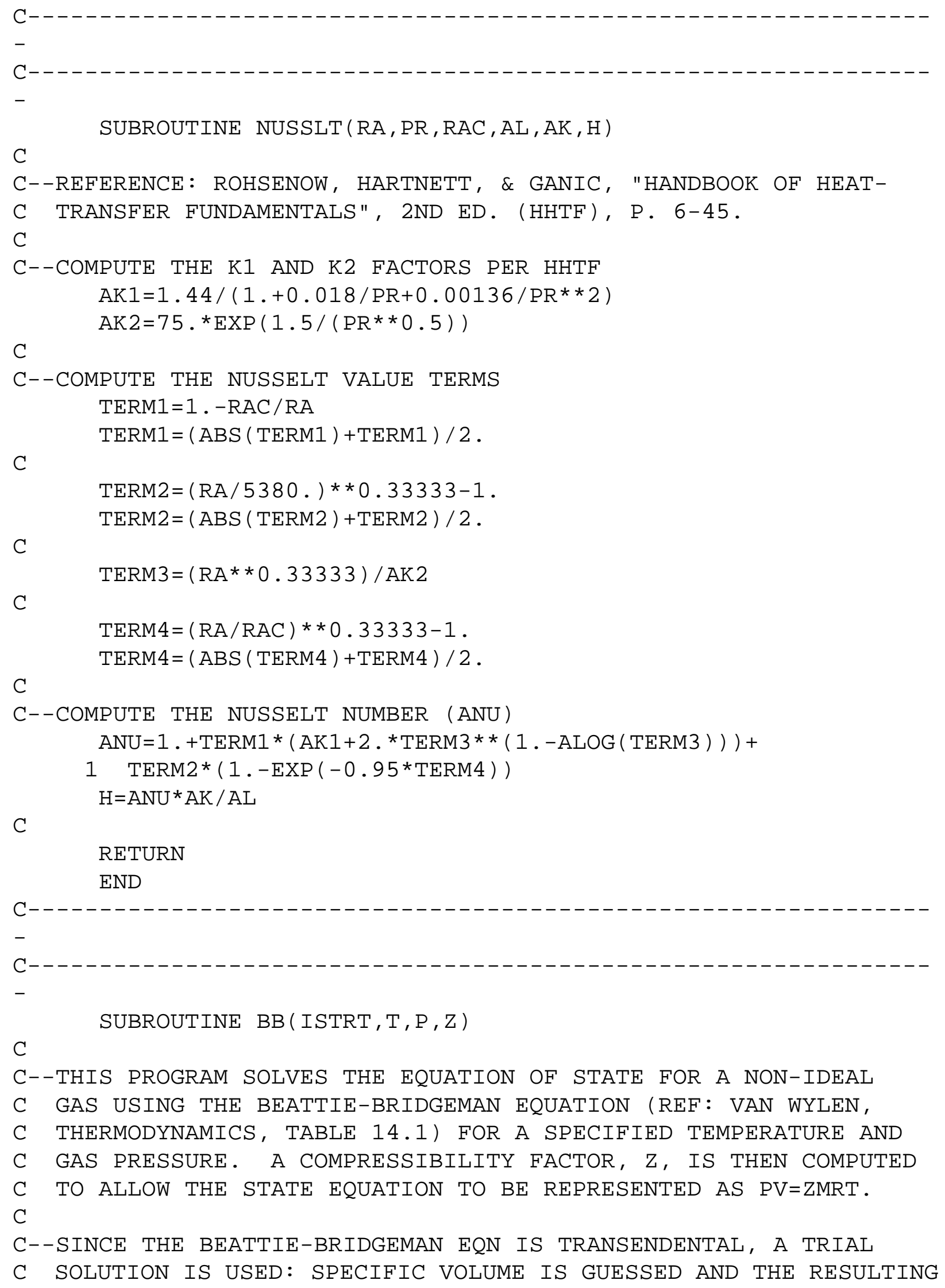




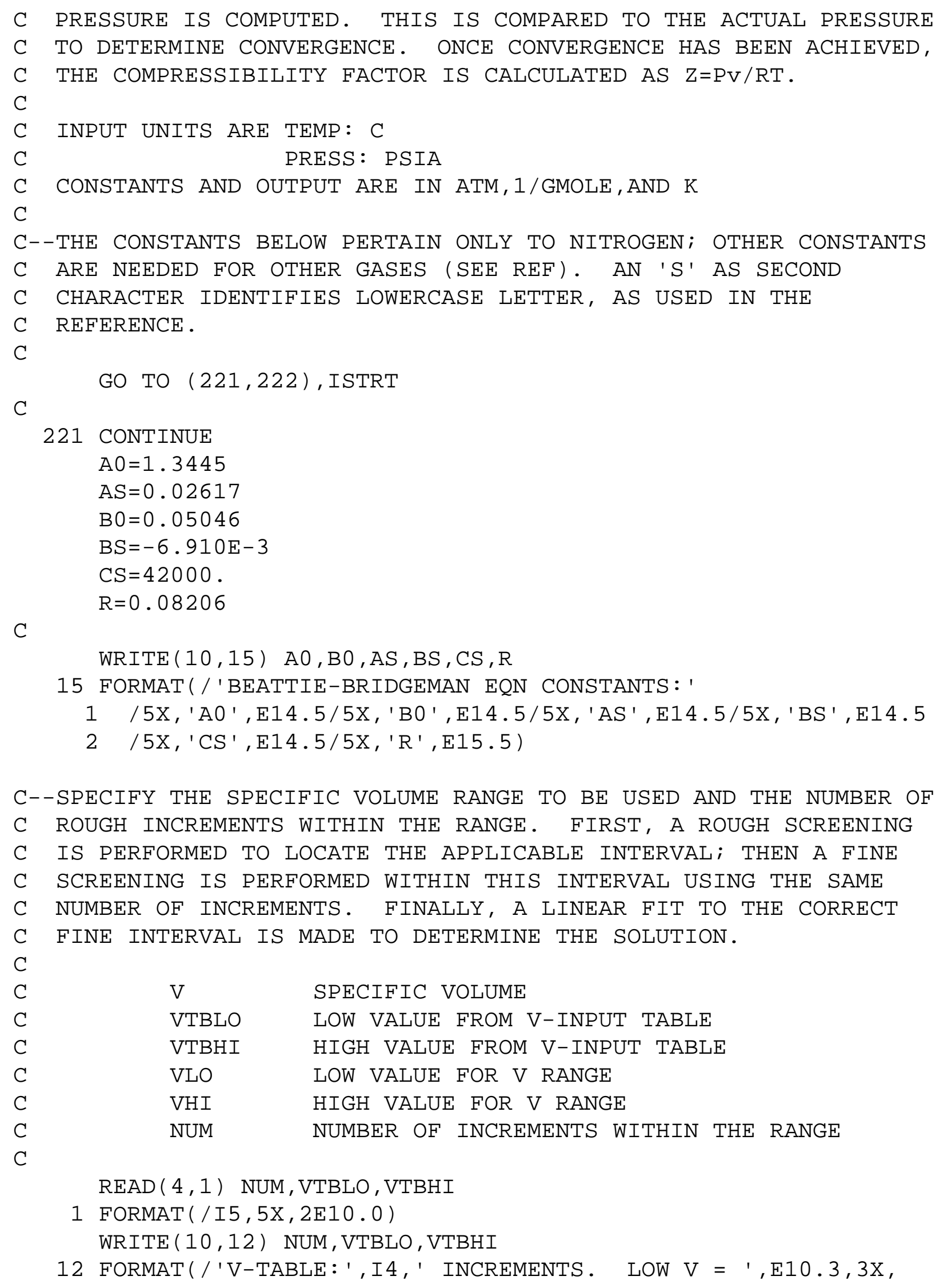


C

1 'HI V = ',E10.3)

RETURN

C

C--THE CHARACTERISTIC OF THE B-B EQUATION IS THAT A SPECIFIC

C VOLUME GUESS THAT IS TOO LOW WILL YIELD A CALULATED

C PRESSURE THAT EXCEEDS THE ACTUAL PRESSURE; CONVERSELY, IF THE

C SPECIFIC VOLUME USED IS TOO HIGH, THE CALCULATED PRESSURE IS

C LESS THAN THE ACTUAL PRESSURE. BUT THIS TREND SHOULD BE HAND

C VERIFIED FOR OTHER GASES BEFORE USING THIS ROUTINE.

$\mathrm{C}$

C--CALCULATE THE IMPLIED PRESSURE. THE FIRST TIME THROUGH IS A

C ROUGHING CALCULATION TO LOCATE THE PROPER INTERVAL; THE SECOND

C TIME THROUGH IS A FINE CALCULATION.

C

222 CONTINUE

$\operatorname{WRITE}(10,16) \mathrm{T}, \mathrm{P}$

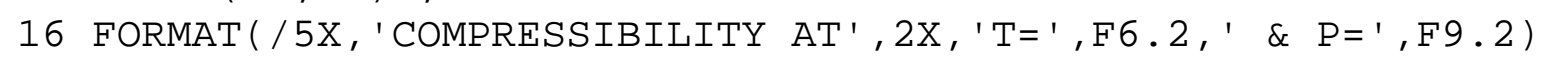

C

C WRITE $(10,3)$

3 FORMAT(/10X, 'V', 15X, 'PCALC', 10X, 'RATIO' )

$\mathrm{C}$

C-- CONVERT $[\mathrm{T}]=\mathrm{C}$ TO $[\mathrm{TK}]=\mathrm{K}$, AND $[\mathrm{P}]=\mathrm{PSIA}$ TO $[\mathrm{PA}]=\mathrm{ATM}$

$\mathrm{TK}=273.15+\mathrm{T}$

$\mathrm{PA}=\mathrm{P} / 14.696$

C

$\mathrm{DV}=(\mathrm{VTBHI}-\mathrm{VTBLO}) / \mathrm{NUM}$

$\mathrm{V}=\mathrm{VTBLO}$

NUMP $1=N U M+1$

C

D0 $2 I=1, N U M P 1$

$\mathrm{V} 1=\mathrm{V}$

$\mathrm{IF}(\mathrm{I}, \mathrm{GT}, 1) \mathrm{V}=\mathrm{V}+\mathrm{DV}$

$A=A \odot *(1 .-A S / V)$

$\mathrm{B}=\mathrm{BO} *(1,-\mathrm{BS} / \mathrm{V})$

$\mathrm{E}=\mathrm{CS} / \mathrm{V} / \mathrm{TK}^{*} * 3$

$P C A L C=\left(R^{*} T K^{*}(1,-E) *(V+B)-A\right) / V * * 2$

RATIO $=\mathrm{PCALC} / \mathrm{PA}$

C $\operatorname{WRITE}(10,5) \mathrm{V}, \operatorname{PCALC}, \operatorname{RATIO}$

5 FORMAT $(4 X, 3 E 15.7)$

C WRITE $(10,13) \mathrm{A}, \mathrm{B}, \mathrm{E}$

13 FORMAT(10X, 'A, B, E: ' , 5X, E13. 7, 2E15.7)

C

C--DETERMINE IF THE SOLUTION (RATIO = 1) LIES BETWEEN THIS V

C VALUE AND THE PREVIOUS VALUE: RATIO $>1$ MEANS THE CORRECT

C INTERVAL NOT YET REACHED; THE FIRST RATIO < 1 MEANS THAT THE 


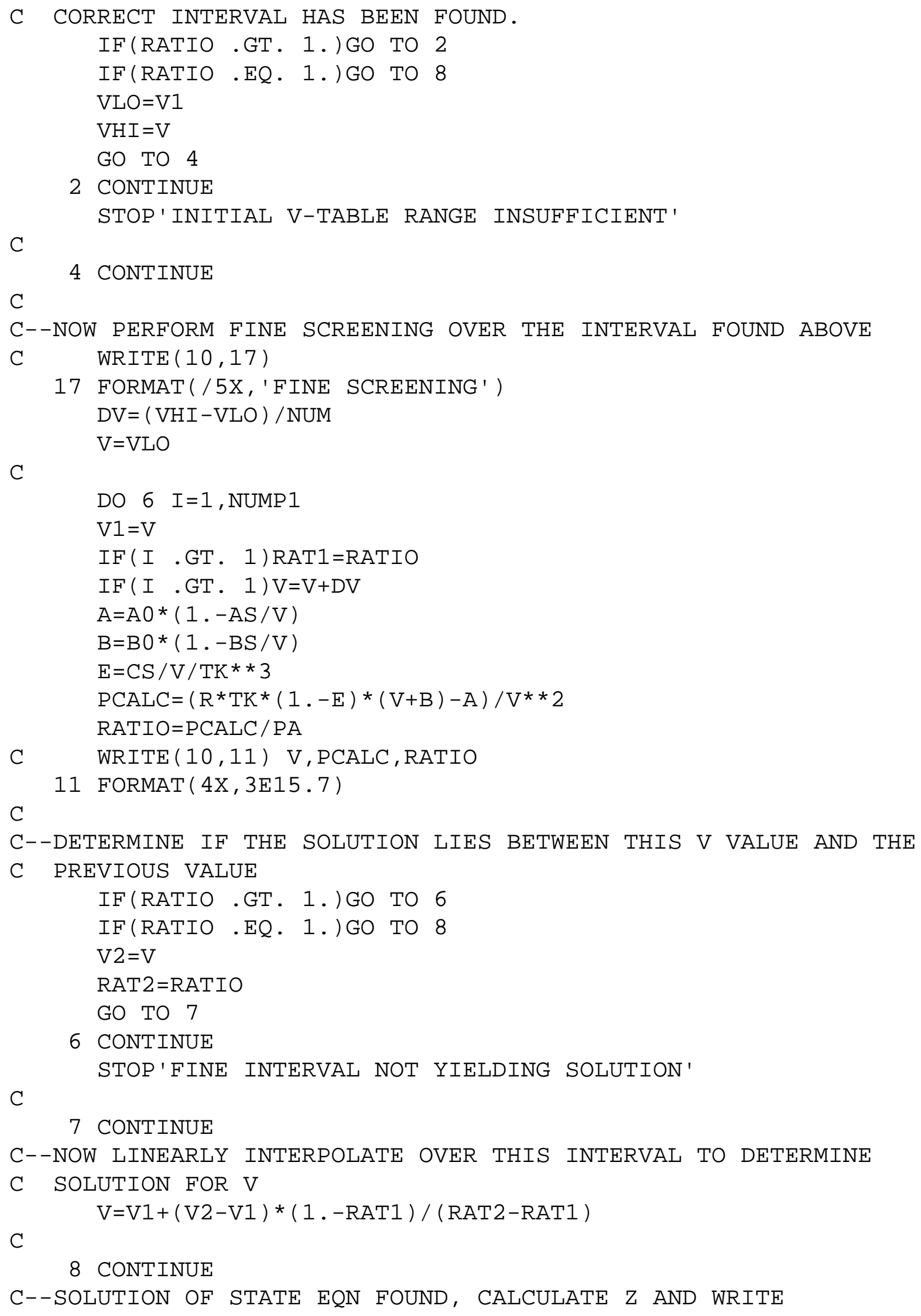




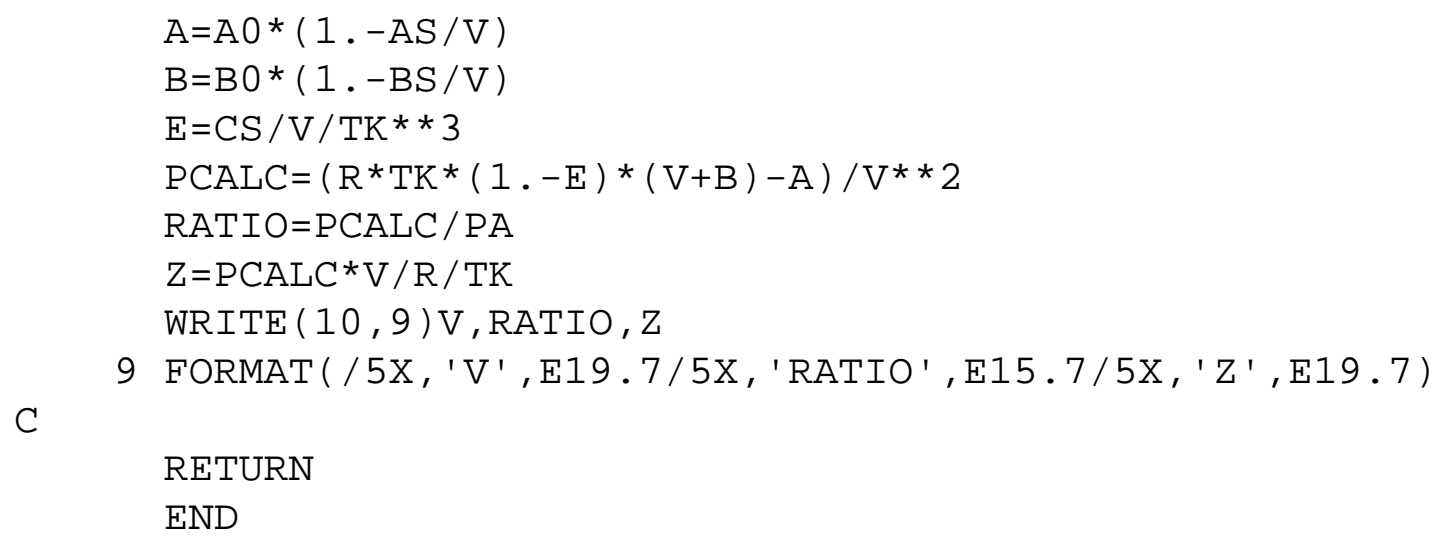

